\title{
Untersuchung von Feldumkehrungen an einem numerischen Modell des Geodynamos
}

\author{
Dissertation \\ zur Erlangung des Doktorgrades \\ der mathematisch-naturwissenschaftlichen Fakultäten \\ der Georg-August-Universität zu Göttingen
}

vorgelegt von

Carsten Kutzner

aus Göttingen

Göttingen 2003 
D 7

Referent: Prof. Dr. U. R. Christensen

Korreferent: Dr. D. Schmitt

Tag der mündlichen Prüfung: 27. 03. 2003 


\section{Inhaltsverzeichnis}

1 Einleitung 5

2 Das numerische Modell 11

2.1 Magneto-hydrodvnamische Gleichungen . . . . . . . . . . . . . . 12

2.2 Antrieb der Konvektion . . . . . . . . . . . . . . . . . . . . . . . 13

2.3 Numerische Methode . . . . . . . . . . . . . . . . . . . . . . . . 15

2.4 Rand- und Anfangsbedingungen $\ldots \ldots \ldots \ldots \ldots$

2.5 Numerische Auflösung . . . . . . . . . . . . . . . . . . . . 17

3 Der Parameterbereich für umkehrende Dvnamos 19

3.1 Umkehrende Dvnamos bei hoher Ekmanzahl und großem Radienverhältnis 21

3.2 Parameterabhängigkeit der Dynamoregimes . . . . . . . . . . . . . 27

3.3 Einfluß der Antriebsart . . . . . . . . . . . . . . . . . . . . . . . . . . 31

3.4 Polumkehrungen . . . . . . . . . . . . . . . . . . . . . . . . 35

3.5 Vergleich mit der Erde $\ldots \ldots \ldots \ldots \ldots \ldots$

4 Der Einfluß des Mantels auf den umpolenden Geodvnamo 43

4.1 Zusammenfassung thermischer und chemischer Konvektion . . . . . . . . . 47

4.2 Vorgehensweise . . . . . . . . . . . . . . . . . . . 48

4.3 Voruntersuchung bei großer Ekmanzahl . . . . . . . . . . . . . . . 51

4.3.1 Ein periodisch umkehrender Dvnamo . . . . . . . . . . . . . 51

4.3.2 Ortsabhängiger Wärmefluß bei moderaten Parametern . . . . . . 56

4.4 Einfluß eines longitudinal variierenden KMG-Wärmeflusses auf die VGP-

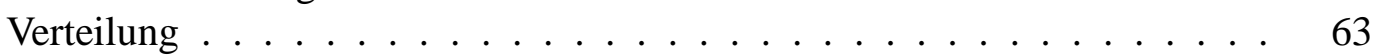

4.4.1 Verteilung und Anzahl der Sites . . . . . . . . . . . . . . 66

4.4 .2 Länge der Zeitreihe . . . . . . . . . . . . . . . . . . . . 67

4.4.3 Sites über hohem und niedrigem Wärmefluß . . . . . . . . . . 70

4.4 .4 Mittlere Verteilung der virtuellen Pole . . . . . . . . . . . . . . 71

4.4 .5 Verschiedene Amplituden der Modulation . . . . . . . . . . . . 74

4.4 .6 Tomographische Modulation . . . . . . . . . . . . . . . . . . 75

$4.4 .7 \quad$ VGP-Verteilungen einzelner Umkehrungen . . . . . . . . . . . . 79

4.5 Einfluß des KMG-Wärmeflußmusters auf die Umkehrfrequenz . . . . . . . 89

$\begin{array}{lll}5 \text { Zusammenfassung } & 95\end{array}$ 


\section{Einleitung}

Einer der interessantesten Aspekte des Erdmagnetfeldes ist seine auf geologischen Zeitskalen wechselnde Polarität. Die heutige, ,normale“ Polarität, bei der der magnetische Südpol in der Nähe des geographischen Nordpols liegt, hat das Feld seit der Matuyama-BrunhesPolumkehr vor etwa 780000 Jahren. Davor befand es sich in ,inverser“ Polarität, während derer sich der magnetische Südpol in der Nähe des geographischen Südpols aufhält. In fernerer Vergangenheit gab es in unregelmäßigen Abständen zahlreiche weitere Polumkehrungen (Abb. 1.1). Auffallend ist die starke Variabilität der Umkehrfrequenz: Während in jüngster geologischer Vergangenheit einige Umpolungen pro Million Jahre stattfanden, blieb das Feld in der Kreidezeit über knapp 40 Millionen Jahre in der normalen Polarität. Aktualität erhält das Thema Polumkehrungen durch Spekulationen, ob die seit 150 Jahren andauernde Abnahme des Dipolmoments ein möglicher Vorläufer einer kommenden Umkehr sein könnte (Olson, 2002), da das Dipolfeld bei gleichbleibendem Trend in etwas mehr als 1000 Jahren verschwunden wäre.

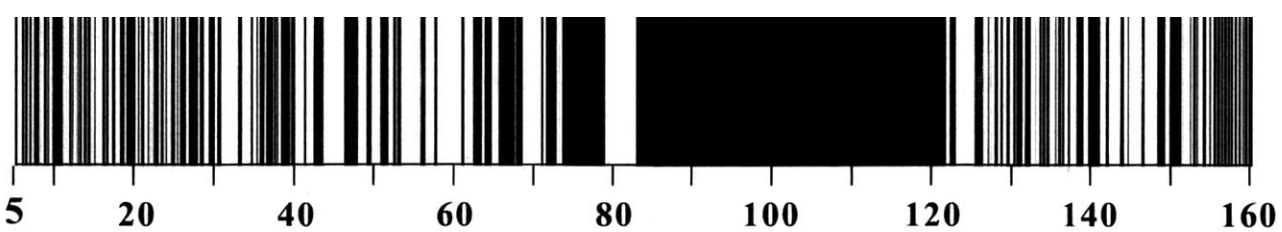

Abb. 1.1: Polarität des Erdmagnetfeldes in Abhängigkeit der Zeit von 5 bis 160 Mio. Jahre vor der Gegenwart. Schwarz normale, weiß inverse Polarität. (Goguitchaichvili et al., 2002)

Zusätzlich zu den Feldumkehrungen treten auch Exkursionen des Feldes auf. Während einer Exkursion entfernt sich die Dipolachse weit $\left(>30^{\circ}\right)$ von der Rotationsachse, kehrt dann aber in ihre ursprüngliche polnahe Lage zurück. Um die Verkippung der Dipolachse gegen die Rotationsachse zu bestimmen, braucht man eine ausreichende Kenntnis des Gesamtfeldes, d. h. es muß eine gute Abdeckung des Globus mit Meßwerten des Feldes vorliegen. Dies ist für das heutige Feld durch Satellitenmessungen gegeben und mit Einschränkungen für das historische Feld durch die Kombination der Observatoriums-Daten mit Daten aus der Seefahrt (Jackson et al., 2000). Für weiter in die Vergangenheit reichende Feldbestimmungen liegt aber zu einem Zeitpunkt oft nur eine einzelne Messung an einem einzigen Ort vor. Im Paläomagnetismus werden virtuelle geomagnetische Pole (kurz VGP's) zur Beschreibung des magnetischen Feldes benutzt. Ein VGP kann eindeutig aus der Inklination und der Deklination des Feldes bestimmt werden. Als Inklination $I$ bezeichnet man den Winkel des 
Feldvektors gegen die Horizontale, als Deklination $D$ den Winkel gegen die geographische Nordrichtung. Zusammen mit der Totalintensität $F$ wird das magnetische Feld an einem Punkt eindeutig durch seine Elemente $(F, I, D)$ beschrieben. Der VGP ist per Definition der Südpol eines geozentrischen Dipols, der am Ort der Messung (der sog. Site) die gleichen Werte für $I$ und $D$ ergibt, wie die beobachteten. Er wird als virtueller Pol bezeichnet, da er für sich betrachtet keine Aussage über die Lage des wirklichen Dipolterms des Magnetfeldes zuläßt.

Eine ausführliche Zusammenstellung des Wissens über Umpolungen und Exkursionen wird in dem Buch von Jacobs (1994) gegeben oder in etwas komprimierterer Form in den Artikeln von Merrill \& McFadden (1999) und Dormy et al. (2000). In den Artikeln werden folgende paläomagnetische Beobachtungen als robust klassifiziert:

- die bei Exkursionen und Umkehrungen beobachteten schnellen Richtungsänderungen der (virtuellen) geomagnetischen Pole finden innerhalb weniger tausend Jahre statt

- einer Polumkehr geht üblicherweise eine allmähliche Abschwächung des Feldes an der Erdoberfläche voraus, dann wandert der virtuelle geomagnetische Pol in die andere Hemisphäre, worauf die Feldintensität sich wieder erholt

- Exkursionen sind ebenso mit Phasen schwachen Feldes korreliert

- während einer Feldumkehr ist das Feld an der Erdoberfläche wahrscheinlich nichtdipolar geprägt

Das Erdmagnetfeld entsteht im äußeren, flüssigen Eisenkern der Erde in einem selbsterhaltenden Dynamoprozeß. Durch die Bewegung des leitfähigen Materials im Magnetfeld werden elektrische Ströme erzeugt, welche neues Magnetfeld erzeugen, das das schon vorhandene im Mittel konstruktiv überlagert. Die diesem Prozeß zu Grunde liegenden physikalischen Gleichungen können mit gewissen Einschränkungen numerisch simuliert werden.

In den letzten Jahren wurden viele numerische Dynamo-Modelle veröffentlicht, die grundlegende Eigenschaften des Erdmagnetfeldes reproduzieren. Die ersten selbstkonsistenten, voll dreidimensionalen Berechnungen konvektionsgetriebener Dynamos von Glatzmaier \& Roberts (1995a) und Kuang \& Bloxham (1997) erzeugten dem Erdmagnetfeld ähnelnde dipol-dominierte Magnetfelder. Die Simulationen zeigten auch weitere Eigenschaften, die als charakteristisch für das Erdmagnetfeld angesehen werden, wie z. B. die Westdrift von magnetischen Strukturen in vergleichbarer Geschwindigkeit, Bereiche niedriger Säkularvariation wie in der pazifischen Hemisphäre (Kuang \& Bloxham, 1998) und Flußbündelungen an der Kern-Mantel-Grenze (KMG) bei etwa $60^{\circ}$ Breite (Christensen et al., 1998). Trotz vieler Unterschiede zwischen den Modellen, z. B. verschiedener Approximationen der magnetohydrodynamischen (MHD) Gleichungen, unterschiedlicher Randbedingungen oder Parameterwerte, berichten diese und andere Autoren (Kagevama \& Sato, 1997a.b.c; Katavama et al., 1999; Sakuraba \& Kono, 1999; Olson et al., 1999) von einer starken Dominanz des Dipols. In fast allen Modellen richtet sich die Dipolachse bis auf wenige Grad an der Rotationsachse aus und es gibt keine Anzeichen für Feldumkehrungen.

Glatzmaier \& Roberts (1995b) berichteten als erste von einer Feldumkehr in einer numerischen Dynamo-Simulation. Mehrere Umkehrungen traten in den Modellen von Kida et al. (1997) und Kida \& Kitauchi (1998) auf, allerdings in nahezu periodischer Weise. Kageyama 
et al. (1999) beobachteten eine Umkehr des Dipolfeldes in ihrer Simulation. Sarson \& Jones (1999) untersuchten Feldumkehrungen in ihrem sogenannten 2.5-dimensionalen Modell, in welchem für die dritte Dimension nur eine nicht-axialsymmetrische Mode erhalten wird, und schlugen vor, daß sogenannte „Auftriebswellen“ in polaren Aufströmen die Feldumkehrungen auslösen. Trotz der vielen Fortschritte, die in letzter Zeit gemacht wurden, ist weder der Mechanismus einer Feldumkehr verstanden, noch ist klar, unter welchen Bedingungen solche Umkehrungen auftreten.

Von einer Ausnahme abgesehen (Kida \& Kitauchi, 1998) wurden chaotisch umkehrende dipolare Felder bisher nur in Modellen beobachtet, welche entweder Hyperdiffusivität verwenden (Glatzmaier \& Roberts, 1995a.b; Glatzmaier et al., 1999) oder unphysikalische magnetische Randbedingungen verwenden (Kagevama et al., 1999), welche das Verhalten eines Dynamos verfälschen könnten. Hyperdiffusivität bedeutet, daß die betrachtete Diffusivität stark mit dem Kugelfunktionsgrad $\ell$ anwächst, wodurch kleinskalige Strukturen effektiv gedämpft werden. Meistens wird die viskose oder magnetische Diffusivität skalenabhängig gemacht, seltener die thermische. Dieses Konzept ist hauptsächlich ein Kniff, der numerische Stabilität gewährleistet, jedoch beeinflussen die anisotropen Diffusivitäten die Dynamik der Konvektion in ungewollter Weise (Zhang \& Jones, 1997) und können unechte Feldumkehrungen auslösen, wie von Grote et al. (2000a) gezeigt wurde.

Ganz offensichtlich sind Feldumkehrungen sowie Exkursionen keine gängige Eigenschaft eines Dynamo-Modells, und es ist unklar, welche Voraussetzungen gegeben sein müssen, um solches Verhalten zu bekommen. Ein paar systematische Untersuchungen, in welchen die fundamentalen Kontrollparameter variiert wurden, ergaben keinen Hinweis auf die Frage nach Umkehrungen. Grote et al. (1999, 2000b) entdeckten mit ihren intern geheizten Modellen Bereiche im Parameterraum mit nicht-umkehrenden dipolaren, quadrupolaren und hemisphärischen Dynamos. Christensen et al. (1999) erhielten in ihren Modellen mit festem Temperaturkontrast überwiegend Lösungen, die von einem starken und stabilen Dipol dominiert wurden, und das bei ähnlichen Parameterwerten, wie Grote et al. benutzten. Daraus kann man schließen, daß die sich unterscheidenden thermischen und mechanischen Bedingungen den ausschlaggebenden Effekt hatten. Dieser Effekt wurde näher in der Studie von Kutzner \& Christensen (2000) untersucht, in welcher intern geheizte Dynamos kleinskalige Magnetfelder ohne nennenswerten Dipolanteil erzeugten, während chemisch angetriebene Konvektion das axiale Dipolfeld stabilisierte.

Der erste Teil dieser Arbeit beschäftigt sich damit, systematisch die Kontrollparameter der Dynamos zu variieren, um herauszufinden, ob in dem erreichbaren Parameterbereich Polumkehrungen auftreten. Dies soll für verschiedene Möglichkeiten, die Konvektion anzutreiben, geschehen. Im zweiten Teil werden die Auswirkungen thermischer Kern-Mantel-Kopplung auf umkehrende Dynamos untersucht.

Heterogenitäten im unteren Erdmantel, so wie sie z. B. aus der seismischen Tomographie (Su et al., 1994) abgeleitet werden können, sind Grund für laterale Variationen im Wärmefluß aus dem Kern in den Mantel. Die Mantelkonvektion (Erdplattenbewegung von etwa 5 $\mathrm{cm} / \mathrm{Jahr}$ ) findet auf wesentlich längeren Zeitskalen statt, als die viel effektivere Konvektion im flüssigen Erdkern $(\approx 10 \mathrm{~km} / \mathrm{Jahr}$, abgeleitet aus Säkularvariation), welcher an seiner äußeren Begrenzung deswegen nahezu isothermal ist. Der Mantel zeigt sich dem Kern gegenüber quasi als stationär und gibt ihm einen örtlich variierenden Wärmefluß vor. Letzterer ist höher in Bereichen kalter Mantelabströme und niedriger an Orten warmer Aufströme, 


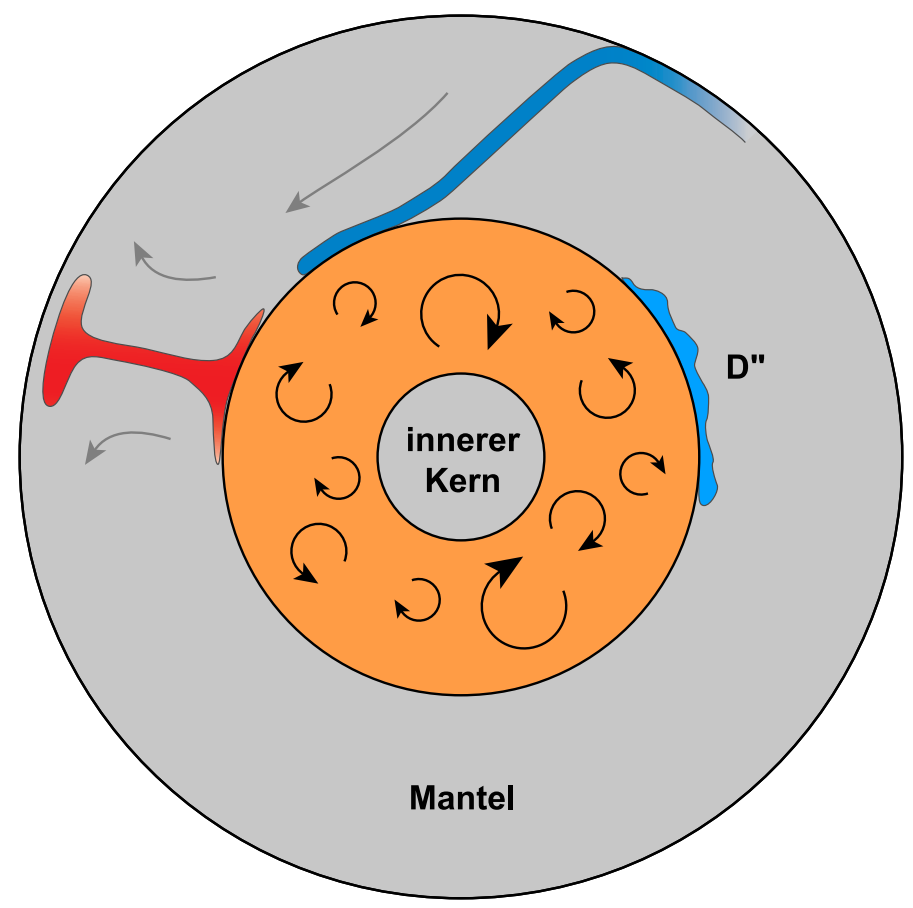

Abb. 1.2: Stark schematisierter Schnitt durch die Erde. Von der Oberfläche tauchen kalte tektonische Platten in den Mantel ab (Blau), ev. bis hinab zur KMG (van der Hilst et al.. 1998). In Blau angedeutet ist auch die sog. D"-Schicht, welche sich am Boden des Mantels durch starke Variationen der seismischen Geschwindigkeit bemerkbar macht und zu der möglicherweise Reste abgetauchter Platten beitragen (Wysession et al., 1998). Rot dargestellt ist ein warmer Aufstrom des Mantelmaterials.

wodurch der Erdkern thermisch an den Mantel gekoppelt ist (Abb. 1.2). Daß Unterschiede im Wärmefluß Auswirkungen auf das von einem Dynamo-Modell erzeugte Magnetfeld haben, wurde von Bloxham (2000) gezeigt, der eine KMG-Wärmeflußvariation von Grad 2 und Ordnung 0 vorgab, was zu einem starken Oktupolbeitrag des Magnetfeldes führte. Glatzmaier et al. (1999) zeigen, daß das verwendete Wärmeflußmuster an der KMG einen starken Einfluß auf die Umpolfrequenz ihres Dynamos hat, obwohl die Anzahl der simulierten Umkehrungen für eine statistische Auswertung zu niedrig ist, wie sie selber bemerken. Olson \& Christensen (2002) untersuchten Dynamos mit inhomogenem KMG-Wärmefluß, insbesondere ein Modell mit einem aus der seismischen Tomographie abgeleiteten Wärmeflußmuster, und fanden Flußkonzentrationen im langzeitig gemittelten Magnetfeld an Stellen, an denen auch im heutigen Feld starke Flußbündel liegen.

Mehrere paläomagnetische Studien von Feldumkehrungen und Exkursionen zeigen eine Häufung in der Verteilung der VGP's entlang amerikanischer und asiatischer Längen, und zwar nicht nur für die Umpolungen der letzten 11 Mio. Jahre (Tric et al., 1991; Lai et al.. 1991), sondern auch für die VGP's, die allein für die Matuyama-Brunhes Polumkehr an verschiedenen Orten erhalten wurden (Clement, 1991). Die Autoren weisen darauf hin, daß die Häufung der virtuellen Pole in zwei Pfaden korreliert ist mit Regionen hoher seis- 
mischer Geschwindigkeit im unteren Mantel, und daß die Pfade sehr nahe an den Längen liegen, an denen wir heute die beiden Paare kräftigen magnetischen Flusses an der KMG vorfinden. Die Beobachtungen über bevorzugte VGP-Pfade wurden als mögliche Artefakte kritisiert, weil einerseits eine viel höhere Anzahl an Meßpunkten erforderlich ist, um statistische Aussagen über VGP-Häufungen machen zu können (Valet et al., 1992) und andererseits die Verläßlichkeit von Sediment-Daten nicht gesichert ist (Langereis et al., 1992; Barton \& McFadden, 1996). Es ist fraglich, ob Sedimente die schnellen Änderungen der Feldrichtung und Intensität, die während Umpolungen auftreten, zuverlässig aufzeichnen und über lange Zeiten konservieren können. Eine unabhängige Studie mit vulkanischen Datensätzen von Umkehrungen und Exkursionen der letzten 16 Mio. Jahre zeigte allerdings eine gleichmäßige Verteilung der VGP's, woraus abzuleiten wäre, daß das umkehrende Feld statistisch axialsymmetrisch ist und insbesondere nicht von thermischen Anomalien des unteren Mantels beeinflußt wird (Prévot \& Camps, 1993). Im Gegensatz dazu steht die Untersuchung von Love (1998), der auch in vulkanischen Datensätzen (der letzten 20 Mio. Jahre) deutliche Hinweise auf bevorzugte VGP-Längen fand.

Coe et al. (2000) untersuchten vier simulierte Polumkehrungen im Detail. Zwei entstammten einem Modell mit homogenem KMG-Wärmefluß, zwei einem Modell mit einem aus der seismischen Tomographie abgeleiteten Wärmeflußmuster. Die beiden Umkehrungen aus dem tomographischen Modell legen eine grobe Korrelation der Dichten der VGP's mit Regionen überdurchschnittlich hohen Wärmeflusses nahe, was aber auch Zufall sein könnte.

Die Frage, ob die real beobachteten Häufungen von VGP's echt oder nur ein Artefakt sind, ist also offen. Mithilfe der Simulationen, die umkehrende Felder erzeugen, soll deswegen untersucht werden, ob eine inhomogene thermische Randbedingung an der äußeren Grenze tatsächlich zu beobachtbaren VGP-Häufungen führt. 


\section{Das numerische Modell}

Für die Simulationen wird ein numerisches Modell benutzt, welches ursprünglich von Glatzmaier (1984) für stellare Dynamos entwickelt, dann von Olson \& Glatzmaier (1995) für Magnetokonvektion und schließlich (Christensen et al., 1998, 1999; Olson et al., 1999) für die Simulation selbsterhaltender Dynamos modifiziert wurde. Der Code wurde mithilfe eines Benchmark-Tests verifiziert (Christensen et al., 2001).

Es werden die dreidimensionalen, zeitabhängigen Gleichungen für Konvektion und Magnetfelderzeugung in einer mit einer leitfähigen Flüssigkeit gefüllten, rotierenden Kugelschale im Rahmen der Boussinesq-Approximation gelöst. Im Boussinesq-Modell wird die Dichte $\rho$ als konstant angenommen, nur nicht im Auftriebsterm, wo sie variabel bleibt und von der Temperatur oder der Zusammensetzung des Materials abhängen kann. Sowohl der Mantel als auch der innere Kern werden als elektrisch isolierend angenommen. Hollerbach \& Jones (1993) und Gubbins (1999) wiesen darauf hin, daß ein leitfähiger innerer Kern einen großen Einfluß auf das Umkehrverhalten eines Dynamos haben kann. Die beschränkte Zerfallszeit des magnetischen Feldes im inneren Kern sollte insbesondere zu häufige Umkehrungen verhindern. Wicht (2002) verglich Simulationen mit und ohne innerem Kern im gleichen Parameterbereich, der auch hier verwendet wird, fand allerdings kaum Unterschiede.

Eine Skizze der Kugelschale mit einigen Bezeichnungen ist in Abb. 2.1 gezeigt. Der Abstand zwischen äußerer und innerer Begrenzung ist im numerischen Modell auf 1 normiert,

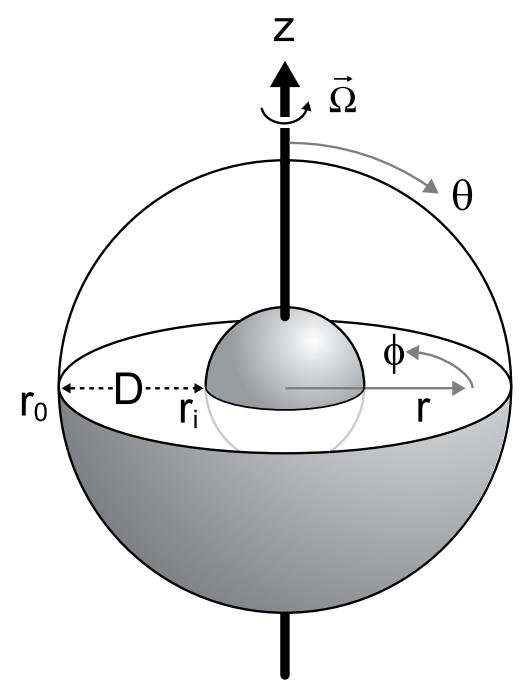

Abb. 2.1: Schematische Darstellung der Modellgeometrie. 
$r_{0}-r_{i}=1$. Das Verhältnis des Radius des inneren Kerns $r_{i}$ zum Radius des äußeren Kerns $r_{0}$ wird (mit Ausnahme der Modelle aus Abschnitt 3.1) entsprechend dem Radienverhältnis der Erde gewählt:

$$
\frac{r_{i}}{r_{0}}=\frac{1220 \mathrm{~km}}{3480 \mathrm{~km}}=0.3505747=r_{q}
$$

\subsection{Magneto-hydrodynamische Gleichungen}

Es werden die MHD-Gleichungen in dimensionsloser Form gelöst. Die Bewegung der Flüssigkeit im äußeren Kern wird von der Navier-Stokes-Gleichung beschrieben (zur Herleitung siehe z. B. Batchelor (1967)):

$$
\frac{\mathrm{E}}{\mathrm{qPr}}\left(\frac{\partial \mathbf{u}}{\partial t}+\mathbf{u} \cdot \nabla \mathbf{u}\right)+2 \hat{\mathbf{z}} \times \mathbf{u}+\nabla P=\mathrm{E} \nabla^{2} \mathbf{u}+\operatorname{Raq} \operatorname{Pr} \frac{\mathbf{r}}{r_{o}} T+(\nabla \times \mathbf{B}) \times \mathbf{B}
$$

Mit der Flüssigkeitsgeschwindigkeit $\mathbf{u}$, der Zeit $t$, der magnetischen Induktion $\mathbf{B}$ und dem Druck $P$. Dabei ist $\hat{\mathbf{z}}$ der Einheitsvektor in Richtung der Rotationsachse und $\mathbf{r}$ der Radiusvektor. $T$ kann entweder für die Temperatur oder für die Konzentration eines leichten Elementes stehen. Hinzu kommt die vereinfachte Kontinuitätsgleichung für eine BoussinesqFlüssigkeit:

$$
\nabla \cdot \mathbf{u}=0
$$

Die enormen Drücke im Inneren der Erde haben natürlich eine Auswirkung auf die Dichte, die sich allerdings kaum auf die Dynamik der Flüssigkeit auswirkt. Eine inkompressible Theorie zu benutzen bedeutet, daß die Variationen der Variablen als Variationen um den adiabatischen Referenzzustand zu verstehen sind.

Auftriebsvariationen werden von der folgenden Gleichung beschrieben:

$$
\frac{\partial T}{\partial t}+\mathbf{u} \cdot \nabla T=\mathrm{q} \nabla^{2} T+S
$$

Der Term $S$ steht für volumetrische Quellen oder Senken des Auftriebs. Erzeugung und Zerfall des Magnetfeldes wird durch die magnetische Induktionsgleichung beschrieben:

$$
\frac{\partial \mathbf{B}}{\partial t}=\nabla \times(\mathbf{u} \times \mathbf{B})+\nabla^{2} \mathbf{B}
$$

Sie leitet sich her aus den Maxwell-Gleichungen und dem verallgemeinerten Ohmschen Gesetz, $\mathbf{j}=\sigma(\mathbf{E}+\mathbf{v} \times \mathbf{B})$, siehe z. B. Fearn (1998). $\sigma$ ist die elektrische Leitfähigkeit und $\mathbf{E}$ das elekrisches Feld. Dabei wird die sog. magneto-hydrodynamische Approximation benutzt, bei der die freie Ladungsdichte und der elektrische Verschiebungsstrom in den Maxwellgleichungen vernachlässigt werden. Das gilt, solange die auftretenden Flüssigkeitsgeschwindigkeiten klein gegen die Lichtgeschwindigkeit $c$ bleiben, $|\mathbf{v}| \ll c$, was für die Erde selbstverständlich erfüllt ist. In der Magneto-Hydrodynamik ist es üblich, $\mathbf{B}$ als magnetisches Feld zu bezeichnen, obwohl $\mathbf{B}$ formal richtig magnetische Induktion heißt.

Die Skalierungen für Länge, Zeit und magnetische Induktion sind $D, D^{2} / \eta$, und $\left(\rho \eta \Omega \mu_{0}\right)^{1 / 2}$. Dabei ist $D$ die Schalendicke, $\eta$ die magnetische Diffusivität, $\rho$ die Dichte und $\Omega$ die (konstante) Rotationsrate um die z-Achse, s. Abb. 2.1. Wie in rotierenden Systemen 
üblich wird hier die modifizierte Rayleighzahl benutzt, in welche die Rotationsrate $\Omega$ eingeht. Das ist sinnvoll, weil die Konvektion hauptsächlich durch die Rotation erschwert wird. Damit sind die vier dimensionslosen Eingabeparameter:

$$
\begin{aligned}
& \text { Prandtlzahl } \quad \operatorname{Pr}=\frac{\mathrm{v}}{\kappa} \\
& \text { Ekmanzahl } \quad \mathrm{E}=\frac{\mathrm{v}}{\Omega D^{2}} \\
& \text { modifizierte Rayleighzahl } \quad \mathrm{Ra}=\frac{g_{o} \alpha \Delta T_{\mathrm{kond}} D}{\mathrm{v} \Omega} \\
& \text { Robertszahl } \quad \mathrm{q}=\frac{\kappa}{\eta}
\end{aligned}
$$

Es ist $v$ die kinematische Viskosität, $\kappa$ die thermische Diffusivität, $\alpha$ der thermische Expansionskoeffizient, $g_{o}$ die Schwerebeschleunigung an der äußeren Grenze (der KMG) und $\Delta T_{\text {kond }}$ die Differenz von $T$ zwischen der inneren und äußeren Grenze im konduktiven Fall. Bei chemisch angetriebener Konvektion ist $\kappa$ als chemische Diffusivität zu verstehen und $\alpha$ als ein Koeffizient, der die Dichte der Flüssigkeit in Abhängigkeit der Konzentration einer leichten Komponente beschreibt. $\Delta T_{\text {kond }}$ wird entweder als fester Temperaturkontrast vorgegeben oder wird aus der vorgegebenen Verteilung der Quellen und Senken für den konduktiven Fall berechnet.

\subsection{Antrieb der Konvektion}

Als Antrieb der Konvektion im äußeren Erdkern werden üblicherweise thermische und chemische Effekte in Betracht gezogen (Fearn, 1998). Thermischer Antrieb entsteht durch die säkulare Abkühlung der Erde und durch den Wärme erzeugenden Zerfall eines im Kern verteilten radioaktiven Elementes $\left({ }^{40} \mathrm{~K}\right)$. Chemischer Antrieb entsteht durch das leichte Material, welches bei der Auskristallisation des inneren Kerns freigesetzt wird (Loper \& Roberts, 1981). Der äußere Erdkern besteht aus flüssigem Eisen plus einem Bruchteil einer darin gelösten leichten Komponente; das könnten Elemente wie Schwefel, Silizium oder Sauerstoff sein (Poirer, 1994). Weil die leichte Komponente eine geringere Löslichkeit in der festen Phase hat, wird sie beim Wachsen des inneren Kerns in den äußeren Kern freigesetzt. Durch das Wachsen des inneren Kerns wird einerseits durch die freigesetzte Kristallisationswärme Auftrieb erzeugt, andererseits aber auch durch das freiwerdende Material selbst, welches eine geringe Dichte hat. Nach Lister \& Buffett (1995) sind etwa 80\% des Auftriebs chemischen Ursprungs, die Unsicherheiten sind aber groß. Die chemische und zwei thermische Antriebsarten und ihre Auswirkungen auf die Konvektion und das Magnetfeld eines Dynamos werden ausführlich in Kutzner (1999) untersucht.

Auch in den hier behandelten Modellen kann die Konvektion auf verschiedene Weise in Gang gehalten werden, zunächst entweder rein thermisch oder rein chemisch. Im einfachsten Falle wird sie thermisch angetrieben durch Vorgabe eines festen Temperaturkontrastes zwischen innerer und äußerer Begrenzung (Antriebstyp 1), so wie beispielsweise bei Christensen et al. (1999) oder Kida \& Kitauchi (1998). Alternativ können auch im Volumen homogen verteilte Wärmequellen (ähnlich wie bei Grote et al. (1999) oder Jones et al. (1995)) oder feste Wärmeflüsse an einer oder beiden Begrenzungen vorgegeben werden. Glatzmaier 


\begin{tabular}{llllr} 
Typ & Antriebsart & Randbedingung für $T$ & $S$ \\
& & IKG & KMG & \\
\hline 1 & Festes $\Delta T$ & $T=1$ & $T=0$ & 0.0 \\
2 & Interne Heizung / säkulare Abkühlung & $\partial T / \partial r=0$ & $T=0$ & 1.0 \\
3 & Fester Fluß IKG mit 50\% int. Heizung & $\partial T / \partial r=$ const & $T=0$ & 0.5 \\
4 & Fester Fluß IKG mit 10\% int. Heizung & $\partial T / \partial r=$ const & $T=0$ & 0.1 \\
5 & Fester Fluß IKG & $\partial T / \partial r=$ const & $T=0$ & 0.0 \\
6 & Fester Fluß an beiden Grenzen & $\partial T / \partial r=$ const & $\partial T / \partial r=$ const & 0.0 \\
7 & Fester Fluß KMG & $T=1$ & $\partial T / \partial r=$ const & 0.0 \\
8 & Chemische Konvektion & $T=1$ & $\partial T / \partial r=0$ & -1.0 \\
9 & Chem. Konvektion mit festem Fluß IKG & $\partial T / \partial r=$ const & $\partial T / \partial r=0$ & -1.0 \\
\hline
\end{tabular}

Tab. 2.1: Untersuchte Antriebsarten für die Konvektion: Thermische (Typ 1 - 7) oder chemische (Typ 8 - 9) Randbedingungen und Werte des volumetrischen Quell- oder Senkenterms $S$.

\& Roberts (1995a) und Kuang \& Bloxham (1997) modellieren z. B. thermische Konvektion mit einer vorgegebenen Wärmeflußdichte als Randbedingung an der Grenze zum inneren Kern (IKG). Es werden verschiedene Kombinationen von thermischen Randbedingungen und unterschiedlich große Beiträge der internen Wärmequellen behandelt, was über die ursprüngliche Untersuchung von Kutzner (1999) hinausgeht.

In Tabelle 2.1 finden sich die Randbedingungen für $T$ und Angaben über den Wert des Terms $S$ der internen Quellen oder Senken für die untersuchten Antriebtypen. In den Modellen 2 - 5 wird die Temperatur an der äußeren Grenze konstant gehalten, während die interne Heizrate $S$ variiert wird. Interne Heizung und säkulare Abkühlung sind formal äquivalent. In Typ 2 wird die gesamte Wärme im Volumen $V$ des äußeren Kerns produziert, in Typ 3 wird die eine Hälfte durch Quellen in $V$ produziert, während die andere Hälfte als Wärmefluß von der IKG kommt. Die zeitgemittelten Wärmeflüsse an der äußeren Grenze sind für diese vier Modelle gleich.

Weinstein \& Olson (1990) zeigten, daß ein volumetrischer Senkenterm (negatives $S$ ) zusammen mit einer Null-Fluß Randbedingung an der KMG geeignet ist, rein chemisch angetriebene Konvektion zu modellieren. Es wird für die folgenden Untersuchungen angenommen, daß die durch den Kern transportierte Wärme genau dem adiabatischen Wärmefluß entspricht, so daß die Konvektion allein aufgrund der chemisch erzeugten Dichteunterschiede angetrieben wird. Es sei $C$ die Konzentration des leichten Elements. Der Transport von $C$ wird durch folgende Gleichung beschrieben:

$$
\frac{\partial C}{\partial t}+\mathbf{u} \cdot \nabla C=\kappa \nabla^{2} C
$$

Da die leichte Komponente den Kern nicht verlassen kann, muß an der äußeren Grenze eine Null-Fluß-Randbedingung gelten. $C$ wird in einen mittleren Anteil $C_{0}$ und einen variierenden Anteil $T$ aufgeteilt:

$$
C(r, \theta, \phi, t)=C_{0}(t)+T(r, \theta, \phi, t) .
$$


Weil die leichte Komponente ständig an der IKG freigesetzt wird, gibt es ein allmähliches Anwachsen von $C_{0}$ mit der Zeit. Der Einfachheit halber wird lineares Anwachsen angenommen:

$$
C_{0}(t)=C_{0}(0)+\gamma t \text {. }
$$

Durch Einfügen der Gleichungen 2.10 und 2.11 in 2.9 erhält man einen effektiven Senkenterm in der Gleichung für den Transport von $T$ :

$$
\frac{\partial T}{\partial t}+\mathbf{u} \cdot \nabla T=\kappa \nabla^{2} T-\gamma .
$$

Die skalierte Form von Gleichung 2.12 ist formal äquivalent zur skalierten Wärmeleitungsgleichung 2.3 mit negativem $S$.

\subsection{Numerische Methode}

Es wird eine modifizierte Version des Codes benutzt, der ausfürhlich in Glatzmaier (1984) beschrieben ist. Wegen $\nabla \cdot \mathbf{B}=0$ kann das Magnetfeld als Rotation eines Vektorpotentials A dargestellt werden, $\mathbf{B}=\nabla \times \mathbf{A}$. Die allgemeine Form eines Vektorpotentials in Kugelkoordinaten ist $\mathbf{A}=\nabla \Phi+j \mathbf{r}+\nabla \times(b \mathbf{r})$ mit den Skalarfeldern $\Phi, j$ und $b$. Das ergibt

$$
\begin{aligned}
\mathbf{B} & =\nabla \times(j \mathbf{r})+\nabla \times \nabla \times(b \mathbf{r}) \\
& =\mathbf{B}_{\mathrm{tor}}+\mathbf{B}_{\mathrm{pol}}
\end{aligned}
$$

Weil die Rotation einer Divergenz verschwindet, entfällt der $\Phi$-Term. Das sich aus toroidalem $\mathbf{B}_{\text {tor }}$ und poloidalem $\mathbf{B}_{\text {pol }}$ Anteil zusammensetzende Magnetfeld wird durch die beiden Skalarfelder $j$ und $b$ dargestellt. Wegen 2.2 kann analog mit dem Geschwindigkeitsfeld verfahren werden. Durch die Zerlegung der Felder $\mathbf{B}$ und $\mathbf{u}$ in ihre toroidalen und poloidalen Teile ist $\nabla \cdot \mathbf{B}=0$ sowie die Kontinuitätsgleichung 2.2 immer automatisch erfüllt.

Die toroidalen und poloidalen Skalarpotentiale für das Geschwindigkeits- und Magnetfeld werden zusammen mit dem Temperatur- (oder Konzentrations-) und Druckfeld in den Winkelvariablen $\theta$ und $\phi$ als Kugelfunktionen $Y_{l m}$ und in radialer Richtung $r$ als ChebyshevPolynome $T_{n}$ entwickelt.

Mit 2.13 ergeben sich die drei Komponenten des Magnetfeldes zu

$$
\begin{aligned}
& B_{r}=\frac{1}{r^{2}} \sum_{\ell, m} \ell(\ell+1) b_{\ell m} Y_{\ell m} \\
& B_{\theta}=\frac{1}{r \sin \theta} \sum_{\ell, m}\left(\frac{\partial b_{\ell m}}{\partial r} \sin \theta \frac{\partial Y_{\ell m}}{\partial \theta}+j j_{\ell m} \frac{\partial Y_{\ell m}}{\partial \phi}\right) \\
& B_{\phi}=\frac{1}{r \sin \theta} \sum_{\ell, m}\left(\frac{\partial b_{\ell m}}{\partial r} \frac{\partial Y_{\ell m}}{\partial \phi}-j_{\ell m} \sin \theta \frac{\partial Y_{\ell m}}{\partial \theta}\right)
\end{aligned}
$$

Die Entwicklung der komplexen Koeffizienten der $Y_{\ell m}$ in Chebychev-Polynome $T_{n}$ ist am Beispiel von $b_{\ell m}$ :

$$
b_{\ell m}(r, t)=\left(\frac{2}{N}\right)^{1 / 2} \sum_{n=0}^{N} b_{\ell m}^{n}(t) T_{n}(r)
$$


Der Doppelstrich an der Summe kennzeichnet, daß die Terme für $n=0$ und $n=N$ mit $1 / 2$ multipliziert werden.

Die nichtlinearen Terme der Gleichungen (Advektion und Lorentzkräfte) und die Corioliskraft werden explizit behandelt und auf einem Gitter im physikalischen Raum berechnet. Die $N+1$ radialen Gitterpunkte liegen bei

$$
r_{n}=\frac{r_{0}+r_{i}}{2}+\cos \frac{n \pi}{N} \quad n \in 0 \ldots N
$$

also an den Rändern etwas dichter. Die Transformation zwischen dem Raumgitter und dem Spektralraum wird aliasfrei durchgeführt. Für jede harmonische Mode wird die radiale Variation mit einer Kollokationsmethode berechnet, welche auf die linearen Terme der Gleichungen wirkt, und die sechs skalaren Variablen werden in jedem Zeitschritt durch ein Adams-Bashforth Schema zweiter Ordnung integriert. Die Zeitschrittlänge wird dynamisch durch ein Courant-Kriterium beschränkt, in welches sowohl die Flüssigkeitsgeschwindigkeit (wegen der expliziten Behandlung des Advektionsterms) als auch eine modifizierte AlfvénGeschwindigkeit eingeht (wegen der expliziten Behandlung der Lorentzkraft). Es werden keine Hyperdiffusivitäten eingesetzt. Eine detaillierte Beschreibung findet sich in Glatzmaier (1984) und Olson \& Glatzmaier (1995), sowie in Christensen et al. (1999) bezüglich der Zeitschrittkontrolle.

\subsection{Rand- und Anfangsbedingungen}

Die thermischen Randbedingungen sind durch die Wahl der Antriebsart festgelegt (Tab. 2.1). Da Mantel und innerer Kern fest sind, wird verlangt, daß die Geschwindigkeit an beiden Grenzflächen auf Null abnimmt; diese Wahl der mechanischen Randbedingung wird auch als „feste Ränder“ bezeichnet:

$$
\mathbf{u}=0 \quad \text { bei } r=r_{i} \text { und } r=r_{0}
$$

An den Rändern muß zusätzlich aufgrund der Annahme eines elektrisch nichtleitenden inneren Kerns und Mantels das toroidale Magnetfeld verschwinden und das poloidale einem Potentialfeld gleichen:

$$
\begin{aligned}
& \mathbf{B}_{\mathrm{pol}}=-\nabla \Phi \quad \text { bei } r=r_{i} \text { und } r=r_{0} \\
& \text { mit } \quad \nabla^{2} \Phi=0 \quad \text { wegen } \nabla \cdot \mathbf{B}=0
\end{aligned}
$$

Falls nicht ausdrücklich anders erwähnt, wurden die hier durchgeführten Simulationen mit den Ergebnissen bereits bestehender Modell-Läufe gestartet. Man bevorzugt als Anfangszustand eine Lösung, die im Parameterraum nicht zu weit entfernt ist. Dies verkürzt den transienten Zustand, in dem der Dynamo sich auf die geänderten Parameter einstellt. Um zu beurteilen, wann ein Modell sein statistisches Gleichgewicht erreicht hat, werden die Zeitreihen der kinetischen und magnetischen Energien beobachtet. Für die Berechnung von zu vergleichenden Größen wie z. B. mittleren Energiedichten wird die anfängliche transiente Periode natürlich ausgeschlossen.

$\mathrm{Da}$ die gefundene Lösung von der Anfangsbedingung völlig unabhängig wird, wenn man nur lange genug integriert, wird zwar erhofft, kann aber nie mit Sicherheit erwartet werden. 
Die meisten der hier durchgeführten Simulationen sind aber aufgrund der hohen Rayleighzahl so chaotisch, daß man annehmen darf, daß die Erinnerung eines Dynamos an seinen Anfangszustand schnell verloren geht. Für ein Modell mit Parametern, die nahe an der Grenze zwischen dipol-dominierten und schwach-dipolaren Dynamos liegen (Antriebstyp 4 mit $\mathrm{E}=3 \times 10^{-4}, \mathrm{Ra}=585, q=3$ ), wurde verifiziert, daß die gefundene Lösung nicht von der Anfangsbedingung abhängig ist. Diese war in einem Fall ein dipol-dominierter Dynamo bei niedriger Ra, im anderen Fall ein chaotisch umkehrender Dynamo mit geringem Dipolanteil. Nach zwei bzw. fünf magnetischen Diffusionszeiten ergab sich in beiden Fällen einen Dynamo mit stabilem, dipol-dominierten Feld und nahezu gleichen zeitgemittelten kinetischen und magnetischen Energien.

\subsection{Numerische Auflösung}

Christensen et al. (1999) schlugen als Faustregel vor, daß eine Dynamosimulation gut aufgelöst ist, wenn die kinetischen und magnetischen Energien um mehr als den Faktor 100 vom spektralen Maximum bis zur kleinsten aufgelösten Wellenlänge abfallen. Doch sogar wenn die Auflösung so gering war, daß dieses Kriterium verletzt wurde, stellten sie fest, daß die zeitgemittelten Gesamtenergien und Energieverteilungen auf die unterschiedlichen Moden in der Nähe des spektralen Maximums sehr ähnlich waren.

Für die kinetischen Energien und für die magnetischen Energien bei kleinen bis moderaten Ra wird dieses Kriterium hier immer erfüllt. Leider fallen aber die magnetischen Energien der Dynamos bei hohen Ra so langsam mit dem harmonischen Grad $\ell$ ab, daß sogar bei Auflösungen bis $\ell_{\max }=85$, welche die Grenze des Machbaren für diese Berechnungen ist, dieses Faktor-100 Kriterium nicht erfüllt werden kann.

Um herauszufinden, ob diese Beschränkung der Auflösung einen gravierenden Einfluß auf die Lösung hat, wurde ein Auflösungstest für den Fall mit der höchsten Ra durchgeführt. Abb. 2.2 zeigt, daß die Strukturen im Magnetfeld mit großer bis mittlerer Wellenlänge bis ungefähr $\ell=\ell_{\max } / 2$ nicht durch das Abschneiden des Spektrums bei $\ell_{\max }$ beeinflußt werden. Das Maximum des Spektrums stimmt für alle Auflösungen gut überein, lediglich die gesamte magnetische Energie wird um etwa $25 \%$ überschätzt im Falle der gröbsten Auflösung $\ell_{\max }=$ 42.

Es wird eine Kugelfunktionsentwicklung mit $\ell_{\max } \geq 64$ und Chebychev-Entwicklungen bis Grad 39 oder 47 in allen Fällen mit hoher Rayleighzahl benutzt. Bei $\ell_{\max }=85$ werden dabei im physikalischen Raum 128 Punkte in $\theta$ - und 256 Punkte in $\phi$-Richtung verwendet; bei einer typischen Chebychev-Entwicklung bis Grad 39 werden 41 Punkte in radialer Richtung verwendet. 


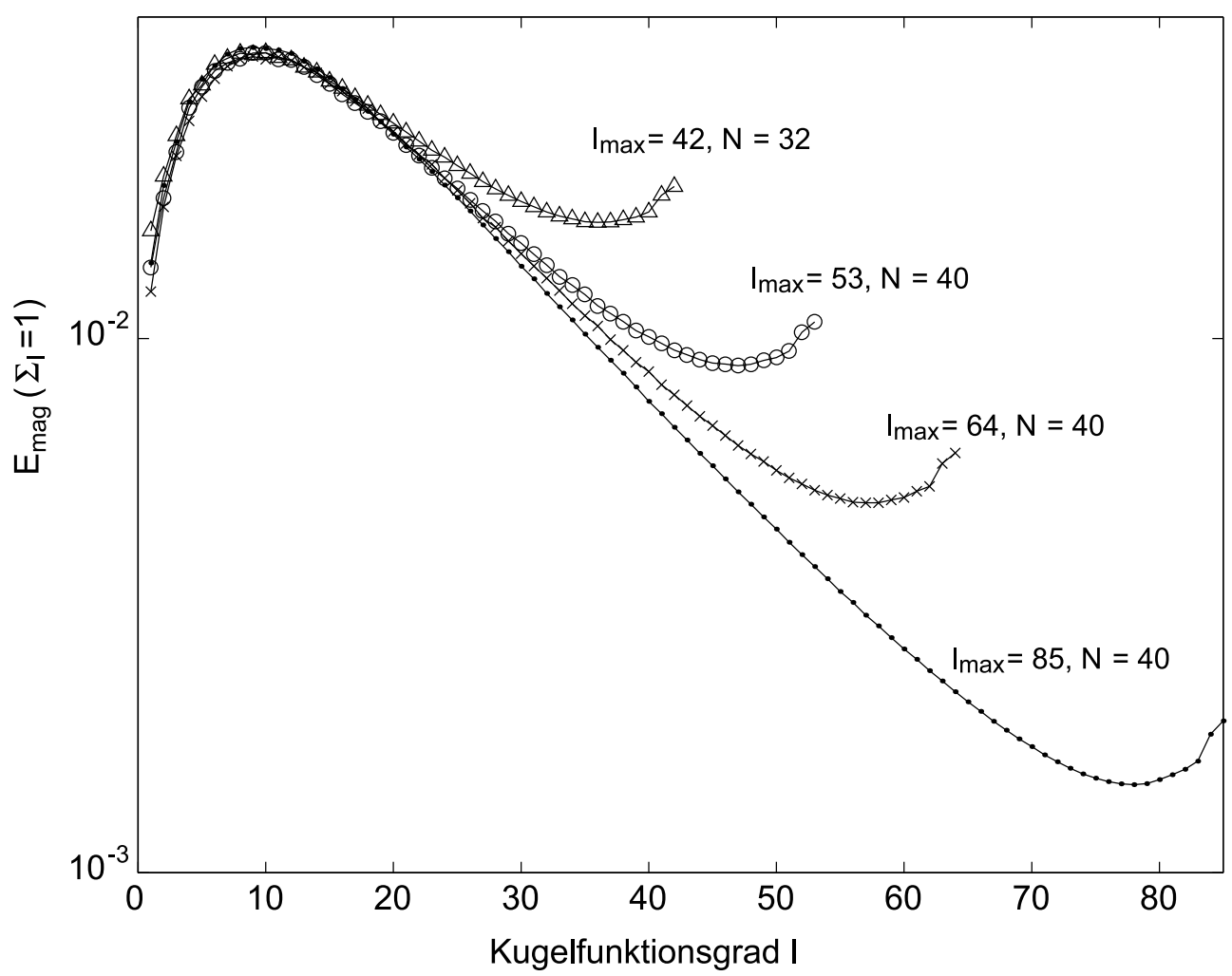

Abb. 2.2: Auflösungstest für den Fall mit der höchsten Rayleighzahl, i.e. chemische Konvektion (Typ 8) bei $\mathrm{Ra}=38 \times \mathrm{Ra}_{\mathrm{krit}}, \mathrm{E}=3 \times 10^{-4}, \mathrm{q}=3$. Zeitgemittelte Spektren der magnetischen Energie als Funktion des Kugelfunktionsgrades $\ell$. Die Spektren sind mit der Gesamtenergie normiert. 


\section{Der Parameterbereich für umkehrende Dynamos}

Ein großes Zugeständnis, welches man bei der numerischen Lösung des Dynamo-Problems machen muß, ist, daß einige physikalische Parameter nicht auf die im Erdkern geltenden Werte gesetzt werden können. Im dimensionslosen Fall heißt das, daß im wesentlichen die Ekmanzahl, welche das Verhältnis von viskosen Kräften zu Corioliskräften angibt, nicht angemessen klein $\left(\leq 10^{-9}\right)$ gewählt werden kann. Die niedrigsten Werte für E, die mit heutigen Supercomputern erreichbar sind, liegen für voll dreidimensionale MHD Simulationen ohne Hyperdiffusivitäten bei etwa $10^{-5}$ (Olson \& Christensen, 2002; Glatzmaier, 2002: Kono \& Roberts. 2002).

Durch die explizite Behandlung der Corioliskraft wird die maximale Zeitschrittlänge $\delta t$ durch die Ekmanzahl beschränkt ( $\delta t<\mathrm{E} / 4)$. Bei den Flüssigkeitsgeschwindigkeiten, wie sie bei stark überkritischen Rayleighzahlen auftreten, ist das stärker begrenzende Kriterium jedoch meist das Courant-Kriterium: Ein Flüssigkeitselement darf sich in einem Zeitschritt nicht weiter als einen Gitterpunkt fortbewegen. Da einerseits die Konvektion bei kleinen Ekmanzahlen immer kleinskaliger wird und andererseits die Grenzschichten (z. B. an den Rändern, an denen die Geschwindigkeit auf Null abfallen muß) immer dünner werden, muß das numerische Gitter immer feiner gewählt werden, je kleiner die Ekmanzahl. Die Anzahl an Rechenoperationen pro Zeitschritt wird dadurch wesentlich erhöht, wobei gleichzeitig noch der Zeitschritt kleiner wird.

Die zweite dimensionslose Zahl, die in den Modellen weit vom wahren Wert abweicht, ist die Robertszahl, welche für die Erde bei etwa $10^{-5}$ liegt $\left(\kappa=10^{-5} \mathrm{~m}^{2} / \mathrm{s}, \eta=1 \mathrm{~m}^{2} / \mathrm{s}\right.$, Werte aus Gubbins \& Roberts (1987)). Für $\mathrm{q} \ll 1$ lassen sich bei Ekmanzahlen von der Größenordnung $10^{-4}$ jedoch noch keine selbsterhaltenden Magnetfelder finden. Je kleiner E, desto kleiner ist aber die kritische Robertszahl, bei der selbsterhaltende Dynamowirkung einsetzt (Christensen et al., 1999), so daß sich dieses Problem für kleinere Ekmanzahlen selbst löst.

Die Prandtlzahl kann problemlos auf den für die Erde abgeschätzten Wert von 0.1 bis 1 gesetzt werden, deswegen wird sie hier auch nicht weiter variiert. Der Einfluß der Prandtlzahl auf Dynamos wurde von Oleiniczak (2002) untersucht.

Die Rayleighzahl für den Erdkern abzuschätzen ist schwer, da man dazu wissen müßte, wie stark überadiabatisch der Temperaturgradient im Kern ist und in welchem Verhältnis chemische und thermische Effekte zur Konvektion beitragen. Wahrscheinlich liegt Ra weit über ihrem kritischen Wert, immerhin gibt es Flüssigkeitsbewegungen, die stark genug sind, ein Magnetfeld aufrecht zu erhalten.

Man kann zwar nicht darauf hoffen, in absehbarer Zeit realistische Werte für die Ekman- 
zahl einsetzen zu können, aber man kann den erreichbaren Parameterbereich erforschen und daraus Erklärungen für die beobachteten Eigenschaften des Erdmagnetfeldes ableiten. Obwohl der Einfluß der Viskosität in den Modellen enorm überbewertet wird (oder sie zu langsam rotieren, wenn man so will), stimmen die beiden wichtigsten Kräfteverhältnisse mit den Werten für die Erde überein. Die magnetische Reynoldszahl gibt das Verhältnis der Advektion zur Diffusion in der Induktionsgleichung an:

$$
\mathrm{Rm}=\frac{u D}{\eta}
$$

Sie liegt für den Erdkern bei etwa 200 (Flüssigkeitsgeschwindigkeit im Kern $u \approx 10^{-4} \mathrm{~m} / \mathrm{s}$ ). Die Elsasserzahl, das Verhältnis von Lorentz- zu Corioliskräften, ist

$$
\Lambda=\frac{B^{2}}{\rho \mu \eta \Omega}
$$

und damit von der Größenordnung 1 (Gubbins \& Roberts, 1987). Bei der hier gewählten Skalierung für $B$ ist das Quadrat des dimensionslosen Magnetfeldes gleich der lokalen Elsasserzahl. Sowohl Rm als auch $\Lambda$ sind Ergebnisse der Modelle.

Zunächst sollen die Bedingungen gefunden werden, unter denen Umkehrungen stattfinden. Dazu werden drei parallele Strategien verfolgt:

- Es soll versucht werden, den Dynamo von Kida \& Kitauchi (1998) zu reproduzieren. Dieser zeigt Umkehrungen des Dipolfeldes, welches im Verhältnis zu den übrigen Beiträgen allerdings schwach ist. Durch Variation der Parameter könnte man möglicherweise einen umkehrenden Dynamo mit stärkerem Dipolbeitrag erhalten (Kap. 3.1.

- Der Parameterbereich (E, q, Ra) wird systematisch erforscht und insbesondere zu hohen Rayleighzahlen hin erweitert, da sich mit heftiger werdender Konvektion i. a. auch die Zeitabhängigkeit eines Systems erhöht (Kap. 3.2).

- Es werden dafür viele verschiedene Antriebsarten getestet. Da reine interne Heizung kleinskalige Magnetfelder ohne nennenswerten Dipolanteil generiert Kutzner \& Christensen. 2000), ist denkbar, daß ein gewisser Anteil interner Wärmequellen eine sonst stabile Lösung zum Umkehren anregt (Kap. 3.3).

Für die Simulationen im Rahmen der Parameterstudie werden verschiedene zeitgemittelte Kenndaten ermittelt. Die magnetische Reynoldszahl wird basierend auf der rmsGeschwindigkeit in der Kugelschale berechnet. Die Nusseltzahl Nu ist in Modell Typ 1 definiert als Wärmefluß durch die KMG relativ Wärmefluß im konduktiven Fall. In den anderen Modelltypen wird sie definiert als

$$
\mathrm{Nu}=\frac{\Delta T_{\text {kond }}}{\Delta T_{\text {konv }}}
$$

wobei $\Delta T_{\text {konv }}$ der zeitgemittelte Unterschied von $T$ zwischen der inneren und der äußeren Grenze ist. Die magnetische Energiedichte in der Kugelschale ist gegeben durch

$$
E_{\mathrm{mag}}=\frac{1}{2 V_{s} \mathrm{E} \mathrm{q} \mathrm{Pr}} \int_{V_{s}} \mathbf{B}^{2} d V
$$


und die kinetische Energiedichte als

$$
E_{\text {kin }}=\frac{1}{2 V_{s}(\mathrm{q} \operatorname{Pr})^{2}} \int_{V_{s}} \mathbf{u}^{2} d V
$$

wobei $V_{s}$ das Volumen der Kugelschale darstellt. Zeitgemittelte Werte werden mit einem Überstrich gekennzeichnet. Zusätzlich zur volumengemittelten magnetischen Energie wird die rms-Intensität des Magnetfeldes an der äußeren Grenze $\bar{B}_{\text {KMG }}$ berechnet. Weil die Stärke des magnetischen Dipols relativ zum Gesamtfeld von besonderem Interesse ist, wird der Beitrag des Dipols zur magnetischen Energiedichte in der Kugelschale, $\bar{E}_{\text {mag }}^{\ell=1}$, und zur Feldstärke an der KMG, $\bar{B}_{\text {dip }}$, berechnet. Generell stimmt der Relativbeitrag des Dipols zur KMG-Feldstärke mit dem Beitrag des $\ell=1$-Terms zur poloidalen magnetischen Energie im Volumen überein. Für die Fälle, die ein nicht-umkehrendes Dipolfeld generieren, ist schließlich noch die durchschnittliche Neigung des externen Dipolfeldes gegen die Rotationsachse angegeben.

\subsection{Umkehrende Dynamos bei hoher Ekmanzahl und großem Radienverhältnis}

Alle drei von Kida \& Kitauchi (1998) präsentierten Dynamos zeigen Umkehrungen des Dipolfeldes, und zwar in regelmäßiger, nahezu regelmäßiger bzw. chaotischer Weise, abhängig vom Wert der Robertszahl. Ihr Modell ist dem hier benutzten sehr ähnlich: Boussinesq, ohne leitfähigen inneren Kern, feste Ränder, und durch einen vorgegebenen Temperaturkontrast angetriebene Konvektion. Das Radienverhältnis von innerem zu äußerem Kern beträgt bei ihnen allerdings $r_{q}=r_{i} / r_{0}=0.5$, die Dicke ihrer Kugelschale ist also wesentlich geringer.

Um ihre Lösungen zu reproduzieren, wird $r_{q}=0.5$ gewählt und dann ähnlich vorgegangen, wie in Kida \& Kitauchi (1998) berichtet: Zuerst wird eine reine Konvektions-Simulation gestartet, indem bei $\operatorname{Pr}=1, \mathrm{E}=2.2 \times 10^{-2}$ und 1.7-fach überkritischer Rayleighzahl dem konduktiven Temperaturprofil eine kleine zufällige Störung überlagert wird. Nach drei viskosen Diffusionszeiten stellt sich genau wie bei Kida \& Kitauchi ein quasi-stationärer Zustand ein, der aus fünf in $z$-Richtung ausgedehnten Konvektionszellen besteht, welche symmetrisch zur Äquatorebene sind. Die Strömungsstruktur gibt Abb. 3.1 wieder, in der die Flüssigkeitsgeschwindigkeit in der Äquatorebene sowie ein Stück unterhalb des äußeren Randes gezeigt ist. Die kinetische Energie der reproduzierten Lösung ist zwar 8\% kleiner, insgesamt ergibt sich aber eine gute Übereinstimmung. Kritische Rayleighzahlen für festes $\Delta T$ (Typ 1) für $r_{q}=0.5$ wurden für die Ekmanzahlen $\mathrm{E}_{1}=2.2 \times 10^{-2}, \mathrm{E}_{2}=10^{-2}$ und $\mathrm{E}_{3}=10^{-3}$ ermittelt $\mathrm{zu} \mathrm{Ra}_{\text {krit } 1}=80, \mathrm{Ra}_{\text {krit2 }}=59$ und Rakrit3 $=44$ mit einer Genauigkeit von $\pm 1 \%$. Die Bestimmung der Ra $a_{\text {krit }}$ erfolgt wie in Kap. 4.2.1 von Kutzner (1999) beschrieben.

Auf die so erhaltene Lösung wird bei $\mathrm{q}=10$ in einer ca. 1 viskose Diffusionszeit langen Phase der Magnetokonvektion ein toroidales, axialsymmetrisches Magnetfeld (positives $B_{\phi}$ in der Nordhemisphäre, negatives in der Südhemisphäre) aufgeprägt und dann wieder abgeschaltet. Es stellt sich ein periodisch oszillierender, selbsterhaltender Dynamo ein, der alle 2.8 magnetische Diffusionszeiten seine Polarität wechselt. Die Strömungsstruktur im Fall mit Magnetfeld bleibt im wesentlichen dieselbe wie ohne Magnetfeld, lediglich die kinetische Energie sinkt von 18.5 im nichtmagnetischen Fall auf 16.3 bei $\mathrm{q}=10 \mathrm{ab}$. Ein Ausschnitt 


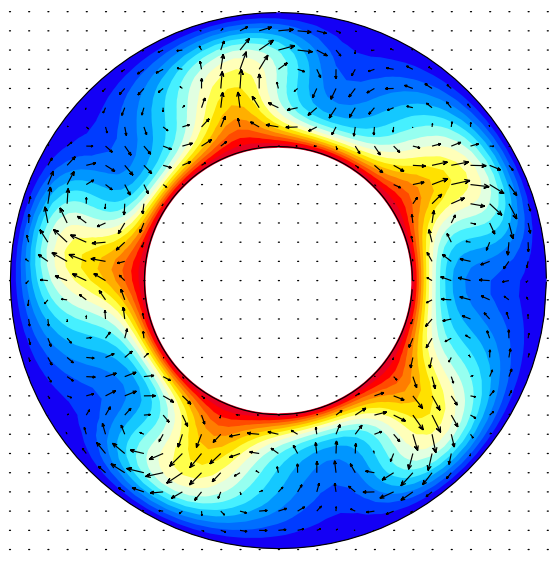

(a) Geschwindigkeitspfeile und Temperatur in der Äquatorebene, Rottöne entsprechen hoher, Blautöne niedriger Temperatur.

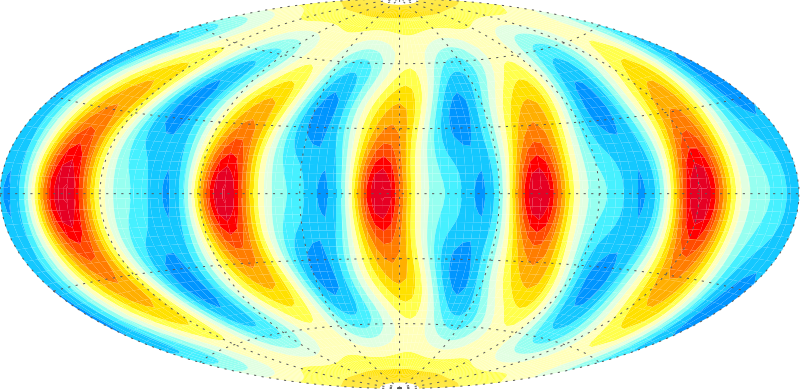

(b) Radialgeschwindigkeit $v_{r}$ bei $r=0.93 r_{0}$. Rot positives $v_{r}$ (Aufstrom), blau negatives $v_{r}$ (Abstrom).

Abb. 3.1: Strömungsstruktur bei festem $\Delta T, r_{q}=0.5, \mathrm{E}=2.2 \times 10^{-2}, \mathrm{Ra}=1.7 \times \mathrm{Ra}_{\text {krit }}$.

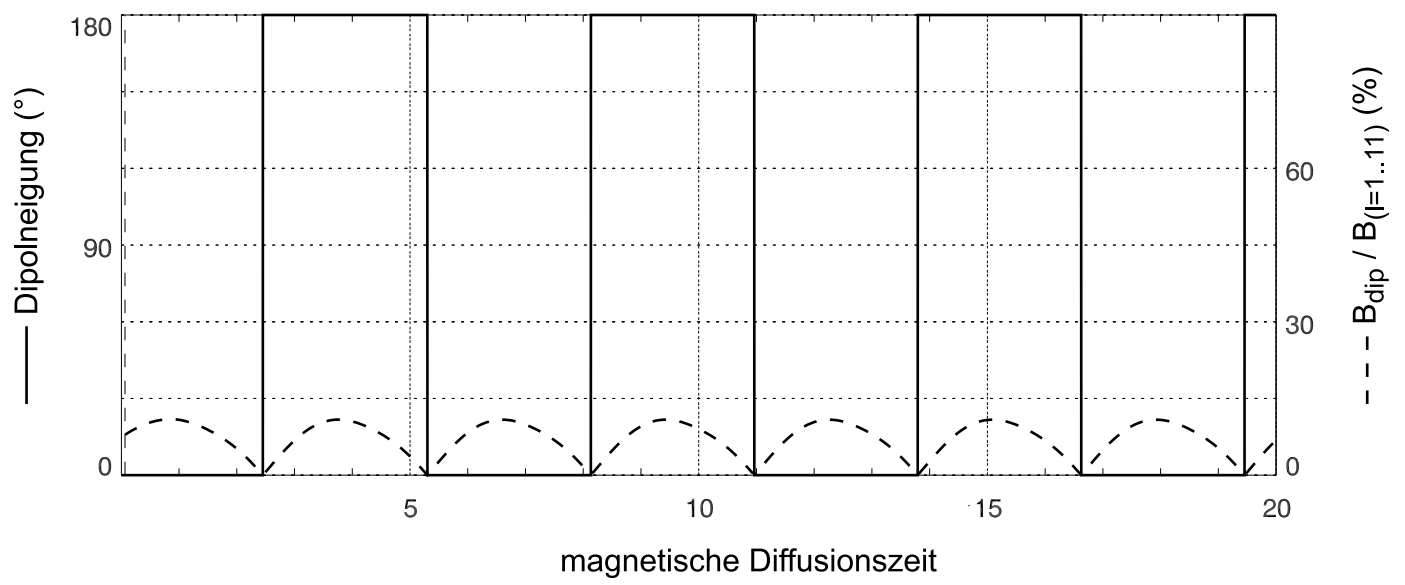

Abb. 3.2: Zeitreihe des periodisch umkehrenden Dynamos, festes $\Delta T, r_{q}=0.5, \mathrm{E}=2.2 \times$ $10^{-2}, \mathrm{Ra}=1.7 \times \mathrm{Ra}_{\text {krit }}$. Linke Skala, durchgezogene Linie: Dipolneigung; rechte Skala, gestrichelte Linie: relativer Dipolbeitrag zum KMG-Feld in Prozent (i. e. die rms-Stärke des Dipolfeldes geteilt durch die rms-Feldstärke in den Moden $\ell=$ $1 \ldots 11)$. 


\begin{tabular}{crrrrrl}
\hline $\mathrm{q}$ & $\mathrm{Rm}$ & $\mathrm{Nu}$ & $\bar{E}_{\mathrm{mag}}$ & $\bar{E}_{\mathrm{mag}}^{\ell=1}$ & $\bar{B}_{\mathrm{KMG}}$ & $\bar{B}_{\text {dip }}$ \\
\hline 8.3 & 50 & 1.35 & - & - & - & - \\
10 & 57 & 1.33 & 5.0 & $0.4 \%$ & 0.55 & 0.04 \\
14.3 & 77 & 1.30 & 7.3 & $6.1 \%$ & 0.77 & 0.24 \\
\hline
\end{tabular}

Tab. 3.1: Daten für Modelltyp 1 (festes $\Delta T$ ) bei $r_{q}=0.5, \mathrm{E}=2.2 \times 10^{-2}, \mathrm{Ra}=135.3=$ $1.7 \times \mathrm{Ra}_{\text {krit }}$. Für $\mathrm{q}=8.3$ zerfiel das Magnetfeld mit der Zeit.

der insgesamt 60 magnetische Diffusionszeiten langen Zeitreihe dieses Dynamos ist in Abb. 3.2 zu finden.

Die Umkehrungen finden streng periodisch statt, allerdings erreicht der relative Dipolbeitrag an der KMG durchschnittlich nur 7\%. Während der Umpolung sinkt er auf Null, d. h. es gibt zu diesem Zeitpunkt weder einen axialen noch einen äquatorialen Dipol. Einige Kennzahlen dieses Dynamos sowie zweier anderer Simulationen, die ausgehend von der Lösung bei $\mathrm{q}=10$ mit höherer bzw. niedrigerer Robertszahl gestartet wurden, sind in Tab. 3.1 zusammengestellt.

Abb. 3.3 gibt eine Vorstellung davon, wie diese periodischen Umpolungen ablaufen. Zu sehen sind Schnappschüsse des radialen Magnetfeldes $B_{r}$ an der äußeren Grenze in Zeitabständen von 0.5 magnetischen Diffusionszeiten; die Zeit ist mit Abb. 3.2 konsistent. Da $B_{r}$ hier antisymmetrisch zur Äquatorebene ist, ist nur die nördliche Hemisphäre gezeigt. Wo im Norden Feld in die Kugelschale eindringt, tritt es bei gleicher Länge und Breite im Süden wieder aus. Die fünffache Symmetrie des Geschwindigkeitsfeldes in der Länge $\phi$ wirkt sich auch auf das Magnetfeld aus, in dem an (maximal) fünf Positionen starke Flußbündel zu finden sind. Das ist z. B. im Schnappschuß bei $t=2.0$ gut zu erkennen. Die starken Flußkonzentrationen liegen immer in der Nähe von Abströmen; man kann also annehmen, daß die Feldlinien im Sinne des frozen-flux Theorems (siehe z. B. Jacobs (1994)) von den Abströmen eingesammelt und gebündelt werden.

Im Grenzfall verschwindender magnetischer Diffusion (bzw. unendlicher elektrischer Leitfähigkeit) wird eine Feldlinie, die zu einem bestimmten Zeitpunkt durch ein Flüssigkeitspaket verläuft, auch zu jedem anderen Zeitpunkt durch dieses Flüssigkeitspaket verlaufen. Man sagt, die Feldlinie sei in der Flüssigkeit ,eingefroren“. Auch für Fälle, in denen magnetische Diffusion zwar noch vorhanden, aber sehr gering ist, ist das Konzept der eingefrorenen Feldlinien eine hilfreiche Vorstellung. Die magnetische Reynoldszahl beträgt hier $\mathrm{Rm}=57$, d. h. also, daß der Advektionsterm über fünfzigmal größer als der Diffusionsterm ist und Magnetfeld effektiv vom Geschwindigkeitsfeld mitbewegt wird.

Während der sechs Diffusionszeiten wechselt der Dynamo zweimal seine Polarität, die sich dadurch definiert, ob von den fünf maximal vorhandenen Flußbündelungen mehr die normale oder die inverse Polarität aufweisen. Betrachten wir den Ablauf einer Umkehrung. Wir starten bei $t=0.0$ mit zwei Konzentrationen nach außen gerichteten Flusses (definieren wir sie als normale Polarität) an den Positionen 1 und 4, und dazwischen einer Konzentration nach innen gerichteten Flusses (invers, Position 5). Bei $t=1.0$ bildet sich an Pos. 2-3, an der bisher keine Bündel vorhanden waren, jetzt ein Paar mit normalem und inversem Fluß, welches bis $t=2.0$ in seiner Stärke anwächst. Während sich sonst in der Länge immer normale mit inversen Flußbündeln abwechseln, entstehen an Pos. 1-2 so zwei Bündel gleicher Pola- 


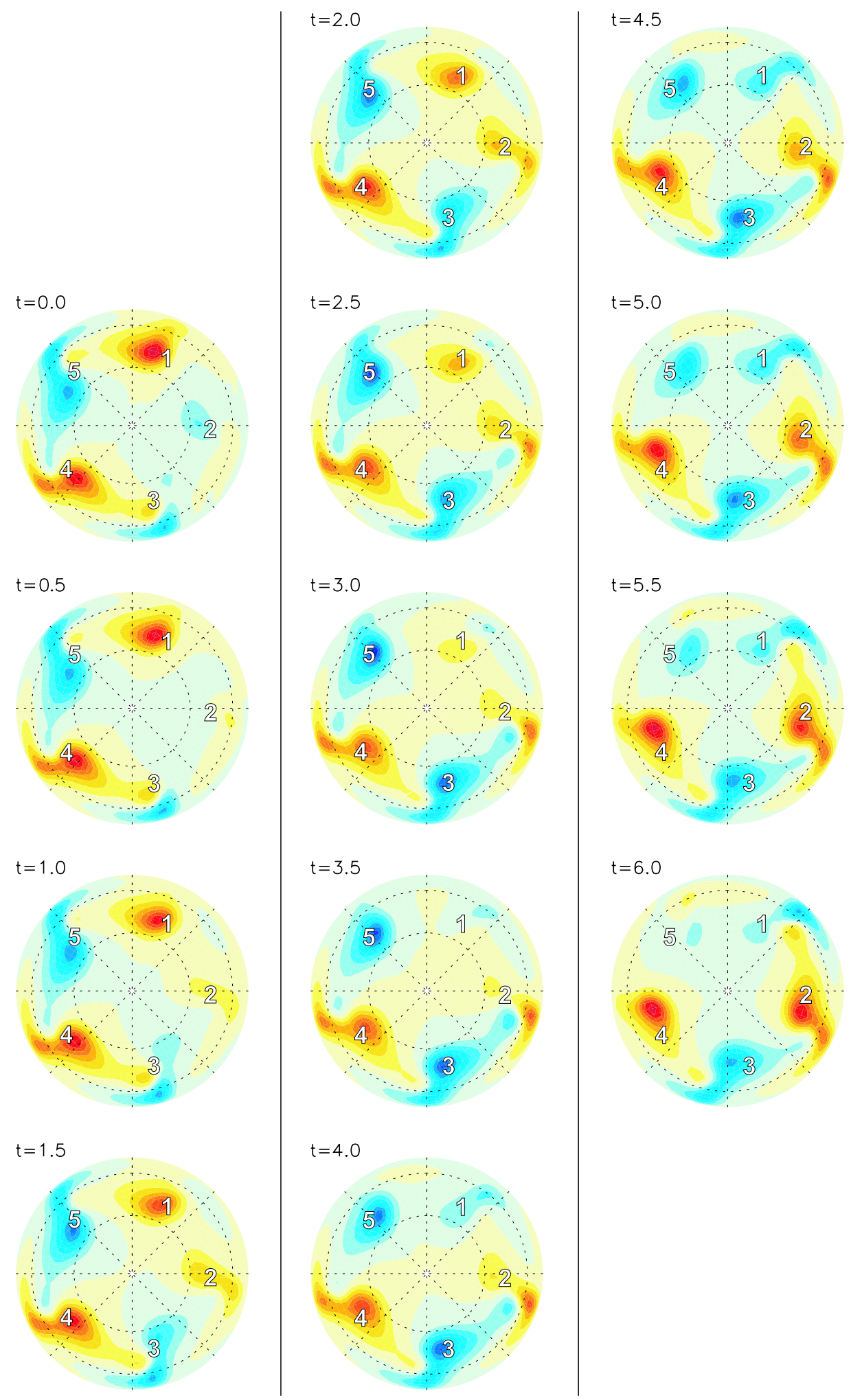

Abb. 3.3: Radiales Magnetfeld $B_{r}$ des Dynamos aus Bild 3.2 Rottöne positives, Blautöne negatives $B_{r}$. Ein Konturschritt entspricht einem $\Delta B_{r}$ von 0.26 . Blick von Norden auf die äußere Kugelschale (KMG). 
rität. Ab $t=2.5$ werden diese schwächer, bis sie bei $t=3.0$ fast ganz verschwunden sind und dadurch die Polarität auf invers gewechselt hat. $\mathrm{Ab} t=3.5$ wird an Pos. $2 \mathrm{u} .1$ ein Paar normalen und inversen Flusses aufgebaut, wodurch an Pos. 1 u. 5 jetzt zwei Bündel inversen Flusses nebeneinander liegen, welche bis $t=5.0$ vorherrschen, sich dann aber abschwächen, wodurch die Polarität kurz vor $t=5.5$ wieder zu normal wechselt. Bei $t=6.0$ ist die Situation wieder wie am Anfang (zwei normale und ein inverses Bündel), nur daß die „Leerstelle“, also die beiden Positionen ohne starke Flußbündel, von Pos. 3 u. 2 weiter gerückt ist auf die Pos. 1 u. 5.

Zusätzlich zu dem in der Abbildung gezeigten zeitlichen Ablauf driften die Flußbündel noch sehr schnell (631 ${ }^{\circ}$ pro magnetische Diffusionszeit) in retrograde (westliche) Richtung, doch diese Drift ist für die Darstellung abgezogen worden, damit man die wesentlichen Vorgänge sehen kann.

Es stellt sich die Frage, warum dieser Dynamo in der Weise umpolt, wie wir es hier sehen. Man weiß zwar heutzutage, welche Mechanismen in einem Dynamo ablaufen, die ihn am Leben erhalten (Olson et al., 1999; Kitauchi \& Kida, 1998), die Funktionsweise von Polumkehrungen ist aber noch ungeklärt und Gegenstand aktueller Forschung. Einige Hinweise sollen hier aber doch gesammelt werden, z. B. ob die ungerade Anzahl an Konvektionszellen bzw. sogar die Anzahl Fünf für diese Umkehrungen wichtig ist.

Fünf ist die natürliche Zahl an Konvektionszellen, die sich bei dieser Ekmanzahl nahe der kritischen Rayleighzahl einstellt. Statt alle Moden zu initialisieren, kann man die Konvektion auch starten, indem nur eine bestimmte Mode des Temperaturfeldes angeregt wird. Das wird mit den Moden $l=m=4, l=m=6$ und $l=m=7$ gemacht, eine konvektierende Lösung mit 5-facher Längensymmetrie existiert ja bereits. Auf die konvektierenden Lösungen mit 4, 5, 6 bzw. 7-facher Symmetrie in $\phi$ wird dann wie oben für kurze Zeit ein Magnetfeld aufgeprägt und dann wieder abgeschaltet, um zu sehen, ob sich ein selbsterhaltender Dynamo einstellt. Das wird bei $\mathrm{q}=8.3,10,14.3$ und 20 gemacht.

Bei 4- und 6-facher Längensymmetrie zerfällt das Magnetfeld in allen Fällen bis auf den Fall $q=20$ bei 6-facher Symmetrie. Dort ergibt sich eine nicht-umpolende Lösung, welche aus sechs Flußkonzentrationen normaler Polarität bei etwa $50^{\circ}$ Breite und sechs schwächeren, inversen Konzentrationen bei etwa $20^{\circ}$ Breite besteht (antisymmetrisch zum Äquator), s. Abb. 3.4. Diese Struktur findet sich auch bei allen Lösungen mit 4- bzw. 6-facher Symmetrie (mit entsprechender Anzahl an Flußbündeln), nur daß der Dynamo unterkritisch ist in dem Sinne, daß die Magnetfelder sich nicht selbst erhalten und deshalb langsam zerfallen.

Ein wichtiger Unterschied des periodisch umkehrenden Dynamos zu den anderen gefundenen ist, daß die Flußbündel bei den Breiten von $50^{\circ}$ und $20^{\circ}$ die gleiche Polarität aufweisen und deswegen zu einem größeren zusammengewachsen sind, wobei die Polarität der Flußbündel entlang $\phi$ wechseln kann (Abb. 3.4 oben). Die Verknüpfung nebeneinander liegender Flußbündel entgegengesetzter Polarität ist stark, was an dem horizontalen Feld (Vektorpfeile) sichtbar wird. Z. B. tritt Feld bei $-180^{\circ}$ Länge und $-50^{\circ}$ Breite ins Innere der Kugelschale, tritt bei gleicher Länge auf der Nordhalbkugel wieder aus, tritt weiter im Südosten in das benachbarte Flußbündel bei $-100^{\circ}$ Breite wieder ein, um dann wieder in der Südhemisphäre bei gleicher Länge auszutreten und die Schleife zu schließen. Dieser Feldlinienverlauf ist nicht möglich, wenn in $\phi$ zwei Flußbündel gleicher Polarität nebeneinander liegen (wie im unteren Bild der Abbildung). Genau an diesen Stellen ist die Magnetfeldkonfiguration instabil (vgl. dazu Abb. 3.3), was in der Tat eine ungerade Anzahl an Konvektionszellen erfordert. 

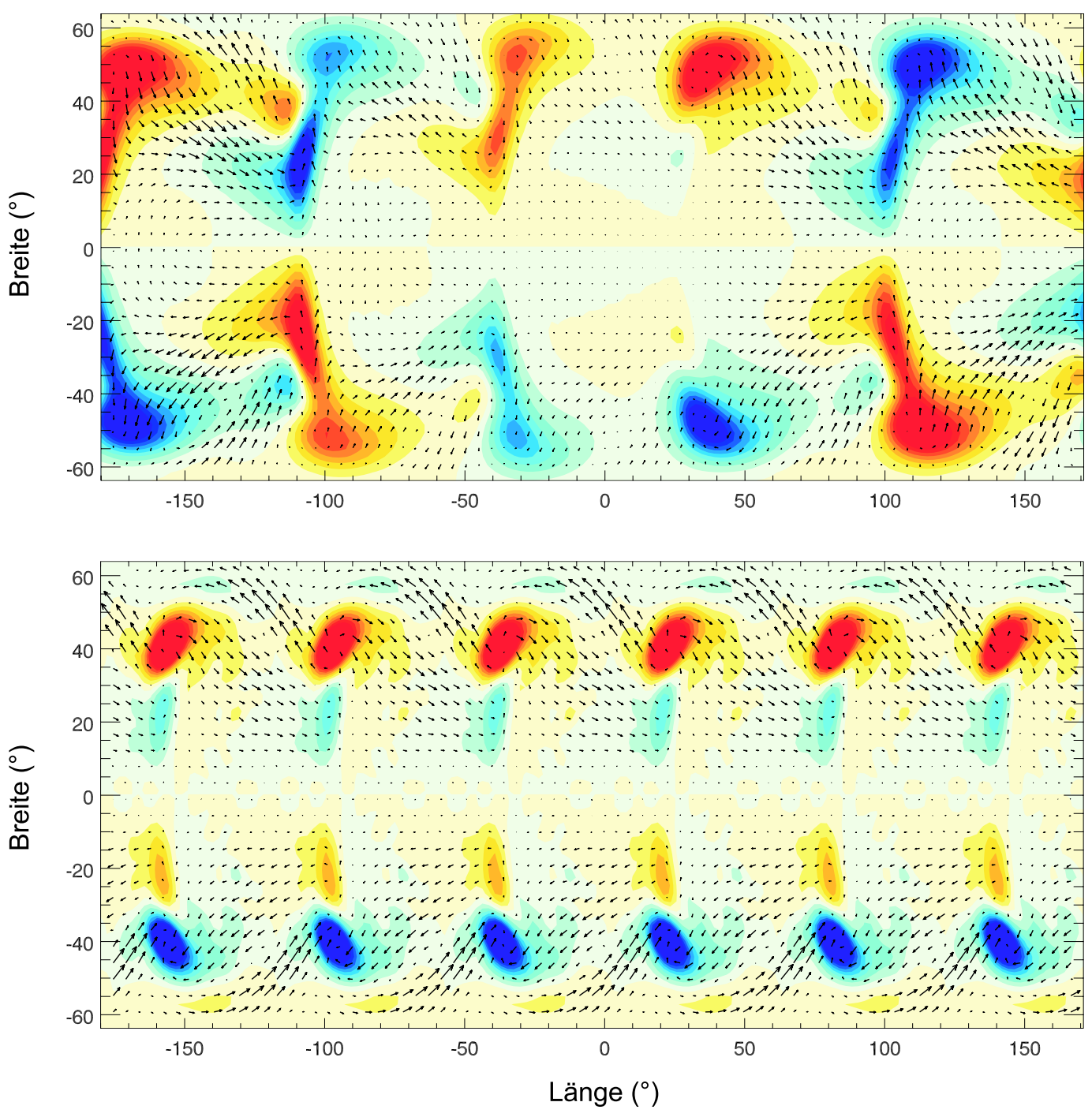

Abb. 3.4: Schnappschüsse des Magnetfeldes bei $r=0.96 r_{0}$. Rot positives, blau negatives $B_{r}$, Vektorpfeile kennzeichnen horizontales Magnetfeld. Oben: Periodisch oszillierender Dynamo mit 5 Konvektionszellen, $\mathrm{q}=10$. Unten: Stabiles, dipol-dominiertes Feld im Fall mit 6 Konvektionszellen und $\mathrm{q}=20$. 
Die Lösung, die mit 7-zähliger Symmetrie der Konvektion gestartet wurde, geht nach kurzer Zeit in eine Lösung mit 5-zähliger Symmetrie über. Bei $q=20$ sowie $q=14.3$ treten dann chaotische Umkehrungen des Feldes auf, welches einen durchschnittlichen Dipolbeitrag von $25 \%(\mathrm{q}=14.3)$ bzw. 17\% $(\mathrm{q}=20)$ relativ zum Gesamtfeld an der KMG hat.

Das Verhalten des Dynamos bei $\mathrm{q}=10$ ist also ein Spezialfall, da periodische Umkehrungen in dieser Weise sonst nicht gefunden werden konnten. Die chaotisch umkehrenden Lösungen bei höherer Robertszahl sind allerdings aufgrund ihres signifikanten Dipolbeitrags interessant, und es soll versucht werden, ähnliches Verhalten auch für das dem Erdkern entsprechenden Radienverhältnis von $r_{q}=0.35$ zu bekommen (Kap. 3.2).

\subsection{Parameterabhängigkeit der Dynamoregimes}

Der Parameterraum wird am umfangreichsten erforscht für Dynamos mit einem vorgegebenen Temperaturkontrast als Antrieb der Konvektion (Modell Typ 1). Dies geschieht, indem alle dimensionslosen Eingabeparameter E, q, Ra außer der Prandtlzahl systematisch variiert werden. In Tab. 3.2 werden die kritischen Rayleighzahlen für einige der Modelltypen aufgelistet. Eine Zusammenstellung der charakteristischen Daten der Simulationen wird in Tab. 3.3 gegeben; für einige Bilder der Kap. 3.2 und 3.3 werden auch Daten aus Christensen et al. (1999) und Kutzner \& Christensen (2000) benutzt.

Bei allen fünf untersuchten Werten der Ekmanzahl zeigen sich zwei unterschiedliche Dynamoregimes, welche durch eine überraschend klare Grenze voneinander getrennt sind. Bild 3.5 zeigt die zeitlich gemittelten rms-Werte des Magnetfeldes an der äußeren Grenze der Modelle (der KMG). Bei kleiner Ra oder q können keine selbsterhaltenden Dynamos gefunden werden. Die magnetische Energie zerfällt exponentiell (graue Gebiete, Simulationen mit zerfallenden Magnetfeldern sind durch Sterne gekennzeichnet). Für moderate Ra erhält man Magnetfelder mit starken Dipolbeiträgen. Nahe des Einsatzes selbserhaltender Dynamowirkung, also bei kleinen Ra und kleinen q im weißen Bereich, trägt der Dipol zu mehr als einem Drittel der gesamten magnetischen Energie innerhalb der Kugelschale bei. An der KMG entfällt mehr als die Hälfte der rms-Feldstärke allein auf das Dipolfeld. Der Neigungswinkel der Dipolachse beträgt durchschnittlich wenige Grad und er überschreitet nur ganz selten und für kurze Zeit die $10^{\circ}$ Marke.

Diese Dipoldominanz verschwindet bei einer bestimmten Rayleighzahl Ra $\mathrm{R}_{\mathrm{R}}$, welche durch

\begin{tabular}{rcccccc}
\hline & & \multicolumn{5}{c}{ Ekmanzahl } \\
Antriebsart & $2 \times 10^{-2}$ & $10^{-2}$ & $3 \times 10^{-3}$ & $10^{-3}$ & $3 \times 10^{-4}$ & $10^{-4}$ \\
\hline Festes $\Delta T(1)$ & 105,6 & 83,0 & 59,0 & 55,9 & 60,8 & 69,7 \\
$100 \%$ interne Heizung (2) & & & & 52,2 & 67,8 & 91,9 \\
$50 \%$ interne Heizung (3) & & & & 52,9 & 61,1 & 73,9 \\
chemische Konvektion (8) & & & & 53,2 & 56,1 & 62,2 \\
\hline
\end{tabular}

Tab. 3.2: Kritische Rayleighzahlen Rakrit für einige Modelltypen, Genauigkeit: $\pm 0.5 \%$. Radienverhältnis $r_{q}=0.3505747$. (Die Rakrit für $\mathrm{E}=10^{-3}$ bis $10^{-4}$ für die Antriebstypen 1, 2 und 8 sind aus Kutzner (1999) entnommen.) 


\begin{tabular}{ccrrrrrrcc}
\hline $\mathrm{E}$ & $\mathrm{q}$ & $\mathrm{Ra}$ & $\mathrm{Rm}$ & $\mathrm{Nu}$ & $\bar{E}_{\mathrm{mag}}$ & $\bar{E}_{\mathrm{mag}}^{\ell=1}$ & $\bar{B}_{\mathrm{KMG}}$ & $\bar{B}_{\text {dip }}$ & Neigung [ $\left.^{\circ}\right]$ \\
\hline $2 \times 10^{-2}$ & 10 & 200 & 59 & 1.30 & 10 & $11.7 \%$ & 0.65 & 0.31 & $0.0(0.0)$ \\
$2 \times 10^{-2}$ & 10 & 250 & 72 & 1.35 & 14 & $10.7 \%$ & 0.73 & 0.34 & $176.5(1.9)$ \\
$2 \times 10^{-2}$ & 10 & 300 & 94 & 1.48 & 9 & $7.9 \%$ & 0.46 & 0.19 & $\mathrm{r}$ \\
$2 \times 10^{-2}$ & 10 & 400 & 123 & 1.65 & 12 & $6.0 \%$ & 0.48 & 0.15 & $\mathrm{r}$ \\
$2 \times 10^{-2}$ & 10 & 600 & 170 & 1.88 & 22 & $4.9 \%$ & 0.60 & 0.15 & $\mathrm{r}$ \\
$2 \times 10^{-2}$ & 10 & 800 & 204 & 2.04 & 38 & $4.7 \%$ & 0.83 & 0.18 & $\mathrm{r}$ \\
$2 \times 10^{-2}$ & 15 & 300 & 137 & 1.48 & 16 & $5.9 \%$ & 0.73 & 0.25 & $\mathrm{r}$ \\
$3 \times 10^{-3}$ & 6 & 300 & 148 & 1.91 & $*$ & & & & $\mathrm{r}$ \\
$3 \times 10^{-3}$ & 10 & 120 & 70 & 1.36 & 380 & $31.0 \%$ & 2.33 & 1.47 & $2.6(0.1)$ \\
$3 \times 10^{-3}$ & 10 & 210 & 128 & 1.55 & 325 & $14.9 \%$ & 1.52 & 0.87 & $5.4(3.3)$ \\
$3 \times 10^{-3}$ & 10 & 300 & 248 & 1.92 & 63 & $5.2 \%$ & 0.40 & 0.11 & $\mathrm{r}$ \\
$10^{-3}$ & 6 & 300 & 160 & 2.06 & 1020 & $12.0 \%$ & 1.18 & 0.60 & $6.9(3.7)$ \\
$10^{-3}$ & 10 & 200 & 160 & 1.60 & 1260 & $16.1 \%$ & 1.72 & 1.01 & $0.0(0.0)$ \\
$10^{-3}$ & 10 & 600 & 508 & 2.89 & 950 & $2.0 \%$ & 1.11 & 0.18 & $\mathrm{r}$ \\
$3 \times 10^{-4}$ & 2 & 990 & 255 & 4.01 & $\approx 10$ & $1.4 \%$ & 0.02 & 0.002 & $\mathrm{r}$ \\
$3 \times 10^{-4}$ & 2 & 1350 & 314 & 4.74 & 670 & $1.1 \%$ & 0.19 & 0.02 & $\mathrm{r}$ \\
$3 \times 10^{-4}$ & 5 & 380 & 195 & 2.33 & 460 & $13.0 \%$ & 1.18 & 0.70 & $4.8(2.7)$ \\
$3 \times 10^{-4}$ & 6 & 810 & 495 & 3.68 & 4900 & $4.0 \%$ & 1.31 & 0.44 & $5.4(2.7)$ \\
$3 \times 10^{-4}$ & 3 & 380 & 117 & 2.23 & 3350 & $20.0 \%$ & 0.78 & 0.57 & $4.0(2.3)$ \\
$3 \times 10^{-4}$ & 3 & 630 & 199 & 3.10 & 3850 & $12.0 \%$ & 0.89 & 0.50 & $3.5(1.9)$ \\
$3 \times 10^{-4}$ & 3 & 810 & 308 & 3.75 & 390 & $1.3 \%$ & 0.17 & 0.03 & $\mathrm{e}$ \\
$3 \times 10^{-4}$ & 3 & 990 & 350 & 4.25 & 1050 & $1.5 \%$ & 0.31 & 0.06 & $\mathrm{r}$ \\
$3 \times 10^{-4}$ & 3 & 1350 & 437 & 4.77 & 1750 & $0.9 \%$ & 0.40 & 0.03 & $\mathrm{r}$ \\
$3 \times 10^{-4}$ & 3 & 2130 & 592 & 5.85 & 3800 & $0.8 \%$ & 0.63 & 0.04 & $\mathrm{r}$ \\
$10^{-4}$ & 0.67 & 1500 & 158 & 5.88 & 1300 & $2.8 \%$ & 0.09 & 0.02 & $\mathrm{r}$ \\
$10^{-4}$ & 0.67 & 2000 & 194 & 7.04 & 2800 & $2.2 \%$ & 0.14 & 0.02 & $\mathrm{r}$ \\
$10^{-4}$ & 0.67 & 2500 & 221 & 7.32 & 8000 & $2.6 \%$ & 0.24 & 0.04 & $\mathrm{r}$ \\
$10^{-4}$ & 1 & 2000 & 273 & 6.51 & 6400 & $1.4 \%$ & 0.28 & 0.04 & $\mathrm{H}$ \\
$10^{-4}$ & 2 & 1050 & 229 & 4.20 & 22000 & $15.0 \%$ & 1.01 & 0.66 & $3.1(1.7)$ \\
$10^{-4}$ & 2 & 1750 & 465 & 6.04 & 7600 & $0.9 \%$ & 0.44 & 0.04 & $\mathrm{r}$ \\
\hline & & & & & & & & & \\
\end{tabular}

Tab. 3.3: Ergebnisse der Parameterstudie für Modelltyp 1 (festes $\Delta T$ ). Neigung: durchschnittliche Neigung der Dipolachse (in Klammern: Standardabweichung) e: Exkursionen (Dipolneigung wird zeitweise $>30^{\circ}$ ), r: Umkehrungen der Dipolachse, $\mathrm{H}$ : hemisphärischer Dynamo. (*: $\bar{E}_{\mathrm{mag}}$ variiert zwischen $10^{-6}$ und 100 . Die Dipolneigung ist entweder $0^{\circ}$ oder $180^{\circ}$.) 


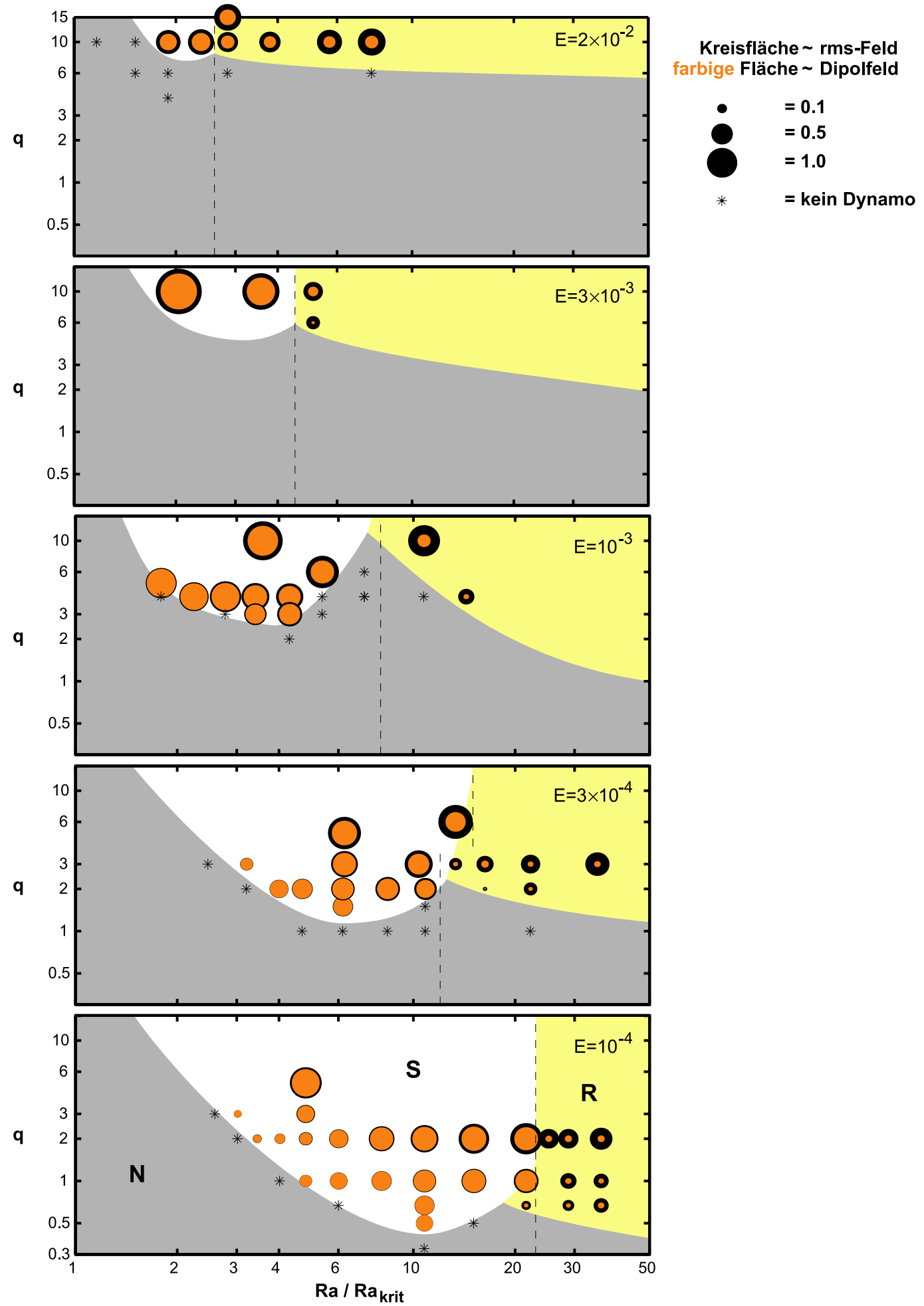

Abb. 3.5: Zeitgemittelte Magnetfelder an der KMG für Modelltyp 1 (festes $\Delta T$ ) bei $\mathrm{E}=$ $2 \times 10^{-2}, 3 \times 10^{-3}, 10^{-3}, 3 \times 10^{-4}$ und $10^{-4}$. Gezeigt sind die rms-Felder als Funktion der Rayleighzahl (normiert mit ihrem kritischen Wert) und der Robertszahl. Die Kreisfläche zeigt das Gesamtfeld, die farbige innere Fläche das Dipolfeld. Keine selbsterhaltenden Dynamos in den grauen (N) Gebieten (Sterne). Weiße Gebiete (S): Regime mit stabilen, dipol-dominierten Dynamos; Gelbe Gebiete $(\mathbf{R})$ : Dynamos mit kleinen Dipolbeiträgen und umkehrenden Polaritäten. 
die gestrichelte vertikale Linie angedeutet wird. Bei Rayleighzahlen, die höher als Ra $a_{R}$ sind, fällt die Energie des Dipolanteils für Ekmanzahlen $\mathrm{E} \leq 10^{-3}$ typischerweise unter 3\% der gesamten magnetischen Energie in der Kugelschale. Bei $\mathrm{E}=2 \times 10^{-2} \mathrm{kann}$ sie allerdings bis $8 \%$ erreichen. An der KMG trägt das Dipolfeld durchschnittlich noch $10 \%$ zum gesamten rms-Feld bei, dieser Anteil ist zeitlich jedoch sehr variabel. Die Neigungswinkel der Dipolachsen liegen zwischen $0^{\circ}$ und $180^{\circ}$, jedoch bevorzugt in hohen Breiten. Spezielle Fälle mit kleinem Dipolbeitrag stellen die hemisphärischen Dynamos dar, die in Grote et al. (2000b) beschrieben werden. Bei ihnen operiert der Dynamo nur in der nördlichen oder südlichen Hemisphäre, während die jeweils andere nahezu feldfrei ist. Im Rahmen dieser Parameterstudie wurde auch solch ein Dynamo gefunden; er ist in Tab. 3.3 mit einem " $\mathrm{H}$ " gekennzeichnet.

Die Grenze zwischen den beiden Regimes hängt in keiner klar erkennbaren Weise von der Robertszahl q ab. Das Diagramm für $\mathrm{E}=3 \times 10^{-4}$ gibt einen leichten Hinweis darauf, daß das dipolare Regime bei großen q bevorzugt wird: Bei 13-fach überkritischer Ra befindet sich der Dynamo bei $\mathrm{q}=3$ schon im umkehrenden Regime, während er bei $\mathrm{q}=6$ stabil und dipol-dominiert ist. Für eine klare Aussage müßte die Regimegrenze allerdings weiter zu hohen q verfolgt werden, was aber hinsichtlich der extrem langen Rechenzeiten unterlassen wurde. Aufgrund der geringen magnetischen Diffusion entstehen bei hohen q sehr kleinskalige Strukturen im Magnetfeld. Diese würden eine weitere Verfeinerung der Gitterauflösung erfordern, welche aber wegen der hohen Rayleighzahl ohnehin schon an der praktischen Grenze ist.

Betrachtet man die Lage der Regimegrenze in Abhängigkeit von Ra, so verschiebt sie sich für fallende E zu höheren überkritischen Ra und zwar mit einer ungefähren Abhängigkeit

$$
\frac{\mathrm{Ra}_{\mathrm{R}}}{\mathrm{Ra}_{\mathrm{krit}}} \simeq 0.1 \cdot \mathrm{E}^{-3 / 5}
$$

im Bereich der Ekmanzahlen $10^{-4} \leq \mathrm{E} \leq 10^{-3}$.

Bild 3.6 zeigt kinetische und magnetische Energien für einen Schnitt durch den Parameterraum bei $\mathrm{E}=10^{-4}$ and $\mathrm{q}=2$. Während die kinetische Energie kontinuierlich mit Ra anwächst, läuft die magnetische Energie im dipolaren Bereich in eine Sättigung und fällt dann an der Regimegrenze signifikant ab, bevor sie im umkehrenden Regime wieder mit Ra anwächst. Jenseits der Regimegrenze ist die kinetische Energie größer als die magnetische, während es im dipolaren Regime umgekehrt ist.

Bei niedriger q können die Regimes durch einen Bereich, in dem es keine selbsterhaltenden Dynamolösungen gibt, voneinander getrennt sein. Bei $\mathrm{E}=10^{-3}$ (mittleres Diagramm in Bild 3.5) und $\mathrm{q}=4$ gibt es selbsterhaltende Dynamos für niedrige und hohe Ra, nicht aber im dazwischenliegenden Bereich mit $\mathrm{Ra} \approx(5-11) \times$ überkritisch. Bei anderen E konnte diese Trennung der Regimes nicht nachgewiesen werden.

Wie in Abschnitt 2.4 erwähnt, wurde untersucht, ob es eine Hysterese gibt, d. h. ob in der Nähe von $\mathrm{Ra}_{\mathrm{R}}$ bei gleichen Parametern (E, Ra, q) beide Lösungsäste koexistieren und es von der Startbedingung abhängt, ob der Dynamo umkehrt oder nicht. Obwohl es manchmal viele magnetische Diffusionszeiten gedauert hat, bis der Dynamo nach Änderung der Parameter seinem neuen Zustand erreicht hat, konnte in keinem Fall eine Hysterese festgestellt werden und die endgültige Lösung hing immer nur von $\mathrm{E}$, Ra und $\mathrm{q}$ ab. 


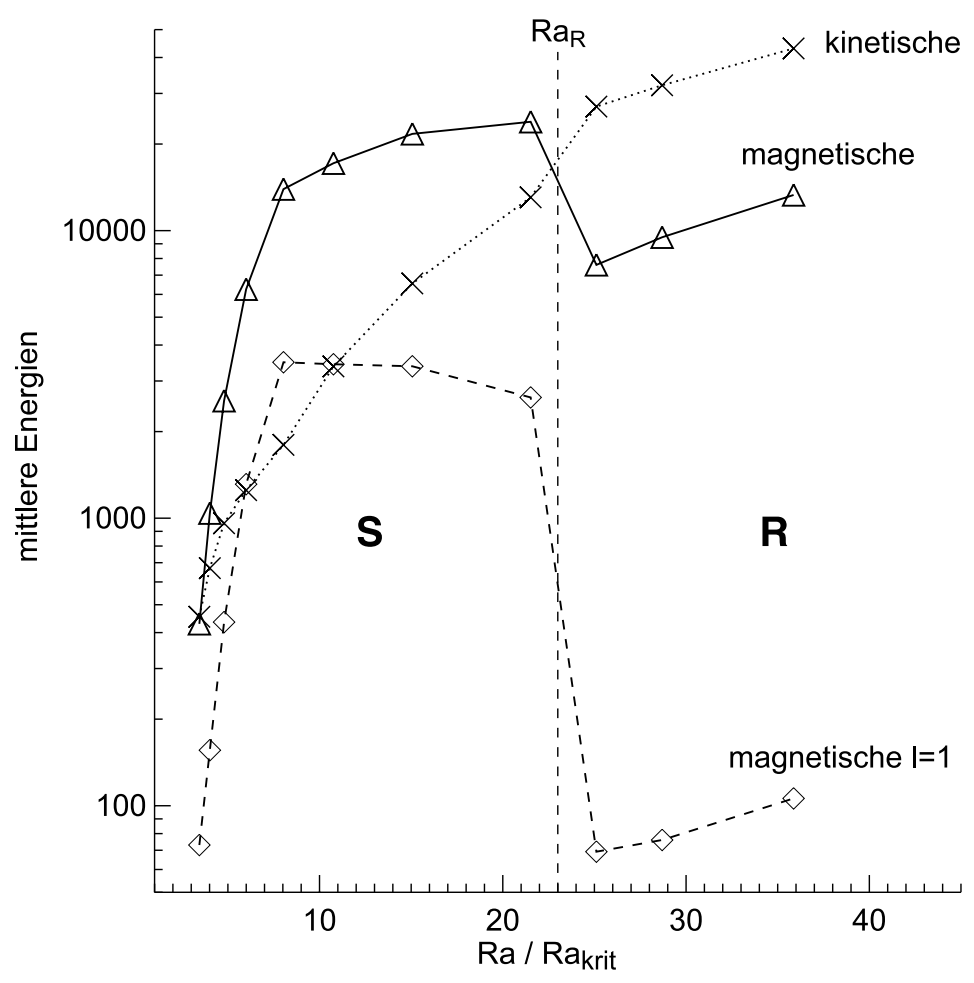

Abb. 3.6: Zeitgemittelte kinetische und magnetische Energiedichten in der Kugelschale als Funktion von $\mathrm{Ra} / \mathrm{Ra}_{\text {krit }}$ für Modelltyp 1 (festes $\Delta T$ ), $\mathrm{E}=10^{-4}, \mathrm{q}=2$. Das Regime stabiler, dipol-dominierter Dynamos (S) für $\mathrm{Ra} / \mathrm{Ra}_{\mathrm{krit}}<\mathrm{Ra}$ ist getrennt vom Regime umkehrender Dynamos mit kleinen Dipolbeiträgen $(\mathbf{R})$.

\subsection{Einfluß der Antriebsart}

Als nächster Schritt werden Dynamos bei fester Ekman- und Robertszahl $\left(E=3 \times 10^{-4}\right.$, $\mathrm{q}=3$ ) für unterschiedliche Antriebsarten (Modelltypen 1-9 aus Abschnitt 2.2) als Funktion der Rayleighzahl betrachtet. Weil die skalierte Differenz von $T$ zwischen innerer und äußerer Grenze kleiner als 1 wird, wenn eine Flußbedingung an mindestens einer der beiden Grenzen benutzt wird, werden die Modelltypen 2-9 als Funktion der effektiven Rayleighzahl $\mathrm{Ra}^{\prime}=$ $\mathrm{Ra} \cdot \mathrm{Nu}$ verglichen. Die Daten für diese Modell-Läufe sind in Tab. 3.4 zu finden.

Bild 3.7zeigt die Intensität des gesamten Magnetfeldes und der Dipolkomponente an der KMG als Funktion von $\mathrm{Ra}^{\prime}$. Der Neigungswinkel der Dipolachse gegen die Rotationsachse beträgt bei den nicht-umkehrenden Modellen wenige Grad, wobei es keine offensichtliche Abhängigkeit des durchschnittlichen Neigungswinkels von der Antriebsart oder von Ra gibt. Für fast alle Antriebsarten findet sich ein Regime mit starken, dipolaren Magnetfeldern von stabiler Polarität $\mathbf{S}$ bei niedrigen Ra und ein Regime mit kleineren Dipolbeiträgen wechselnder Polarität $\mathbf{R}$ bei hohen Ra. Das beweist, daß die Existenz der beiden Dynamoregimes eine robuste Eigenschaft ist, welche nicht von einer bestimmten thermischen Randbedingung abhängt. Lediglich für die größtenteils intern geheizten Modelltypen 2 und 3 gibt es kein dipolares Regime bei diesen Parametern. Selbst die Dynamos nahe der Grenze zum nicht- 


\begin{tabular}{|c|c|c|c|c|c|c|c|c|}
\hline Modell & $\overline{\mathrm{Ra}^{\prime}}$ & $\mathrm{Rm}$ & $\mathrm{Nu}$ & $\bar{E}_{\mathrm{mag}}$ & $\bar{E}_{\mathrm{mag}}^{\ell=1}$ & $\bar{B}_{\mathrm{KMG}}$ & $\bar{B}_{\text {dip }}$ & Neigung $\left[{ }^{\circ}\right]$ \\
\hline (2) & 255 & 215 & 3.36 & 10 & $0.7 \%$ & 0.04 & 0.0001 & $\bar{Q}$ \\
\hline $100 \%$ int. $\mathrm{H}$. & $\dagger 400$ & 331 & 5.26 & 880 & $0.8 \%$ & 0.38 & 0.02 & - \\
\hline (3) & 450 & 212 & 2.80 & 10 & $1.4 \%$ & 0.03 & 0.003 & $\mathrm{r}$ \\
\hline $50 \%$ int. & 660 & 295 & 3.86 & 440 & $1.1 \%$ & 0.21 & 0.02 & $\mathrm{r}$ \\
\hline Heiz. & 1020 & 396 & 5.02 & 1900 & $1.0 \%$ & 0.49 & 0.05 & $\mathrm{r}$ \\
\hline (4) & 227 & $\overline{81}$ & 1.71 & 920 & $23.0 \%$ & 0.37 & 0.31 & - \\
\hline $10 \%$ int. & 390 & 137 & 2.48 & 2650 & $18.0 \%$ & 0.74 & 0.51 & $2.9(1.5)$ \\
\hline \multirow[t]{4}{*}{ Heiz. } & 435 & 151 & 2.67 & 3000 & $17.0 \%$ & 0.81 & 0.53 & $3.3(1.8)$ \\
\hline & 585 & 208 & 3.32 & 2700 & $11.0 \%$ & 0.76 & 0.42 & $3.1(1.8)$ \\
\hline & 879 & 338 & 4.41 & 1000 & $1.3 \%$ & 0.29 & 0.03 & $\mathrm{r}$ \\
\hline & 1386 & 453 & 5.59 & 2750 & $1.0 \%$ & 0.54 & 0.05 & $\mathrm{r}$ \\
\hline$*$ & 882 & 605 & 4.39 & 3400 & $1.1 \%$ & 0.91 & 0.09 & $r$ \\
\hline$(5)$ & 240 & 88 & 1.75 & 860 & $21.0 \%$ & 0.35 & 0.29 & - \\
\hline fester & 363 & 122 & 2.32 & 2750 & $20.0 \%$ & 0.73 & 0.54 & $2.7(1.5)$ \\
\hline Fluß & 461 & 155 & 2.74 & 3200 & $16.0 \%$ & 0.81 & 0.53 & $2.3(1.4)$ \\
\hline \multirow[t]{3}{*}{$\mathrm{IKG}$} & 615 & 218 & 3.42 & 2750 & $11.0 \%$ & 0.76 & 0.42 & $3.3(1.9)$ \\
\hline & 930 & 349 & 4.52 & 1100 & $1.1 \%$ & 0.32 & 0.04 & $\mathrm{r}$ \\
\hline & 1470 & 465 & 5.72 & 3050 & $1.1 \%$ & 0.58 & 0.07 & $\mathrm{r}$ \\
\hline (6) & 236 & 122 & 1.79 & 50 & $8.0 \%$ & 0.06 & 0.04 & $0.0(0.0)$ \\
\hline fester & 378 & 135 & 2.56 & 3700 & $15.0 \%$ & 0.79 & 0.48 & $5.3(2.3)$ \\
\hline Fluß & 421 & 153 & 2.75 & 3850 & $13.0 \%$ & 0.84 & 0.48 & $5.9(2.9)$ \\
\hline KMG & 597 & 217 & 3.52 & 4400 & $10.0 \%$ & 0.95 & 0.46 & $5.3(2.7)$ \\
\hline \multirow[t]{2}{*}{$\& \mathrm{IKG}$} & 900 & 320 & 4.67 & 4600 & $6.0 \%$ & 0.96 & 0.39 & $5.0(2.7)$ \\
\hline & 1397 & 514 & 6.02 & 2900 & $1.3 \%$ & 0.53 & 0.06 & $\mathrm{r}$ \\
\hline (7) & 641 & 205 & 3.28 & 5700 & $9.0 \%$ & 1.06 & 0.51 & $7.0(2.9)$ \\
\hline fester & 972 & 311 & 4.32 & 4800 & $6.0 \%$ & 0.97 & 0.39 & $4.8(2.5)$ \\
\hline Fluß KMG & 1509 & 486 & 5.57 & 3350 & $1.3 \%$ & 0.59 & 0.08 & $\mathrm{r}$ \\
\hline (8) & 233 & 77 & 1.87 & 3250 & $21.0 \%$ & 0.67 & 0.53 & - \\
\hline chem. & 315 & 107 & 2.13 & 3100 & $16.0 \%$ & 0.56 & 0.43 & $4.6(2.4)$ \\
\hline \multirow[t]{7}{*}{ Konv. } & $\dagger 387$ & 122 & 2.38 & 3800 & $15.0 \%$ & 0.68 & 0.48 & $6.6(3.1)$ \\
\hline & $\dagger 573$ & 174 & 2.92 & 4100 & $11.0 \%$ & 0.73 & 0.47 & - \\
\hline & 870 & 258 & 3.86 & 3700 & $7.5 \%$ & 0.66 & 0.37 & $4.0(2.1)$ \\
\hline & 1328 & 383 & 5.05 & 4100 & $3.6 \%$ & 0.60 & 0.25 & e \\
\hline & 1538 & 443 & 5.44 & 3650 & $2.3 \%$ & 0.48 & 0.15 & $10.1(6.1)$ \\
\hline & 1746 & 486 & 5.77 & 3800 & $1.8 \%$ & 0.42 & 0.10 & $\mathrm{r}$ \\
\hline & 2135 & 560 & 6.29 & 4500 & $1.4 \%$ & 0.44 & 0.07 & $\mathrm{r}$ \\
\hline (9) & $\$ 380$ & 128 & 2.44 & 2300 & $15.0 \%$ & 0.49 & 0.37 & - \\
\hline chem. Konv. & 1608 & 512 & 6.24 & 2900 & $1.5 \%$ & 0.34 & 0.05 & $\mathrm{r}$ \\
\hline
\end{tabular}

Tab. 3.4: Ergebnisse der Parameterstudie für die Modelle Typ 2-9 bei $\mathrm{E}=3 \times 10^{-4}, \mathrm{q}=3$ (außer *: q =6). Strich (-): keine Dipolneigung wegen aufgeprägter zweifacher Symmetrie in der Länge, Q: Feld hat quadrupolare Symmetrie. Weitere Erklärungen in 3.3 †on den markierten Simulationen wurde schon in Kutznen (1999) berichtet. 


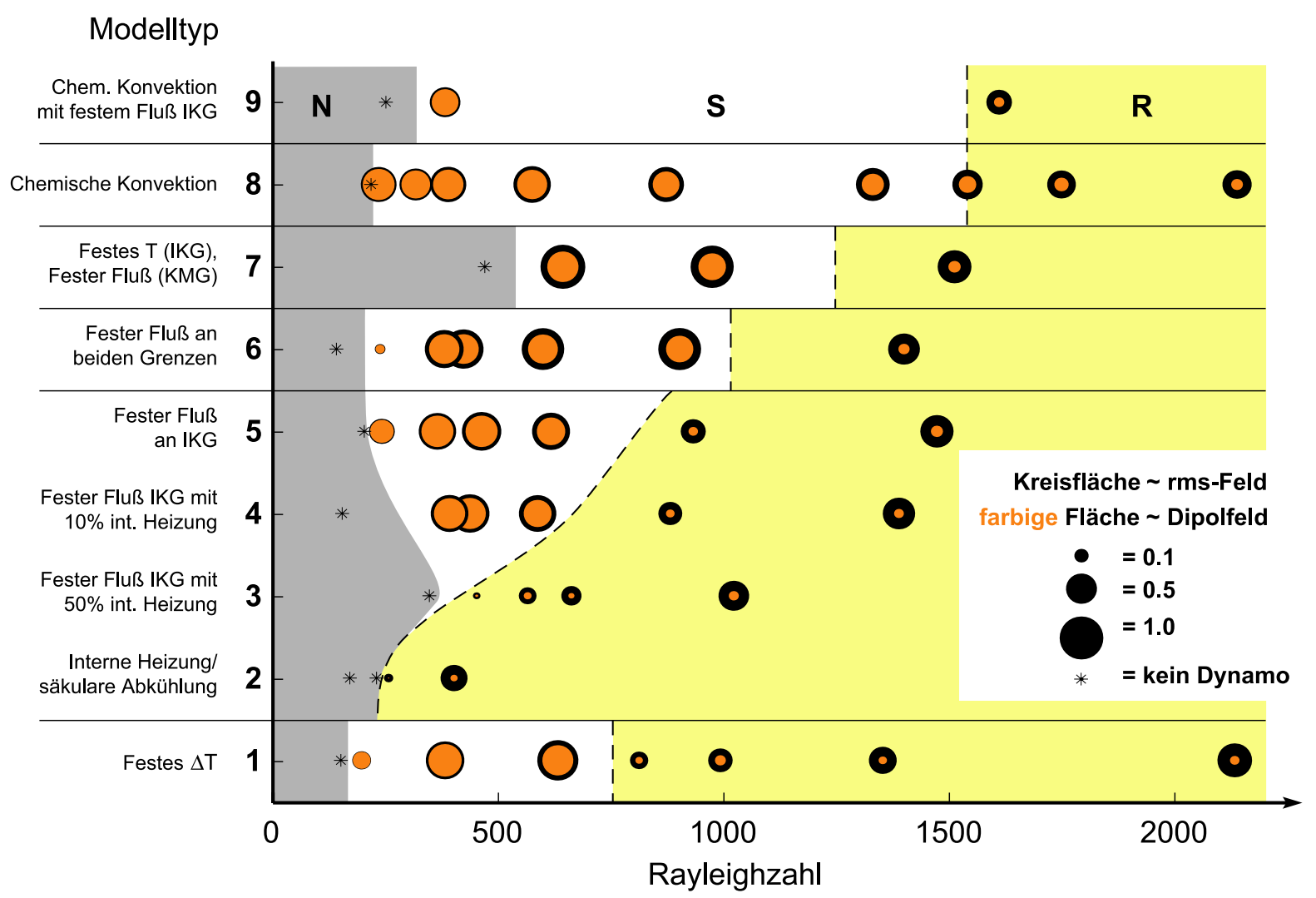

Abb. 3.7: $E=3 \times 10^{-4}, q=3$. Magnetfelder an der KMG für verschiedene Antriebsarten als Funktion der effektiven Rayleighzahl $\mathrm{Ra}^{\prime}$. Kreisflächen zeigen die zeitgemittelten Magnetfelder wie in Bild 3.5

magnetischen Bereich $\mathbf{N}$ haben Dipolanteile, die höchstens 2\% zur gesamten magnetischen Energie beitragen. Im Fall mit 100\% interner Heizung, $\mathrm{E}=10^{-4}$ und $\mathrm{q}=2$ hat die Lösung für das Magnetfeld eine quadrupolare Symmetrie $\left(B_{r}\right.$ symmetrisch zum Äquator) bei der niedrigsten Ra, für die es selbsterhaltende Dynamowirkung gibt. Auch Grote et al. (1999) finden in ihren intern geheizten Modellen bevorzugt quadrupolare Magnetfeldgeometrien, wenn die Prandtlzahl und die Robertszahl in der Größenordnung von 1 liegen.

Die Regimegrenze ist für die Antriebsarten 4-8 nicht so scharf wie für die Modelle mit festem $\Delta T$ (Typ 1). Bei steigender Ra gibt es vielmehr einen allmählichen Übergang von dipol-dominierten Dynamos hin zu solchen mit geringem Dipolbeitrag.

In Bild 3.8 werden gemittelte Energien für das Modell chemische Konvektion bei E = $3 \times 10^{-4}$ und $\mathrm{q}=3$ (Typ 8 aus Bild 3.7) gezeigt. Im Gegensatz zum Fall mit festem Temperaturkontrast (Abb. 3.6) schwankt die totale magnetische Energie nur wenig, sie bleibt sogar beim Überschreiten der Regimegrenze $\operatorname{Ra}_{\mathrm{R}}$ auf ähnlichem Niveau. Der Dipolbeitrag $(\ell=1)$ sinkt allerdings kontinuierlich mit steigender Ra. Für Ra bis 15-fach überkritisch ist die magnetische Energie höher als die kinetische, und die Dynamos haben stabile Polarität, wobei der Dipol 50-75\% zum rms-Feld an der KMG beiträgt. Der Dynamo bei Ra $=24 \times \mathrm{Ra}_{\text {krit }}$ ist generell stabil, doch gelegentlich fällt das Dipolmoment stark ab und eine Exkursion der Dipolachse tritt auf. Die Dynamos mit Ra $>30 \times \mathrm{Ra}_{\text {krit }}$ befinden sich im umkehrenden (R) Regime. Obwohl bei ihnen die magnetische Energie des Dipolanteils weniger als 2\% 


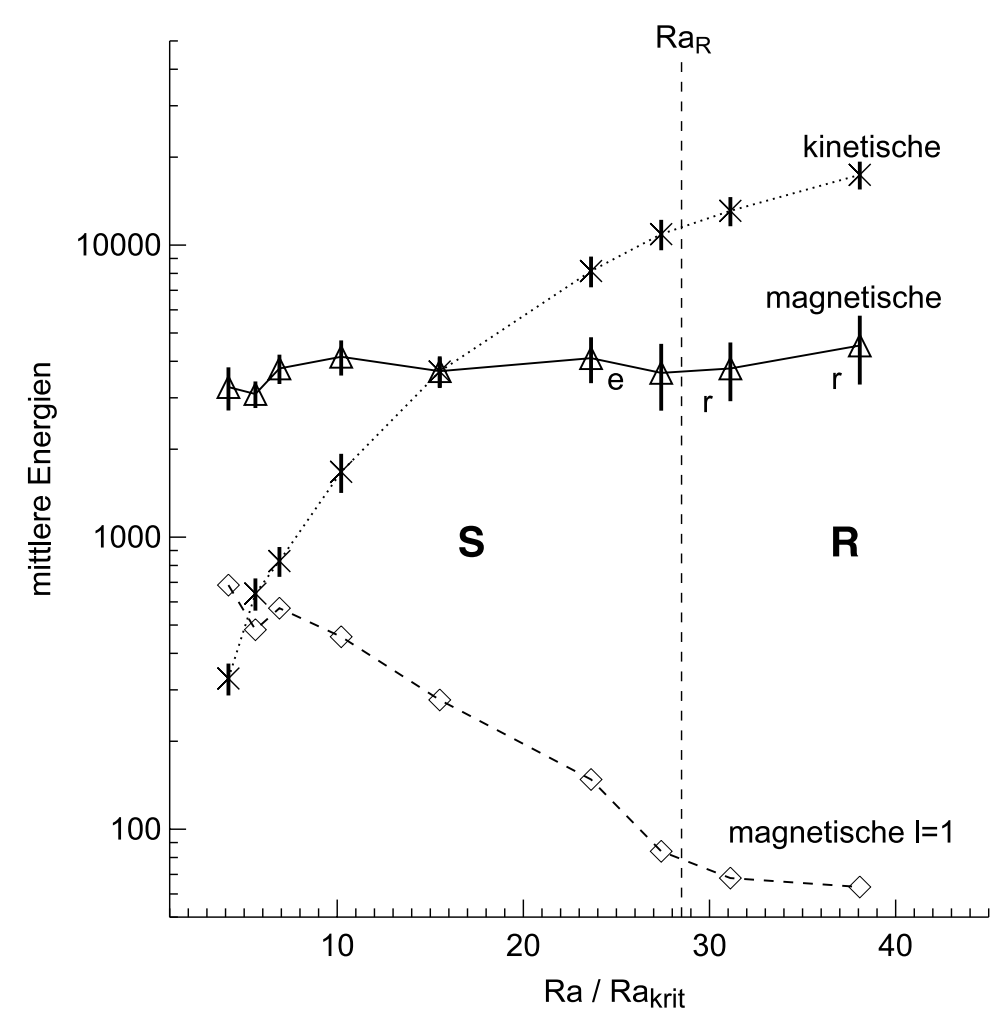

Abb. 3.8: Energiedichten als Funktion der Rayleighzahl für das Modelltyp 8 (chemische Konvektion) bei $\mathrm{E}=3 \times 10^{-4}, \mathrm{q}=3$. e: magnetischer Dipol zeigt Exkursionen, r: Dipol wechselt die Polarität. Die vertikalen Balken zeigen die Standardabweichung der zeitgemittelten Werte.

der gesamten magnetischen Energie in der Kugelschale ausmacht, ist das Feld an der KMG während langer Zeitspannen dipol-dominiert. Bei 31-fach überkritischer Ra trägt der Dipol durchschnittlich 25\% zum rms Feld bei, bei 38-fach überkritischer Ra noch $15 \%$.

Der Dynamo bei $\mathrm{Ra}=27 \times \mathrm{Ra}_{\text {krit }}$ zeigte während der 3.7 simulierten Diffusionszeiten weder eine Exkursion noch eine Umkehrung. Er hat aber im Vergleich zu allen anderen Modellen eine wesentlich höhere durchschnittliche Neigung der Dipolachse, nämlich über $10^{\circ}$. Wahrscheinlich weist dieser Dynamo bei längerer Simulationszeit auch Exkursionen oder Umkehrungen auf. Im Fall 24-fach überkritischer Ra konnten drei Exkursionen während der Laufzeit von 13.3 magnetischen Diffusionszeiten beobachtet werden. 

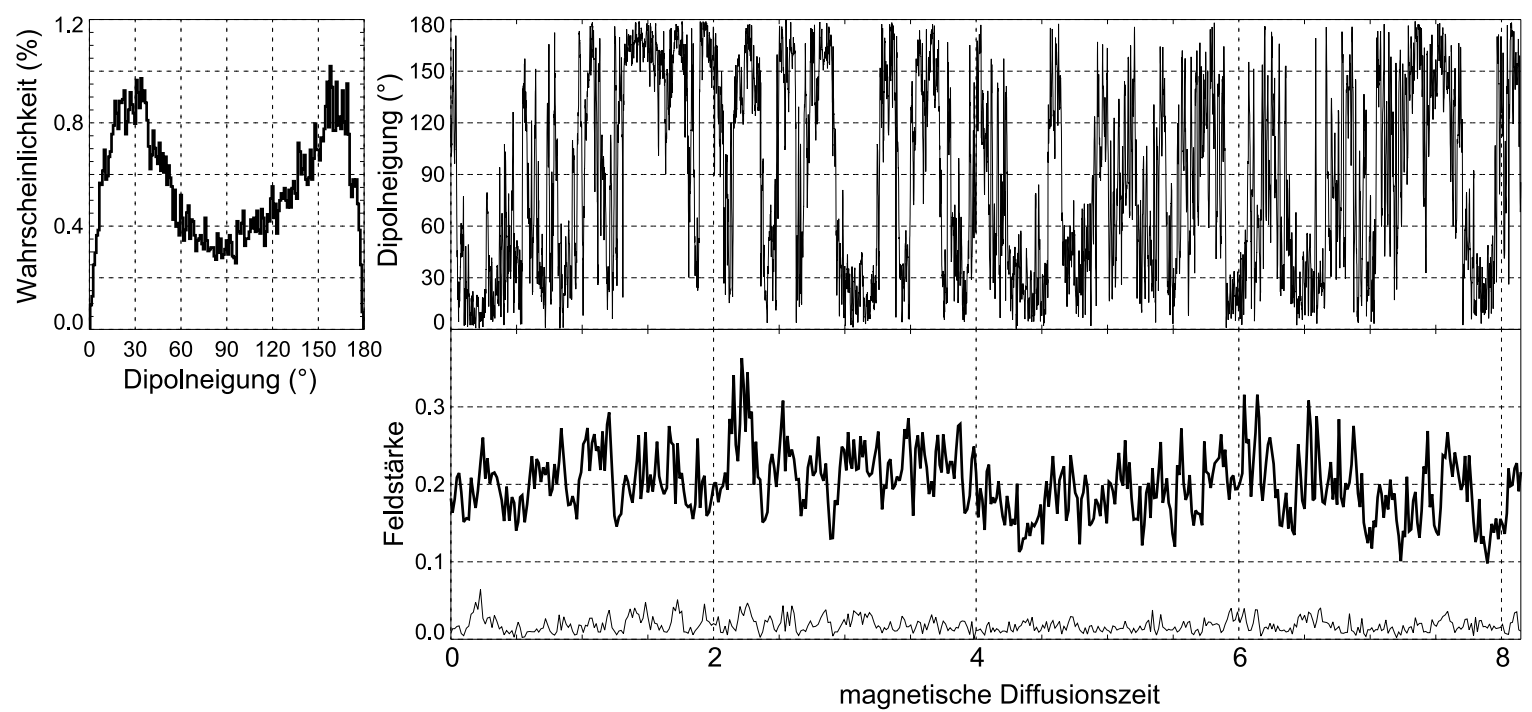

Abb. 3.9: Zeitreihe für festes $\Delta T$ (Typ 1) bei $E=3 \times 10^{-4}, \mathrm{q}=2, \mathrm{Ra}=22 \times \mathrm{Ra}_{\text {krit }}$. Oben: Neigung der Dipolachse an der KMG. Das Histogramm zur Linken zeigt die Wahrscheinlichkeit der Dipolneigung, in $1^{\circ}$ Breitenabschnitte zu fallen. Unten: Die dicke (dünne) Linie zeigt die gesamte (Dipol) rms-Feldstärke an der KMG.

\subsection{Polumkehrungen}

Für Dynamos, die sich weit hinter der Regimegrenze befinden $\left(\mathrm{Ra} \gg \mathrm{Ra}_{\mathrm{R}}\right)$ oder für die größtenteils $(\geq 50 \%)$ intern geheizten Modelle sind die Polaritätsänderungen des magnetischen Dipols nicht von großem Interesse, weil der Dipol nur einen geringen Beitrag zum Gesamtfeld leistet. Obwohl die Dipolneigung hier alle möglichen Winkel annimmt, zeigt der Dipol dennoch die Tendenz, sich entlang der Rotationsachse auszurichten. Bild 3.9 zeigt einen solchen Fall, bei dem der Dipol durchschnittlich nur 9\% zur gesamten rms-Feldstärke an der KMG beiträgt. Trotz extremer Fluktuationen der Dipolachse bleibt sie bevorzugt innerhalb $30^{\circ}$ der Rotationspole.

Die Dynamos, welche sich in der Nähe der Regimegrenze befinden $\left(\mathrm{Ra} \approx \mathrm{Ra}_{\mathrm{R}}\right)$, erzeugen für gewisse Zeitspannen eine signifikante Dipolkomponente. Das Zusammenbrechen des Dipols führt dann aber immer wieder zu Phasen mit sehr variabler Dipolrichtung. Die Phasen mit starkem Dipolbeitrag und stabiler Dipolrichtung wechseln sich in chaotischer Weise mit den Phasen ab, in denen der Dipol schwach ist und stark fluktuiert.

Bild 3.10zeigt das Zeitverhalten des Magnetfeldes an der KMG für die chemisch angetriebenen Dynamos aus Bild 3.8 bei drei verschiedenen Rayleighzahlen. Bei der niedrigsten Ra (16-fach überkritisch) ist die Dipolachse stabil und ihre Richtung stark an der Rotationsachse orientiert. Der Häufigste Neigungswinkel ist $3^{\circ}$, wobei Neigungswinkel von $14^{\circ}$ während der gesamten Simulationszeit nie überschritten werden. Die Dipolkomponente dominiert das KMG-Magnetfeld, wobei seine Intensität nur wenig schwankt.

Bei der mittleren Rayleighzahl (24-fach überkritisch) ist die Dipol-Dispersionskurve ein wenig breiter mit ihrem Maximum bei $5^{\circ}$. Die meiste Zeit ist der Dipol stabil, allerdings gibt es im Laufe der Simulation drei Ereignisse, während derer das Dipolmoment um ungefähr eine Größenordnung abfällt und die Dipolachse kurzzeitig den Äquator überquert. 

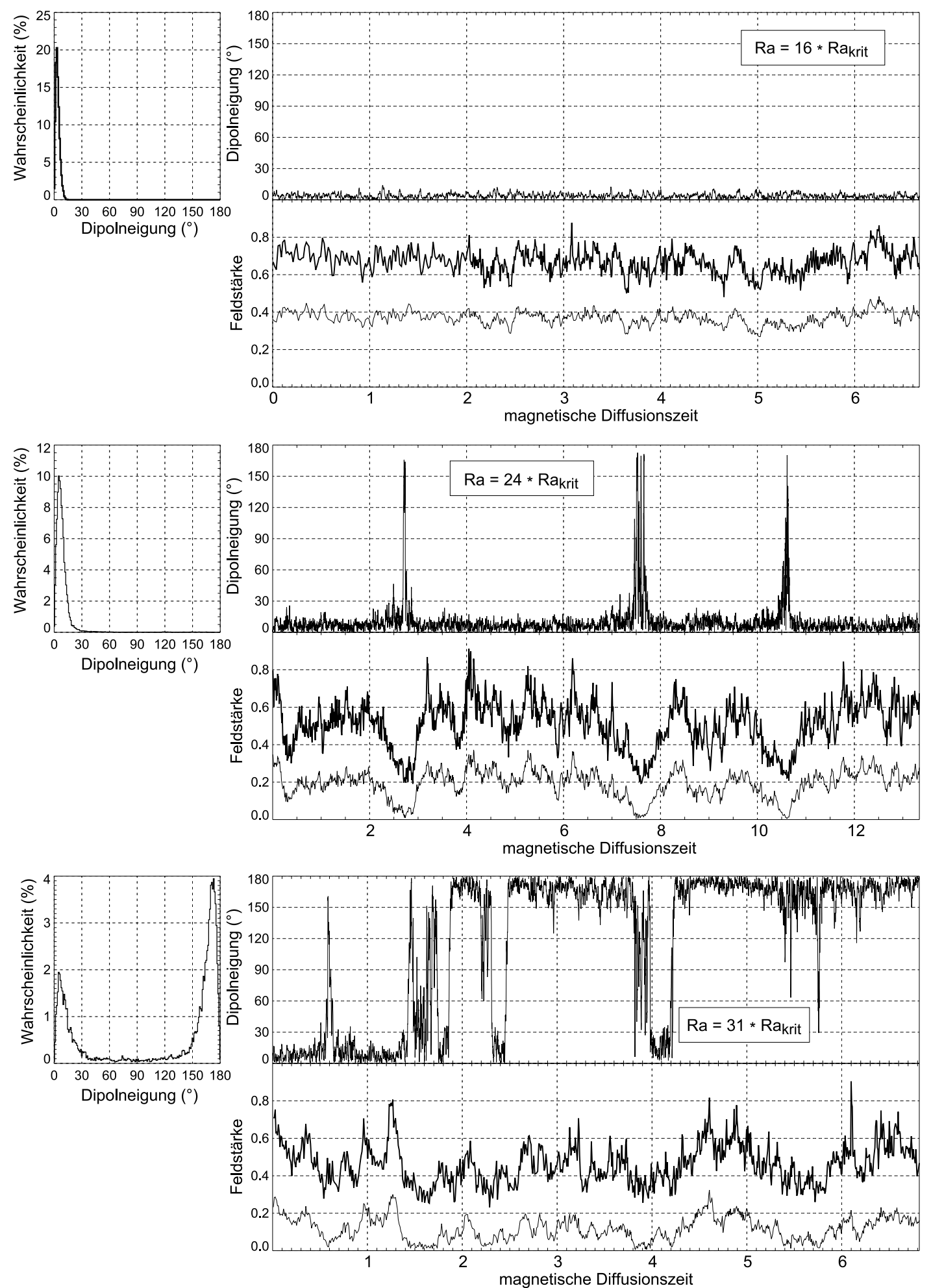

Abb. 3.10: Zeitreihen des KMG-Magnetfeldes für Modelltyp 8 (chemische Konvektion) bei $\mathrm{E}=3 \times 10^{-4}, \mathrm{q}=3$. Oben: Bei Ra $=16 \times$ Ra krit befindet sich der Dynamo im $\mathbf{S}$ Regime stabiler, dipol-dominierter Dynamos; Mitte: Exkursionen bei Ra $=24 \times$ $\mathrm{Ra}_{\text {krit }}$; Unten: Bei Ra $=31 \times \mathrm{Ra}_{\text {krit }}$ ist der Dynamo im umkehrenden $\mathbf{R}$ Regime. Jedes Einzelbild entspricht in der Darstellung der von Bild 3.9 Die Zeitreihe im mittleren Bild ist doppelt so lang. 
Die Zeitspannen, in denen Neigungswinkel größer als $45^{\circ}$ auftreten, dauern je nach Ereignis zwischen 0.05 und 0.25 magnetische Diffusionszeiten an, wobei die Zeitspannen geringer Dipolintensität jeweils etwas länger sind (0.2 bis 0.4 Diffusionszeiten). Da der Dipol sich in jedem der drei Fälle nach dem Ereignis in der gleichen Polarität wie vorher aufbaut, sollen diese Ereignisse als Exkursionen bezeichnet werden. In der Zeit zwischen zwei Exkursionen hat das Dipolfeld eine durchschnittliche Amplitude von 40-50\% des Gesamtfeldes. Die Änderungen der Gesamtfeldstärke an der KMG können größtenteils auf die Fluktuationen der Dipolintensität zurückgeführt werden, wobei die höheren Multipolbeiträge während der Exkursionen auch abnehmen, allerdings weniger stark. Die Schwankungen der rms-Feldstärke um den Mittelwert sind hier größer als im Fall niedriger Ra.

Im umpolenden Fall mit der höchsten Rayleighzahl (31-fach überkritisch) häufen sich die Neigungswinkel bei $7^{\circ}$ und $173^{\circ}$. Neben vollständigen Polumkehrungen finden sich auch wie im Fall 24-fach überkritischer Ra einige Exkursionen, z. B. bei $t \approx 0.6$ und $t \approx 5$.5. Sowohl das mittlere KMG Feld als auch der Relativbeitrag des Dipols sind kleiner als in den anderen beiden Beispielen. Zeiträume mit einem schwachen Dipol, welche typischerweise 0.3 magnetische Diffusionszeiten lang andauern, sind korreliert mit unsystematischen Fluktuationen im Neigungswinkel des Dipols. Zeiten, in denen die Dipolachse nur wenige Grad von der Rotationsachse abweicht, sind korreliert mit großen Beiträgen des Dipols zum Gesamtfeld. Nach den ungefähr 7 magnetischen Diffusionszeiten, welche durch die Simulation abgedeckt werden, sind die Magnetfeldpolaritäten noch nicht gleich verteilt. Die ungleich hohen Maxima in der Dispersionskurve des Neigungswinkels deuten darauf hin, daß es sehr niedrige Frequenzen im Zeitverhalten dieses Dynamos gibt.

Neben Zeitspannen mit chaotisch fluktuierender Dipolrichtung gibt es auch einige klar definierte und schnelle Umkehrungen zwischen dipolaren Zuständen gegensätzlicher Polarität, z. B. um $t=1.85, t=2.45$ oder $t=4.2$. Bild 3.11zeigt Momentaufnahmen des radialen magnetischen Feldes $B_{r}$, fortgesetzt nach oben, so wie es an der Erdoberfläche aussehen würde. Der Verlauf der Dipolneigung und der Magnetfeldstärke an der KMG kann für den relevanten Zeitraum in Bild 3.12 betrachtet werden. Anders als in Bild 3.10 ist nur die rms-Feldstärke der ersten elf harmonischen Moden aufgetragen. Dies ist besser für den Vergleich mit dem Magnetfeld des Erdkerns geeignet, dessen Energiegehalt in kleineren Skalen aufgrund der Abschirmung durch das Krustenfeld unbekannt ist. Das Feld wechselt von normaler Polarität zum Zeitpunkt $t_{1}$ zu inverser Polarität bei $t_{3}$. Dazwischen liegt ein Zeitraum mit schwachem Dipolfeld und unterdurchschnittlich kleinem Gesamtfeld (die Zeit um $t_{2}$ ). Während dieser Übergangsperiode ist das Feld an der Erdoberfläche dominiert von Beiträgen höherer Multipole.

Das KMG-Feld der Simulation (Fig. 3.13) ist recht kleinskalig, wenn man es mit aktuellen Modellen des Kernfeldes der Erde vergleicht (z. B. Jackson et al., 2000). In einem Film, welcher das KMG-Feld der Simulation während der Umpolung zeigt, sieht man zu jeder Zeit viele kleine Flußbündel, die an verschiedenen Stellen auftauchen, sich bewegen und schließlich wieder in ihrer Intensität abnehmen und verschwinden. Sie können beliebige Polarität in der jeweiligen Hemisphäre haben, doch es gibt normalerweise entweder mehr normale oder mehr inverse Flußbündel, was dann die Polarität des Dynamos ausmacht. Betrachtet man z. B. den Schnappschuß aus Bild 3.13 kurz vor der Umkehrung, so sieht man, daß positives $B_{r}$ in der Nordhemisphäre dominiert, wobei es aber auch einige ausgebildet starke Flußbündel mit negativem $B_{r}$ gibt. Vor der Umpolung verschwindet diese Dominanz einer Polarität 
$t_{1}$
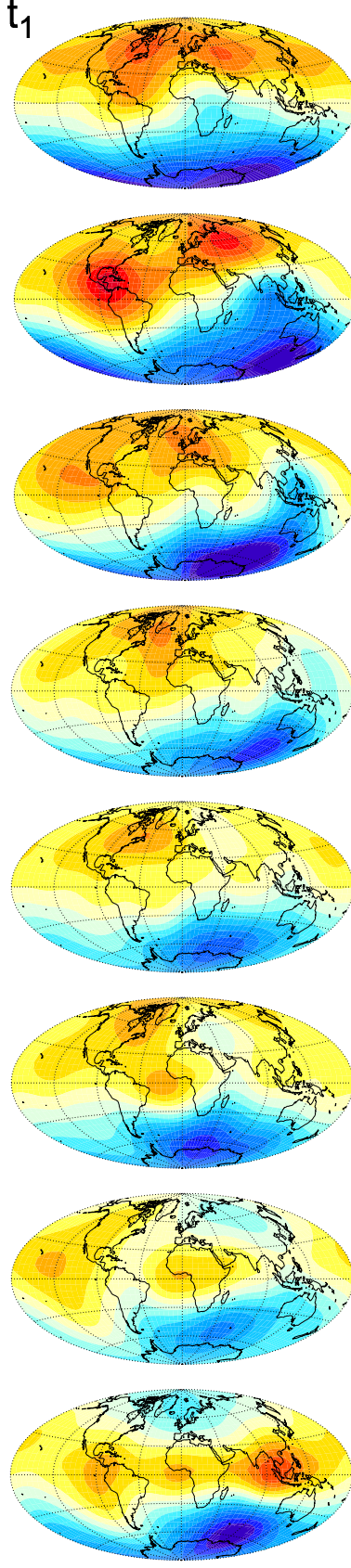
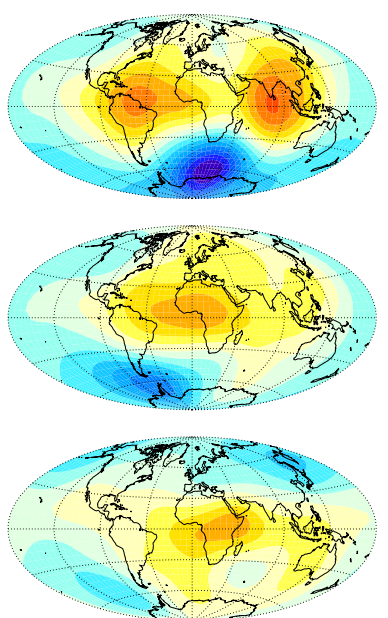

$t_{2}$
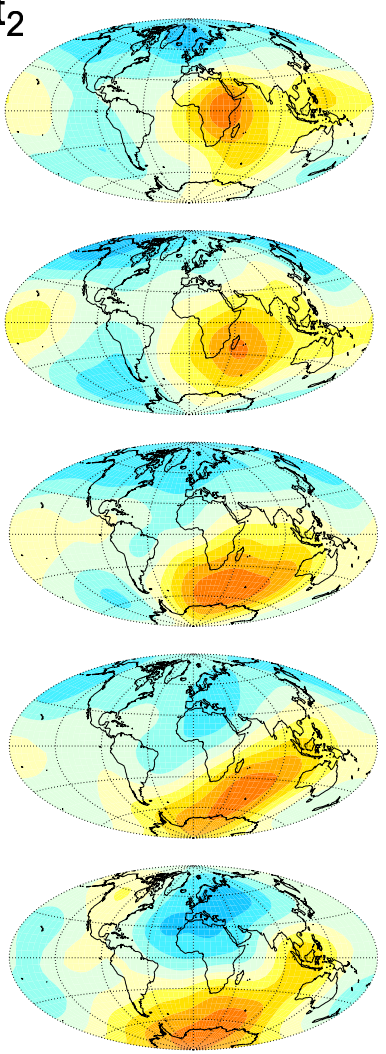
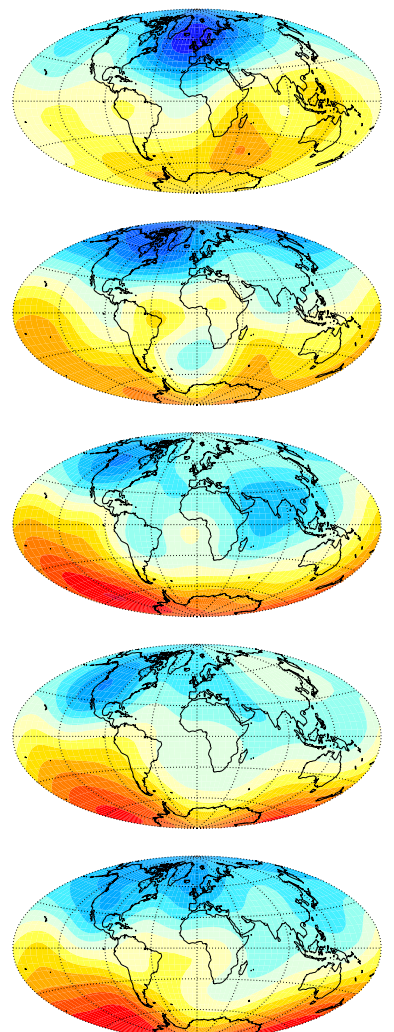

$t_{3}$

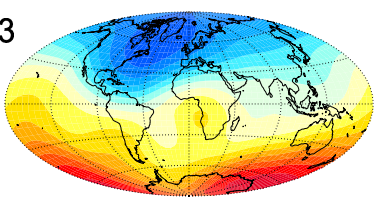

Abb. 3.11: Radiales Magnetfeld $B_{r}$ des Dynamos aus Bild 3.10 unten $\left(\mathrm{Ra}=31 \times \mathrm{Ra}_{\mathrm{krit}}\right)$, so wie es an der Erdoberfläche erscheinen würde. Blau negatives, rot positives $B_{r}$, ein Konturschritt entspricht einem $\Delta B_{r}$ von 0.004. Die Schnappschüsse sind in Abständen von etwa 0.003 magnetischen Diffusionszeiten genommen (das entspricht bei der gewählten Skalierung etwa 500 Jahren). Die Kontinente dienen nur dazu, die Skalen der Magnetfeldstrukturen einzuordnen. 


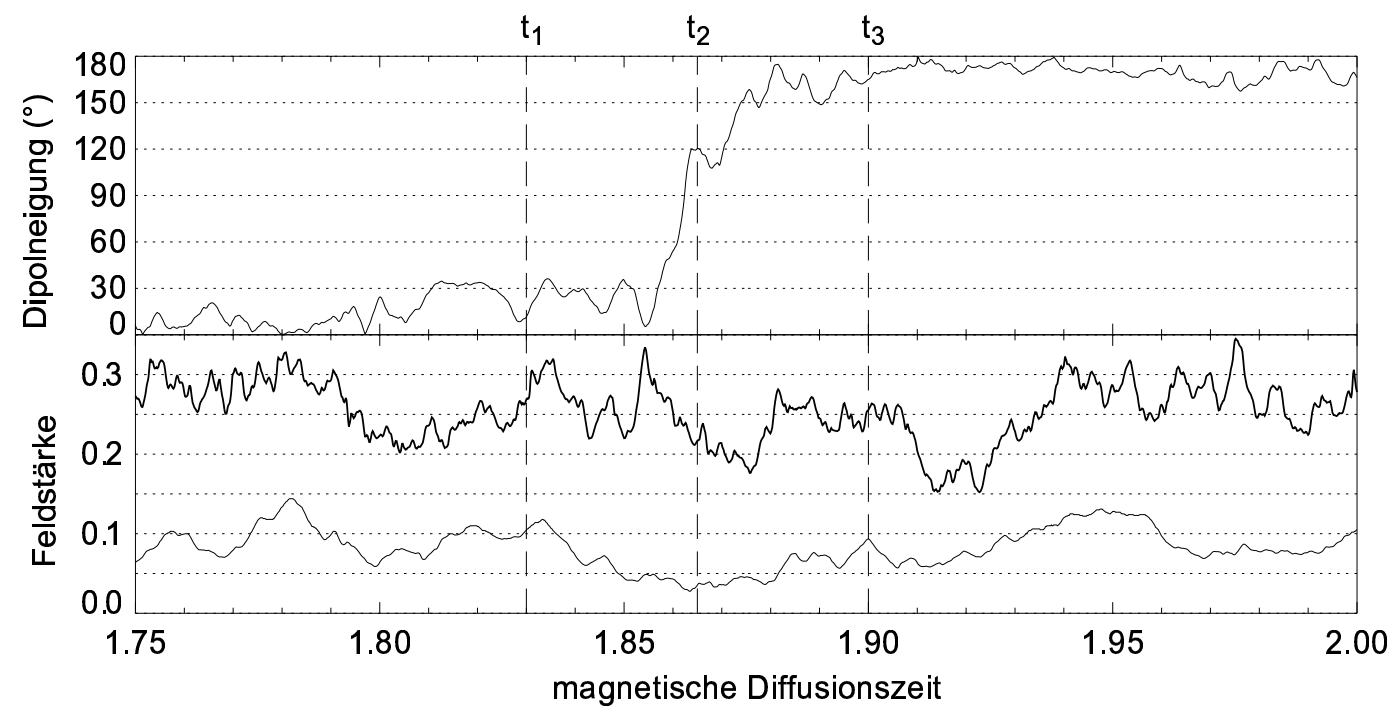

Abb. 3.12: Ausschnitt aus Bild 3.10, unten. Oberer Teil: Zeitreihe der Dipolneigung. Unterer Teil: Dicke Linie zeigt die rms-Feldstärke in den harmonischen Moden von $\ell=$ $1 \ldots 11$, dünne Linie die rms-Feldstärke des Dipols $(\ell=1)$. Die Zeitspanne aus Bild 3.11 ist mit den gestrichelten vertikalen Linien markiert $\left(t_{1}-t_{3}\right)$.

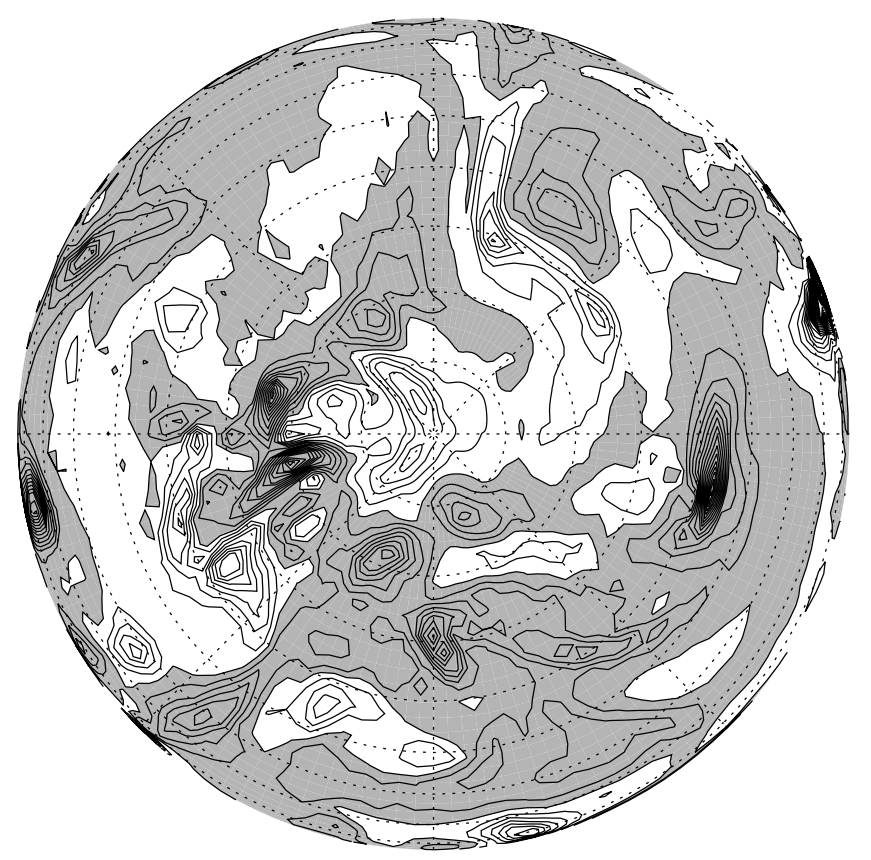

Abb. 3.13: $B_{r}$ an der KMG für den Dynamos aus Bild 3.12 Blick auf den Nordpol zur Zeit $t=1.835$. Positives (negatives) $B_{r}$ innerhalb schattierter (weißer) Bereiche, ein Konturschritt entspricht $\Delta B_{r}=0.16$. 
immer mehr, so daß in der Mitte der Umkehrung Flußflecken jeder Polarität gleichberechtigt existieren. Irgendwann tauchen schließlich ein oder zwei starke Flußbündel der inversen Polarität auf, welche dann das Feld in dieser Hemisphäre dominieren. Das Gesamtverhalten macht einen sehr lebendigen Eindruck, da auf kleinen Skalen ständig sehr viel passiert. Generell bilden sich Flußbündel jedoch bevorzugt in äquatornahen Regionen und wandern dann in eine nordwestliche Richtung.

$\mathrm{Ab}$ und zu taucht ein Paar Flußbündel entgegengesetzter Polarität in polaren Regionen auf, welches darauf schnell wieder zerfällt. Ein solches Ereignis ist kurz nach Beginn der Polumkehr bei $t \approx 1.866$ zu beobachten und es ist korreliert mit einer plötzlichen Zunahme des polaren Aufstroms (auch Plume genannt). Sarson \& Jones (1999) deuteten diese sogenannten „,buoyancy surges“ als Auslöser ihrer Polumkehrungen. Hier scheint erhöhter Plumefluß aber keine wesentliche Rolle für die Umpolung zu spielen, welche deutlich vor dem Auftreten des starken Plumes beginnt. Erhöhter Fluß in den polaren Plumes kann in diesem Modell auch in Phasen mit stabiler Feldpolarität beobachtet werden. Das Paar entgegengesetzter Flußbündel kann als (ursprünglich) toroidales Feld gedeutet werden, welches durch den starken Aufstrom mitgeführt und zum Austreten aus dem Kern gebracht wird. Läßt der Aufstrom nach, verschwinden diese beiden Flußbündel schnell wieder.

\subsection{Vergleich mit der Erde}

Um das Modell auf die Erde zu skalieren, wird für den Kern eine Dichte von $10^{4} \mathrm{~kg} / \mathrm{m}^{3}$ und eine Leitfähigkeit von $6 \times 10^{5} \mathrm{~S} / \mathrm{m}$ angenommen. Ein dimensionsloser Wert des Dipolfeldes an der KMG von 0.1 entspricht dann einem Dipolmoment von etwa $3.3 \times 10^{22} \mathrm{Am}^{2}$. Die durchschnittlichen Dipolmomente der chemisch angetriebenen Modelle aus Bild 3.10 sind 12.0, 6.4 und $3.8 \times 10^{22} \mathrm{Am}^{2}$ entsprechend 16-, 24- und 31-fach überkritischer Rayleighzahl. Das durchschnittliche Dipolmoment des Modells mit der höchsten Ra ist also etwas höher als das der Erde heute $\left(8 \times 10^{22} \mathrm{Am}^{2}\right)$, aber es kann diesen Wert in Zeiten mit starkem Dipolfeld auch übertreffen. Aus paläomagnetischen Messungen ist bekannt, daß das über lange Zeiten gemittelte Dipolmoment der Erde im Bereich von $(4-6) \times 10^{22} \mathrm{Am}^{2}$ liegt (Dormy et al., 2000), s. dazu Abb. 3.14.

Das Dipolmoment des umkehrenden Dynamos liegt im Vergleich dazu etwas zu niedrig, wogegen das des Dynamos mit Exkursionen etwas zu hoch ist. Chaotische Variationen der Dipolstärke finden sich auf verschiedenen Zeitskalen für jedes der Modelle aus Bild 3.10 , allerdings ist die Amplitude der Variationen am ausgeprägtesten für die Modelle höherer Ra.

In Bild 3.15] werden die Spektren des Magnetfeldes an der KMG für verschiedene Zeitspannen des umkehrenden Modells mit dem Feld der Erde 1980 verglichen. In Zeiten mit stabilem axialen Dipol stimmt das Spektrum des Modells recht gut mit dem der heutigen Erde überein. Der durchschnittliche Dipolbeitrag $(\ell=1)$ ist im Modell allerdings etwas geringer, wobei der Energiegehalt in den Moden $\ell \geq 8$ etwas höher ist. Während Exkursionen und Umkehrungen ist der Dipolbeitrag kleiner als der der Moden $\ell=2 \ldots 11$.

Um die Umkehrfrequenz des Modells mit der geomagnetischen Polaritäts-Zeitskala (Abb. $1.1 \mathrm{zu}$ vergleichen, muß definiert werden, was eine vollständige Polumkehrung ausmacht. Perioden stark fluktuierender Dipolrichtung sollen ausgeschlossen werden, weil sie nicht aussagekräftig sind, solange die Dipolintensität niedrig ist. Es wird gefordert, daß nicht nur 


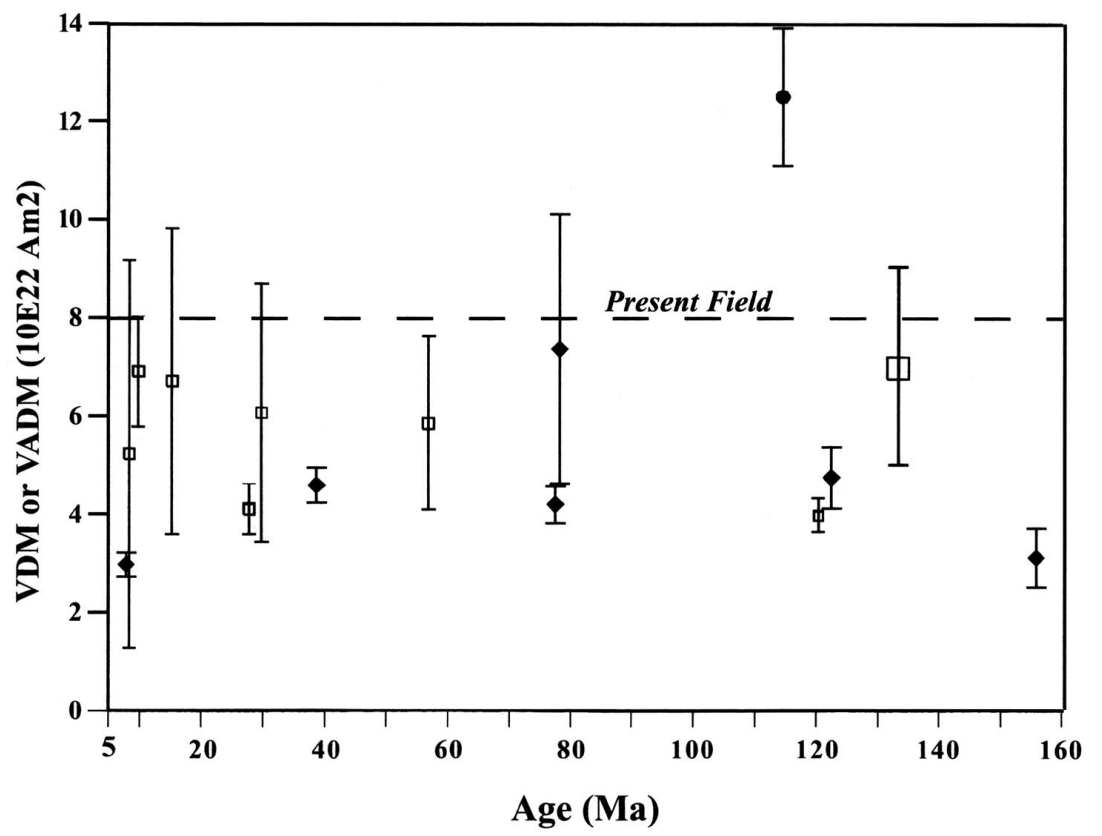

Abb. 3.14: Virtuelles Dipolmoment (Quadrate, Kreise) und virtuelles axiales Dipolmoment (Rauten) für Zeiten von 5 bis 160 Mio. Jahre vor der Gegenwart. Nach Goguitchaichvili et al. (2002). Das virtuelle Dipolmoment (VDM) erhält man, indem man aus den magnetischen Elementen $(F, I, D)$ neben der Neigung des virtuellen geozentrischen Dipols auch dessen Dipolmoment berechnet, welches an der Site die beobachtete Totalintensität $F$ hervorrufen würde.

die Dipolachse den Äquator überquert, sondern auch, daß die Dipolfeldstärke in der neuen Polarität zu einem gewissen Zeitpunkt 0.1 überschreitet. Im Modell mit 31-fach überkritischer Rayleighzahl (Bild 3.10 unten) ergibt das mit dieser Definition 5 Umkehrungen in 7 magnetischen Diffusionszeiten, was etwas höher ist, als die durchschnittliche paläomagnetische Umkehrfrequenz. Natürlich ist die Laufzeit der Simulation noch nicht lange genug für eine verläßliche Statistik der Umkehrfrequenz.

Definiert man als Dauer einer Umpolung die Zeitspanne, in der die Dipolachse um mehr als $30^{\circ}$ von der Rotationsachse abweicht, erhält man eine Dauer von 0.01 bis 0.04 für die Umkehrungen aus Bild 3.10 (siehe auch Bild 3.12). Mit der gewählten Skalierung übersetzt sich das in 1500 bis 6500 Jahre, was mit Abschätzungen über die Dauer von geomagnetischen Umpolungen übereinstimmt (Merrill \& McFadden, 1999). Eine weitere Übereinstimmung ist die Tatsache, daß Umkehrungen mit Phasen schwacher Dipolintensität korreliert sind, welche ausgedehnter sind, als die Phasen hoher $\left(>30^{\circ}\right)$ Dipolneigung. Auch ist die Feldstruktur nicht-dipolar während der Umkehrungen. Exkursionen sind den vollständigen Umkehrungen im Modell sehr ähnlich, nur daß sich der Dipol in der gleichen Polarität wieder aufbaut, die er vor seinem Intensitätsminimum hatte.

Obwohl mehrere Aspekte des umkehrenden Modells gut mit dem Erdmagnetfeld übereinstimmen, gibt es in der Simulation auch ausgedehnte Phasen niedriger Dipolintensität bei 


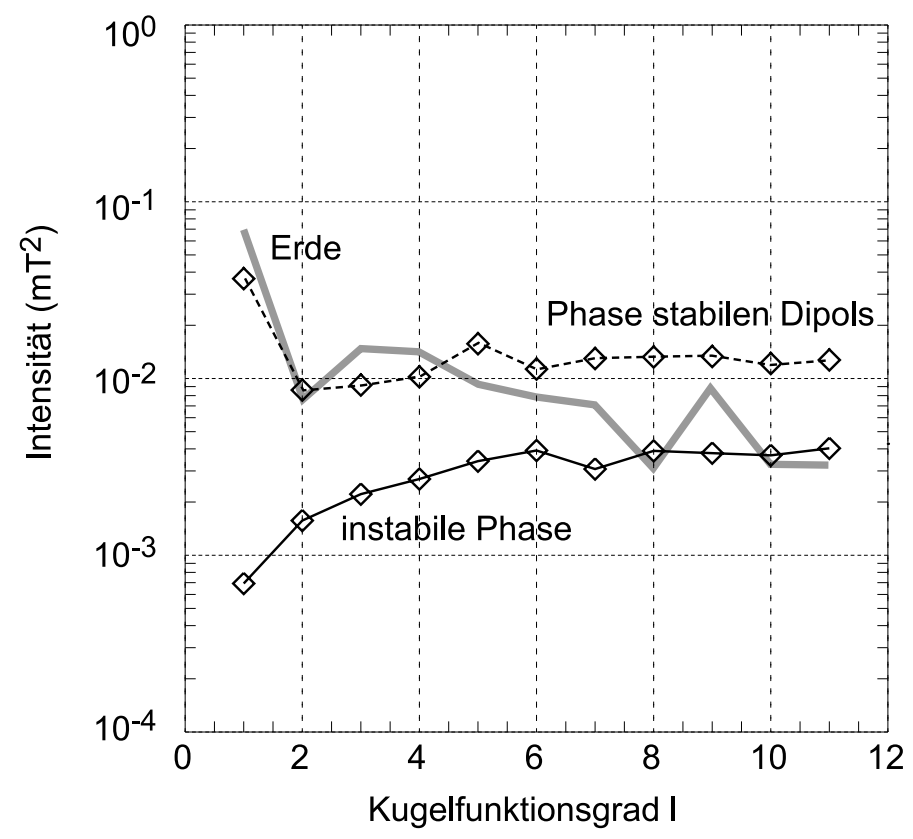

Abb. 3.15: Spektrum des KMG-Magnetfeldes als Funktion des harmonischen Grades $\ell$ für die Erde 1980 (dicke graue Linie, DGRF-Daten aus Jacobs (1994)) und für das umkehrende Modell aus Bild 3.10, unten (schwarze Linie mit Rauten). Gepunktet: Zeitmittel einer Phase mit stabiler Dipolachse $(t=4.5 \ldots 5.2)$. Durchgezogen: Zeitmittel einer Phase mit stark fluktuierender Dipolrichtung $(t=1.5 \ldots 1.7)$.

stark schwankender Dipolrichtung (z. B. zwischen $t=1.5$ und $t=1.7$ ), welche sich nicht in paläomagnetischen Aufzeichnungen finden. 


\section{Der Einfluß des Mantels auf den umpolenden Geodynamo}

Es soll jetzt der Einfluß verschiedener an der KMG vorgegebener Wärmeflußmuster auf umkehrende Dynamos untersucht werden. Die in diesem Kapitel beschriebenen Modelle unterscheiden sich deswegen von den vorangegangenen dadurch, daß an der äußeren Grenze eine Flußrandbedingung vorgegeben wird. Gleichzeitig wird eine Modifikation der Antriebsart eingeführt, die es erlaubt, thermische und chemische Konvektion kombiniert zu behandeln. Wir betrachten Modelle, die chemisch angetrieben werden, und die zusätzlich an ihrer äußeren Grenze einem vorgegebenen (thermischen) Fluß unterliegen.

Für diese Modelle wird neben der tatsächlichen Dipolneigung die Lage der virtuellen geomagnetischen Pole betrachtet, welche im Paläomagnetismus aus den an der Erdoberfläche vorliegenden Daten bestimmt werden. Aus einer Messung der Deklination und der Inklination des Magnetfeldvektors an einem bestimmten Ort (der Site), kann der VGP für diesen Ort bestimmt werden. Der VGP ist definiert als der Südpol eines gedachten geozentrischen Dipols, der am Ort der Messung die gleiche Inklination und Deklination ergeben würde,

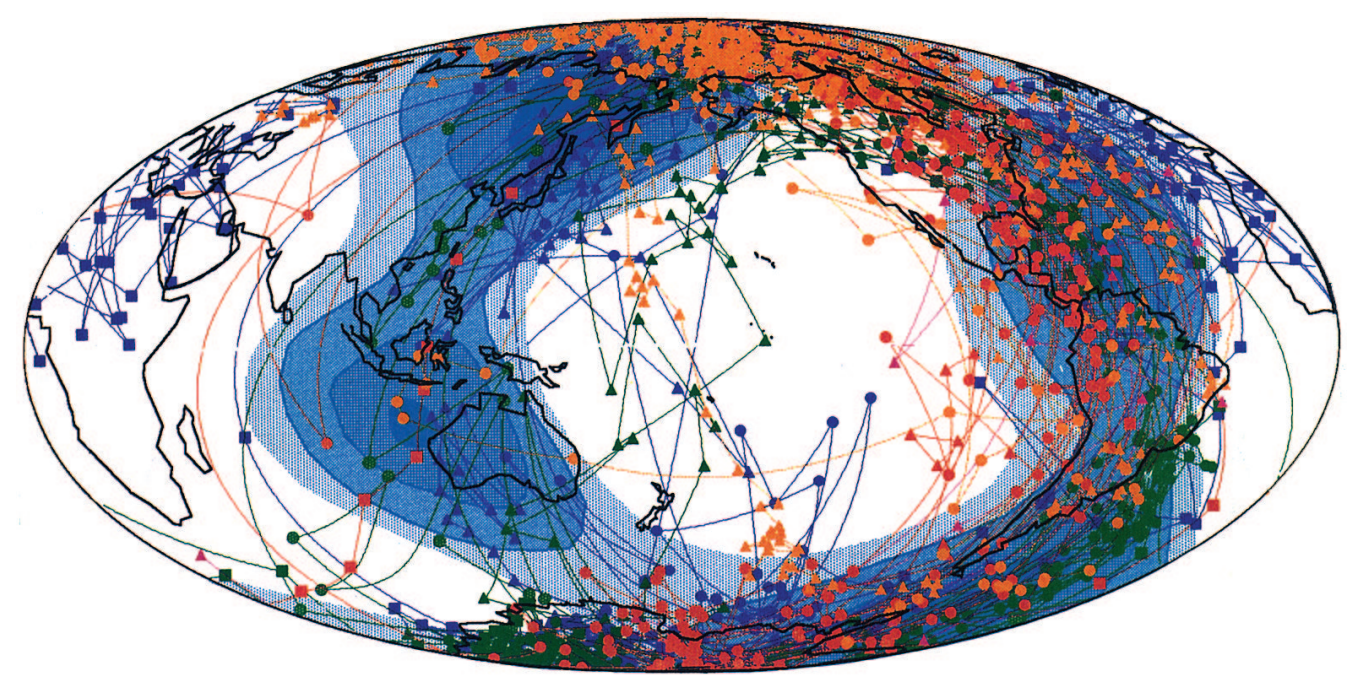

Abb. 4.1: Symbole: VGP Trajektorien für das sog. Blake-Ereignis (115 000-120 000 Jahre vor Heute), die jüngere Olduvai-Umkehr (vor $\approx 1.8$ Mio. Jahren) und zwei Umkehrungen vor 6.5 und 11 Mio. Jahren. Farblich unterlegt: Seismische Kompressionswellengeschwindigkeit nach Dziewonski \& Woodhouse (1987) im unteren Mantel in einer Tiefe von $2300 \mathrm{~km}$. (Lai et al., 1991). 
wie die tatsächlich beobachtete $I$ und $D$. Für die Vergangenheit kann der Magnetfeldvektor z. B. aus Sedimenten oder Laven rekonstruiert werden, welche zu ihrer Entstehungszeit die am Ort ihrer Entstehung herrschende Feldrichtung aufgezeichnet haben. Eine ausführliche Zusammenstellung der dazu nötigen paläomagnetischen Techniken gibt z. B. Tauxe (1998). Wäre das Erdmagnetfeld ein reines Dipolfeld, so würde man (bei gegebener Feldkonfiguration) für jede Site den gleichen VGP erhalten, welcher in diesem speziellen Fall identisch mit dem einen Durchstoßpunkt der Dipolachse durch die Erdoberfläche wäre. Hat das Feld zusätzlich nicht-dipolare Komponenten, so werden die VGP's Site-abhängig. Je stärker die Feldkonfiguration von einem Dipolfeld abweicht, desto stärker wird die Streuung der VGP's sein.

Mit den Simulationen soll herausgefunden werden, ob ein an der KMG vorgegebener inhomogener Wärmefluß zu einer Häufung der virtuellen Pole führt, und ob bei Umkehrungen des Feldes die VGP's bestimmte Trajektorien bevorzugen. Als Trajektorie soll hier der Pfad bezeichnet werden, den die virtuellen Pole auf der (Erd-)oberfläche beschreiben. Es werden insbesondere die transienten VGP's von Interesse sein, welche während Exkursionen oder Umkehrungen des Feldes auftreten. Ein VGP soll als transient bezeichnet werden, wenn er mindestens $30^{\circ}$ von jedem Rotationspol entfernt liegt.

Ein Beispiel, wie die Verteilung der VGP's für einige Umkehrungen der letzten 11 Mio. Jahre aussehen könnte, wurde von Laj et al. (1991) gegeben und ist in Abb. 4.1 gezeigt. (Das Blake-Ereignis heißt deswegen „Ereignis“, weil es nicht eindeutig als Exkursion oder Umkehrung klassifiziert werden kann.) Man erkennt sowohl die Häufung der VGP's entlang amerikanischer und ostasiatischer Längen als auch die hohe Korrelation mit der Geschwindigkeit seismischer Wellen im unteren Mantel. Die Farben Rot, Blau, Grün und Orange sind einzelnen Umkehrungen zugeordnet.

Die in Lai et al. (1991) beobachtete VGP-Häufung ist allerdings umstritten und wurde u. a. von Valet et al. (1992) als mögliches Artefakt kritisiert, welches durch die Datenbearbeitung entstanden sein könnte. Um zu verhindern, daß Trajektorien mit einer großen Anzahl an VGP's das Gesamtbild verzerren, berechnen Valet et al. eine mittlere Länge für jede VGP-Trajektorie. Für unterschiedliche Sites finden sie unterschiedliche Längen, entlang derer die VGP's fallen. Aus ihrer Untersuchung ergibt sich kein statistischer Nachweis dafür, daß VGP's verschiedener Umkehrungen die gleichen Längenintervalle bevorzugen.

Prévot \& Camps (1993) betrachten Exkursionen und Umkehrungen der letzten 16 Mio. Jahre, die in vulkanischem Gestein aufgezeichnet wurden. Die Untersuchung der etwa 400 transienten VGP's ergibt allerdings keinen Hinweis auf eine Häufung, sondern vielmehr eine gleichmäßige Verteilung der VGP's über die Längen. Love (1998) greift auf eine etwas gröBere Datenbasis zurück: etwa 1300 transiente VGP's aus vulkanischen Datensätzen, in denen Umkehrungen und Exkursionen der letzten 20 Mio. Jahre aufgezeichnet sind. Er findet, daß auch die transienten VGP's der vulkanischen Datensätze bevorzugt entlang amerikanischer und asiatischer Längen fallen und in etwa mit den Ergebnissen aus den Sediment-Daten übereinstimmen.

Abhängig davon, wie die virtuellen Pole während einer Umpolung verteilt sind, lassen sich Aussagen über die Geometrie des Erdmagnetfeldes in dieser Phase machen. Sehen verschiedene Sites für dieselbe Umkehr stark unterschiedliche Trajektorien, kann das Feld nicht dipolar gewesen sein. Clement (1991), Clement \& Kent (1991) und Hoffman (2000) finden z. B. für die Matuyama-Brunhes-Umkehr transiente VGP's sowohl entlang amerikanischer 

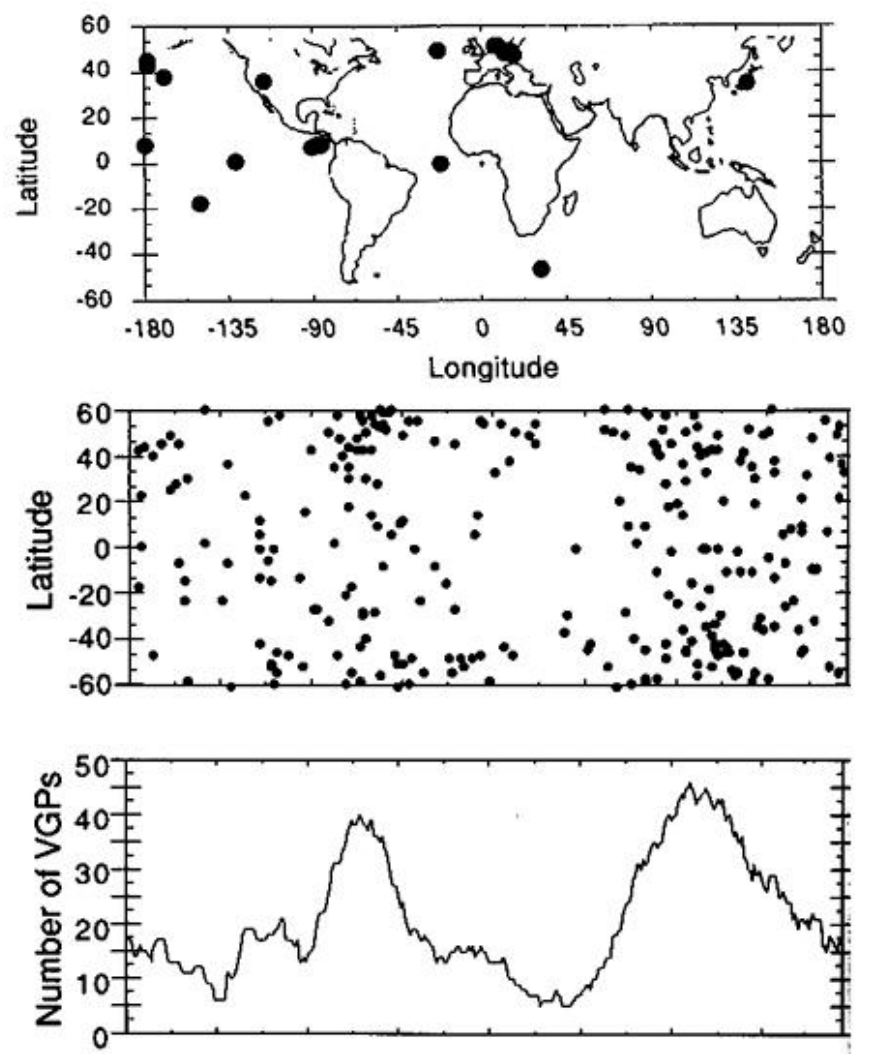

Abb. 4.2: Verteilung der transienten VGP's für die Matuyama-Brunhes Umkehr, nach Clement (1991). Oben: Lage der Sites, an denen Daten vorliegen. Mitte: Geographische Verteilung der für diese Daten berechneten VGP's. Unten: Anzahl der transienten VGP's in einem $30^{\circ}$ Längenfenster.

als auch entlang ostasiatischer Längen (Abb. 4.2 und 4.3). Clement weist darauf hin, daß die von den VGP's bevorzugten Längen jene Längen sind, bei denen sich im historischen Erdmagnetfeld starke Flußkonzentrationen befinden.

Neben den VGP's ist auch die Position der starken Flußbündel des Magnetfeldes möglicherweise von der Lage kalter Regionen im unteren Mantel abhängig: Olson \& Christensen (2002) betrachten das zeitgemittelte Magnetfeld einer Dynamo-Simulation mit einem aus der seismischen Tomographie abgeleiteten Wärmeflußmuster an der KMG. Sie finden Konzentrationen des magnetischen Flusses in der Nähe der Stellen, an denen das zeitgemittelte historische Erdmagnetfeld (Jackson et al., 2000) starke Flußbündel aufweist.

An einem kinematischen, umkehrenden Dynamo-Modell demonstrierten Gubbins \& Sarson (1994), daß solche Flußkonzentrationen zu Häufungen der VGP's führen können. Das Magnetfeld ihres Modells ist antisymmetrisch zur Äquatorebene; es zeichnet sich durch zwei Paare an Flußbündeln aus, ähnlich wie sie im heutigen Magnetfeld oder im zeitgemittelten Paläomagnetfeld der letzten 2.5 Millionen Jahre zu finden sind. Die VGP-Trajektorien, welche sie für die Umkehrung des Modellfeldes berechnen, sind stark Site-abhängig. Sie liegen jeweils sehr nahe an den Längen, in denen die starken Flußkonzentrationen zu finden sind; es ergeben sich also VGP-Häufungen entlang amerikanischer und asiatischer Längen.

Ein möglicher Kopplungsmechanismus zwischen Mantel und Kern sind Gebiete niedriger 


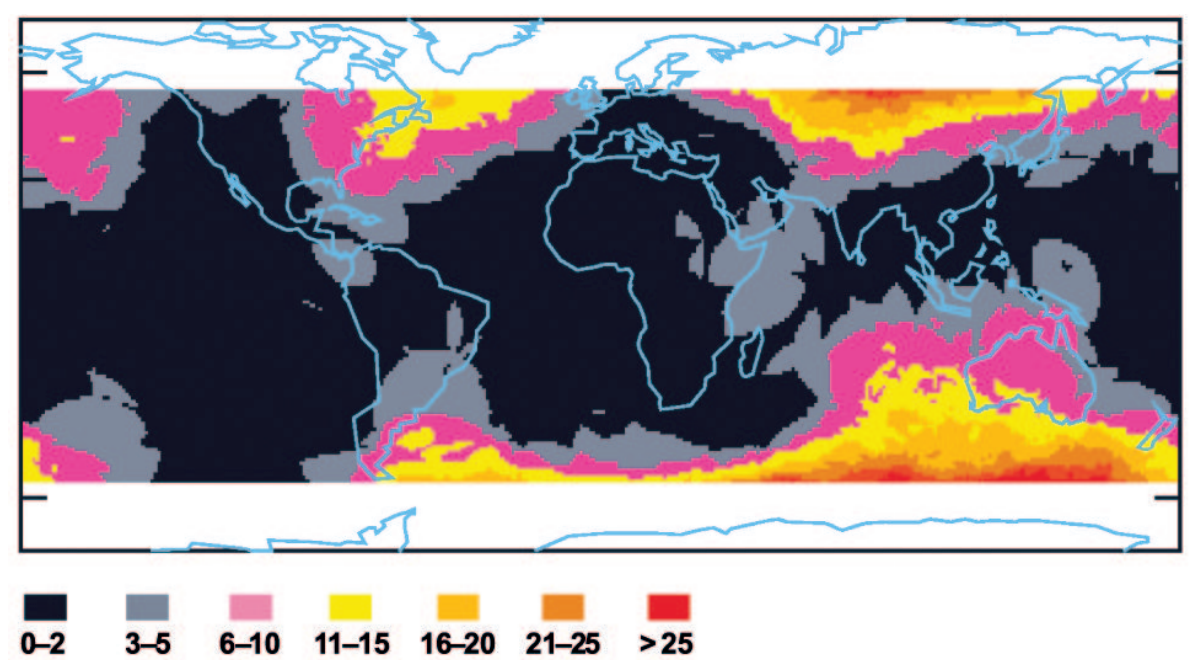

Abb. 4.3: VGP-Dichte für die Matuyama-Brunhes-Polumkehr. Jeder Punkt zwischen $55^{\circ}$ Süd und $55^{\circ}$ Nord gibt mit seiner Farbe die Anzahl der VGP's an, die in einem $15^{\circ}$-Radius um ihn herum zu finden sind. Nach Hoffman (2000).

Temperatur im untersten Bereich des Erdmantels, welche sowohl zu erhöhten seismischen Geschwindigkeiten im Mantel als auch zu einem erhöhten Wärmefluß aus dem Kern in den Mantel führen (s. Abb. 1.2). Dort, wo der untere Mantel kalt ist, wird auch die darunterliegende Flüssigkeit des äußeren Kerns im Mittel etwas kälter und deswegen schwerer sein. Das hat Auswirkungen auf die Konvektion und damit auch auf das Magnetfeld. wie von Olson \& Christensen (2002) in Dynamosimulationen demonstriert werden konnte. Der Effekt war allerdings weniger in instantanen Bildern des Geschwindigkeits- und Magnetfeldes zu sehen, sondern erst deutlich bei Mittelung über mehrere magnetische Diffusionszeiten.

Es ist nicht anzunehmen, daß die Ursache der beobachteten langzeitigen VGP-Häufungen allein im Erdkern zu finden ist, denn dafür ist die Konvektion dort viel zu schnell. Die Zeitskala der Konvektion im flüssigen Erdkern läßt sich mit einer typischen Flüssigkeitsgeschwindigkeit von $v_{\mathrm{K}}=10 \mathrm{~km} / \mathrm{Jahr}$ und der Kerndicke von $D_{\mathrm{K}}=2270 \mathrm{~km}$ abschätzen $\mathrm{zu}$

$$
t_{\mathrm{K}} \approx \frac{D_{\mathrm{K}}}{u_{\mathrm{K}}} \approx 200 \text { Jahre }
$$

Die Zeitskala der Mantelkonvektion liegt viele Größenordnungen darüber; mit $u_{\mathrm{M}} \approx 5$ cm/Jahr (Bewegung der tektonischen Platten) und $D_{M}=2900$ km erhält man:

$$
t_{\mathrm{M}} \approx \frac{D_{\mathrm{M}}}{u_{\mathrm{M}}} \approx 50000000 \text { Jahre }
$$

Wesentliche Änderungen der thermischen Struktur des Mantels benötigen viele Millionen Jahre. (Der Transport von Wärme durch Diffusion spielt im Mantel in den betrachteten Grössenordnungen keine Rolle.) Die Beständigkeit der VGP-Verteilung über mehr als 10 Mio. Jahre legt nahe, daß der Dynamoprozeß im Kern durch die Temperaturanomalien des unteren Mantels beeinflußt wird, welche über wesentlich längere Zeiträume unverändert bleiben. Ein weiterer Hinweis auf die Verbindung zwischen der thermischen Struktur des Mantels und dem Geodynamo ist die Tatsache, daß die Länge des längsten beobachteten stabilen 
Zeitintervalls, des sogenannten „Superchrons“ der Kreidezeit, mit der Zeitskala der Mantelkonvektion übereinstimmt (s. Abb. 1.1).

Ein heterogener Wärmefluß an der KMG kann neben den schon beschriebenen Auswirkungen auch einen starken Einfluß auf die Umpolfrequenz eines Dynamos haben, wie von Glatzmaier et al. (1999) gezeigt wurde. Ob der Einfluß der gleiche in einem besser aufgelösten Modell ohne Hyperdiffusivitäten ist, soll hier überprüft werden.

\subsection{Zusammenfassung thermischer und chemischer Konvektion}

Die Konvektion wurde bis jetzt entweder rein thermisch oder rein chemisch angetrieben. Im Folgenden werden Modelle benutzt, welche grundsätzlich chemisch durch das Freisetzen eines leichten Elementes an der IKG angetrieben werden, welchen aber zusätzlich noch thermische Inhomogenitäten an der KMG aufgeprägt sind. Der Wärmefluß aus dem Kern wird exakt dem adiabatischen Wärmefluß gewählt, so daß der thermische Beitrag zur Konvektion nur durch die Flußinhomogenitäten an der KMG entsteht. Wenn thermische und chemische Diffusivitäten gleich groß sind, können die Variablen $T$ und $C$ zu einer einzigen „Auftriebsvariablen“ $X$ zusammengefaßt werden. Natürlich ist der molekulare Wert der chemischen Diffusivität um einige Größenordnungen kleiner als der der thermischen. Es wird aber angenommen, daß die Diffusivitäten effektive Werte darstellen, welche bei turbulenter Durchmischung der Flüssigkeit vergleichbar groß werden.

Gehen wir von der Wärmeleitungsgleichung und der Gleichung für den Transport des leichten Elementes (s. Glg. 2.12) aus:

$$
\begin{gathered}
\frac{\partial T}{\partial t}+\mathbf{u} \cdot \nabla T=\kappa \nabla^{2} T \\
\frac{\partial C}{\partial t}+\mathbf{u} \cdot \nabla C=\kappa_{c} \nabla^{2} C-\gamma
\end{gathered}
$$

Setzt man $\kappa=\kappa_{c}$, multipliziert Glg. 4.1 mit $\alpha$ und und addiert sie zu Glg.4.2, erhält man die Gleichung für den Transport der Auftriebsvariablen $X$ :

$$
\frac{\partial X}{\partial t}+\mathbf{u} \cdot \nabla X=\kappa \nabla^{2} X-\gamma
$$

$X$ wird definiert als

$$
X=C+\alpha T
$$

und die Dichte im Auftriebsterm ist gegeben als

$$
\rho=\rho_{0}(1-C-\alpha T)=\rho_{0}(1-X)
$$

Die Skalierung von $X$ erfolgt mit dem Term $\gamma$ der internen Senken, $X=X^{\prime} \cdot \gamma D^{2} / \kappa$, wobei $X^{\prime}$ die skalierte Auftriebsvariable ist. Die skalierte Form der Gleichung für den Transport von 
$X$ entspricht Glg. 2.3 mit negativem $S$, wobei $T$ ersetzt ist durch $X$. Die Rayleighzahl Ra in der Navier-Stokes-Gleichung (2.1) wird dann ersetzt durch

$$
\mathrm{Ra}^{x}=\frac{\gamma D^{3} g_{0}}{\Omega \kappa \nu}
$$

Wie im Fall der chemischen Konvektion (Modelle Typ 8 und 9) wird der Term der internen Senken zu $S=-1$ gewählt. An der äußeren Grenze muß der Fluß im Mittel verschwinden, was bedeutet, daß gilt: $f_{0}(l=0, m=0)=0$. Die Amplitude der Flußdichte höherer harmonischer Moden kann aber ungleich Null gewählt werden. Die dimensionslose Flußdichte $f_{i}$ an der inneren Grenze wird durch die Bedingung festgelegt, daß die volumetrischen Senken $S$ sich mit dem Fluß durch die innere Grenze genau kompensieren, damit es keine zeitliche Drift von $X^{\prime}$ gibt:

$$
\oint_{\mathrm{IKG}} f_{i} \cdot d A=\int_{V} S \cdot d V
$$

daraus ergibt sich für $f_{i}$ :

$$
f_{i}=\left.\frac{\partial X^{\prime}}{\partial r^{\prime}}\right|_{r=r_{i}}=\frac{S}{3} \frac{r_{0}^{3}-r_{i}^{3}}{r_{i}^{2}}=-3.9963274
$$

Im Falle einer homogenen Flußrandbedingung an der äußeren Grenze entspricht das Modell dem Antriebstyp 9 (chemische Konvektion mit festem Fluß am inneren Kern). Aus der vorangegangenen Studie ist für den Fall $\mathrm{E}=3 \times 10^{-4}, \mathrm{q}=3$ ungefähr bekannt, bei welchen Parametern für dieses Modell Polumkehrungen zu erwarten sind (Abb. 3.7).

Im konduktiven Fall beträgt der Kontrast von $X^{\prime}$ zwischen innerer und äußerer Grenze

$$
\Delta X_{\mathrm{kond}}^{\prime}=X_{\mathrm{kond}}^{\prime}\left(r=r_{0}\right)-X_{\mathrm{kond}}^{\prime}\left(r=r_{i}\right)=1.117486
$$

Diesen Wert berechnet man durch Lösung der Transportgleichung für $X$ in Kugelkoordinaten für den stationären, konduktiven Fall. Im Folgenden wird der Strich bei $X^{\prime}$ wieder fortgelassen, aber mit $X$ soll die dimensionslose Auftriebsvariable bezeichnet sein.

\subsection{Vorgehensweise}

Die Auswirkungen des heterogenen Wärmeflusses sollen zuerst an einem Modell mit großer Ekmanzahl untersucht werden, bevor zu realistischeren Parametern übergegangen wird, die sehr viel mehr Rechenzeit erfordern. Auf einem 4-Prozessor-Knoten des benutzten Computers (einer IBM RS/6000 SP, $375 \mathrm{MHz}$ ) benötigt das parallelisierte Programm mehr als eine Woche, um eine magnetische Diffusionszeit eines umkehrenden Dynamos bei $\mathrm{E}=3 \times 10^{-4}$ $\mathrm{zu}$ berechnen, aber nur einige Stunden bei $\mathrm{E}>10^{-3}$. Es wird für die neue Antriebsart ein umkehrender Dynamo gesucht, und zwar zunächst mit homogenem Wärmefluß an der KMG. In einem zweiten Schritt wird dem Dynamo ein longitudinal variierendes Wärmeflußmuster aufgeprägt und sein Einfluß auf die Verteilung der virtuellen Pole betrachtet. Später werden verschiedene Wärmeflußmuster an Dynamos mit $\mathrm{E}=3 \times 10^{-4}$ untersucht.

In Abb.4.4 sind die acht betrachteten Wärmeflußmuster zusammengestellt. Oben links das Modell a mit homogenem (adiabatischem) Wärmefluß als Referenzmodell, rechts daneben b 
a) homogen

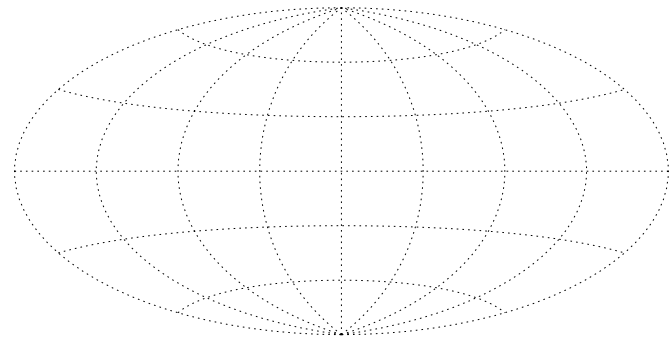

c) $l=2, m=0$

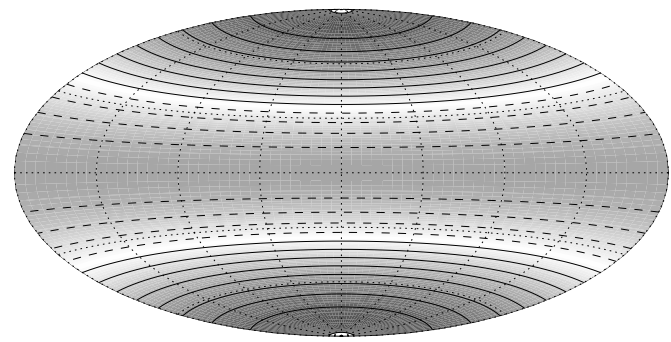

e) $l=4, m=0$

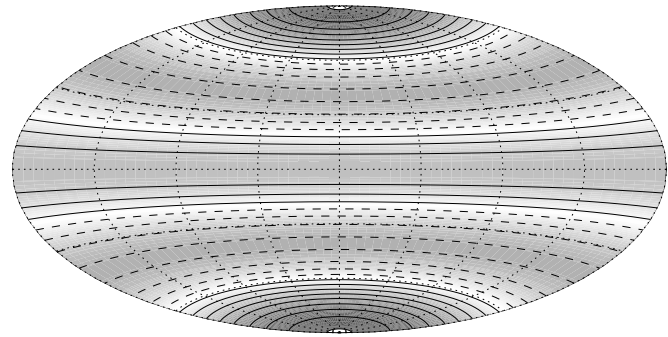

g) $l=2, m=2$

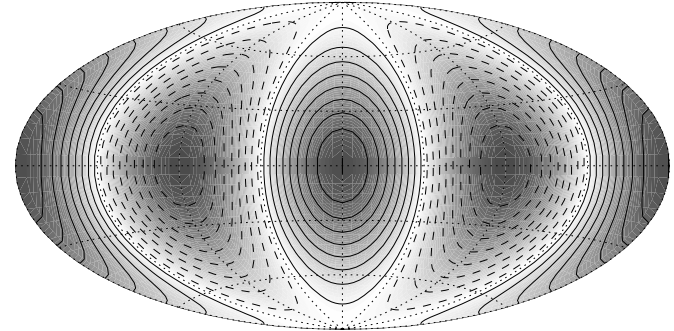

b) $l=1, m=0$

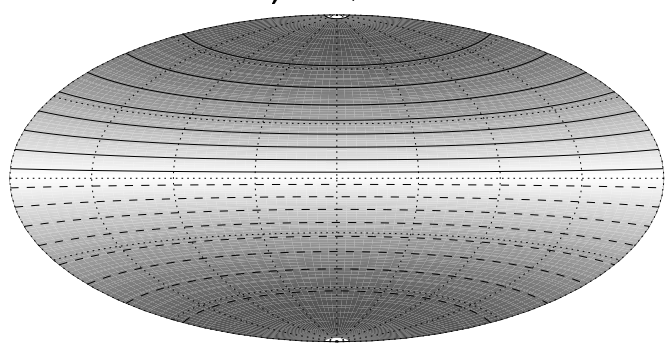

d) $\mathrm{l}=2, \mathrm{~m}=0$, invers

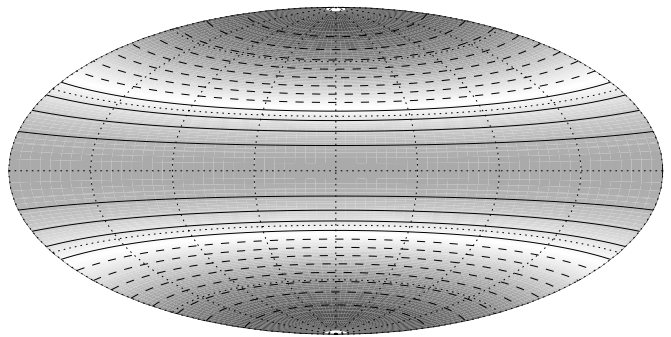

f) $l=4, m=0$, invers

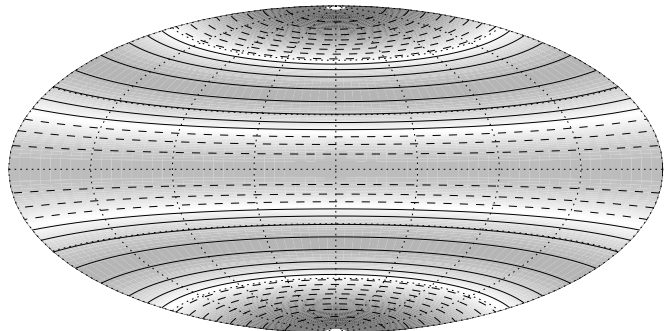

h) tomographisch

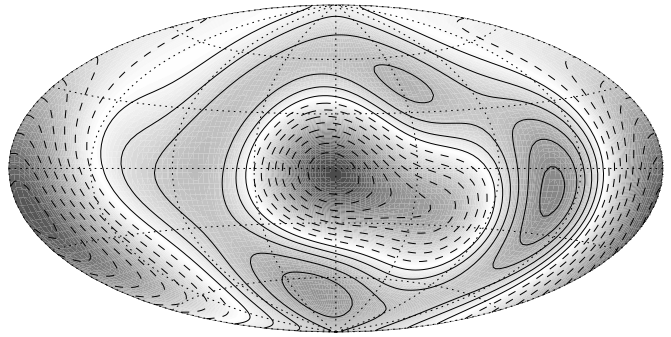

Abb. 4.4: Acht verschiedene Wärmeflußmuster an der KMG. Überadiabatischer Fluß innerhalb durchgezogener Linien, unteradiabatischer innerhalb gestrichelter. Der Betrag des Flusses ist durch den Grad der Schattierung gekennzeichnet. $\phi=\theta=0^{\circ}$ liegt im Zentrum der Projektion. 
mit einer Variation in der $(l=1, m=0)$-Mode: die Flußvariation ist rotationssymmetrisch, positiv in der Nordhemisphäre und negativ in der Südhemisphäre. Modelle c - g sind symmetrisch bezüglich des Äquators. Mit dem Paar c und d soll untersucht werden, welche Auswirkung das Vorzeichen der Flußvariation hat; e und $f$ unterscheiden sich von $c$ und $d$ durch die feineren Strukturen. Damit soll untersucht werden, ob großskalige Variationen einen anderen Einfluß haben als kleinskalige. g beschreibt eine longitudinale Variation, welche dem tomographischen Modell h schon recht ähnlich ist, wenn man das Vorzeichen umdreht. Mit h soll simuliert werden, wie der Wärmefluß tatsächlich für die Erde aussehen könnte. Es ist aus der seismischen Tomographie des unteren Mantels abgeleitet, und zwar aus einem Modell von Masters et al. (1996), welches für diese Zwecke auf die ersten vier Kugelfunktionsmoden beschränkt wurde. Es wurde angenommen, daß die Variationen der seismischen Geschwindigkeit sich umgekehrt proportional zur Temperatur verhalten. Da der Kern gegenüber dem Mantel quasi isothermal ist, übersetzt sich eine niedrige Temperatur am Boden des Mantels direkt in einen hohen Wärmefluß in den Mantel und umgekehrt. Über die Amplitude der Wärmeflußvariationen sagen die Variationen der seismischen Geschwindigkeit leider nichts aus.

Die Amplitude der Variation für die Modelle b bis g wird zu $f_{0}(l, m)=0.106$ oder zu $f_{0}(l, m)=0.176$ gewählt. Entspräche die dimensionslose Flußdichte an der äußeren Grenze überall ihrem Maximalwert von 0.106 (bzw. 0.176), so würde der Gesamtfluß durch die äußere Grenze 20\% (bzw. 35\%) des Flusses durch die innere Grenze ausmachen. Vergleicht man die Flußdichte $f_{0}(l, m)$ direkt mit der Flußdichte an der inneren Grenze, $f_{i} \approx-4.0$ (Glg. 4.8), so scheint die Variation klein zu sein. Die äußere Grenze hat allerdings eine mehr als achtmal so große Fläche wie die innere Grenze, so daß kleine Variationen in der Flußdichte einen großen Einfluß haben können: Bei einigen selbsterhaltenden Dynamos führt das Aufprägen einer Wärmeflußvariation zum Zerfall des Magnetfeldes, was der Amplitude der Variation eine natürliche (parameterabhängige) Obergrenze setzt. Bei $\mathrm{E}=10^{-2}, \mathrm{Pm}=$ $20, \mathrm{Ra}^{x}=300$ führt eine Wärmeflußvariation von $f_{0}(2,2)=0.106 \mathrm{z}$. B. zum Zerfall des Magnetfeldes, während der gleiche Dynamo mit homogenem Wärmefluß selbsterhaltend ist (s. u.). Bei $\mathrm{E}=3 \times 10^{-4}$ blieben jedoch alle untersuchten Modelle auch nach Aufprägen der Wärmeflußvariation mit $f_{0}(l, m) \leq 0.176$ selbsterhaltend. Für das tomographische Modell wird die Amplitude der am stärksten beitragenden Mode (der $l=2, m=2$-Mode) im Modell $\mathrm{H}$ zu 0.176 bzw. in h halb so groß zu 0.088 gewählt.

Um VGP's für jeden beliebigen Punkt auf der Erdoberfläche bestimmen zu können, wird das Magnetfeld der KMG unter der Annahme, daß der Mantel ein elektrischer Isolator ist, an die Erd- bzw. Modelloberfläche fortgesetzt und dort in Form von Gauß-Koeffizienten bis Grad und Ordnung acht abgespeichert. Für die Skalierung auf die Erde werden die folgenden Werte benutzt: mittlere Kerndichte $\rho=1.1 \cdot 10^{4} \mathrm{~kg} / \mathrm{m}^{3}$, Leitfähigkeit des Kern $\sigma=6 \cdot 10^{5} \mathrm{~S} / \mathrm{m}$, Radien für Erde, Kern und inneren Kern $r_{E}=6371 \mathrm{~km}, r_{K}=3485 \mathrm{~km}$ und $r_{I}=1215 \mathrm{~km}$, Winkelgeschwindigkeit $\Omega=7.29 \cdot 10^{-5} \mathrm{~s}^{-1}$. Durch die Feldfortsetzung an die Oberfläche (Verhältnis Erdradius zu Kernradius ist etwa $r_{E} / r_{0}=1.8$ ) werden die kleinskaligen Magnetfeldstrukturen abgeschwächt und zwar um den Faktor $\left(r_{0} / r_{E}\right)^{l+2}$. Da die höheren Harmonischen zum Oberflächenfeld nur einen vernachlässigbar kleinen Beitrag liefern, genügt die Beschränkung auf die ersten acht Kugelfunktionsgrade. Die dadurch recht geringe Datenmenge von jeweils 88 Koeffizienten ermöglicht es, das Feld in kurzen Zeitabständen zu speichern. Typischerweise geschieht dies alle 1000 Zeitschritte, was größenord- 


\begin{tabular}{rcccrrccc}
\hline $\mathrm{Ra}^{x}$ & $\mathrm{q}$ & $\mathrm{Rm}$ & $\mathrm{Nu}$ & $\bar{E}_{\mathrm{mag}}$ & $\bar{E}_{\mathrm{mag}}^{\ell=1}$ & $\bar{B}_{\mathrm{KMG}}$ & $\bar{B}_{\text {dip }}$ & $t_{r}$ \\
\hline 200 & 20 & 206 & 1.48 & - & - & - & - & - \\
250 & 10 & 119 & 1.54 & - & - & - & - & - \\
250 & 13 & 155 & 1.55 & 2.2 & $9.9 \%$ & 0.064 & 0.034 & 0.15 \\
250 & 15 & 178 & 1.55 & 3.9 & $8.3 \%$ & 0.090 & 0.046 & 0.14 \\
250 & 20 & 238 & 1.56 & 6.2 & $8.7 \%$ & 0.108 & 0.054 & 0.13 \\
300 & 20 & 263 & 1.66 & 19.7 & $17.1 \%$ & 0.223 & 0.103 & 0.09 \\
\hline
\end{tabular}

Tab. 4.1: Dynamodaten für $\mathrm{E}=10^{-2}$, homogener KMG-Wärmefluß, Die Nusseltzahl Nu ist analog zu Kap. 3 definiert als $\Delta X_{\text {kond }} / \Delta X_{\text {konv }}$ mit $\Delta X_{\text {kond }}$ wie in Glg. 4.9 gegeben. Strich (-): das Magnetfeld zerfällt mit der Zeit. Alle Dynamo-Lösungen zeigen periodische Umkehrungen des Dipolfeldes, $t_{r}$ gibt die Zeitspanne an, in der der Dynamo jeweils in einer Polarität ist.

nungsmäßig 1000 Koeffizientensätze pro magnetische Diffusionszeit ausmacht. Mit diesen Daten kann dann für einen beliebigen Ort an der Oberfläche $D$ und $I$ und damit der VGP berechnet werden. Dies wird zu einem Zeitpunkt nicht nur für einen Ort gemacht, sondern für viele über den Globus verteilte Sites, damit sich eventuell vorhandene ortsabhängige Effekte herausmitteln.

\subsection{Voruntersuchung bei großer Ekmanzahl}

Bei der Ekmanzahl $\mathrm{E}=2 \times 10^{-2}$ und der Robertszahl $\mathrm{q}=10$ konnten für verschiedene Rayleighzahlen $\mathrm{Ra}^{x}$ keinen selbsterhaltenden Dynamos gefunden werden. Die Magnetfelder wechselten zwar ihre Polarität, wurden dabei aber immer schwächer. Im Regime dipoldominierter Dynamos (vgl. Abb. 3.5) bleibt das Magnetfeld, auch wenn es zerfällt, in einer gegebenen Polarität. Offensichtlich war die Rayleighzahl hoch genug für Umkehrungen, aber die Robertszahl zu niedrig (bzw. die magnetische Diffusion zu hoch) für selbsterhaltende Dynamo-Aktion.

\subsubsection{Ein periodisch umkehrender Dynamo}

Bei $\mathrm{E}=10^{-2}, \mathrm{Pm}=20, \mathrm{Ra}^{x}=300$ wurde ein periodisch umkehrender Dynamo gefunden, der im Gegensatz zu dem aus Kap. 3.1 einen hohen Dipolbeitrag zum KMG-Feld besitzt: Der Dipol macht hier im Zeitmittel etwa die Hälfte der rms-Gesamtfeldstärke aus. Ähnliche Lösungen existieren in einem Bereich von Rayleigh- und Robertszahlen; einige charakteristische Daten sind in Tab. 4.1 zusammengestellt.

Abb. 4.5 zeigt für diesen Dynamo einen Ausschnitt aus der Zeitreihe. Gezeigt ist die mittlere kinetische und magnetische Energiedichte im Volumen der Kugelschale, $E_{\text {kin }}$ und $E_{\text {mag }}$. Wie bei allen umkehrenden Dynamos aus der Parameterstudie ist die magnetische Energie niedriger als die kinetische. In Abb. 4.6 ist die Dipolneigung sowie die rms-Gesamt- und Dipolfeldstärke an der KMG gezeigt. Der axiale Dipol wechselt etwa alle 0.09 magnetischen Diffusionszeiten sein Vorzeichen; die Geschwindigkeit der Oszillation ist parameterabhän- 


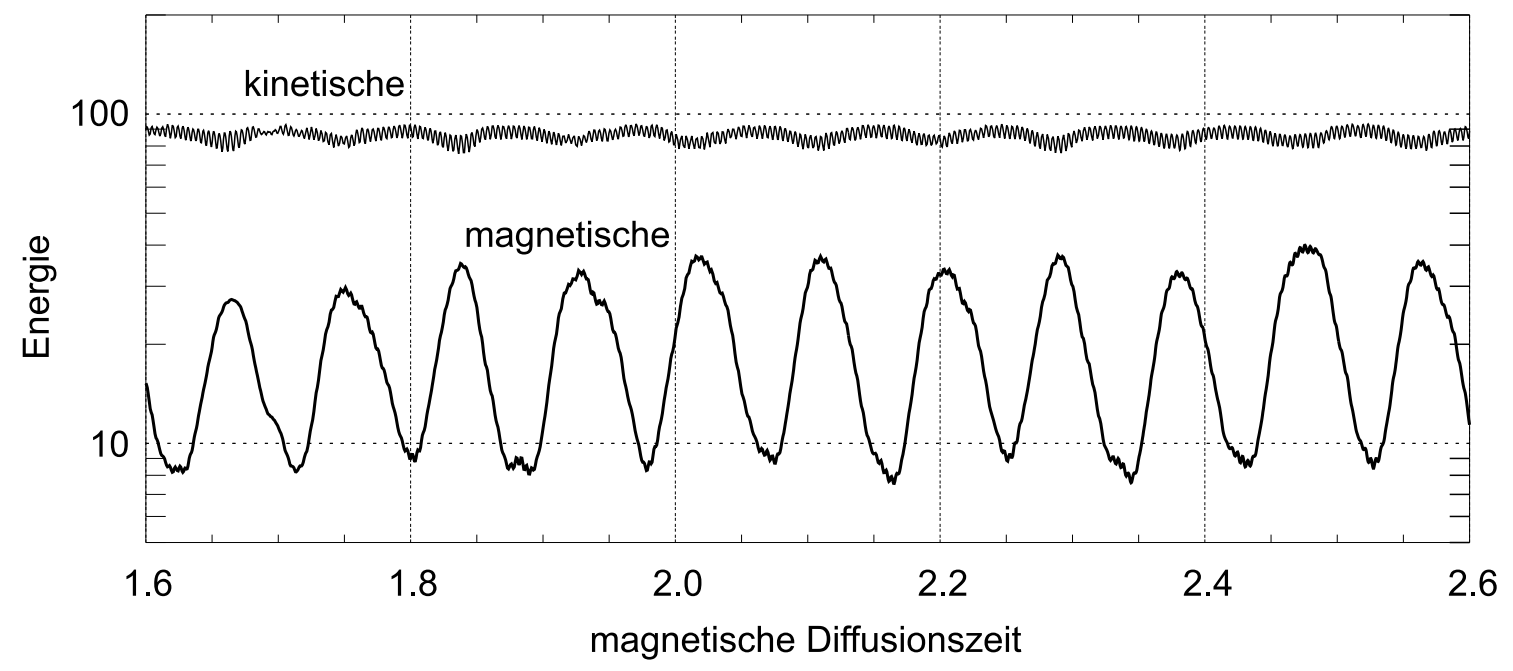

Abb. 4.5: Zeitreihe der kinetischen und magnetischen Energiedichte des periodisch umkehrenden Dynamos bei $\mathrm{E}=10^{-2}, \mathrm{Pm}=20, \mathrm{Ra}^{x}=300$.

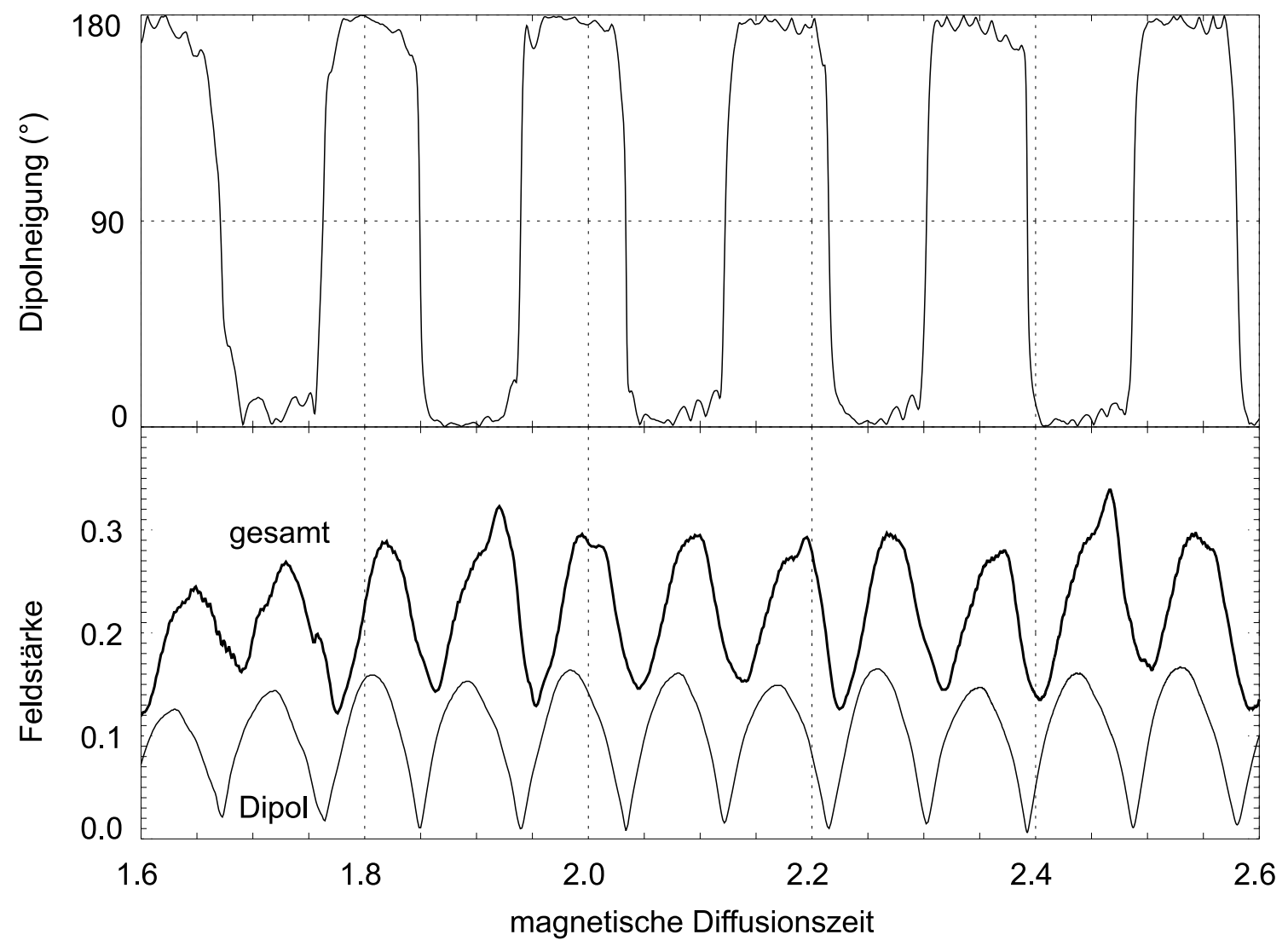

Abb. 4.6: Zeitreihe der Neigung der Dipolachse (oben) und der Feldstärke an der KMG (unten) des Dynamos aus Abb. 4.5 
gig, wie man aus Tab. 4.1 entnehmen kann. Die gleiche Periodizität ist in der magnetischen Energie zu erkennen, die ihr Maximum jeweils kurz vor dem bevorstehenden Umkippen der Dipolachse erreicht. Während die magnetische Energie hoch ist, ist die kinetische leicht reduziert. Der kinetischen Energie ist zusätzlich eine kurzperiodische Schwingung überlagert, welche aber auch im Fall ohne Magnetfeld vorhanden ist; sie ist also eine Eigenschaft der Konvektion. Aus der Zeitreihe der Feldstärken an der KMG ist zu erkennen, daß die Dipolfeldstärke fast auf Null absinkt, wenn die Dipolneigung um $90^{\circ}$ liegt. Es gibt während der Umkehr also keinen nennenswerten Beitrag des äquatorialen Dipols; der Gesamt-Dipol kippt bei einer Umkehr nicht einfach in die andere Lage, sondern er baut sich ab und darauf in der umgekehrten Richtung wieder auf. Die Dipolfeldstärke an der KMG erreicht jeweils in der Mitte zwischen zwei Umkehrungen ihren Maximalwert, während das Maximum der Gesamtfeldstärke erst etwas später folgt, nämlich kurz vor der nächsten Umkehr.

Abb. 4.7 zeigt die Strömungsstruktur dieses Dynamos. Am Nordpol gibt es einen Aufstrom und bei etwa $20^{\circ}$ Breite zwei diametral gegenüberliegende Abströme, wie in a) und c) zu sehen ist. Auf der Südhalbkugel (Bild c) gibt es einen leichten Abstrom direkt am Pol und zwei starke, sich diametral gegenüberliegende Aufströme bei etwa $45^{\circ}$ Breite. Dem ganzen überlagert sich eine starke mittlere Strömung in prograder Richtung in der Nordhemisphäre und in retrograder Richtung in der Südhemisphäre (Bild b), welche die beiden Aufströme im Süden mit sich zieht. Der zeitgemittelte toroidale, axialsymmetrische Anteil der kinetischen Energie beträgt 64\%, der poloidale, axialsymmetrische $15 \%$. Die poloidalen Stromlinien zeigen eine Abwärtsbewegung des Materials in der Nähe des Tangentialzylinders und eine Aufwärtsbewegung im äußeren Bereich des Kerns zwischen $-45^{\circ}$ und $+45^{\circ}$ Breite. Als Tangentialzylinder bezeichnet man den imaginären, parallel zur Rotationsachse ausgerichteten Zylinder, welcher in der Äquatorebene genau den inneren Kern berührt. Zwar zeigt Abb. 4.7nur einen Schnappschuß der Strömung, diese variiert aber bis auf die Drift der Auf- und Abströme zeitlich kaum.

Wie eine Polumkehr bei diesem Dynamo abläuft, ist in Abb. 4.8 gezeigt. Das Magnetfeld an der KMG zeigt eine starke Nord-Süd Asymmetrie, die sich schon im Geschwindigkeitsfeld angedeutet hat. In Zeiten stabiler Polarität, Bild a) und d), gibt es in der Nordhemisphäre einen zirkumpolaren Ring starker Flußkonzentration bei etwa $20^{\circ}$ Breite. Dieser liegt genau im Bereich der beiden in $\phi$-Richtung ausgedehnten Abströme (Abb. 4.7 c), welche die magnetischen Feldlinien konzentrieren. Die Bewegung der Abströme in prograde Richtung führt dazu, daß Magnetfeld an immer anderen Längen gesammelt wird, so daß sich wegen der langsamen Diffusion des Feldes eine Ringstruktur ergibt. In der Südhemisphäre gibt es eine starke Konzentration des Flusses direkt am Pol und einige verstreute Flußflecken, die mit der dort retrograd gerichteten Strömung (vgl. Abb. 4.7 b) schnell westwärts wandern.

Obwohl die Polumkehrungen bei diesem Dynamo in regelmäßiger Weise ablaufen und obwohl das Magnetfeld nicht so kleinskalig ist, wie in Abb. 3.13, konnten auch diese Umkehrungen noch nicht auf einen einfachen Mechanismus zurückgeführt werden.

Eine interessante Feststellung läßt sich allerdings machen, wenn man für diesen Dynamo die VGP's berechnet. Da das KMG-Magnetfeld jeweils zwischen zwei Polaritätswechseln vom Dipolterm beherrscht wird, ist zu erwarten, daß die VGP's dann in Polnähe liegen, also ungefähr mit dem Durchstoßpunkt der Dipolachse zusammenfallen. Wir wissen aber bereits, daß das Feld während der Umpolung nicht-dipolar ist, wodurch die VGP's ortsabhängig werden. Wie das für diesen Fall aussieht, ist in Abb. 4.9 gezeigt. Für drei Sites am Äquator 
a) Auftriebsvariable $X$

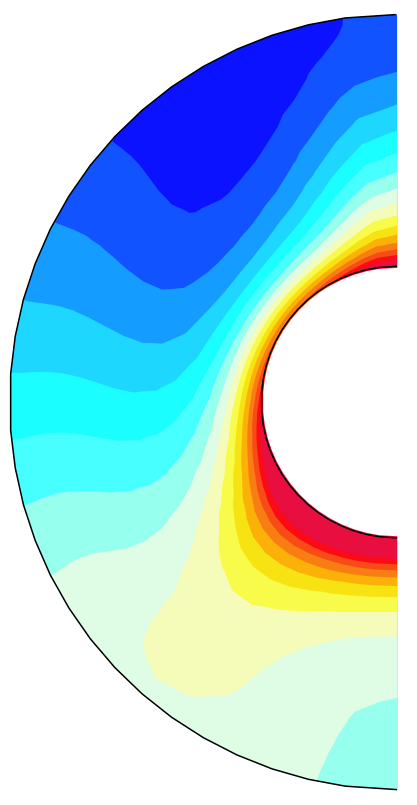

c) Radialgeschwindigkeit

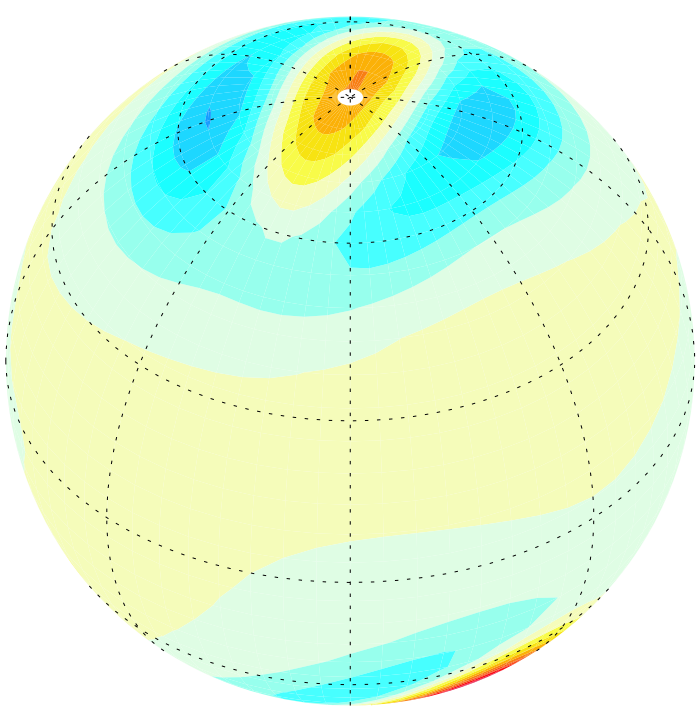

b) Mittlere Strömung

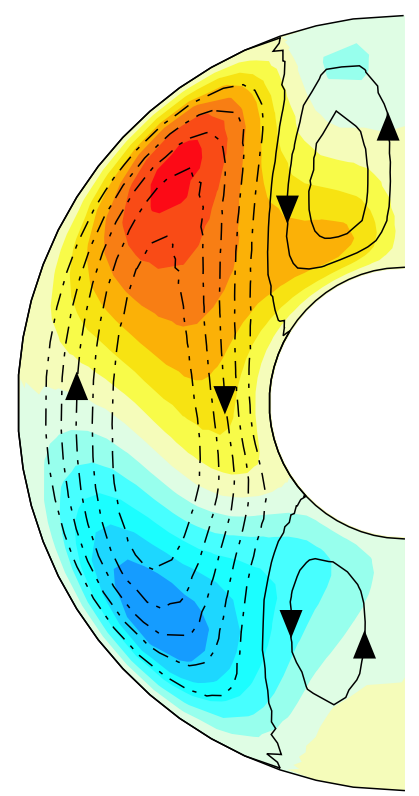

d) Radialgeschwindigkeit

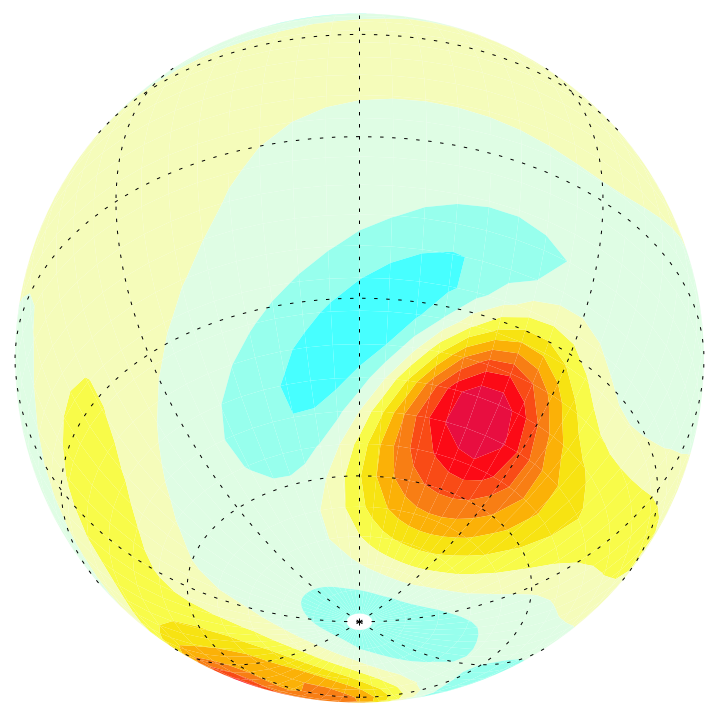

Abb. 4.7: Periodisch umkehrender Dynamo, $\mathrm{E}=10^{-2}, \mathrm{Pm}=20, \mathrm{Ra}^{x}=300$. a) Longitudinal gemitteltes $X$ im Vertikalschnitt, große (kleine) Werte von $X$ entsprechen viel (wenig) Auftrieb und roten (blauen) Farbtönen. b) Axialsymmetrischer Anteil der Strömung. Poloidaler Teil durch Stromlinien dargestellt (Umlaufsinn in Pfeilrichtung). Toroidaler Teil farblich gekennzeichnet: Rottöne (Blautöne) prograde (retrograde) Strömung. c) Radialgeschwindigkeit bei $r=0.905 r_{0}$, Blick schräg von oben. Rottöne positives, Blautöne negatives $v_{r}$. d) wie c), aber Blick von schräg unten. 


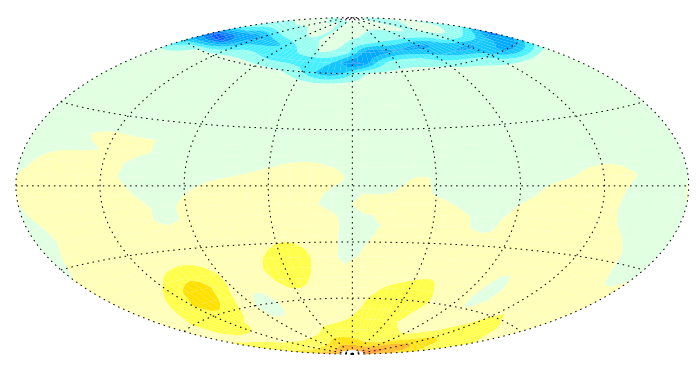

a)

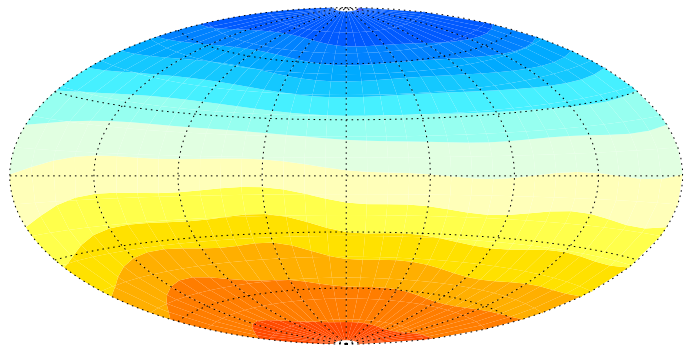

b)

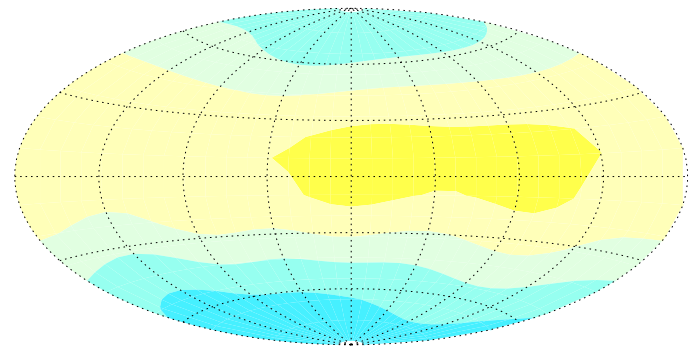

c)
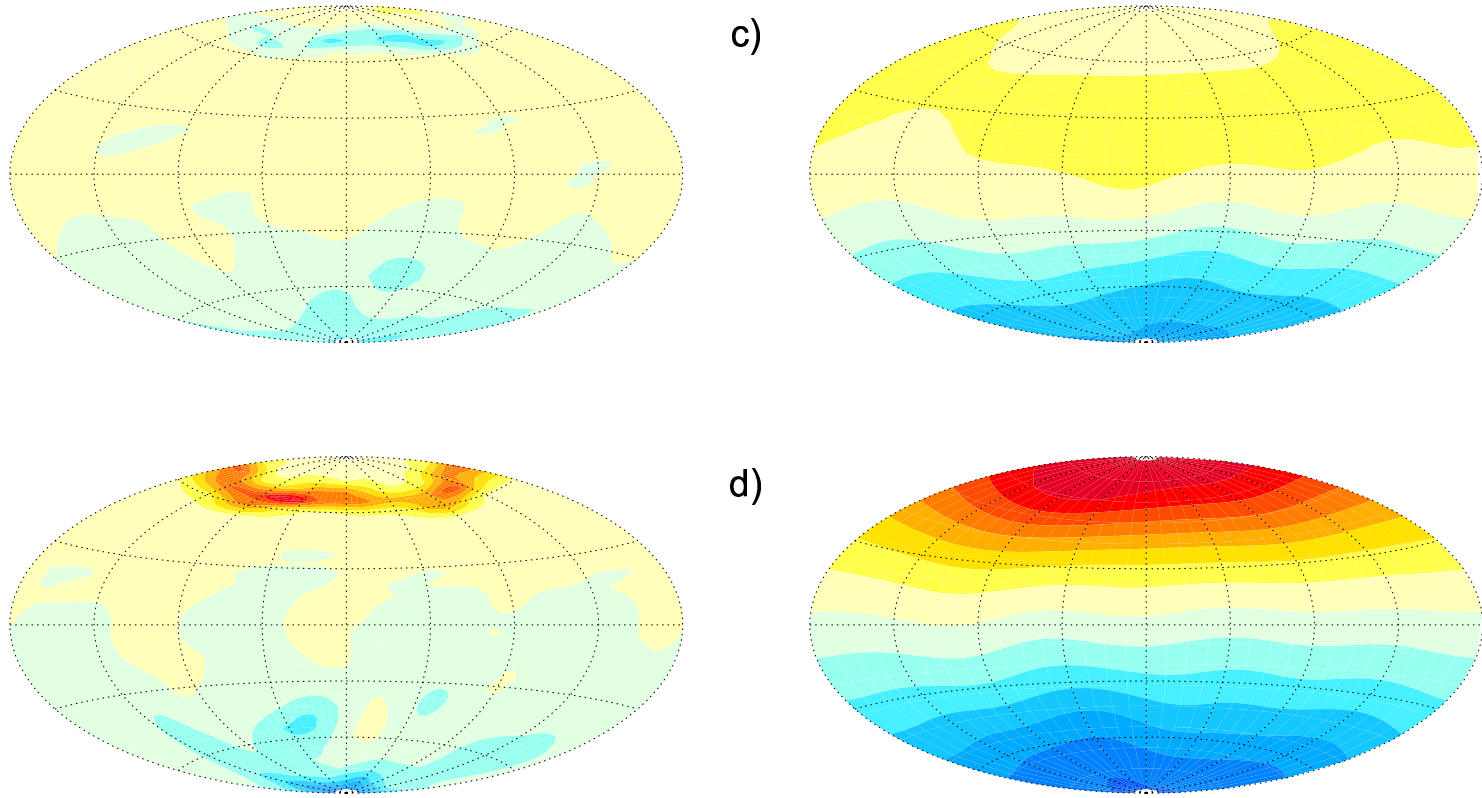

d)

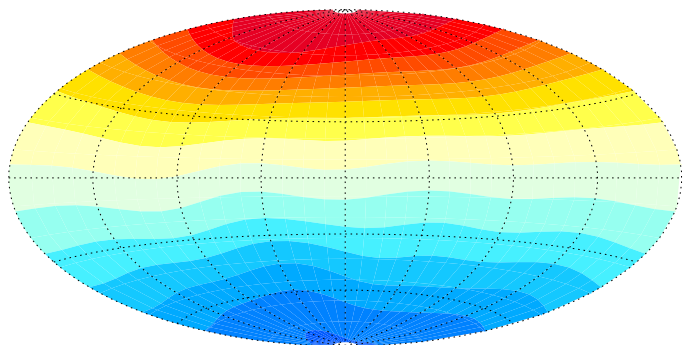

Abb. 4.8: Radialkomponente $B_{r}$ des Magnetfeldes des periodisch umpolenden Dynamos aus Abb. 4.7. Links Feld an KMG, ein Konturschritt entspricht einem $\Delta B_{r}$ von 0.19, rechts Feld an der Oberfläche, Konturschritt 0.0067. Schnappschüsse zu magnetischen Diffusionszeiten $t=2.342$ (a), $t=2.395$ (b), $t=2.402$ (c), $t=2.436$ (d). Zeiten konsistent mit Abb. 4.6 


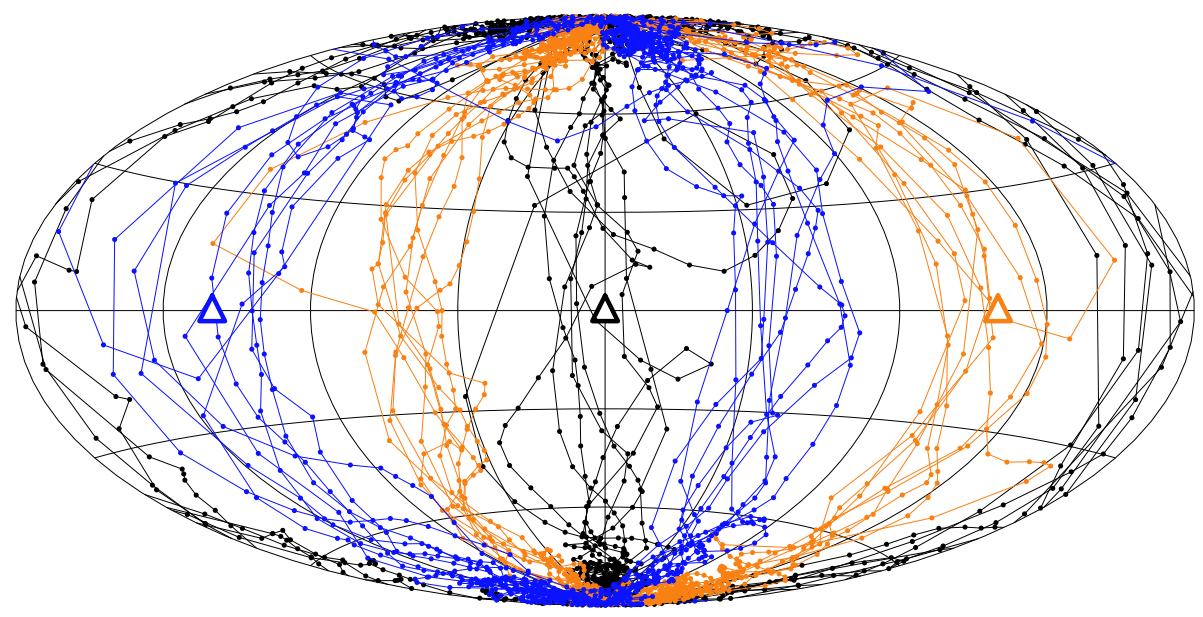

Abb. 4.9: Lage der VGP's (Punkte) für einen 2.6 magnetische Diffusionszeiten langen Zeitabschnitt des periodisch umkehrenden Dynamos aus Abb. 4.6 in HammerProjektion. Pyramiden kennzeichnen die drei Orte am Äquator, für die die VGP's berechnet wurden, diese sind farblich zugeordnet: Länge $0^{\circ}$ (schwarz), $120^{\circ}$ (orange) und $240^{\circ}$ (blau). Zeitlich aufeinanderfolgende VGP's sind durch Linien miteinander verbunden.

sind die Pfade gezeichnet, die die VGP's in diesem Zeitraum nehmen. Die schwarze Site bei $0^{\circ}$ (in der Mitte der Projektion) sieht VGP's, die bei Längen von $0^{\circ}$ oder $180^{\circ}$ den Äquator überqueren. Auch bei den beiden anderen Sites laufen die VGP's entweder ungefähr durch die Länge der Site, oder durch etwa entgegengesetzte Längengrade. Das liegt daran, daß das Magnetfeld während der Umpolung ein quadrupolares Stadium durchläuft (Abb. 4.8 b). Währenddessen ist das Feld in äquatornähe radial nach außen gerichtet (gelber Bereich in der Abbildung), so daß die Inklination dort etwa $-90^{\circ}$ beträgt. Eine Inklination von $-90^{\circ}$ bedeutet, daß der VGP genau antipodal liegt. Die VGP-Pfade auf der entgegengesetzten Seite der Sites ergeben sich, wenn das Feld aus der inversen in die normale Polarität zurück wechselt. Der Ablauf ist derselbe, nur daß $\mathbf{B}$ und $-\mathbf{B}$ vertauscht sind, was eine äquatornahe Inklination von $+90^{\circ}$ während der Umpolung ergibt, so daß die Sites den virtuellen Pol genau bei sich sehen.

\subsubsection{Ortsabhängiger Wärmefluß bei moderaten Parametern}

Wir nehmen den periodisch umkehrenden Dynamo aus dem letzten Abschnitt und prägen ihm an der äußeren Grenze einen Wärmefluß der Mode $(\ell=2, m=2)$ und der Amplitude $f_{0}(2,2)=0.106$ auf. Dadurch hören die periodischen Umpolungen zunächst auf und es etabliert sich für zwei Diffusionszeiten ein stabiler, dipol-dominierter Dynamo. Dann wird das Feld schwächer und es setzen wieder Umpolungen ein (Abb. 4.10). Der Dipolanteil am KMG-Feld ist in der umpolenden Phase zwar kleiner als in der stabilen, allerdings kann er zeitweise Werte bis 50\% erreichen (getrichelte Linie der oberen Abbildung). Im weiteren Verlauf der Zeitentwicklung zerfällt das Feld jedoch schließlich. Mehrere Versuche, einem Dynamo im Bereich der Rayleighzahlen von $\mathrm{Ra}^{x}=300 \ldots 2000$ bei $\mathrm{E}=2 \times 10^{-2}$ und $\mathrm{q}=10$ 


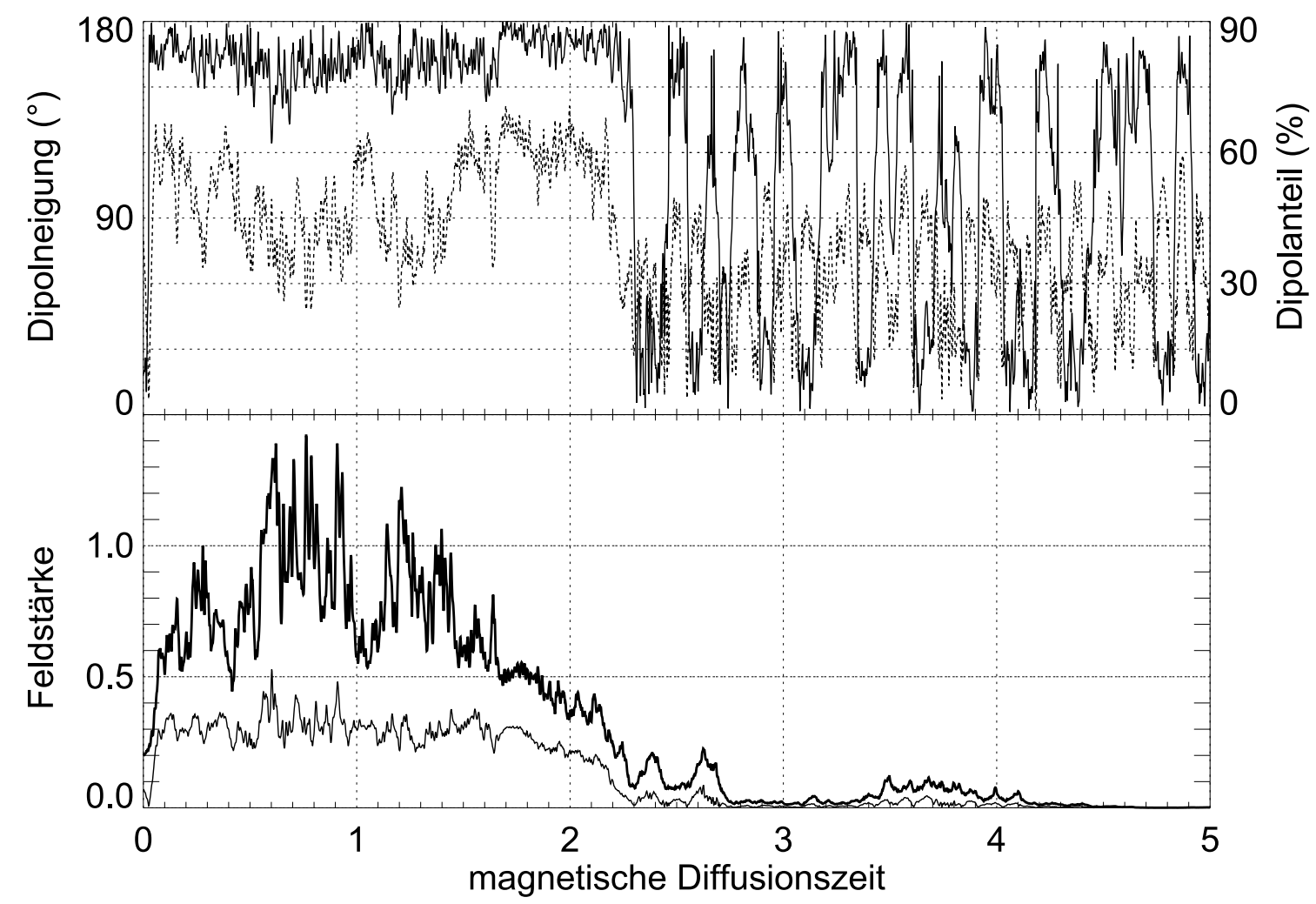

Abb. 4.10: Zeitreihe des Dynamos aus Abb. 4.5, diesmal aber mit aufgeprägtem KMGWärmefluß in der Mode $(\ell=2, m=2)$. Oben: Neigung der Dipolachse (durchgezogene Linie, linke Skala) und Anteil des Dipolfeldes am rms-Feld der ersten 11 harmonischen Moden (gestrichelt, rechte Skala); Unten: Gesamtfeldstärke an der KMG (dicke Linie) und Dipolfeldstärke (dünne Linie).

einen inhomogenen Wärmefluß aufzuprägen, führten zwar zu Umpolungen, aber gleichzeitig zum allmählichen Zerfall des Magnetfeldes. Daher wird zu kleineren Ekmanzahlen übergegangen, bei denen die Dynamos auch bei geänderten Randbedingungen weiterhin selbsterhaltend bleiben.

Bei den Parametern $\mathrm{E}=10^{-3}, \mathrm{Ra}^{x}=2000$ und $\mathrm{q}=5$ konnte ein chaotisch umkehrender Dynamo mit inhomogenem KMG-Wärmefluß gefunden werden; die Modulation ist die aus Abb. $4.4 \mathrm{~g}$ ) mit einer Amplitude von $f_{0}(2,2)=0.106$. Das Magnetfeld dieses Dynamos kehrt häufig um und zeigt viele Exkursionen (Abb. 4.11 oben). Obwohl dieser Dynamo in verschiedener Hinsicht nicht so erdähnlich ist, wie die aus Kap. 3.5, ist er besser für die Voruntersuchung geeignet, da er häufigere Umkehrungen und Exkursionen aufweist.

An der KMG beträgt die rms-Dipolfeldstärke im Zeitmittel nur 14\% der Gesamtfeldstärke (Abb. 4.11 unten), das Oberflächenfeld wird aber im Mittel vom Dipolterm dominiert, wie in Abb. 4.12 zu erkennen ist.

Zur Bestimmung des gezeigten mittleren Oberflächenspektrums wird die aus $N+1$ Werten bestehende Zeitreihe der Gauß-Koeffizienten $\left(g_{\ell}^{m}, h_{\ell}^{m}\right)_{i}, i \in 1 \ldots N$ gemittelt, indem jeder Koeffizient zum Zeitpunkt $t_{i}$ mit der (variablen) Zeitschrittlänge $\left(t_{i+1}-t_{i-1}\right) / 2$ multipliziert wird. (Die Koeffizienten für $t_{0}$ werden mit $\left(t_{1}-t_{0}\right) / 2$ gewichtet, die für $t_{N+1}$ entsprechend.) 


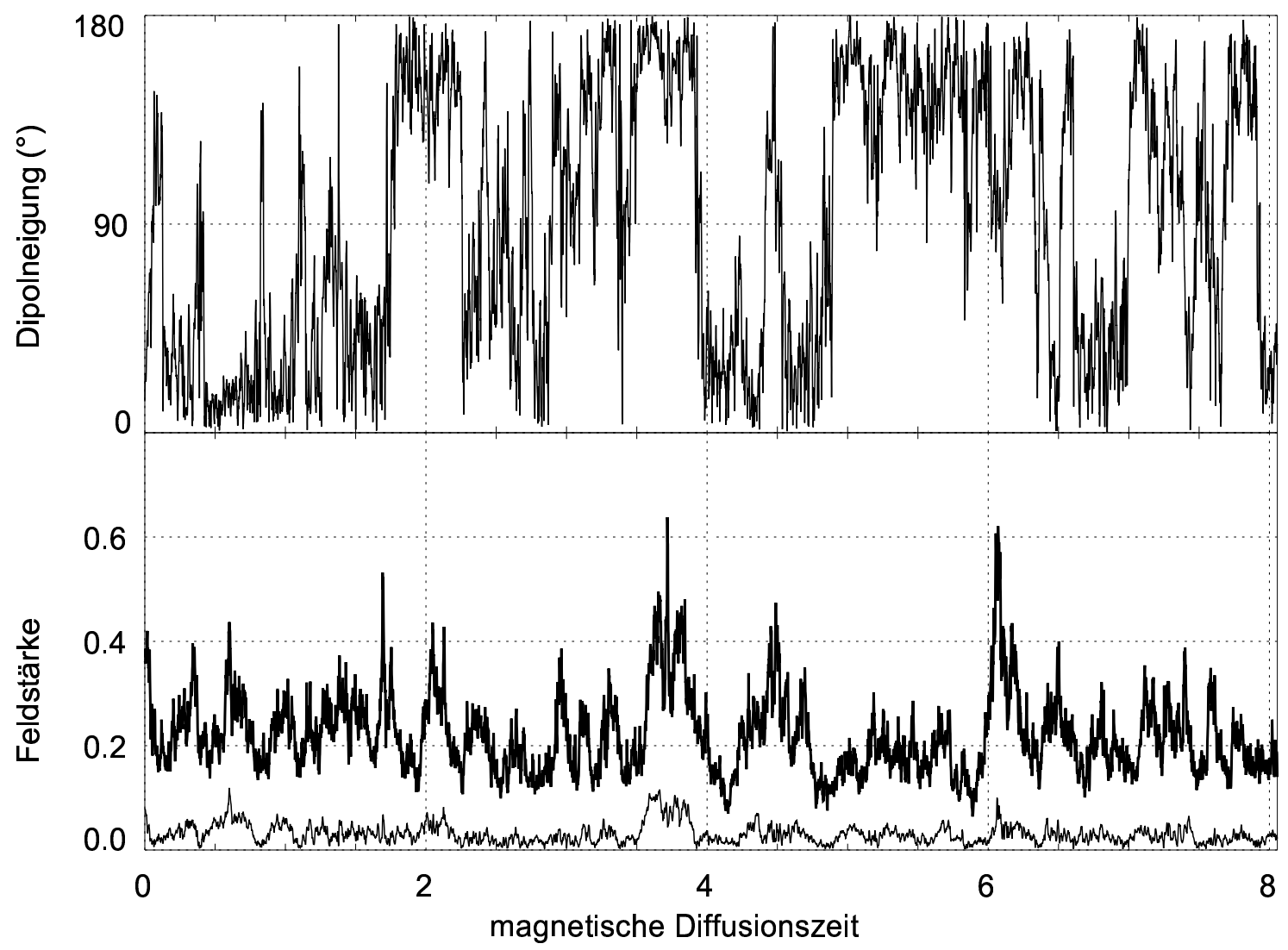

Abb. 4.11: $\mathrm{E}=10^{-3}, \mathrm{Ra}^{x}=2000, \mathrm{q}=5, f_{0}(2,2)=0.106$. Zeitreihe der Neigung der Dipolachse (oben) und der Feldstärke an der KMG (unten).Die dicke Linie gibt die Gesamtfeldstärke an, die dünne nur die Dipolfeldstärke. 


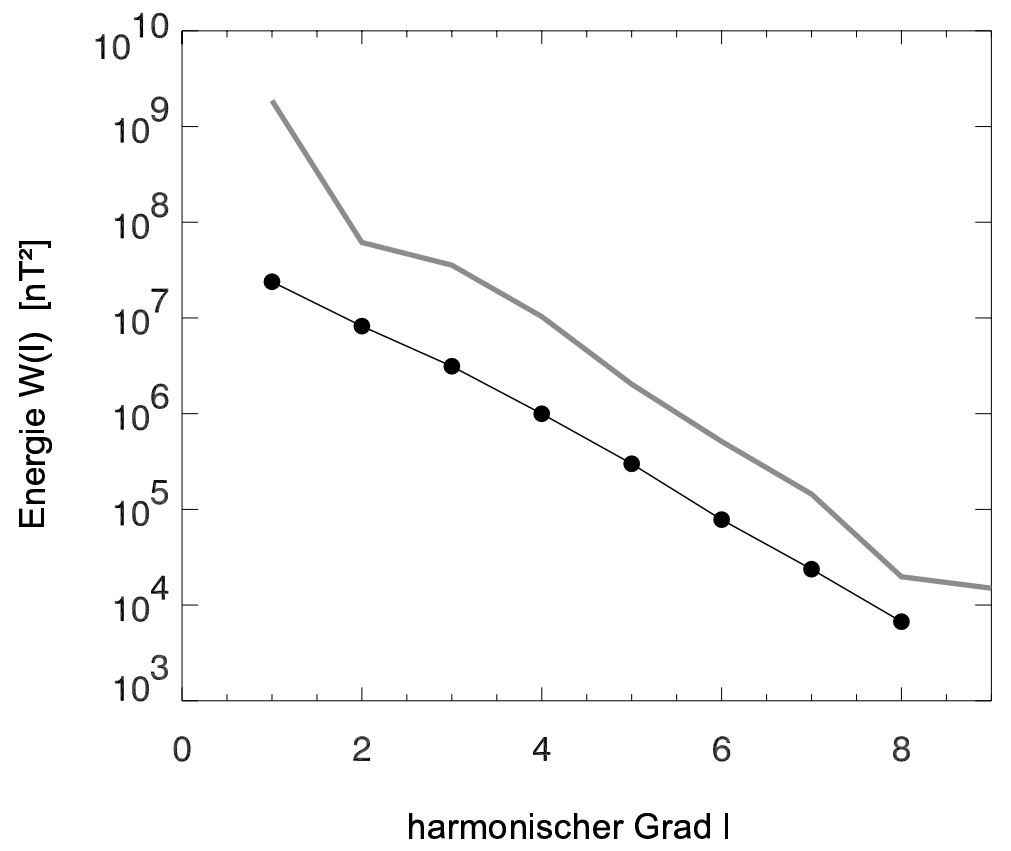

Abb. 4.12: Über 8 magnetische Diffusionszeiten gemitteltes Energiespektrum des Dynamos aus Abb. 4.11 an der Oberfläche (schwarz, Punkte). Grau zum Vergleich das Spektrum des Erdmagnetfeldes 1980 (DGRF-Daten aus Jacobs (1994))

Dann werden die Beträge aller Koeffizienten mit gleichem $\ell$ und $m$ summiert und durch die Länge der gesamten Zeitintervalls $t_{N}-t_{0}$ geteilt. Das Spektrum $W(\ell)$ ist gegeben als (Langel \& Estes. 1982)

$$
W(\ell)=(\ell+1) \sum_{m=0}^{\ell}\left(\left(g_{\ell}^{m}\right)^{2}+\left(h_{\ell}^{m}\right)^{2}\right)
$$

In die obige Formel werden dann die mittleren Koeffizienten eingesetzt. Das modellierte Feld ist zwar schwächer als das Erdmagnetfeld (was keine Auswirkungen auf die Lage der VGP's hat), aber das mittlere Spektrum der Simulation zeigt qualitativ einen ähnlichen Verlauf wie das heutige Spektrum des Erdfeldes. Letzteres hat einen höheren Dipolanteil, ist aber auch ein instantanes Spektrum. Der Dipolanteil kann in den Modellen während einer Umpolung sehr klein werden (Abb. 3.15), und es ist anzunehmen, daß das auch auf die Erde zutrifft. Es wurde ein Modell für den Geodynamo in Zeiten von Umpolungen gefunden, für das jetzt die Verteilung der virtuellen Pole betrachtet wird.

Die VGP's werden für 200 Sites bestimmt, die in einem regelmäßigen Gitter auf dem Globus verteilt liegen (Abb. 4.13). Die hohe Anzahl an Orten, für die VGP's bestimmt werden, ist gewählt worden, um ortsabhängige Einflüsse auf ein Minimum zu reduzieren. Diese können entstehen, wenn man VGP's nur für einige ausgewählte Koordinaten bestimmt; hier soll es aber zunächst um globale Effekte gehen. Im Paläomagnetismus ist man natürlich auf die Sites beschränkt, an denen es Vulkanismus gibt (große Teile der paläomagnetischen Daten stammen von Hawaii und Island) oder an denen Bohrungen gemacht wurden. Clement (1991) kann für seine Analyse der Matuyama-Brunhes-Umkehr z. B. auf etwa 20 Sites zurückgreifen (s. Abb.4.2). 


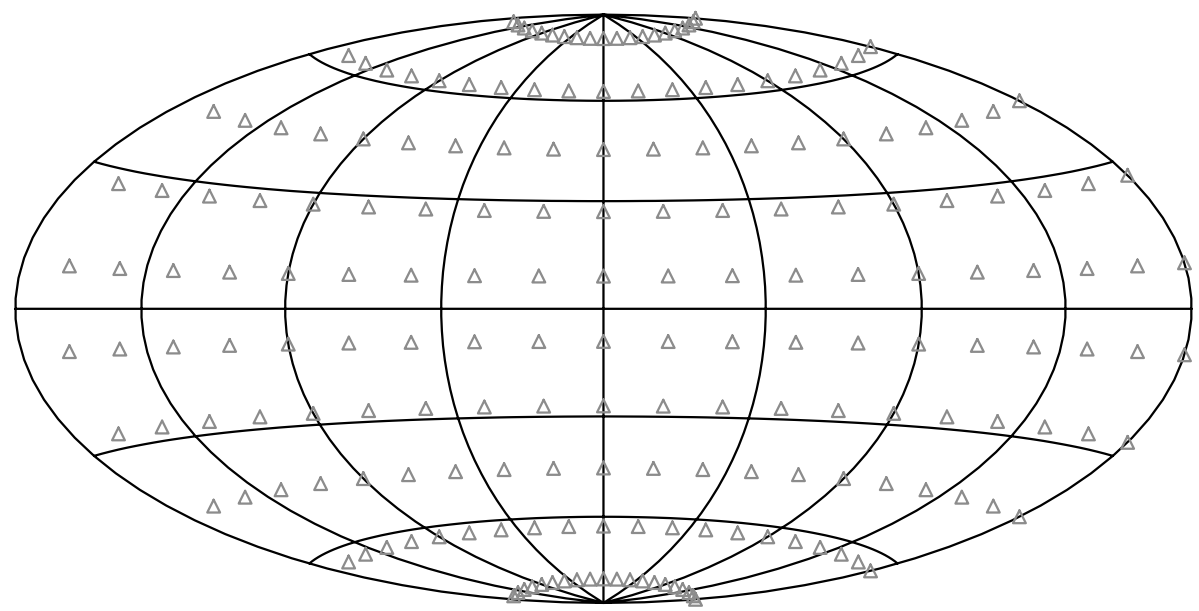

Abb. 4.13: Hammer-Projektion der Erdoberfläche mit $10 \times 20$ regelmäßig darüber verteilten Sites (Pyramiden) für welche die VGP's bestimmt werden.

Für die Analyse werden die VGP's in $1^{\circ}$-Abschnitte entlang der Breitengrade einsortiert und summiert. Die Summation erfolgt zeitgewichtet (s. Seite 59), da die Zeitabstände $\Delta t$, in denen die Gauß-Koeffizienten geschrieben werden, aufgrund der variablen Zeitschrittlänge $\delta t$ des Programms nicht äquidistant sind. Die Auftragung dieser Summation gegen die 180 Breitenintervalle ist in Abb. 4.14 oben zu sehen. Dabei wurde die Kurve so normiert, daß die Summe der Werte über alle Breitenintervalle 1.0 ergibt, so daß die Kurve genau die Wahrscheinlichkeit $P$ für einen VGP angibt, in das betrachtete Breitenfenster zu fallen. Dies ergibt einen ähnlichen Verlauf, wie in den Histogrammen auf der linken Seite von Abb. 3.10 oder 3.9, mit dem Unterschied, daß bei letzteren die Breite des Durchstoßpunktes der wahren Dipolachse betrachtet wurde. Interessanterweise gibt die aus den Breiten der virtuellen Pole berechnete Dispersionskurve recht genau die Dispersionskurve des Dipolterms wieder, wie in Abb. 4.15 zu sehen ist. Die Wahrscheinlichkeitsfunktion der VGP-Verteilung ist glatter, da zu jedem Zeitpunkt nicht nur ein Wert, sondern so viele Werte eingehen, wie es Sites gibt. Auch für andere Dynamos stimmen diese beiden Kurven gut überein (nicht gezeigt). Die Dipol-Dispersionskurve hat allerdings gegenüber der VGP-Dispersionskuve meist ein etwas höheres und schmaleres Maximum.

Die graue Kurve von Abb. 4.14 unten zeigt die Verteilung der VGP's auf die Längenintervalle. Wäre der Wärmefluß homogen, müßte die Wahrscheinlichkeit für eine ausreichend lange Zeitreihe für alle Längen gleich groß sein, nämlich $P=100 \% / 360^{\circ}=0.278 \%$. Hier sind aber bei $0^{\circ}$ und $180^{\circ}$ deutlich Maxima sowie bei $90^{\circ}$ und $270^{\circ}$ Minima zu erkennen. Die Maxima (Minima) der VGP-Längenverteilung fallen mit den Maxima (Minima) des KMGWärmeflußmusters zusammen. Für die graue Kurve wurden alle VGP's benutzt, also auch die, die in hohen Breiten und somit geographisch nah aneinander liegen. Sinnvoller ist es, nur die transienten VGP's in die Analyse einzubeziehen, also solche, die hauptsächlich während Umpolungen und Exkursionen aufgezeichnet werden. Im Paläomagnetismus wählt man dazu z. B. alle VGP's, die mehr als $30^{\circ}$ von den Polen entfernt liegen (schwarze Kurve). Die blaue (orange) Kurve berücksichtigt schließlich nur VGP's, die mehr als $45^{\circ}\left(60^{\circ}\right)$ Poldistanz haben. Durch die Einschränkung auf transiente VGP's treten die Maxima und Minima der Kurve noch etwas besser hervor. In diesen Fällen ist die Wahrscheinlichkeit für eine VGP- 

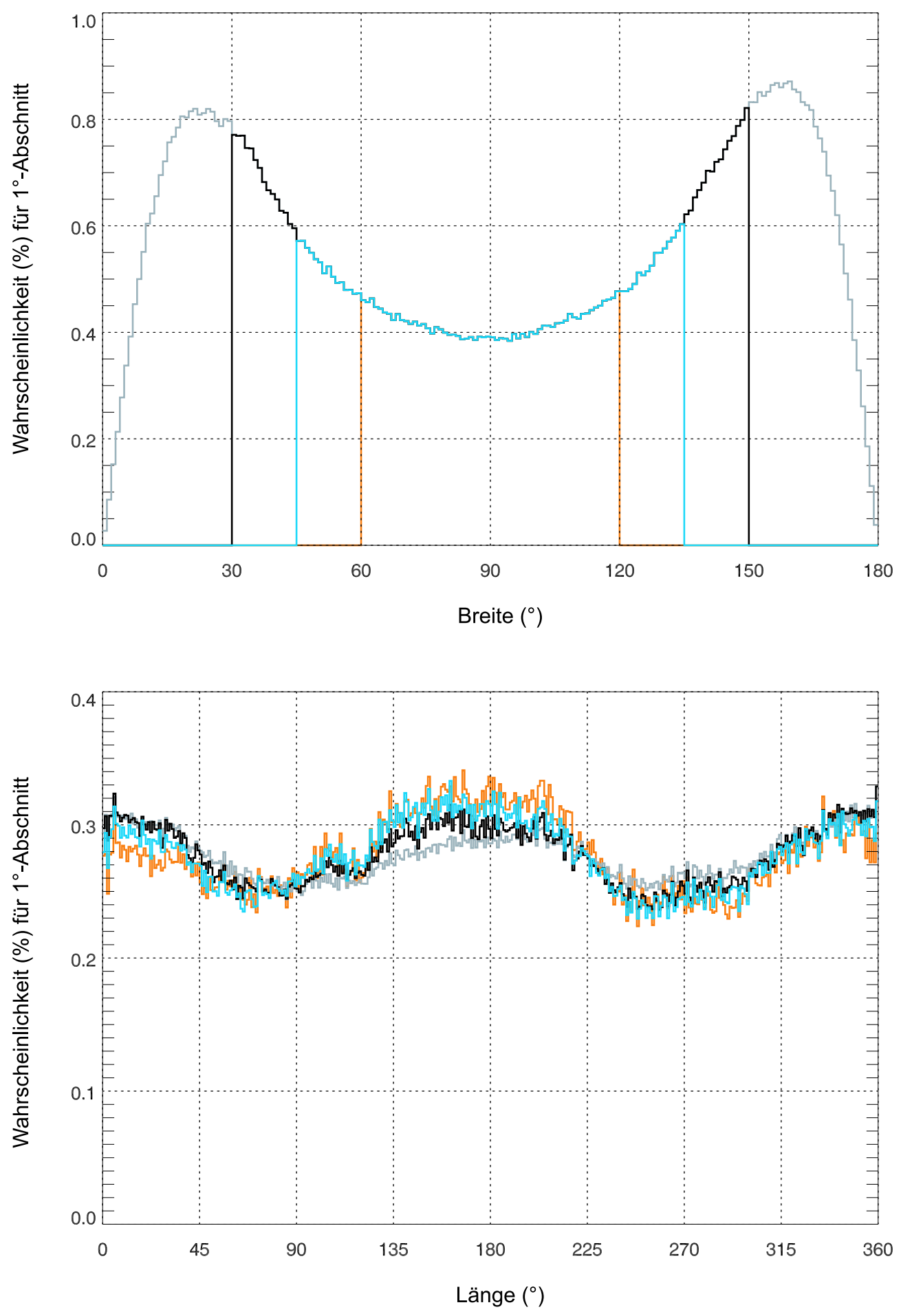

Abb. 4.14: VGP-Dispersionskurven: Prozentuale Wahrscheinlichkeit eines VGP's, in einen $1^{\circ}$-Abschnitt zu fallen. Oben: Verteilung der VGP's mit der Breite. Unten: Verteilung der VGP's mit der Länge. Für die untere Darstellung wurden VGP's berücksichtigt, die in einem Breitenfenster von $\pm 30^{\circ}$ (orange Kurve) um den Äquator liegen, bzw. $\pm 45^{\circ}$ (blau); $\pm 60^{\circ}$ (schwarz); $\pm 90^{\circ}$ (grau). 


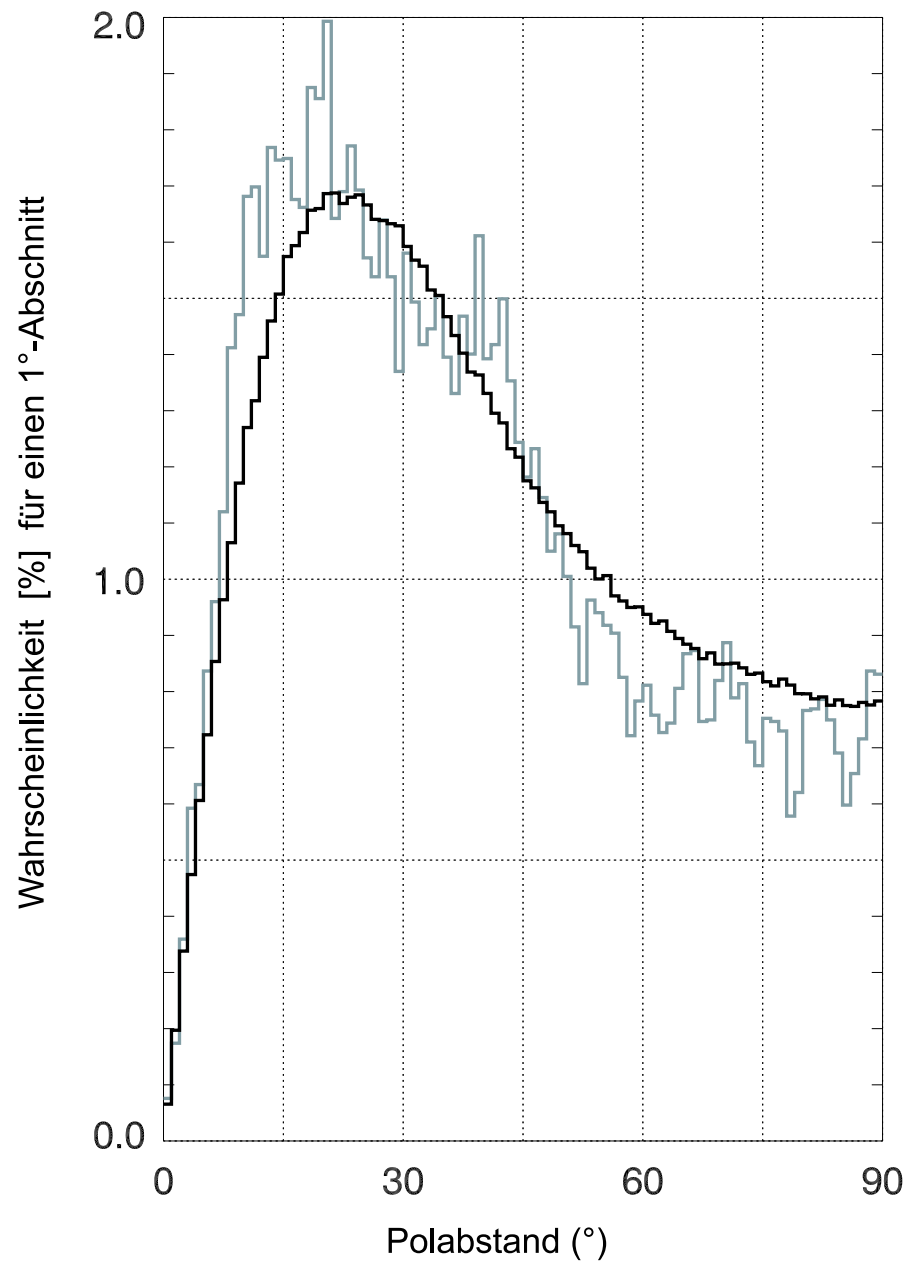

Abb. 4.15: Grau: Wahrscheinlichkeit für den Durchstoßpunkt der Dipolachse durch die Oberfläche, in $1^{\circ}$-Breitenabschnitte zu fallen. Schwarz: Wahrscheinlichkeit der VGP's, in $1^{\circ}$-Breitenabschnitte zu fallen. Für diese Darstellung wurde nicht zwischen magnetischem Nord- und Südpol unterschieden, daher reicht sie von $0^{\circ}$ (Pol) bis $90^{\circ}$ (Äquator). (Zeitreihe des Dynamos s. Abb.4.11) 


\begin{tabular}{|c|c|c|c|c|c|c|c|c|c|}
\hline & Modell & Ampl. & $\mathrm{Rm}$ & $\mathrm{Nu}$ & $\bar{E}_{\mathrm{mag}}$ & $\bar{E}_{\mathrm{mag}}^{\ell=1}$ & $\bar{B}_{\mathrm{KMG}}$ & $\bar{B}_{\text {dip }}$ & Neigung $\left[{ }^{\circ}\right]$ \\
\hline$a$ & homogen & 0.000 & 511 & 6.24 & 2960 & $1.5 \%$ & 0.34 & 0.052 & $\mathrm{r}$ \\
\hline$b$ & $l=1, m=0$ & -0.106 & 571 & 6.71 & 2910 & $3.0 \%$ & 0.24 & 0.039 & $\mathrm{r}$ \\
\hline c & $l=2, m=0$ & -0.106 & 473 & 6.26 & 3480 & $2.1 \%$ & 0.47 & 0.147 & $8.6(4.4)$ \\
\hline$d$ & $l=2, m=0$, inv. & 0.106 & 563 & 6.51 & 2190 & $1.2 \%$ & 0.25 & 0.025 & $\mathrm{r}$ \\
\hline $\mathrm{e}$ & $l=4, m=0$ & -0.106 & 534 & 6.33 & 2510 & $1.3 \%$ & 0.31 & 0.033 & $\mathrm{r}$ \\
\hline$f$ & $l=4, m=0$, inv. & 0.106 & 479 & 6.26 & 3950 & $2.0 \%$ & 0.46 & 0.130 & $12(8)$ \\
\hline g & $l=2, m=2$ & -0.106 & 520 & 6.40 & 3330 & $2.0 \%$ & 0.41 & 0.081 & $\mathrm{r}$ \\
\hline G & $l=2, m=2$ & -0.176 & 522 & 6.62 & 3500 & $2.0 \%$ & 0.39 & 0.084 & $\mathrm{r}$ \\
\hline $\mathrm{h}$ & tomographisch & 0.088 & 503 & 6.45 & 3270 & $2.0 \%$ & 0.37 & 0.074 & 19(12) \\
\hline $\mathrm{H}$ & tomographisch & 0.176 & 503 & 6.94 & 2080 & $1.1 \%$ & 0.28 & 0.026 & $\mathrm{r}$ \\
\hline
\end{tabular}

Tab. 4.2: Dynamodaten für unterschiedliche Wärmeflußmuster an der KMG bei den Parametern $\mathrm{E}=3 \times 10^{-4}, \mathrm{q}=3$ und $\mathrm{Ra}^{x}=9000$. Weitere Erklärungen s. Tab. 3.3

Lage über einem Maximum des Wärmeflusses etwa 30\% höher als über einem Minimum.

\subsection{Einfluß eines longitudinal variierenden KMG-Wärmeflusses auf die VGP-Verteilung}

Es soll nun der Einfluß des KMG-Wärmeflusses auf ein Modell mit den realistischeren Parametern $\mathrm{E}=3 \times 10^{-4}, \mathrm{q}=3$ und $\mathrm{Ra}^{x}=9000$ untersucht werden. Eine noch niedrigere Ekmanzahl und damit verbunden höhere Rayleighzahl konnte aus numerischen Gründen nicht gewählt werden. Bei der benutzten Auflösung von $\ell_{\max }=85$ bei $N=40$ radialen Gitterintervallen benötigt jedes Modell mehrere Wochen Rechenzeit, um eine Zeitreihe zu erzeugen, die ausreichend lang für eine statistische Auswertung ist.

Bei festgehaltenen dimensionslosen Parametern werden dem Dynamo die in Abb. 4.4 gezeigten Wärmeflußmuster aufgeprägt. Zunächst wird die mittlere Längenabhängigkeit der virtuellen Pole betrachtet; die Modelle b bis $f$ werden im nächsten Kapitel besprochen. Eine Variation des Wärmeflusses in $\phi$ ist im Modell der einzige mögliche Grund, der zu einer zeitgemittelten Häufung der VGP's in der Länge führen kann.

Als Anfangszustand für Modell $\mathrm{g}$ dient das Ergebnis des umkehrenden Dynamos aus den Abb. 3.10 (unten) bis 3.13 mit $\mathrm{E}=3 \times 10^{-4}, \mathrm{Ra}=31 \times \mathrm{Ra}_{\mathrm{krit}}, \mathrm{q}=3$. Die dimensionslosen Parameter sind dieselben, für die neue Simulation müssen lediglich die Randbedingungen an innerer und äußerer Grenze geändert werden. Als Anfangszustand für die anderen Modelle wird entweder ein Zustand des Modells g benutzt oder eines anderen schon modellierten Falls. Einige Kennwerte für diese Modelle sind in Tab. 4.2 zusammengestellt. Für die Modelle g und $\mathrm{h}$ wird auch der Einfluß der Amplitude des Wärmeflusses untersucht; die Modelle mit der jeweils höheren Amplitude werden mit den Großbuchstaben $\mathrm{G}$ und $\mathrm{H}$ gekennzeichnet. Bei der Berechnung der in der Tabelle gegebenen Mittelwerte blieben die ersten 1.0 Zeiteinheiten unberücksichtigt, um der Lösung Zeit zu geben, sich auf ihre neuen Randbedingungen einzustellen. In den Fällen $b, d$, und $h$ wurden die ersten 0.33 Zeiteinheiten ausgeschlossen. 


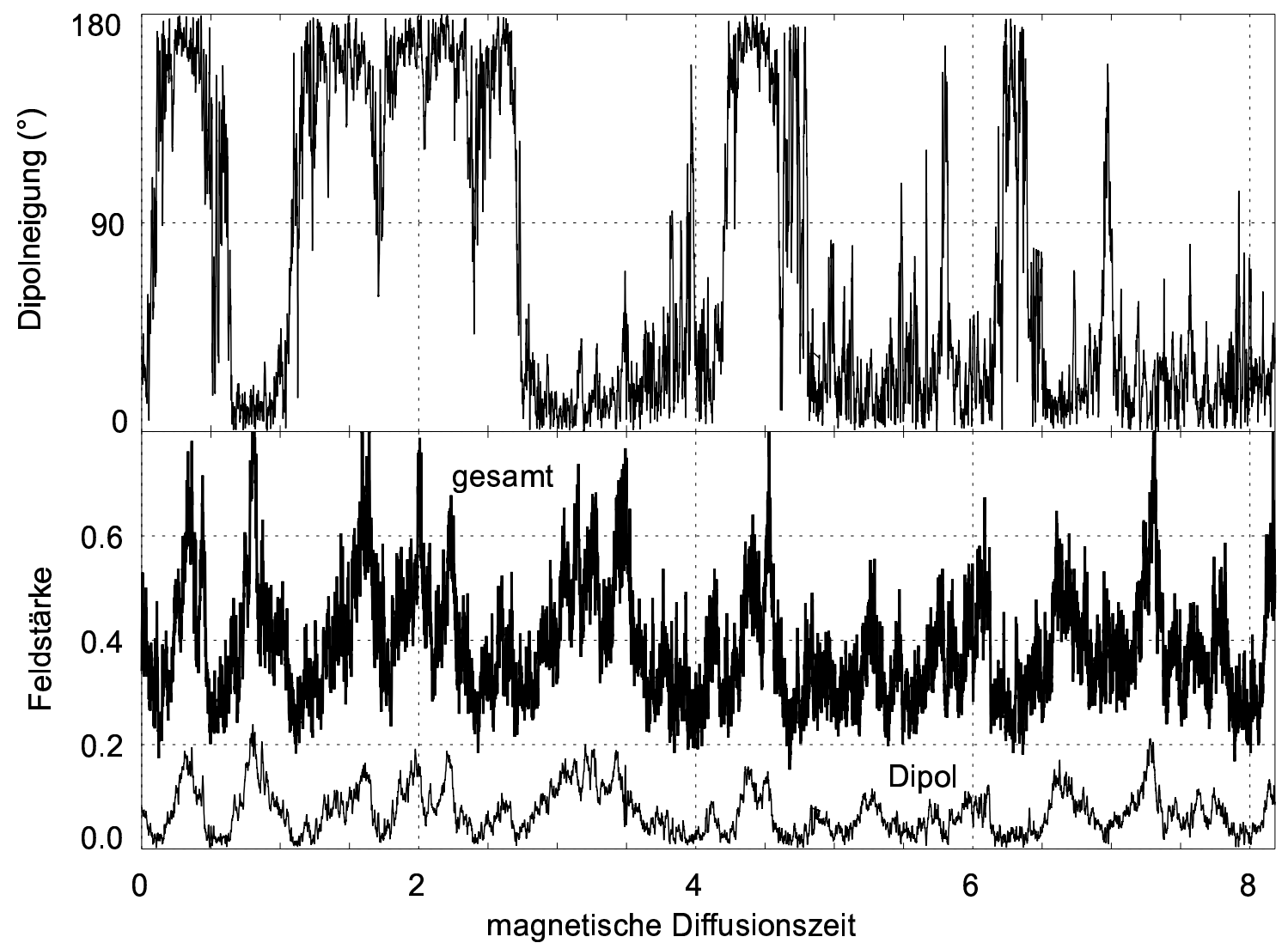

Abb. 4.16: Zeitreihe der Neigung der Dipolachse (oben) und der Feldstärke an der KMG (unten) des Modells g aus Tab.4.2 


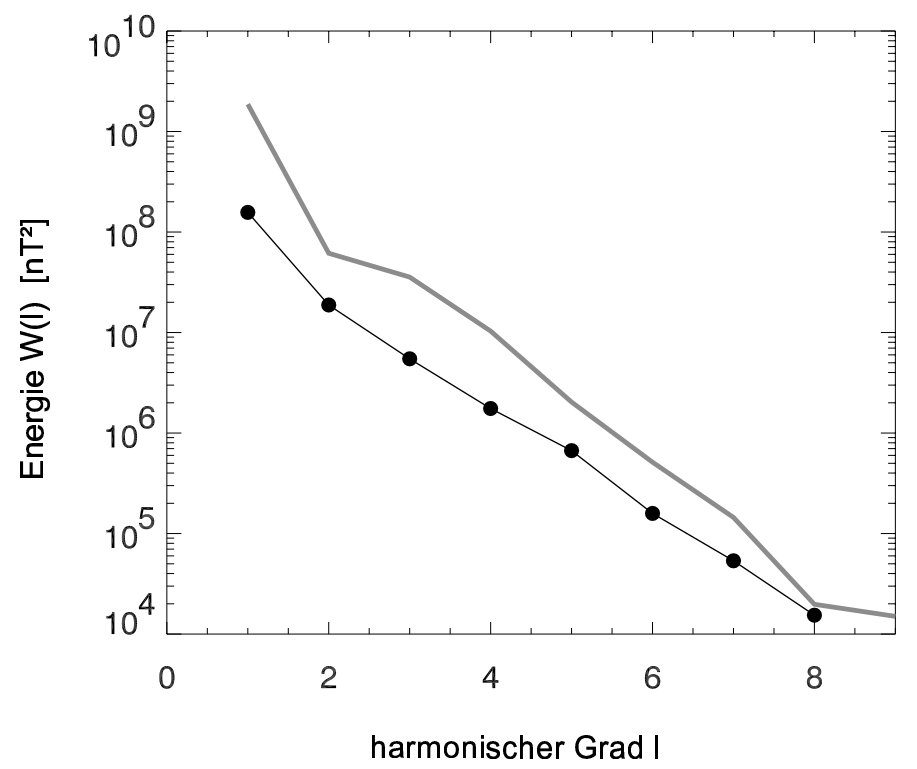

Abb. 4.17: Über 8 magnetische Diffusionszeiten gemitteltes Energiespektrum des Modelles g (Abb. 4.16) an der Oberfläche (schwarz, Punkte). Grau: Spektrum des Erdmagnetfeldes 1980 zum Vergleich.

In Abb.4.16 ist die Zeitreihe des Modells g mit Wärmeflußvariation in der $(\ell=2, m=2)$ Mode gezeigt. Die Simulation beinhaltet 8 Umkehrungen und einige Exkursionen. Das Dipolfeld macht an der KMG im Mittel ein Fünftel des Gesamtfeldes aus. Das Feld an der Oberfläche ist im zeitlichen Mittel dipol-dominiert, wie in Abb. 4.17 zu sehen ist (Berechnung des mittleren Spektrums s. Abschnitt 4.3.2). Die spektrale Energiedichte ist im Vergleich zum heutigen Erdmagnetfeld niedriger und fällt langsamer mit $\ell \mathrm{ab}$.

Zeichnet man für dieses Modell die VGP-Trajektorie, welche von einer Site am Äquator registriert wird, bemerkt man eine Häufung der VGP's bei Längen um $0^{\circ}$ und $180^{\circ}$ (Abb. 4.18). An diesen Längen hat der Wärmefluß aus dem Kern seine Maxima. Allerdings ist die Häufung der VGP's keineswegs so deutlich, wie von Laj et al. (1991) gesehen (Abb. 4.1).

Für das Modell g werden zunächst für die 200 Sites aus Abb. 4.13 die VGP's bestimmt und zeitgewichtet in 360 Breitenintervalle einsortiert (so wie es für Abb. 4.14 getan wurde). Die Untersuchung beschränkt sich auf transiente VGP's, also solche, die mindestens $30^{\circ}$ Poldistanz haben und somit in Zeiten von Umkehrungen und Exkursionen auftreten. Auch bei diesem Parametersatz gibt es ausgeprägte Maxima in der VGP-Verteilung an Orten hohen KMG-Wärmeflusses, was in Kap. 4.4.4 diskutiert wird. Vorher sollen einige Fragen bezüglich der Robustheit des Ergebnisses beantwortet werden:

- Wie stark hängt die VGP-Verteilung von der globalen Verteilung der Sites ab?

- Wie viele Sites braucht man, um die Maxima und Minima in der VGPDispersionskurve sehen zu können?

- Wie lang muß die Zeitreihe sein, um eine mittlere Häufung zu sehen? 


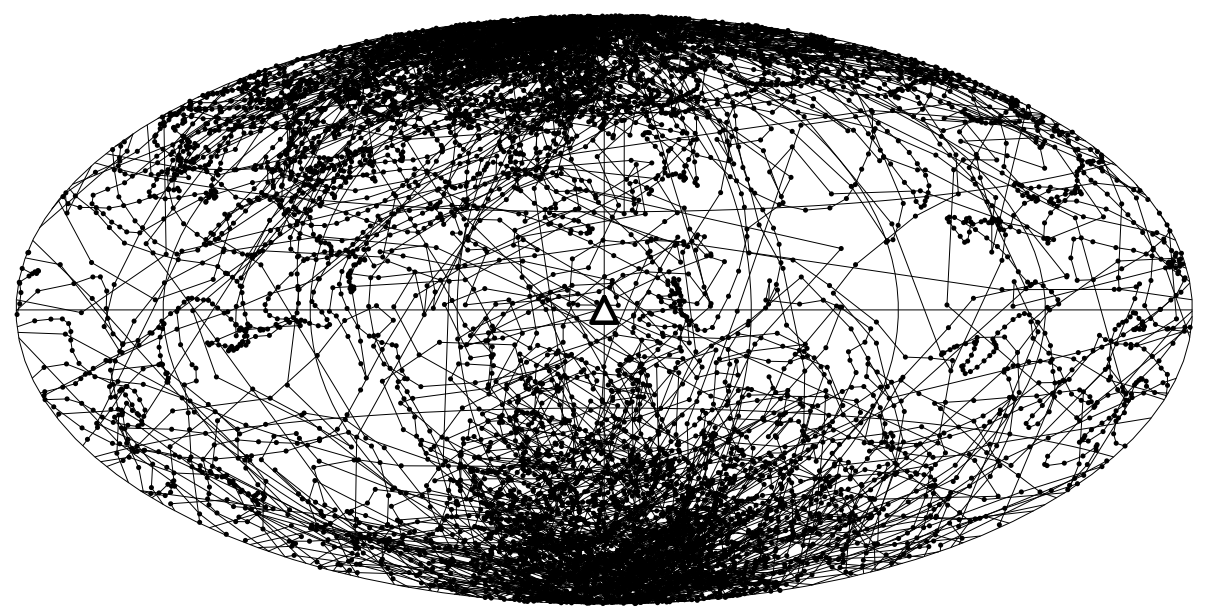

Abb. 4.18: VGP-Trajektorie für eine einzelne Site im Zentrum der Projektion (Pyramide bei $\theta=0^{\circ}, \phi=0^{\circ}$ ). Es wurden die Daten der ersten 4.3 Diffusionszeiten des Modells g aus Abb.4.16 verwendet.

- Sehen Sites aus verschiedenen Regionen ein unterschiedliches Verhalten?

\subsubsection{Verteilung und Anzahl der Sites}

Bis jetzt wurden die VGP's für Sites auf einem $10 \times 20$ Punkte großem, regelmäßigen Gitter bestimmt. Dabei sind die Sites in Äquatornähe recht äquidistant verteilt, zu den Polen hin liegen sie allerdings in der Länge immer dichter, je kleiner die Poldistanz ist (Abb.4.13). Der Einfluß der polaren Sites ist daher überproportional hoch. Besser ist eine Verteilung, bei der an den Polen genauso viele Sites pro Fläche vorhanden sind, wie in Äquatornähe. Dies wird näherungsweise durch eine zufällige Verteilung von Orten auf der Kugeloberfläche erreicht.

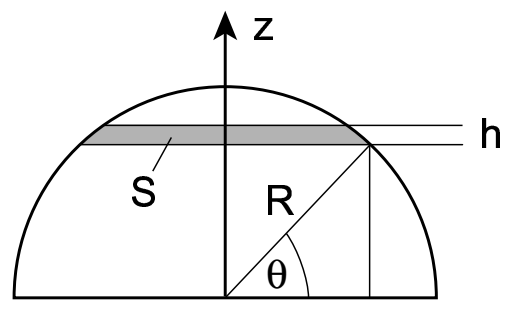

Abb. 4.19: Zur Erzeugung von gleichverteilten Punkten auf einer Kugeloberfläche vom Radius $R$. Der Flächeninhalt von $S$ (grau) hängt nur von $h$ ab, nicht von $z$.

Um diese Verteilung zu erzeugen, wird ausgenutzt, daß die Fläche $S$ eines Kugelmantels nur von der Dicke $h$ des Mantels abhängt, nicht aber von der Höhe $z$, in welcher er liegt (Abb. 4.19): $S=2 \pi R h$. Um eine konstante Anzahl an Punkten pro Fläche zu erhalten, genügt es, in jeden Kugelmantel $S$ bzw. in jeden Höhenabschnitt $\Delta z=h$ gleich viele Punkte zu positionieren. Dafür werden gleichverteilte Zufallszahlen im Intervall $z=-R \cdots+R$ erzeugt, welche über die Beziehung $\sin \theta=z / R$ in Breiten $\theta$ umgerechnet werden. Die Längen wer- 


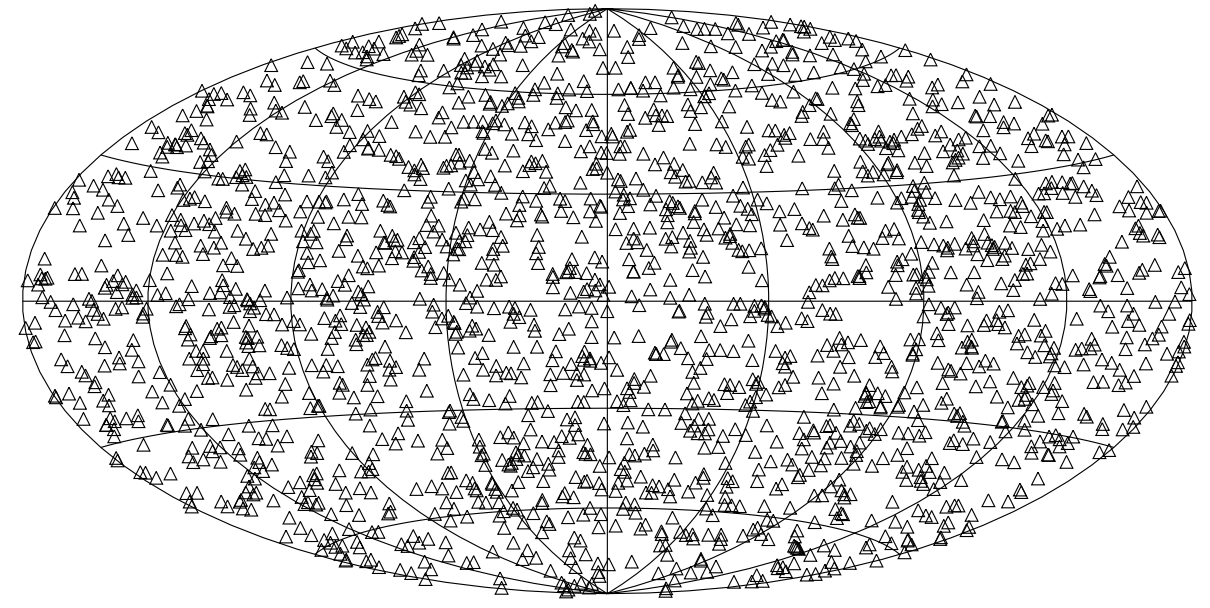

Abb. 4.20: Hammer-Projektion mit 2000 zufällig verteilten Sites (Pyramiden).

den zufällig und gleichverteilt gewählt. Statistisch erhält man pro Fläche die gleiche Anzahl an Punkten. Ein Beispiel der Verteilung von 2000 Sites gibt Abb.4.20

Der Einfluß der Anzahl und Verteilung der Sites auf die VGP-Dispersionskurve ist in Abb. 4.21 zu sehen. Für Abbildung a) wurde eine sehr dichte Abdeckung der Erdoberfläche mit 2000 Sites gewählt. Die schwarze Kurve zeigt die VGP-Verteilung für ein regelmäßiges Gitter, welches aus 32 Punkten in $\theta$ - und 63 Punkten in $\phi$-Richtung besteht. Die blaue Kurve erhält man für die 2000 zufälligen Sites aus Abb. 4.20, Die rote Kurve gilt für eine andere zufällige Verteilung von 2000 Sites.

In den beiden Fällen der 2000 zufälligen Sites liegen die Dispersionskurven dicht beieinander, woraus geschlossen werden kann, daß die VGP-Verteilung nicht von den genauen Koordinaten der Sites abhängt, wenn nur eine ausreichend hohe Anzahl an Sites zufällig verteilt liegt. Die Kurve für das regelmäßige Gitter unterscheidet sich allerdings von den anderen Kurven; bei ihr sind die Extrema etwas ausgeprägter.

Diese Beobachtungen lassen sich auch noch machen, wenn man die Anzahl der Sites um den Faktor 10 erniedrigt, was in Abbildung b) gezeigt ist. Im Falle einer weiteren Reduzierung der Anzahl der Sites auf 20 in Abb. c) sieht die Kurve etwas verrauscht aus. Ein Einfluß der Gittergeometrie ist nicht mehr zu erkennen, wohl aber sind die Extrema noch gut zu sehen. Diese lassen sich in Abb. d) (2 Sites) nur noch vermuten. Auch durch die Vergrößerung der Breitenintervalle, in die die VGP's einsortiert werden, von $1^{\circ}$ auf $10^{\circ}$ läßt sich bei nur 2 Sites nicht wesentlich mehr erkennen.

Um robuste Aussagen über die Lage und Höhe der Extrema der VGP-Dispersionskurven zu machen, wird für die folgenden Untersuchungen eine Anzahl von mindestens 200 Sites in zufälliger Verteilung benutzt. Selbst bei 20 Sites sind aber die Häufungseffekte noch deutlich zu erkennen.

\subsubsection{Länge der Zeitreihe}

Um herauszufinden, wie lang die Zeitreihe eines Dynamos mit heterogenem Wärmefluß sein muß, damit eine mittlere Häufung der VGP's in der Dispersionskurve erkennbar ist, werden diese Kurven für unterschiedlich lange Zeitintervalle erzeugt. Das Ergebnis ist in Abb. 4.22 


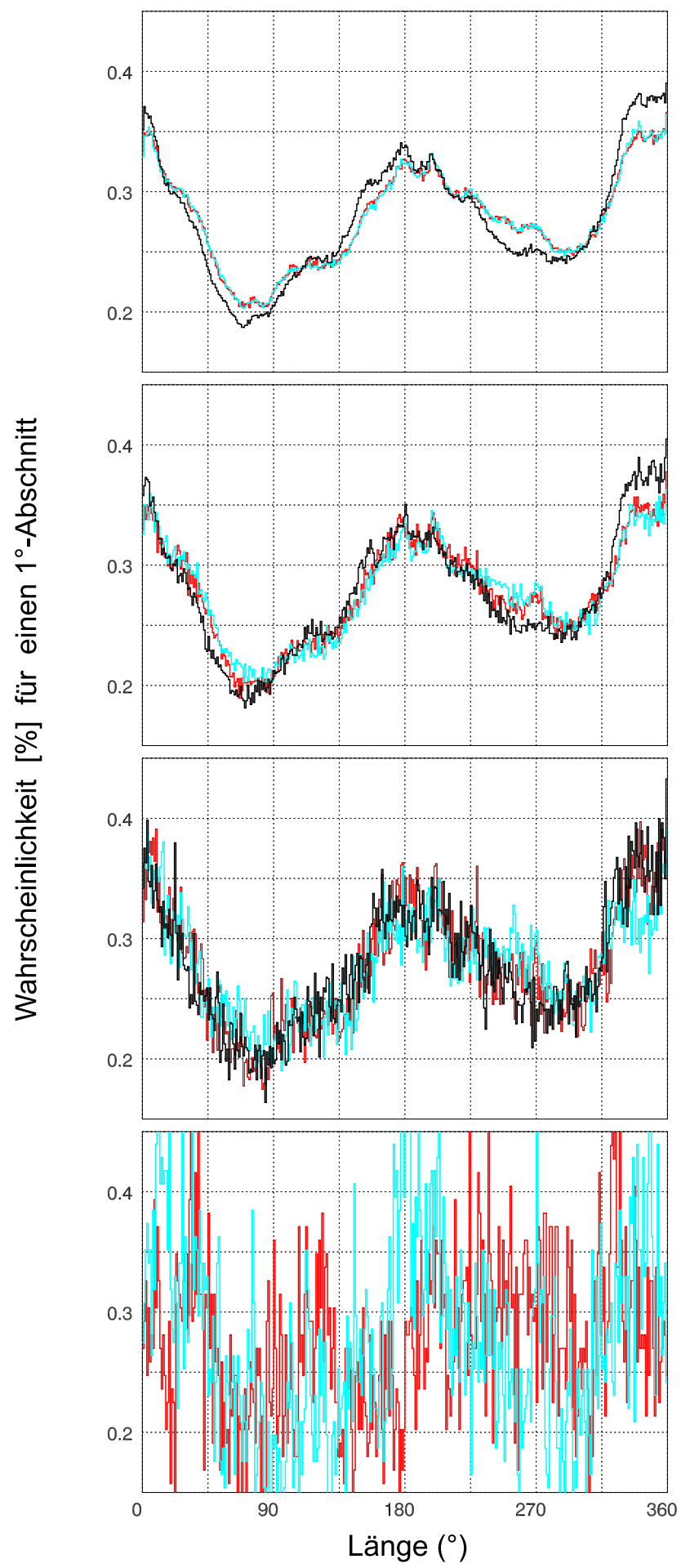

a)

b)

c)

d)

Abb. 4.21: VGP-Dichte als Funktion der Länge für verschiedene Sites, die auf einem regelmäßigen Gitter liegen (schwarz) und für zwei zufällige Verteilungen (rot und blau). a) 2000 Sites (bzw. beim regelmäßigen Gitter $32 \times 63=2016$ ); b) 200 Sites (bzw. $20 \times 10$ ); c) 20 Sites (bzw. $3 \times 7$ ); d) 2 Sites in 2 zufälligen Verteilungen. Die VGP's wurden für die ersten 4.7 magnetischen Diffusionszeiten des Dynamos aus Abb. 4.16 bestimmt. 


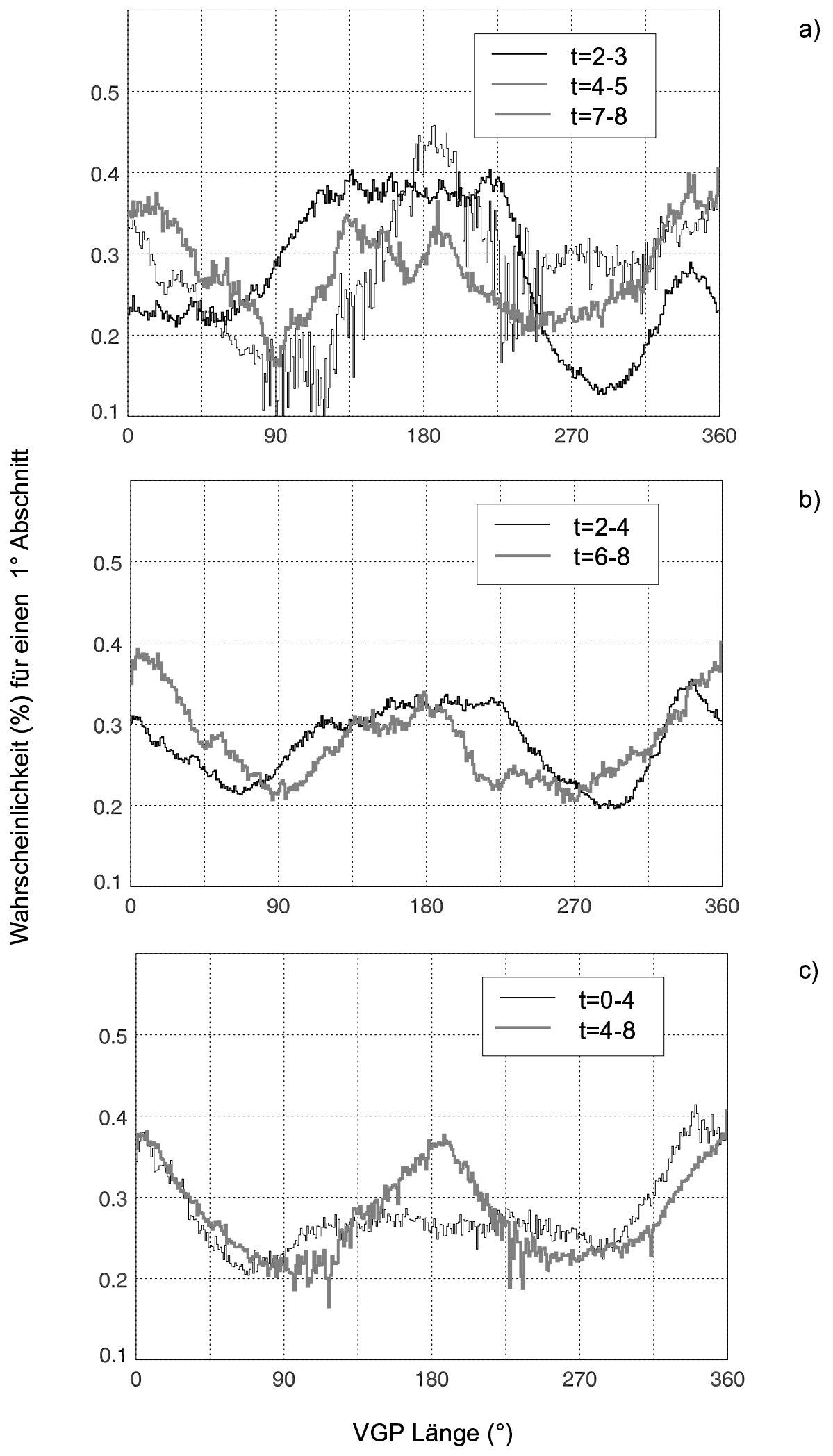

Abb. 4.22: VGP-Dichte von Modell g als Funktion der geographischen Länge für $N \geq 200$ zufällig gewählte Sites. Länge $\Delta t$ der Zeitreihe in magnetischen Diffusionszeiten: a) $\Delta t=1$, b) $\Delta t=2$, c) $\Delta t=4$. 
gezeigt.

In Abb. a) sind drei Kurven für verschiedene Zeitintervalle der Länge $\Delta t=1.0$ gezeigt. Obwohl alle Kurven Maxima bei etwa $0^{\circ}$ und $180^{\circ}$ und Minima dazwischen haben, ist die Höhe und Breite der Extrema sehr unterschiedlich. Verdoppelt man die Länge des Zeitintervalls (b), erhält man mehr Ähnlichkeit der sich aus verschiedenen Zeitintervallen ergebenden Kurven, dieser Trend setzt sich in c) für $\Delta t=4.0$ fort. Zu schwarzen Kurve in c) ist zu bemerken, daß das $180^{\circ}$-Maximum unerwartet schwach ausgeprägt ist. Der Grund ist wahrscheinlich, daß diese Kurve auch den Anfangsbereich der Zeitreihe einschließt, zu dem sich das Magnetfeld noch nicht auf die neu aufgeprägte Wärmeflußvariation eingestellt hat.

In der grauen Kurve für $t=4$ bis $t=8$ sieht man sehr gut die $180^{\circ}$-Periodizität, welche aufgrund der zweifachen Längensymmetrie des Wärmeflußmusters zu erwarten ist. Auch sind die Höhen der Maxima und Minima jeweils ungefähr gleich, was dafür spricht, daß diese Kurve ein repräsentatives Mittel darstellt. Die Extrema liegen bei etwa 0.20 und 0.37, was auch schon in b) erkennbar ist, nicht aber in a). Eine Mittelung über eine magnetische Diffusionszeit ist nicht ausreichend, ab $\Delta t=2$ kann man aber den prinzipiellen Einfluß des Wärmeflusses sehen.

\subsubsection{Sites über hohem und niedrigem Wärmefluß}

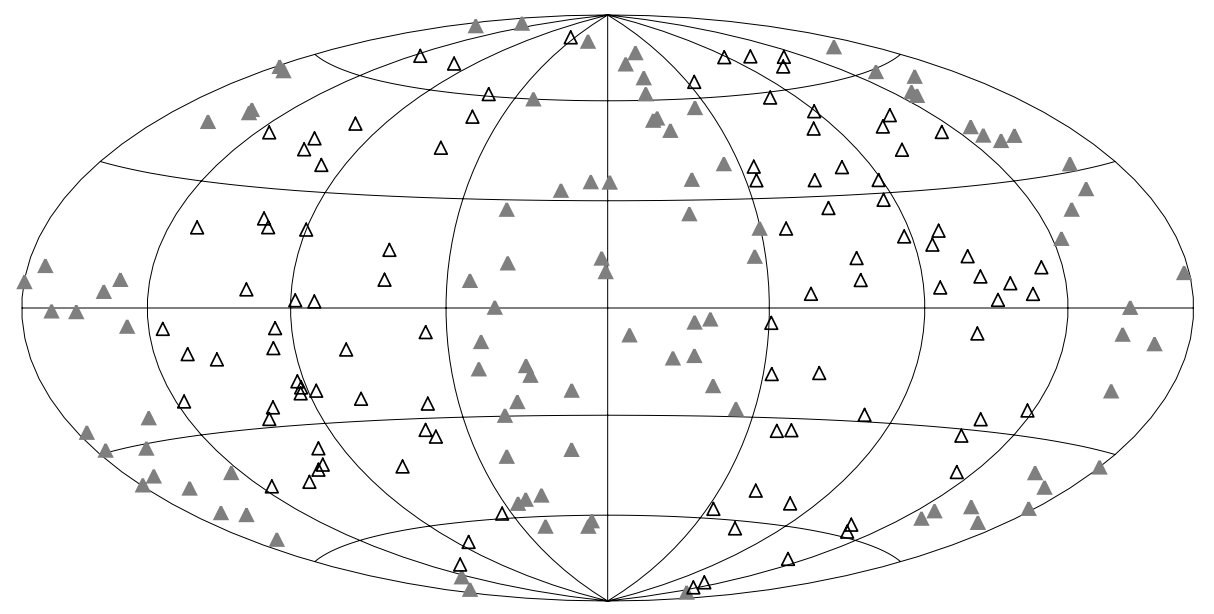

Abb. 4.23: Die grauen (schwarzen) Pyramiden stehen für 100 zufällige Sites über Gebieten mit hohem (niedrigem) KMG-Wärmefluß. $\phi=\theta=0$ liegt im Zentrum der Projektion.

Bei einer global gleichmäßigen Verteilung der Sites liegen die virtuellen Pole gehäuft über Regionen mit hohem KMG-Wärmefluß. Es soll noch überprüft werden, ob im zeitlichen Mittel die Sites in unterschiedlichen Regionen das gleiche sehen, oder ob man unterschiedliche VGP-Verteilungen erhält, je nachdem, ob die Sites über Regionen mit hohem oder niedrigem Wärmefluß liegen.

Abb. 4.23 zeigt die für diesen Test verwendete Verteilung der Sites. Es sind je 100 Sites über den Regionen mit hohem bzw. mit niedrigen Wärmefluß. Abb. 4.24 zeigt die zugehö- 


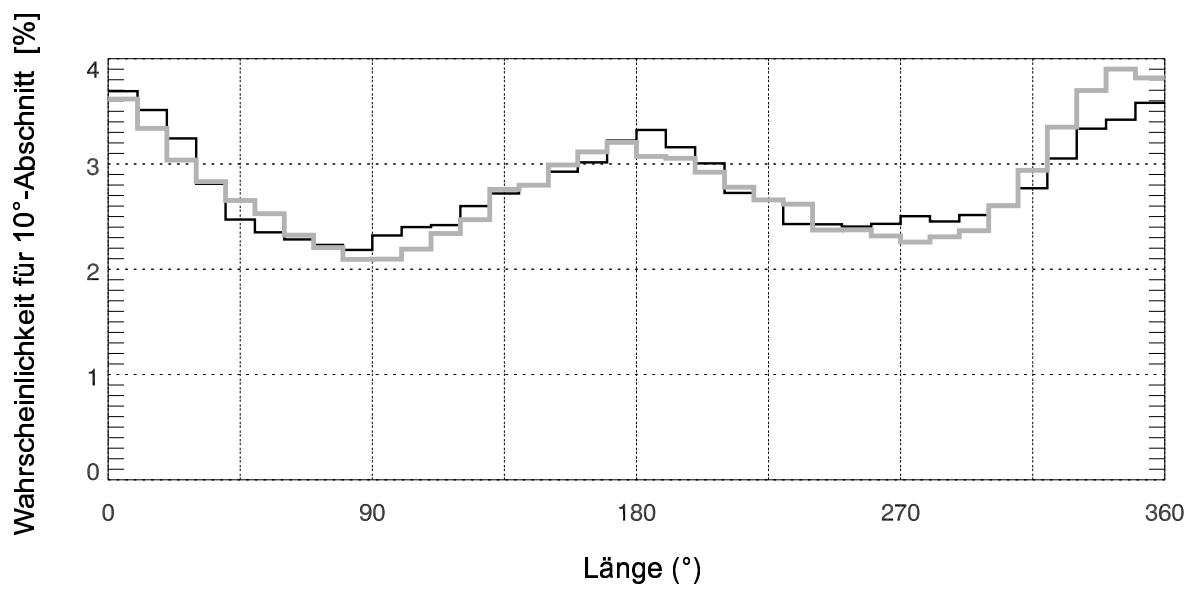

Abb. 4.24: VGP-Dispersionskurve für Modell g für die Sites aus Abb. 4.23 Graue (schwarze) Kurve: Sites liegen in den Gebieten mit hohem (niedrigem) Wärmefluß. Mittel über 8.2 Diffusionszeiten.

rigen Dispersionskurven. Die Sites über Regionen hohen Wärmeflusses sehen die gleiche Dispersionskurve wie die Sites über Regionen niedrigen Wärmeflusses.

Jetzt wird die Region, in die die Sites gelegt werden, noch weiter verkleinert, und zwar auf den Bereich hohen Wärmeflusses zwischen $-45^{\circ}<\phi<+45^{\circ}, 90^{\circ}<\theta<0^{\circ}$ bzw. auf den Bereich niedrigen Wärmeflusses, der um $90^{\circ}$ in der Länge verschoben liegt. Auch diese weitere Einschränkung verändert die Dispersionskurve nicht merklich (nicht gezeigt).

Sofern man über einen ausreichend langen Zeitraum mittelt, erhält man unabhängig von der Wahl der Sites die gleiche Dispersionskurve. Für kurze Zeitintervalle wird dies im allgemeinen nicht gelten, da dann lokale Eigenschaften des Oberflächenfeldes ausschlaggebend werden.

\subsubsection{Mittlere Verteilung der virtuellen Pole}

Abb. 4.25 zeigt die Verteilung der virtuellen Pole, die man erhält, wenn man über 8.5 Diffusionszeiten mittelt. Die Einordnung der VGP's erfolgt ab jetzt in $10^{\circ}$ große Abschnitte. Im Paläomagnetismus werden VGP's oft in noch größere Intervalle von z. B. $30^{\circ}$ eingeteilt. Die Wahrscheinlichkeit für einen VGP, in das Intervall an der Stelle des höchsten KMGWärmeflusses zu fallen, ist in diesem Modell etwa 60\% höher, als die Wahrscheinlichkeit, in das Intervall über dem Wärmeflußminimum zu fallen.

Im unteren Bild von Abb. 4.25 ist die VGP-Verteilung zusätzlich nach der Breite aufgeschlüsselt. Dazu wird über die Oberfläche ein $10^{\circ} \times 10^{\circ}$-Gitter gelegt, in welches die VGP's zeitgewichtet einsortiert werden. Von den so erhaltenen $36 \times 12$ Werten wird ein Konturplot gemacht, welcher so normiert wird, daß sein Maximalwert 1.0 ist. Die Abbildung beschreibt also die relative Häufigkeit von VGP's in einem Fenster gegebener Länge $\times$ Breite.

Obwohl der Dynamo einige Male umkehrt, befinden sich die meisten transienten VGP's in Breiten $\theta>40^{\circ}$. In dieser Eigenschaft ähnelt die VGP-Verteilung der der MatuyamaBrunhes-Umkehr (Abb. 4.3). In Äquatornähe $\left(-30^{\circ}<\theta<+30^{\circ}\right)$ ist die VGP-Dichte sehr 


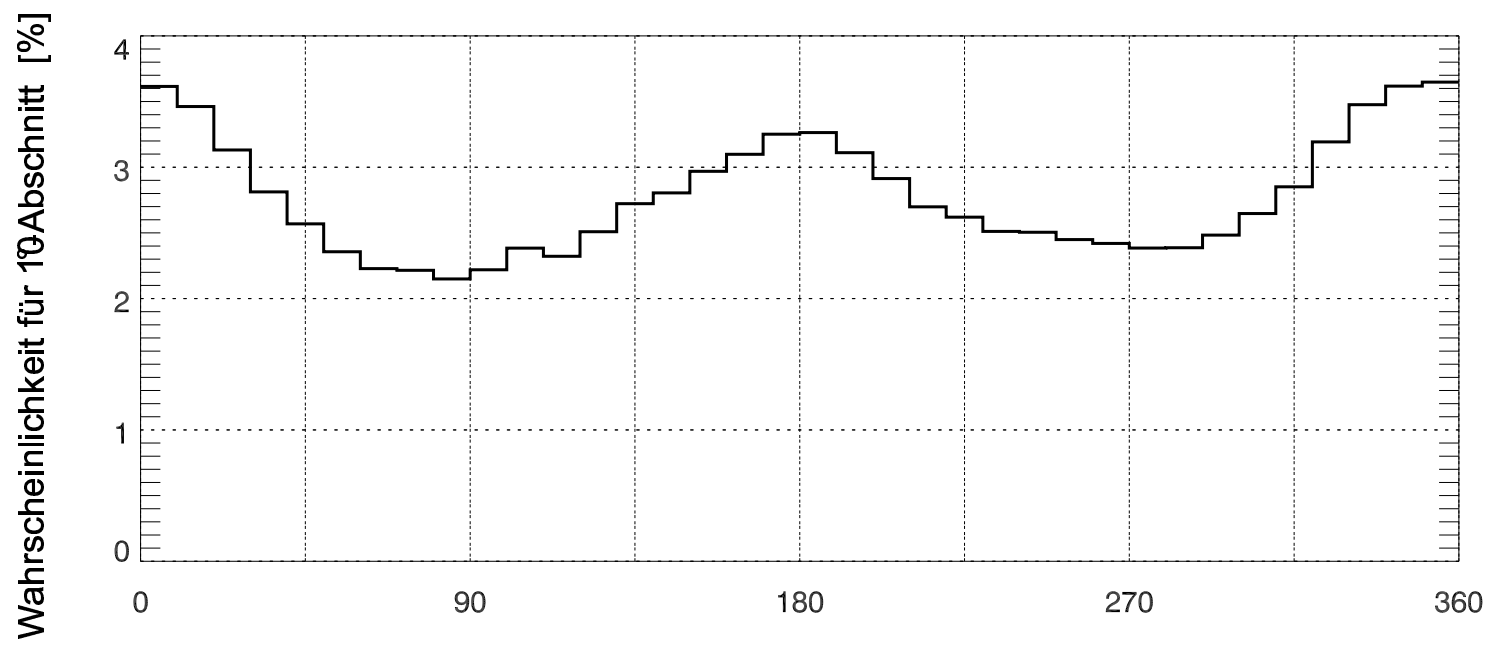

Länge $\left({ }^{\circ}\right)$

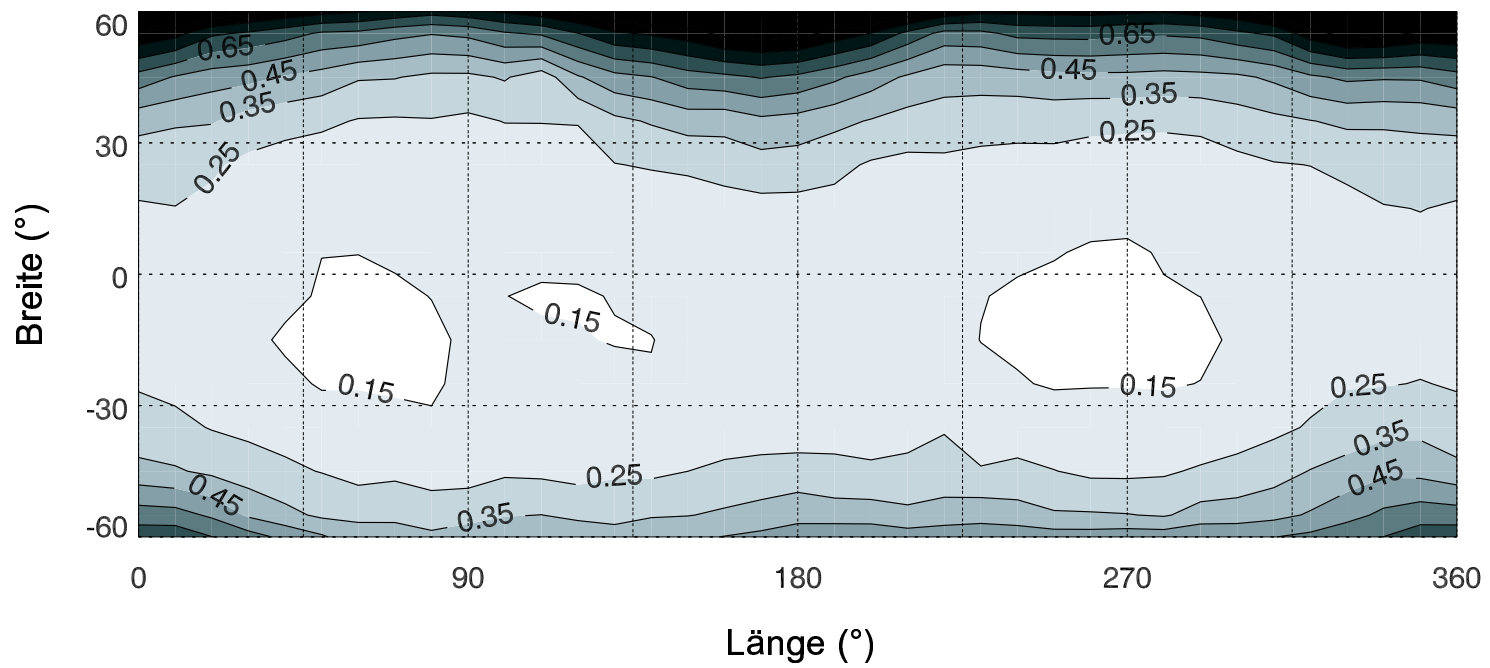

Abb. 4.25: VGP-Dichte des Modells g für 200 zufällige Sites über 8.5 Diffusionszeiten gemittelt. Oben: Wahrscheinlichkeit für einen VGP, in ein $10^{\circ}$ Längenintervall zu fallen. Unten: Anzahl an VGP's pro $10^{\circ} \times 10^{\circ}$ Intervall, normiert, so daß der Maximalwert im Konturplot 1.0 ist. Die Wärmeflußmaxima an der KMG des Modells liegen bei $0^{\circ}$ und $180^{\circ}$. 


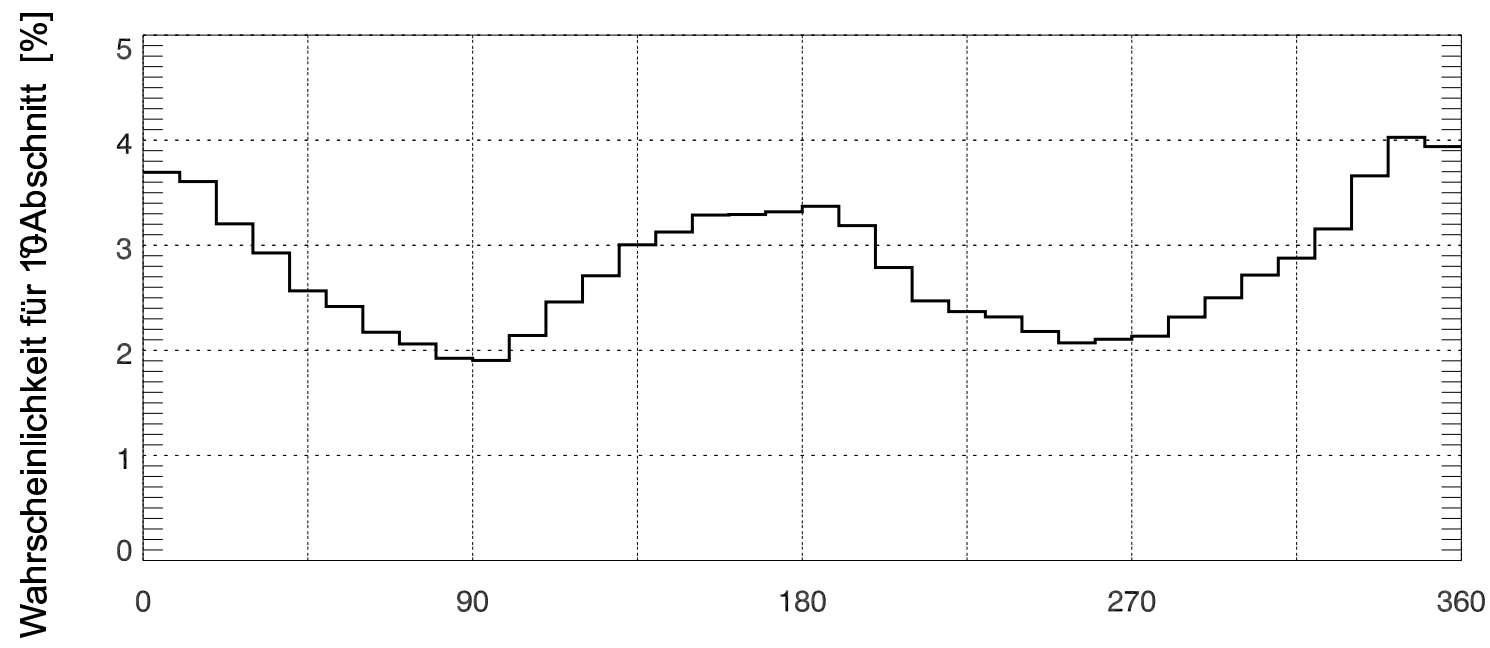

Länge $\left({ }^{\circ}\right)$

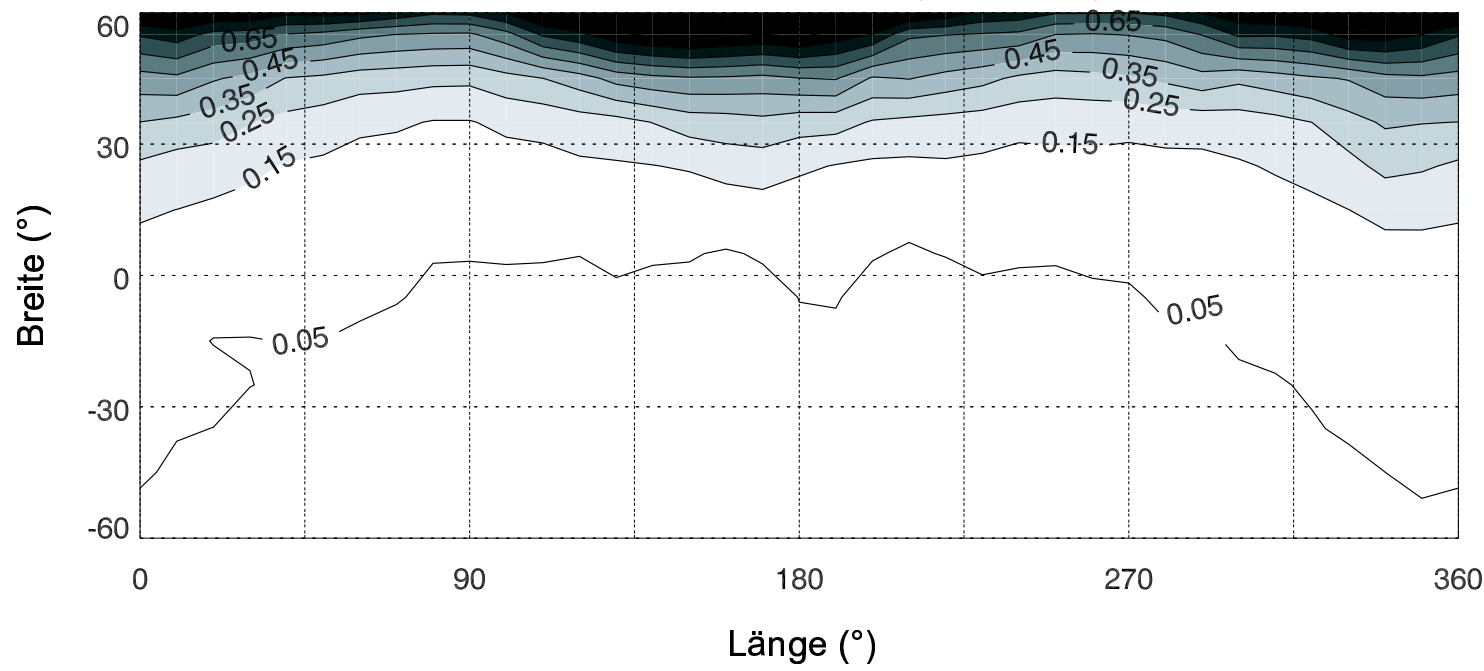

Abb. 4.26: Stabile Periode von Modell g ohne Umkehrungen zwischen $t=6.5$ und $t=8.5$. Darstellung wie in Abb. 4.25Darstellung wie in Abb. 4.33

gering. Allerdings ist auch am Äquator die VGP-Dichte über den Wärmeflußmaxima höher als über den Minima. Da sich der Dynamo während des untersuchten Zeitraums öfter in normaler als in inverser Polarität befindet, liegen mehr VGP's in der Nordhemisphäre.

Beschränkt man die Analyse auf einem Zeitraum stabiler Polarität, erhält man Abb. 4.26 Bis auf Exkursionen ist der Dynamo während des betrachteten Zeitintervalls immer in der gleichen Polarität, was zur Folge hat, daß der Großteil der virtuellen Pole in der Nordhemisphäre liegt. Der relative Dipolbeitrag an der Oberfläche beträgt im Mittel etwa 0.9, wenn man von den Zeiten der Exkursionen absieht. Auch ohne Umpolungen ergibt sich die bekannte VGP-Dispersionskurve; zumindest in der Längenverteilung der VGP's kann man keinen wesentlichen Unterschiede zum Fall mit Umkehrungen Abb. 4.25 erkennen. Die etwas 

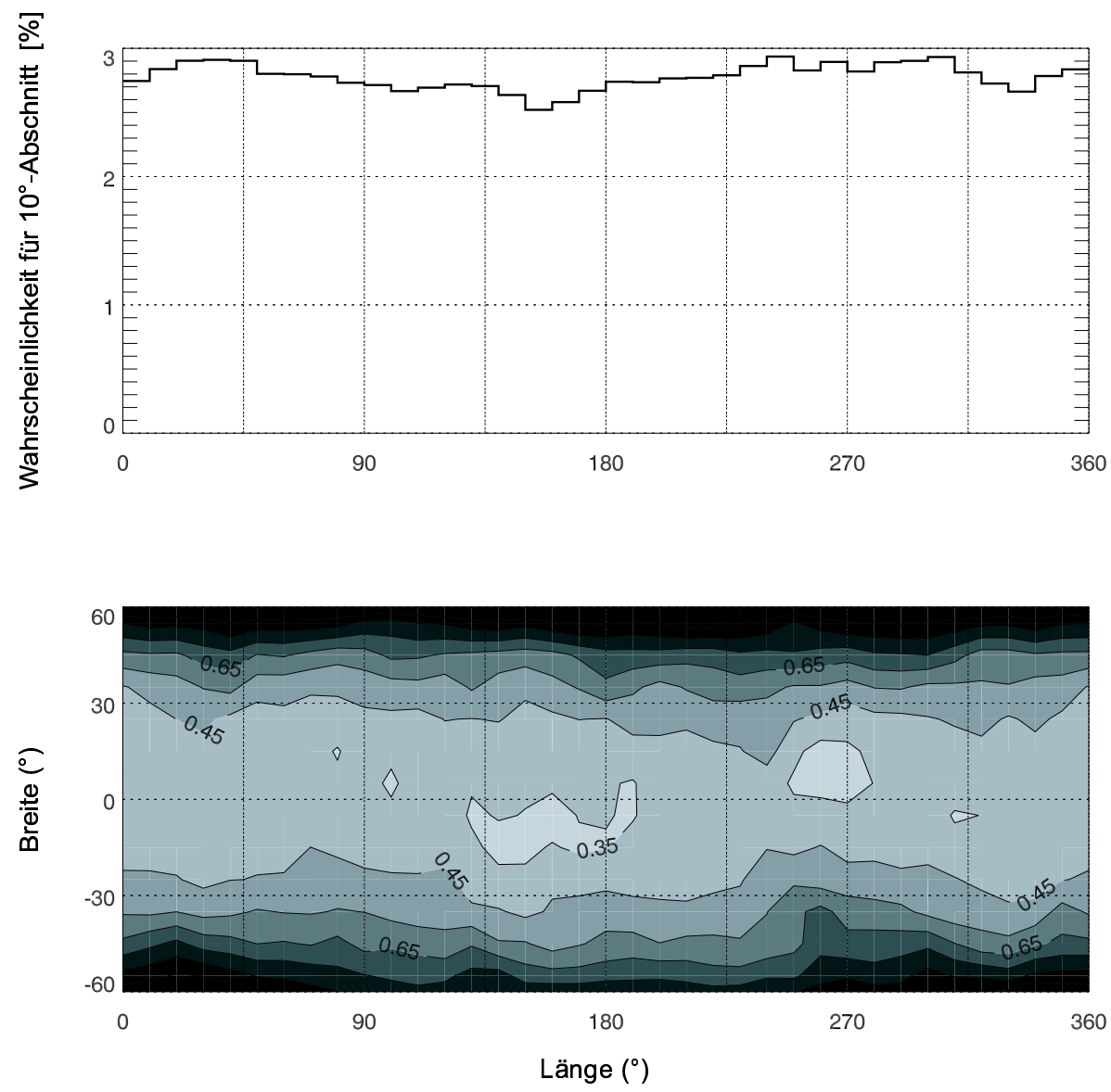

Abb. 4.27: Darstellung wie in Abb.4.25 hier für die 3.6 Diffusionszeiten lange Zeitreihe des Modells a mit homogenem Wärmefluß.

größere Differenz zwischen Maximum und Minimum der Kurve kann auf das kürzere Mittelungsintervall von zwei Diffusionszeiten zurückgeführt werden.

Abb. 4.27 zeigt zum Vergleich die VGP-Verteilung für einen Dynamo mit homogenem KMG-Wärmefluß (Modell a). Es gibt nur eine sehr geringfügige Variation der VGP-Dichte mit der Länge, die darauf zurückzuführen ist, daß die Zeitreihe nur 3.6 Diffusionszeiten lang ist. Nach ausreichend langer Zeit muß die Längenabhängigkeit natürlich ganz verschwinden.

\subsubsection{Verschiedene Amplituden der Modulation}

Um einen möglichen Einfluß der Stärke der Wärmeflußvariation auf die VGP-Streuung festzustellen, wird das Modell $\mathrm{g}$ aus Tab. 4.2 mit dem Modell $\mathrm{G}$ verglichen, bei dem die Amplitude der Variation $66 \%$ höher ist. In Abb. 4.28 sind die beiden VGP-Dispersionskurven zusammen gezeigt. Möglicherweise zeigt die Dispersionskurve bei der höheren Amplitude etwas ausgeprägtere Maxima und Minima, dies ist jedoch nach der in diesem Fall nur 3 Diffusionszeiten umfassenden Zeitreihe noch nicht klar sichtbar. Die Amplitude der Dispersionskurve zwischen Minimum und Maximum ist hier 1.6 mal so groß wie bei schwächeren Modulation. 


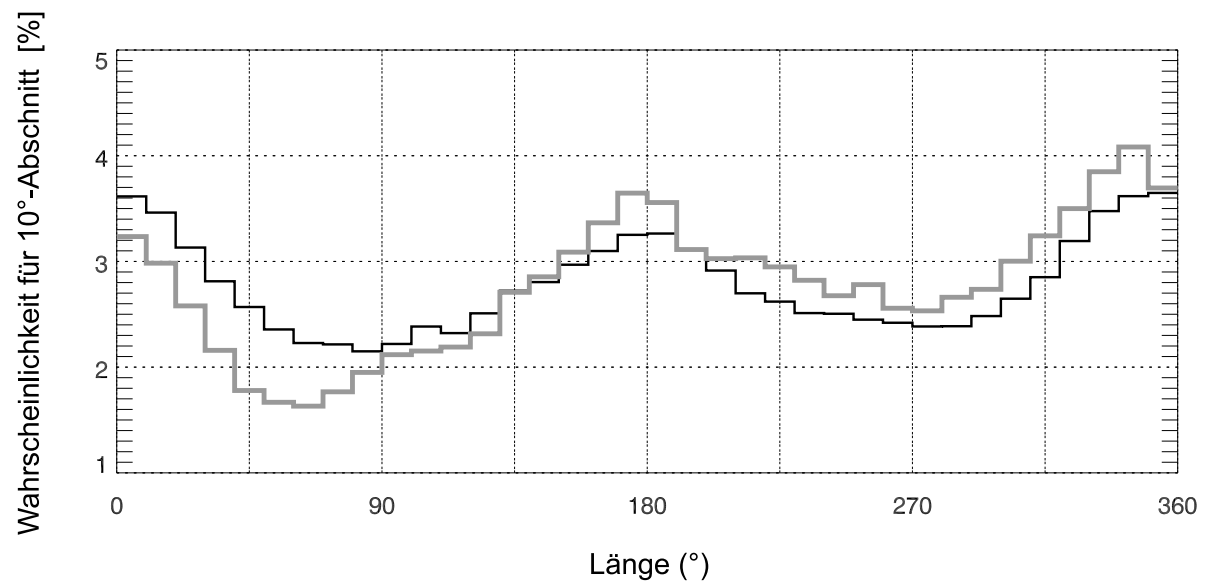

Abb. 4.28: VGP-Dispersion für 200 zufällige Sites bei zwei verschieden starken Amplituden der Modulation. Schwarze Linie: Modell g mit $f_{0}(2,2)=0.106$, Mittel über 8 magnetische Diffusionszeiten; graue Linie: Modell G mit $f_{0}(2,2)=0.176$, Mittel über 2 Diffusionszeiten.

\subsubsection{Tomographische Modulation}

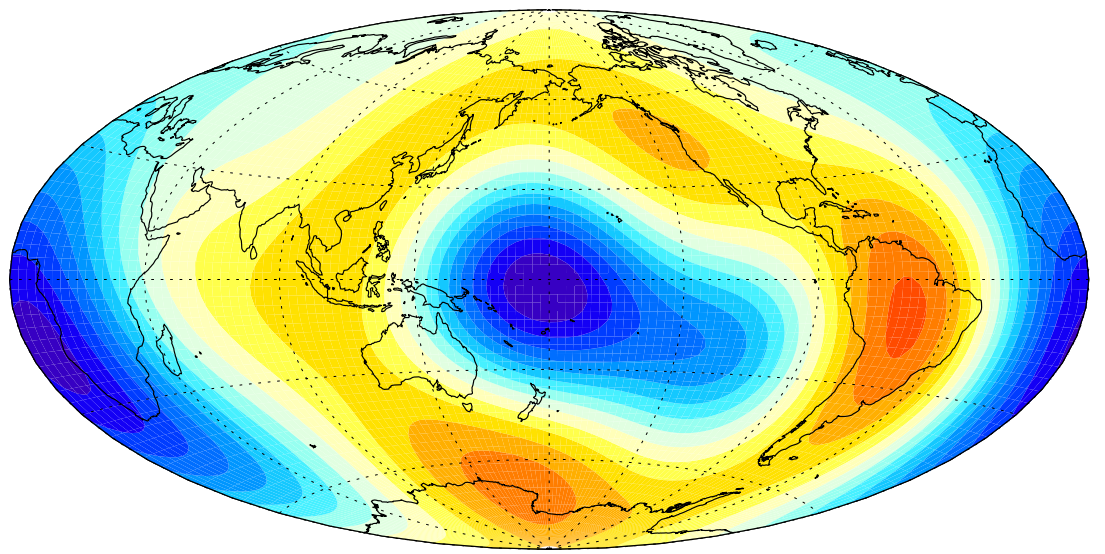

Abb. 4.29: Wärmefluß an der KMG für die tomographischen Modelle $\mathrm{h}$ und $\mathrm{H}$. Überadiabatischer Wärmefluß in Rottönen, unteradiabatischer in Blautönen. Die geographische Länge von $0^{\circ}$ (Greenwich-Meridian) befindet sich am linken Rand, die Länge $180^{\circ}$ im Zentrum der Projektion.

Zum Abschluß der Untersuchungen über mittlere VGP-Verteilungen betrachten wir die Modelle $\mathrm{h}$ und $\mathrm{H}$, in denen dem Dynamo ein aus der seismischen Tomographie abgeleitetes Wärmeflußmuster aufgeprägt ist (Abb. 4.29). $\mathrm{Zu}$ dem tomographischen Wärmeflußmuster liefert die $(\ell=2, m=2)$-Mode den größten Beitrag. Es ist also zu erwarten, daß das Ergebnis ähnlich aussieht, wie bei Modell g. Die Amplitude dieser Mode wurde in $\mathrm{H} \mathrm{zu}$ $f_{0}(2,2)=0.176$ gewählt und in h halb so groß zu $f_{0}(2,2)=0.088$. Die Amplituden der anderen Moden wurden relativ zum Wert der Mode $(\ell=2, m=2)$ skaliert. In Abb. 4.30 sind 


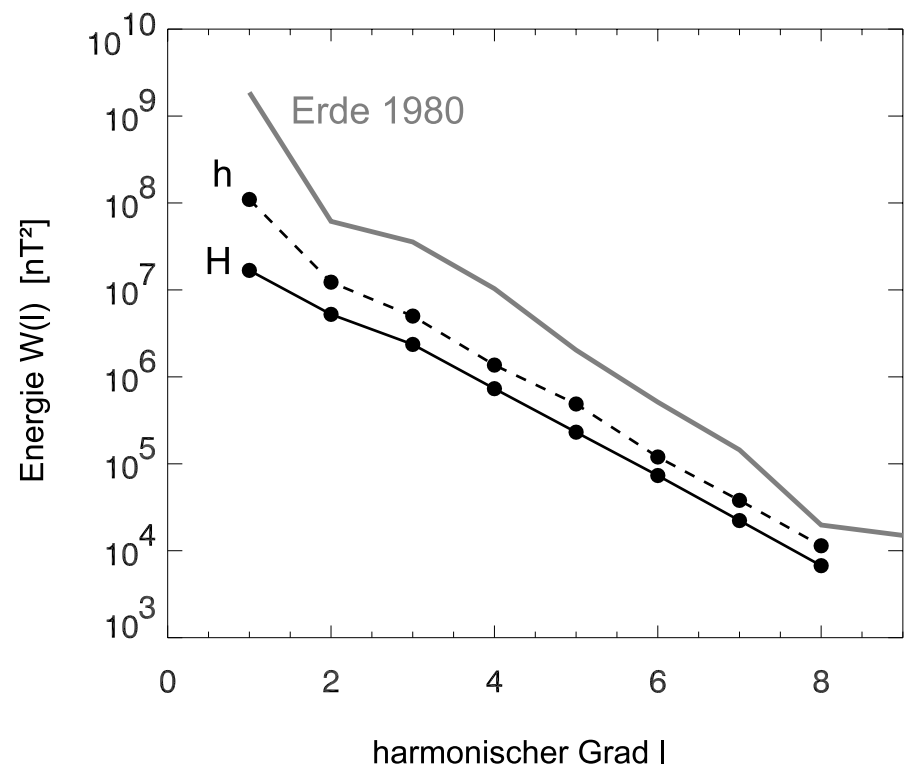

Abb. 4.30: Zeitgemittelte Oberflächenspektren der Modelle h (gestrichelt) und H (durchgezogen). Grau: Spektrum des Erdmagnetfeldes 1980 zum Vergleich. Die Zeitmittelung erfolgte über 1.4 magnetische Diffusionszeiten für das Modell $\mathrm{H}$ und über 0.5 Diffusionszeiten für das Modell h.

die zeitgemittelten Spektren des Magnetfeldes dieser Modelle an der Oberfläche gezeigt. Die Oberflächen-Magnetfelder beider Modelle sind im Mittel dipol-dominiert, wenn auch etwas weniger als bei Modell g (s. Abb. 4.17). Das Modell H mit der größeren Amplitude der Wärmeflußvariation weist den kleineren Dipolbeitrag auf.

Das Modell h konnte leider nur für 0.8 magnetische Diffusionszeiten integriert werden. Während dieser Zeit operiert der Dynamo in einer Polarität und es gibt keine Umkehrungen. Trotzdem erkennt man in der in Abb. 4.31 gezeigten VGP-Verteilung bei etwa $0^{\circ}$ und $180^{\circ}$ Minima und dazwischen Maxima. Wie bei g korrelieren diese mit den Minima und Maxima der Wärmeflußverteilung in äquatornähe (vgl. Abb. 4.29). Wieder können auch in Zeiten ohne Polaritätswechsel VGP-Häufungen beobachtet werden. Dies wird später noch bei Modell g zu sehen sein.

Die Zeitreihe des tomographischen Modells $\mathrm{H}$ ist 2.4 Zeiteinheiten lang und enthält fünf Umkehrungen sowie zahlreiche Exkursionen. Den Großteil der Zeit befindet sich der Dynamo in inverser Polarität, so daß die meisten VGP's in Abb. 4.32 in der Südhemisphäre liegen (unteres Bild). Man erkennt aber im oberen Bild ein ausgeprägtes Maximum bei Breiten von etwa $90^{\circ}$ sowie ein schwächeres bei $-90^{\circ}$, und zusätzlich Minima um $0^{\circ}$ und $180^{\circ}$. Im Bereich des Äquators sind VGP-Durchgänge bei knapp $90^{\circ}$ und $-100^{\circ}$ wahrscheinlicher als bei $0^{\circ}$ und $180^{\circ}$. Die Amplitude der VGP-Kurve ist mit der der Modelle g und G vergleichbar (s. Abb.4.28).

In beiden tomographischen Modellen liegen die virtuellen Pole gehäuft in amerikanischen und ostasiatischen Längen. Für das Modell h ist der Mittelungszeitraum zu kurz, um gesicherte Aussagen über das Histogramm der VGP-Verteilung zu machen. In Modell $\mathrm{H}$ liegen die Maxima der Verteilung der VGP's etwas (ca. $20^{\circ}$ ) nach Westen verschoben, vergleicht 


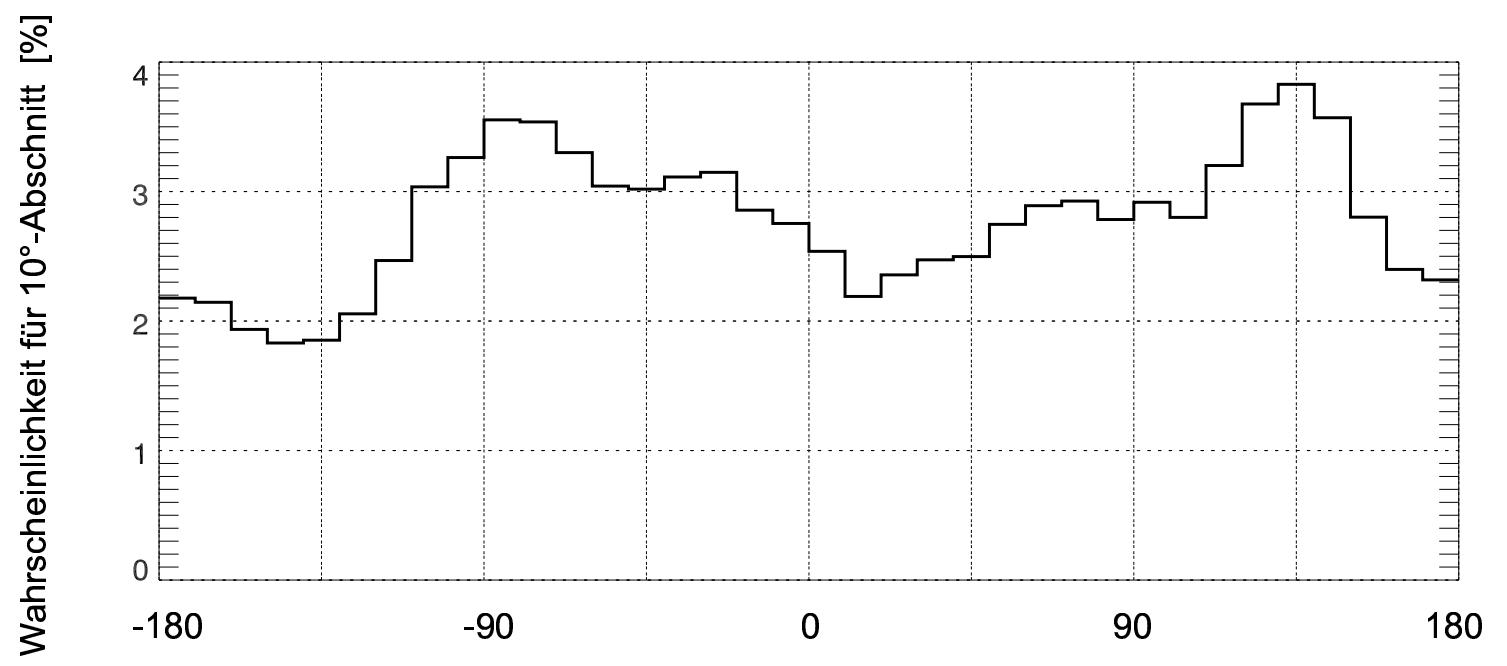

NW- Süd-

Amerika amerika Ostasien

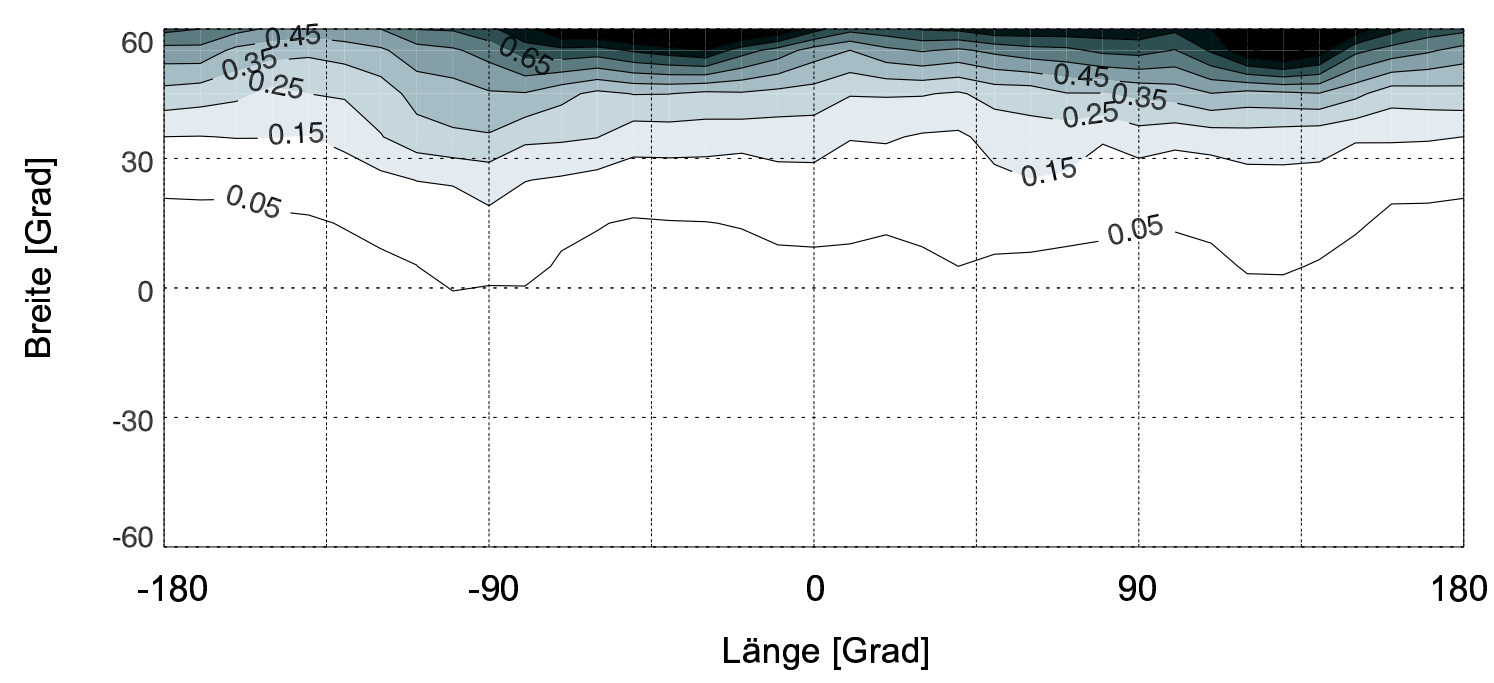

Abb. 4.31: VGP-Dichte für das tomographische Modell h für 2000 zufällige Sites. Mittel über 0.5 magnetische Diffusionszeiten. Oben: Wahrscheinlichkeit für einen VGP, in ein $10^{\circ}$ Längenintervall zu fallen. Unten: Anzahl an VGP's pro $10^{\circ} \times 10^{\circ}$ Intervall, normiert auf 1. Der Konturlinienabstand beträgt 0.1. Die Längen $\phi$ sind hier so gewählt, daß $\phi=0^{\circ}$ dem Greenwich-Meridian entspricht (s. Abb. 4.29). 

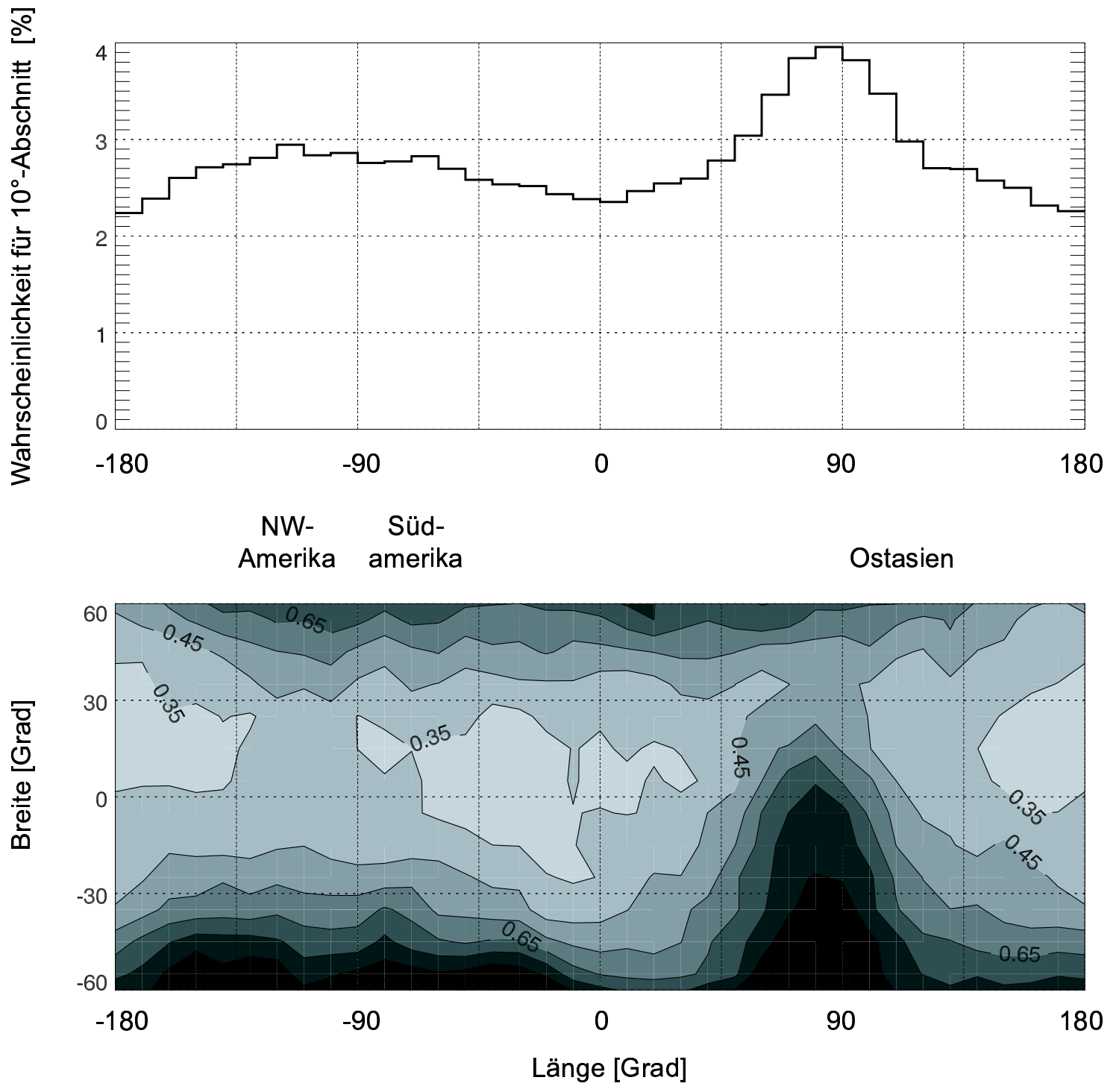

Abb. 4.32: VGP-Dichte für das tomographische Modell H für 500 zufällige Sites. Darstellung wie in Abb.4.31 Mittel über 1.4 magnetische Diffusionszeiten. 
man sie mit den bevorzugten Längen in Clement (1991) (Abb. 4.2) oder Laj et al. (1991) (Abb.4.1).

Gubbins \& Sarson (1994) demonstrierten an ihrem kinematischen Modell, daß sich die VGP's entlang der Längen häufen, in denen starke Flußkonzentrationen zu finden sind. Im Modell von Olson \& Christensen (2002), in welchem dem Dynamo in ähnlicher Weise ein tomographisches Wärmeflußmuster aufgeprägt wird, liegen die Konzentrationen des magnetischen Flusses im Zeitmittel etwas nach Westen versetzt im Vergleich zum historischen (Bloxham \& Jackson, 1992) Erdmagnetfeld. Olson \& Christensen (2002) bemerken aber, daß realistischere Werte der Ekmanzahl diese Konzentrationen weiter nach Osten verschieben. Falls die Häufung der VGP's für die hier simulierten Dynamos auch generell mit den Längen zusammenfällt, in denen im zeitgemittelten Feld Fluß konzentriert ist, so würden bei kleinerer Ekmanzahl die VGP-Häufungen auch etwas weiter nach Osten wandern, und damit besser mit den Beobachtungen übereinstimmen.

VGP's bei Längen von $90^{\circ}$ sind im Modell $\mathrm{H}$ im statistischen Mittel um den Faktor 1.7 häufiger wie VGP's bei Längen von $180^{\circ}$. Betrachtet man einzelne Umkehrungen gesondert, kann dieser Faktor wesentlich größer werden, wie im folgenden Abschnitt dargestellt wird. Für die Matuyama-Brunhes-Umkehr sind VGP's bei ihren bevorzugten Längen etwa 4 mal so häufig wie in Regionen minimaler VGP-Dichte (s. Abb. 4.2).

\subsubsection{VGP-Verteilungen einzelner Umkehrungen}

Es konnte gezeigt werden, daß sich ein longitudinal variierender Wärmefluß in der langzeitig gemittelten Verteilung der VGP's niederschlägt. Betrachten wir jetzt die Verteilung der virtuellen Pole für einzelne Ereignisse (Umkehrungen oder Exkursionen) gesondert. Zwei Fragen sollen beantwortet werden:

- Sieht man im Modell Häufungen der virtuellen Pole für ein einzelnes Ereignis?

- Falls das so ist, liegen die VGP's für ein einzelnes Ereignis ebenfalls häufiger über Regionen hohen Wärmeflusses?

Für die Erde wurde gezeigt, daß Häufungen der VGP's und bevorzugte VGP-Pfade sowohl für eine einzelne Umkehrung auftreten (Clement, 1991; Clement \& Kent, 1991; Hoffman, 2000), als auch wenn man die Daten mehrerer Umkehrungen zusammenfaßt (Love, 1998). Coe et al. (2000) analysieren die VGP-Verteilungen zweier Polumkehrungen, die einem Dynamo-Modell mit KMG-Wärmeflußvariationen entstammen. Es ergibt sich eine sehr grobe Korrelation der Gebiete überdurchschnittlicher VGP-Dichte mit Gebieten hohen Wärmeflusses.

Wir betrachten Umkehrungen des Modells g, dessen Zeitreihe aus Abb. 4.16 bekannt ist und sehen uns die dritte bis sechste Polumkehr um die Zeiten $t_{3}=1.2, t_{4}=2.7, t_{5}=4.2$ und $t_{6}=4.7$ an. Die entsprechenden Abschnitte aus den Zeitreihen der Dipolneigung sind in den Abbildungen 4.33 bis 4.37 jeweils im Teil a) oben dargestellt. Im unteren Bereich der Abbildungen a) ist der relative Dipolanteil an der Erdoberfläche aufgetragen. Dazu wurde nach Formel 4.10 für jeden Zeitpunkt die Energie $W(\ell)$ in den Einzelmoden $\ell=1 \ldots 8$ berechnet (Langel \& Estes, 1982). Dargestellt ist der Dipolanteil $D$ am Oberflächenfeld bis Grad und 
Ordnung 8:

$$
D=\sqrt{\frac{W(\ell=1)}{\sum_{\ell=1}^{8} W(\ell)}}
$$

Für das dipol-dominierte Feld der heutigen Erde ergibt sich

$$
D_{\text {Erde }}=0.97
$$

was auch ein sehr häufiger Wert während stabiler Perioden der Modellrechnungen ist (siehe z. B. den Anfang des Zeitausschnitts der Abb.4.33). Die gleiche Berechnung ist noch einmal für den axialen Dipolanteil $D_{\mathrm{ax}}$ gemacht worden. $D_{\mathrm{ax}}$ erhält man, wenn man in 4.11 statt $W(\ell=1)$,

$$
W(\ell=1)=2\left(\left(g_{1}^{0}\right)^{2}+\left(g_{1}^{1}\right)^{2}+\left(h_{1}^{1}\right)^{2}\right)
$$

nur den axialen Dipolbeitrag $W_{\mathrm{ax}}$ in 4.11 einsetzt,

$$
W_{\mathrm{ax}}=W(\ell=1, m=0)=2\left(g_{1}^{0}\right)^{2}
$$

Beim Übergang von der einen in die andere Polarität muß $D_{\mathrm{ax}}$ mindestens einmal Null werden. Eine größere Differenz zwischen $D$ und $D_{\mathrm{ax}}$ während der Umkehr deutet auf einen signifikanten äquatorialen Dipol hin.

Betrachtet man den ganzen Zeitausschnitt $t=1.05$ bis $t=1.28$ der dritten Polumkehr, der in Abb. 4.33 a) mit vertikalen Linien markiert ist, so läßt sich in der VGP-Verteilung, b), keine klare Systematik erkennen. Bis auf einen Bereich um $150^{\circ}$ Länge in der Nordhemisphäre liegen sowohl die virtuellen als als auch die wahren geomagnetischen Pole überall verstreut. Als wahrer geomagnetischer Pol (kurz TGP) wird hier die Position des Südpols des Dipolfeldes $(\ell=1)$ bezeichnet. Der TGP ist somit unabhängig von der Site; er kann aber im Paläomagnetismus i. a. nicht bestimmt werden, weil der Dipolterm dort nicht von den Feldbeiträgen höherer Multipole zu trennen ist.

Die Feldumkehr läßt sich aufteilen in einen ersten Zeitabschnitt bis $t=1.18$, in dem wenn man die Neigung der Dipolachse zugrundelegt - der eigentliche Wechsel der Polarität stattfindet, und in einen darauf folgenden Zeitabschnitt, in dem das Feld eine Exkursion durchmacht. In Abb. 4.34 sind die VGP-Verteilungen dieser Abschnitte gesondert gezeigt. Während der eigentlichen Umkehr (a) liegen die VGP's gehäuft bei Längen um $0^{\circ}$ und nur sehr wenige fallen in den Längenabschnitt zwischen $180^{\circ}$ und $270^{\circ}$. Am Äquator bei Längen von $315^{\circ}-90^{\circ}$ (dunkler Bereich innerhalb der 0.45-Konturlinie) ist die VGP-Dichte über 5-mal so hoch wie bei Längen um $225^{\circ}$ (weißer Bereich außerhalb der 0.15-Konturlinie). Der axiale Dipolbeitrag sinkt in dieser Zeit teilweise auf unter 0.2, wobei der gesamte Dipolbeitrag selten unter 0.4 sinkt. Zwischen $t=1.08$ und $t=1.10$ ist z. B. $D_{\mathrm{ax}} \approx 0.1$, allerdings $D \approx 0.6$, es gibt also einen signifikanten äquatorialen Dipol, der die Häufung der VGP's um die tatsächlichen Pol-Lagen bedingt.

Bei der auf die Umkehr folgenden Exkursion (b) liegen die VGP's fast ausschließlich in der Südhemisphäre und sammeln sich bei Längen um $235^{\circ}$, also zwischen Maximum und Minimum des Wärmeflusses.

Die vierte Polumkehr (4.35) ist zeitlich besser einzugrenzen und hat eine Dauer von etwa 0.06 Diffusionszeiten, wenn man den Polaritätswechsel an der Zeitreihe der Dipolneigung festmacht. Betrachten wir die VGP-Verteilung während der Umkehr zwischen $t=2.68$ und 


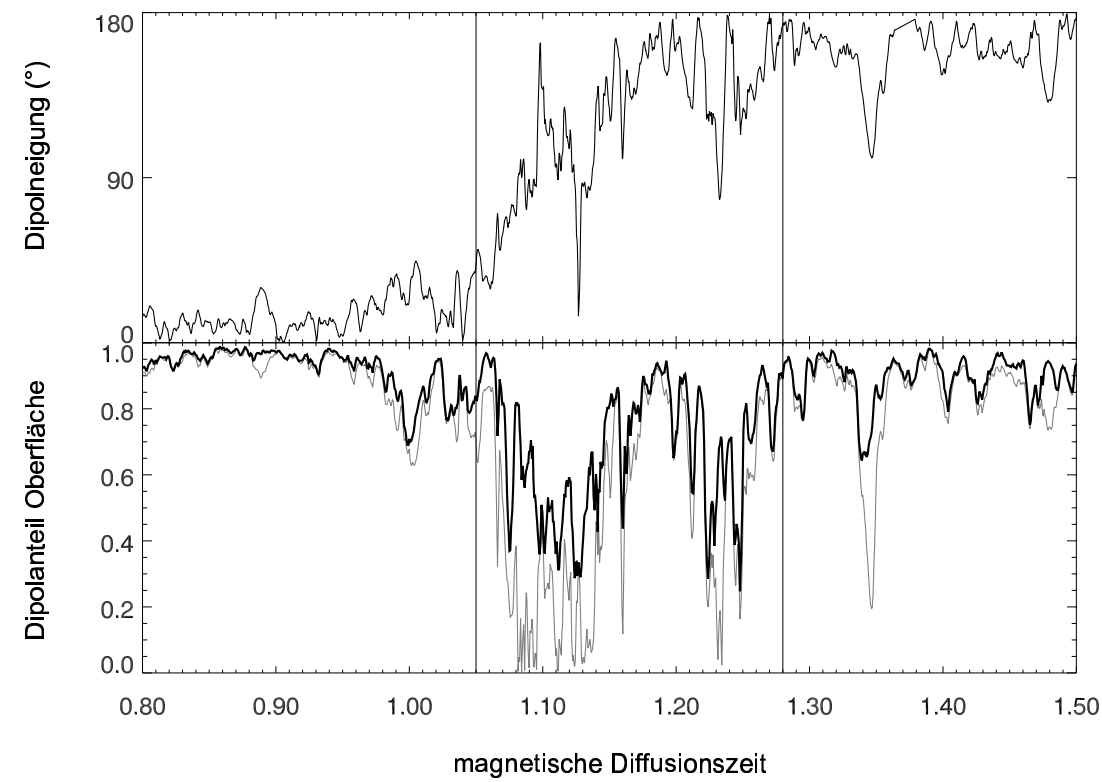

a)

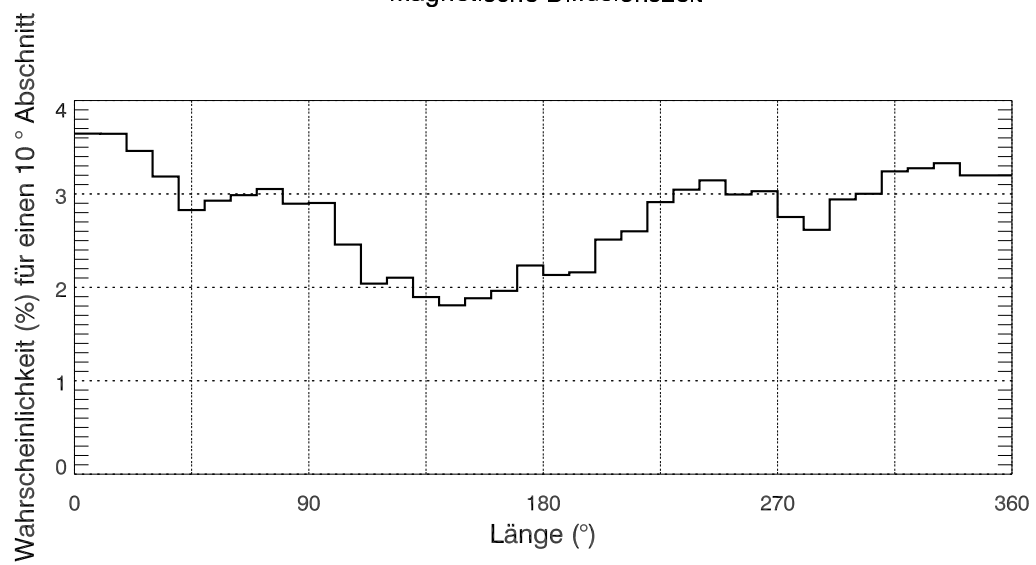

b)

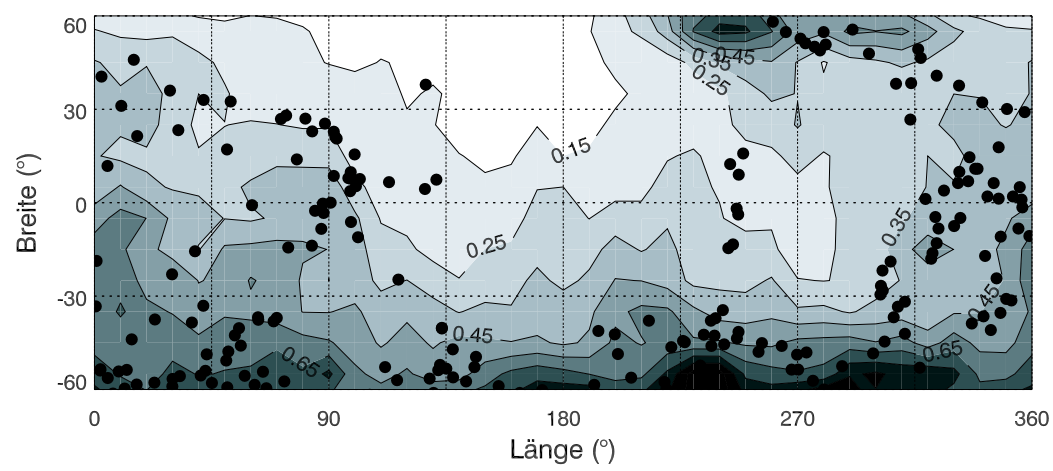

Abb. 4.33: Dritte Polumkehr von Modell g. In Bild a) ist gezeigt: oben die Dipolneigung und unten der Dipolbeitrag $D$ an der Oberfläche nach 4.11 (schwarze Linie) sowie der Beitrag $D_{\text {ax }}$ des axialen Dipolfeldes zum Oberflächenfeld (graue Linie). In Bild b) ist die VGP-Dichte für 2000 zufällige Sites gezeigt. (Gleiche Darstellung wie in Abb. 4.31). Die Punkte geben die Lage der wahren geomagnetischen Pole (TGP's) im betrachteten Zeitintervall an. 


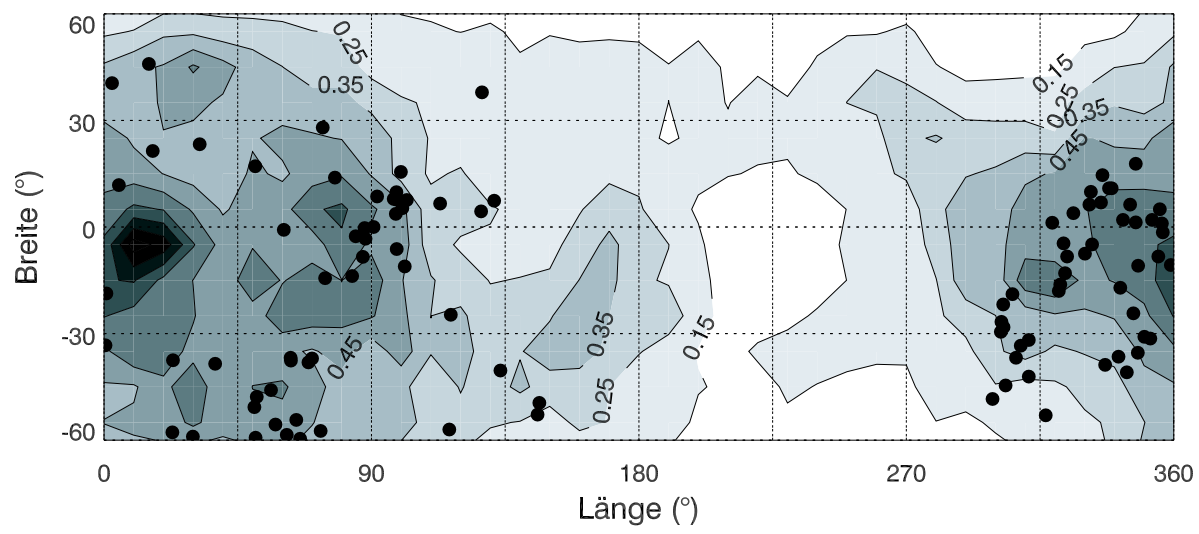

(a) Zeitraum der eigentlichen Polumkehr, $t=1.08-1.15$.

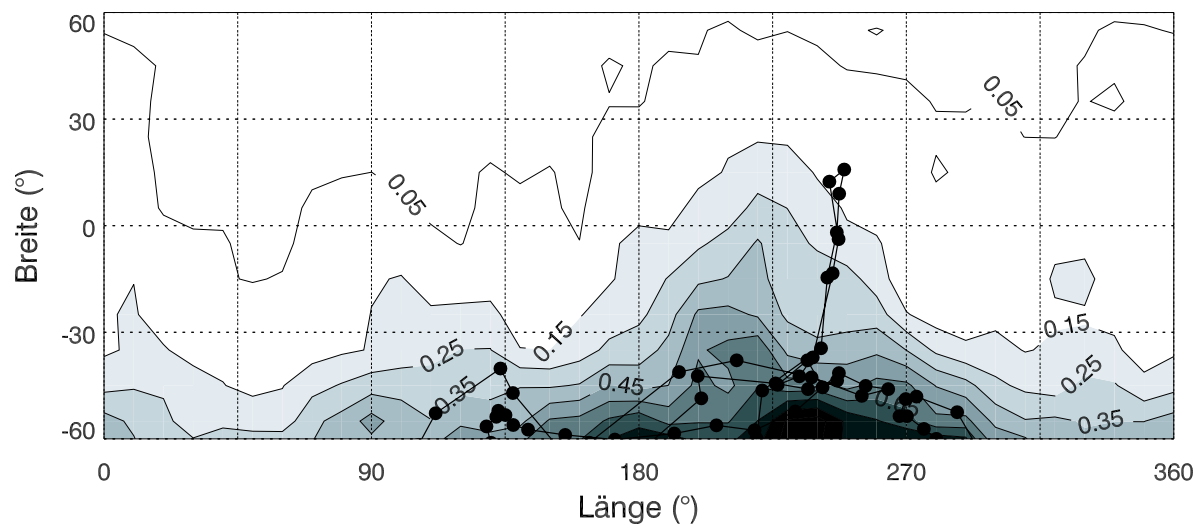

(b) Zeitraum der folgenden Exkursion, $t=1.18-1.28$. Zeitlich aufeinander folgende TGP's sind mit einer Linie verbunden.

Abb. 4.34: Dritte Polumkehr von Modell g. VGP-Dichte für 2000 zufällige Sites. Die Punkte geben die Lage der wahren geomagnetischen Pole (TGP's) an. 


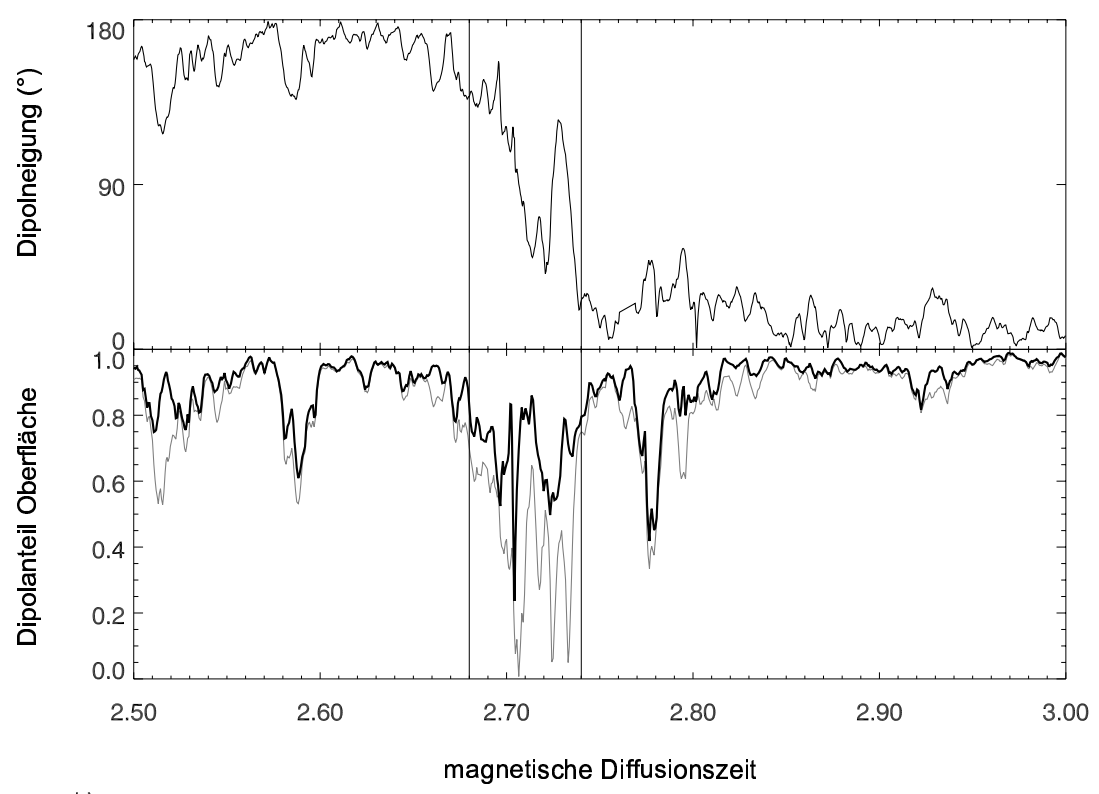

a)

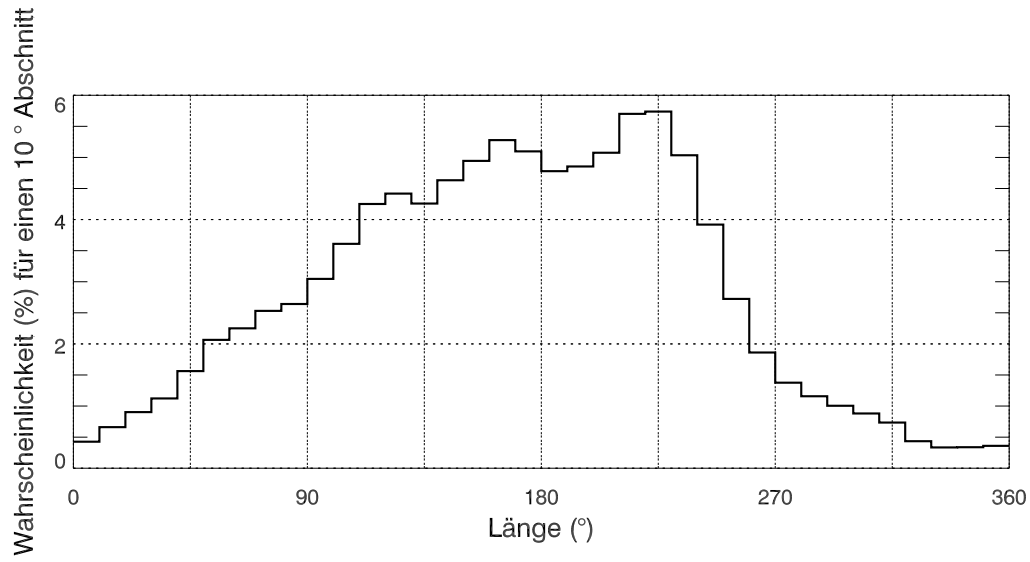

b)

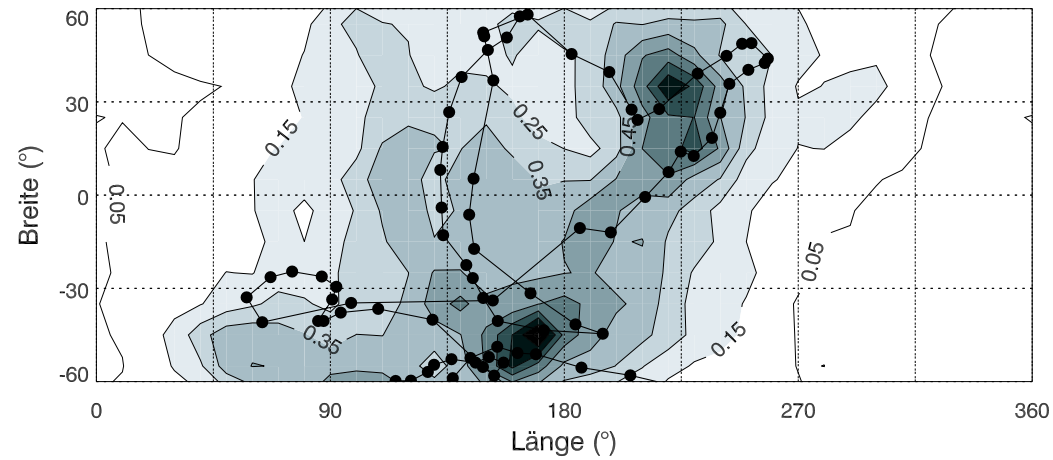

Abb. 4.35: Vierte Polumkehr von Modell g. Darstellung wie in Abb.4.33. 
$t=2.74$, so gibt es ein breites Maximum bei Längen von $180^{\circ}$ und ein ausgeprägtes Minimum bei $0^{\circ}$ (Abb. b). Zusätzlich häufen sich die VGP's an zwei Punkten mittlerer Breiten (Abb. b, unten). Auch hier gibt es während der Umkehr Zeiträume, in denen der axiale Dipolbeitrag viel kleiner ist als der gesamte Dipolbeitrag. Während der Umkehr ist an der Oberfläche ein signifikanter (äquatorialer) Dipol vorhanden, so daß sich die VGP's um die Lage der TGP's häufen.

Bei der fünften Umkehr (Abb. 4.36) findet sich für das Zeitintervall $t=4.19$ bis $t=4.24$ eine extreme Häufung der VGP's in einem schmalen Längenband um $180^{\circ}$. Für diese Umkehrung ist die Differenz zwischen Dipolbeitrag $D \approx 0.65$ und axialem Dipolbeitrag $D_{\text {ax }} \approx 0.20$ größer als bei den beiden bisher betrachteten Umkehrungen. Der starke äquatoriale Dipol führt dazu, daß VGP's bei $180^{\circ}$ Länge etwa 10 mal so häufig sind, wie um $0^{\circ}$ (Abb. b). In diesem Fall geben die virtuellen Pole recht genau die jeweilige Lage des wahren Pols an. Auch hier gibt es nach der eigentlichen Umkehr wieder eine Exkursion bei $t=4.28$, bei der zeitweise ein äquatorialer Dipol das Oberflächenfeld beherrscht. Während dieser Exkursion liegen die VGP's gehäuft im Längenintervall zwischen $90^{\circ}$ und $135^{\circ}$ (nicht gezeigt).

Die sechste Umkehr (Abb. 4.37a) dauert etwa 0.2 Diffusionszeiten und damit viel länger als die anderen; hier gibt es einen lang andauernden Zeitraum mit schwankender Dipolrichtung und unterdurchschnittlich kleinem Dipolbeitrag zum Oberflächenfeld. Die VGPVerteilung, b), zeigt eine Häufung bei Längen um $200^{\circ}$ und eine weitere, schwächer ausgeprägte bei $0^{\circ}$. Desweiteren ist ein deutliches Minimum bei $90^{\circ}$ Länge zu erkennen. Diese VGP-Verteilung besitzt eine leichte Ähnlichkeit zu der von Hoffman (2000) für die Matuyama-Brunhes Polumkehr abgeleiteten Häufigkeitsverteilung (Abb. 4.3). Dort gibt es zwei Maxima mit knapp $180^{\circ}$ Abstand in der Länge, und die meisten VGP's befinden sich in hohen Breiten. Im Fall des Modells entstehen die Häufungen in der VGP-Verteilung an verschiedenen Orten zeitlich nacheinander.

Für die zwei Umkehrungen des Modells G mit der höheren Amplitude werden in einem Fall VGP-Längen um $180^{\circ}$ bevorzugt (ähnlich wie in Abb. 4.35), im anderen Fall aber sind die VGP's über die Längenintervalle etwa gleichverteilt.

Um eine Häufung von VGP's während einer Umkehr zu sehen, reicht schon eine geringe Anzahl an Sites aus. Für die vierte Polumkehr wird die gleiche Auftragung, wie sie in Bild 4.35 für 2000 Sites gezeigt ist, noch einmal für eine zufällige Wahl von 5 Sites vorgenommen. Da aufgrund der geringen Anzahl der Sites viel weniger VGP's vorliegen, werden diese in $15^{\circ}$-Abschnitte einsortiert. Um die Dispersionskurve aus Abb. 4.35 mit Abb. 4.38 zu vergleichen, muß erstere mit 1.5 multipliziert werden. Das Maximum in Abb. 4.35 bei knapp $6^{\circ}$ übersetzt sich dann in einen Wert von knapp $9^{\circ}$. Die Maxima der Dispersionskurve für 5 und für 20 Sites (graue Kurve) liegen bei etwa $11^{\circ}$ bzw. $8^{\circ}$, was bezogen auf die geringere Anzahl an VGP's eine akzeptable Übereinstimmung ist. Vergleicht man die unteren Bilder von Abb. 4.35 und Abb. 4.38 miteinander, so sieht man, daß auch im Falle von nur 5 Sites die VGP-Häufigkeitsverteilung überraschend gut wiedergegeben wird. Das gleiche ist noch einmal am Beispiel der fünften Polumkehr in Abb. 4.39 dargestellt. Auch im Fall von nur 5 Sites ist die Häufung der virtuellen Pole klar zu erkennen.

Zusammenfassend kann gesagt werden, daß sich für die durchschnittlich 0.06 Diffusionszeiten (entsprechend 9000 Jahren) langen Intervalle der ersten drei Umkehrungen deutliche Häufungen der virtuellen Pole ergeben. Die Häufungen liegen in allen drei Fällen über ei- 


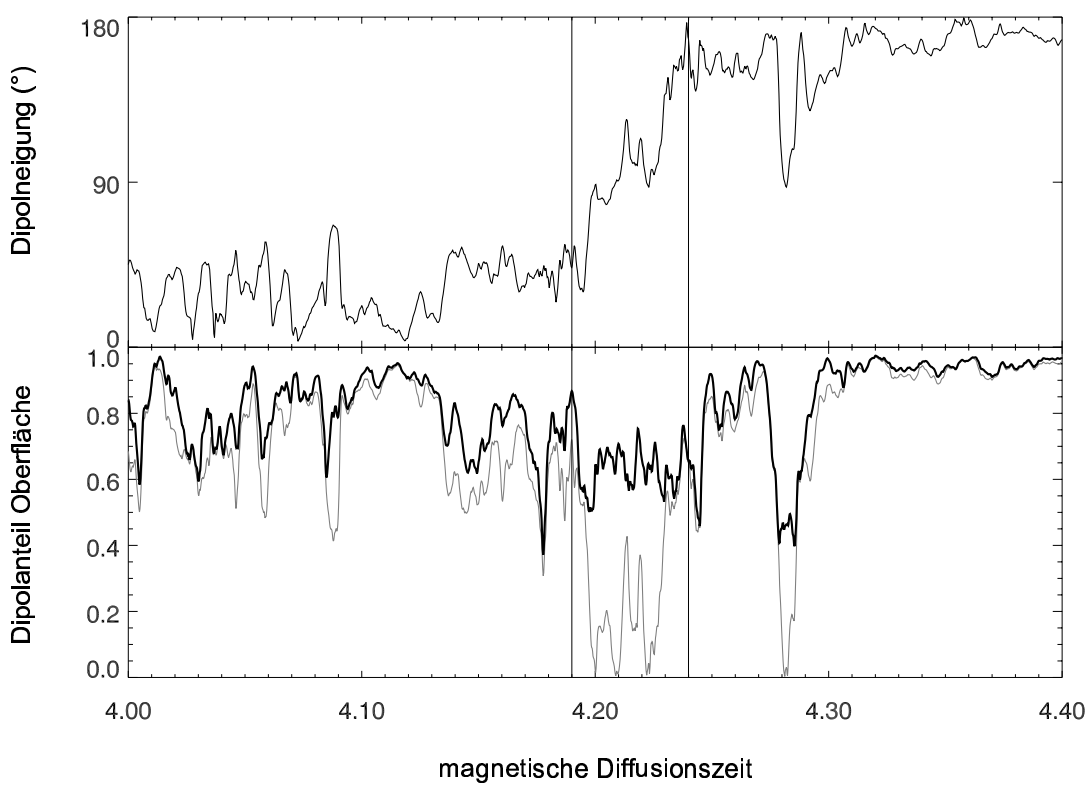

a)

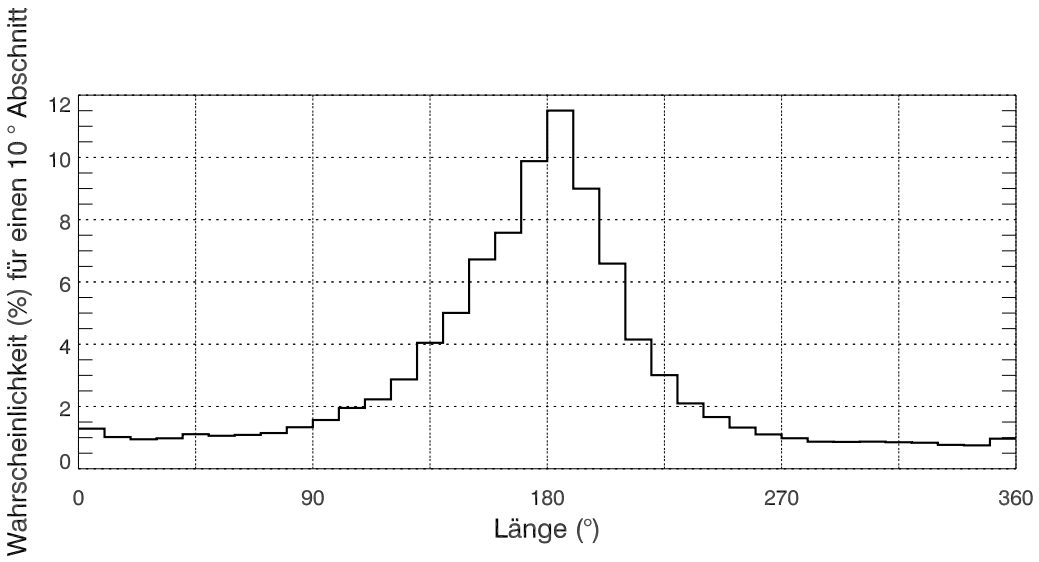

b)

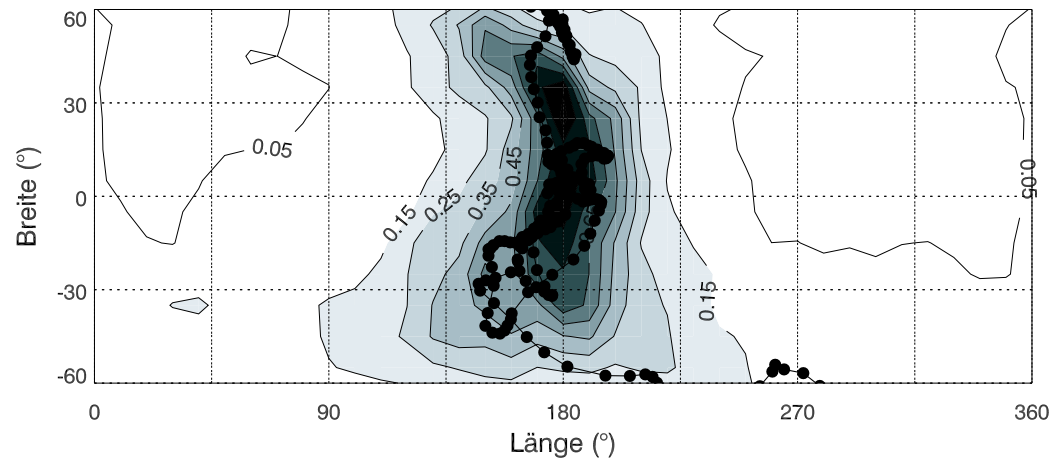

Abb. 4.36: Fünfte Polumkehr von Modell g. Darstellung wie in Abb. 4.33 


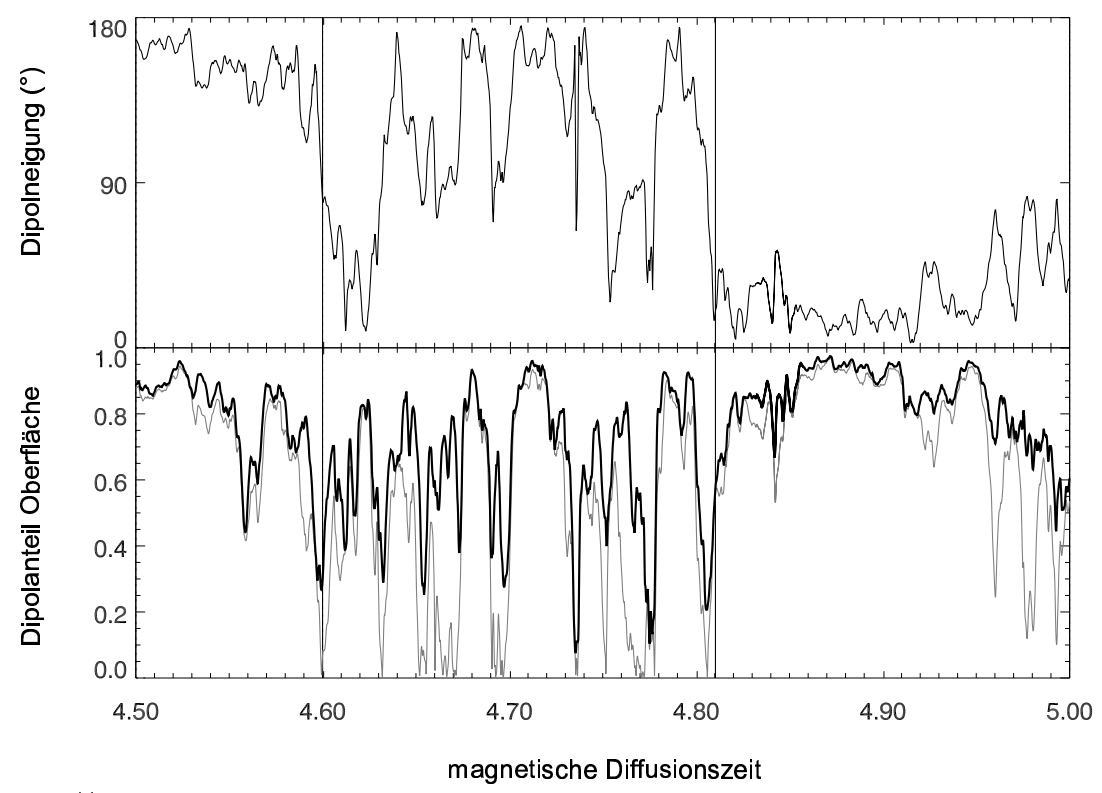

a)

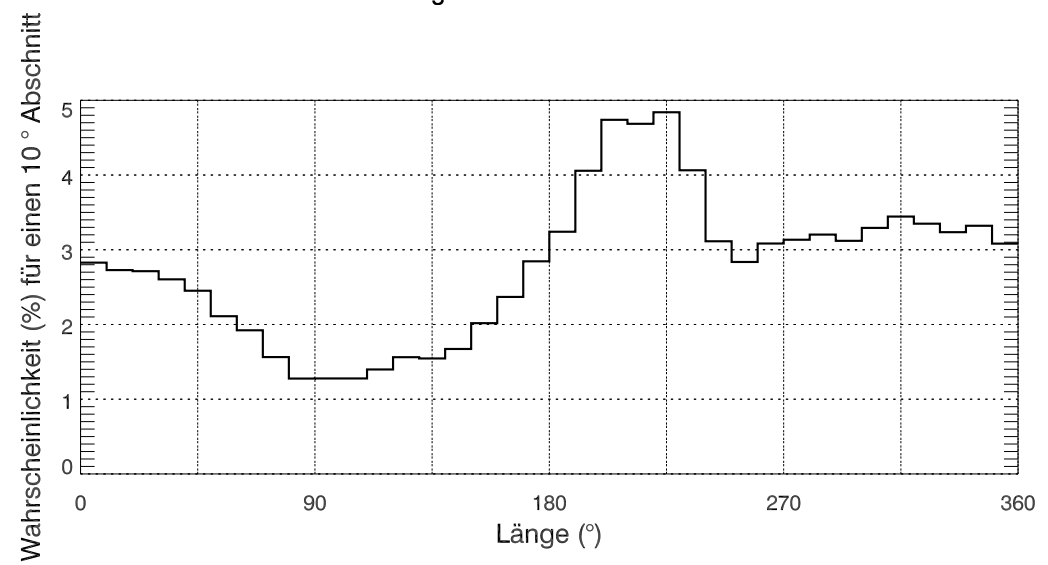

b)

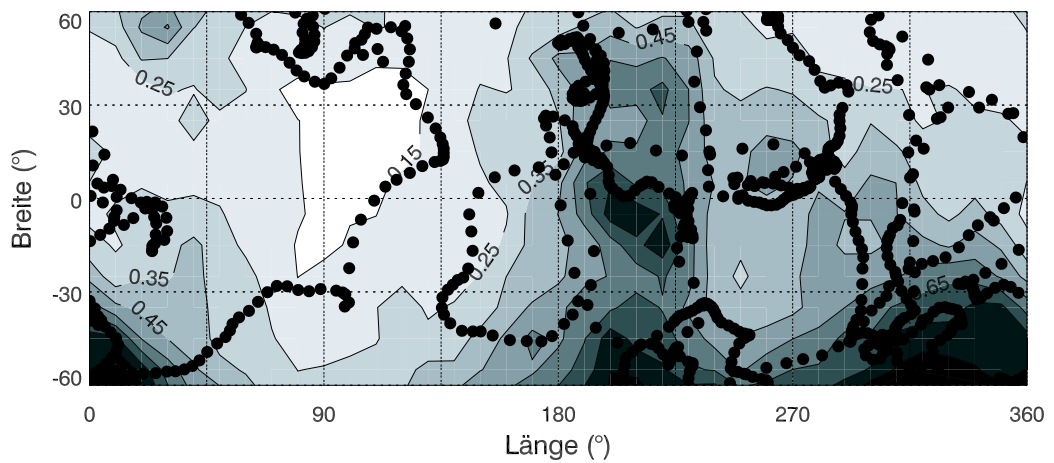

Abb. 4.37: Sechste Umkehr von Modell g. Darstellung wie in Abb.4.33 aber für 500 Sites. 

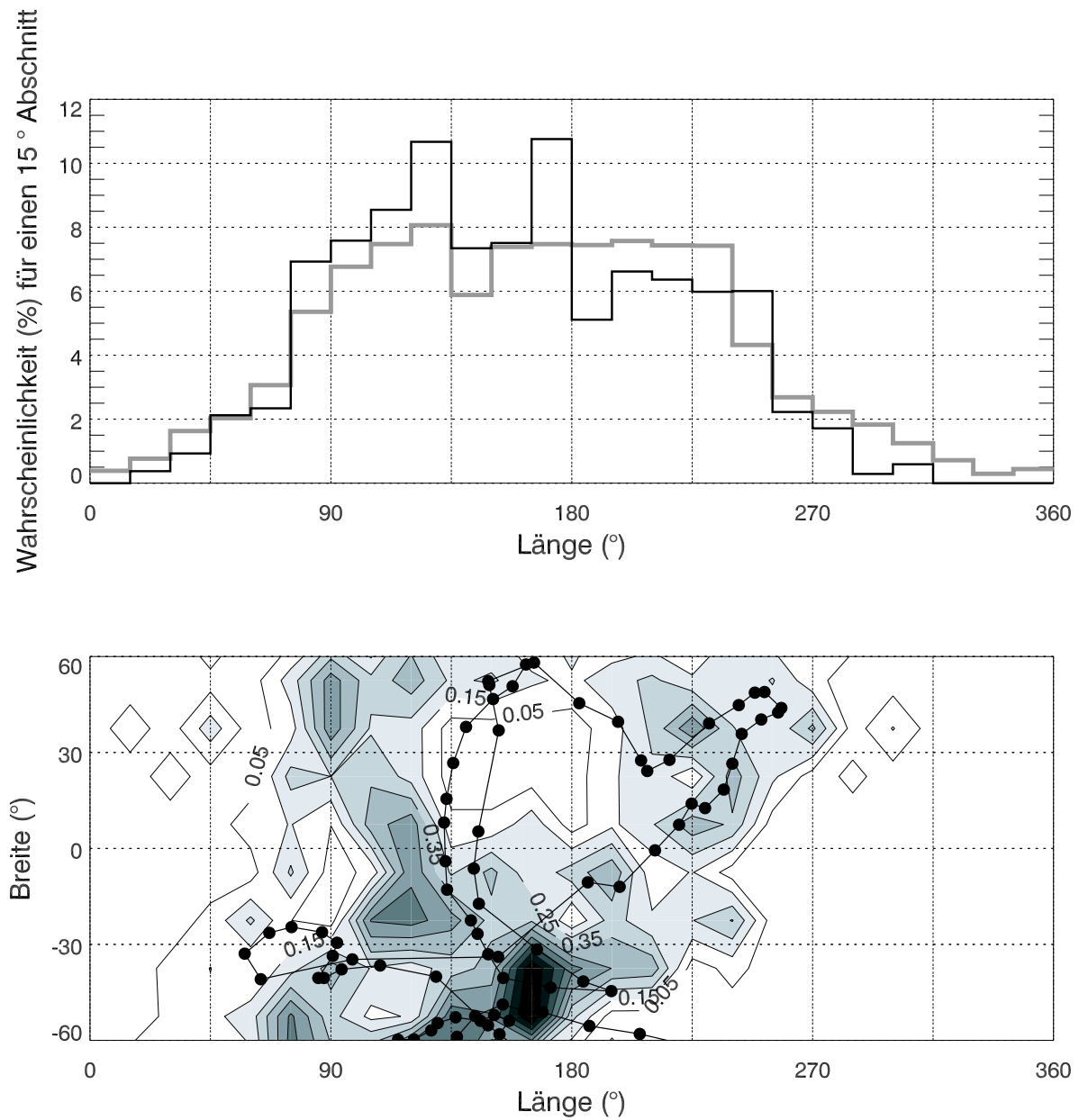

Abb. 4.38: Vierte Polumkehr von Modell g. Gleiche Darstellung wie in Abb. 4.35 b), aber für $15^{\circ}$-Abschnitte und 5 zufällige Sites. Die graue Kurve im oberen Bild gibt zusätzlich die Dispersionskurve für 20 zufällige Sites an. 

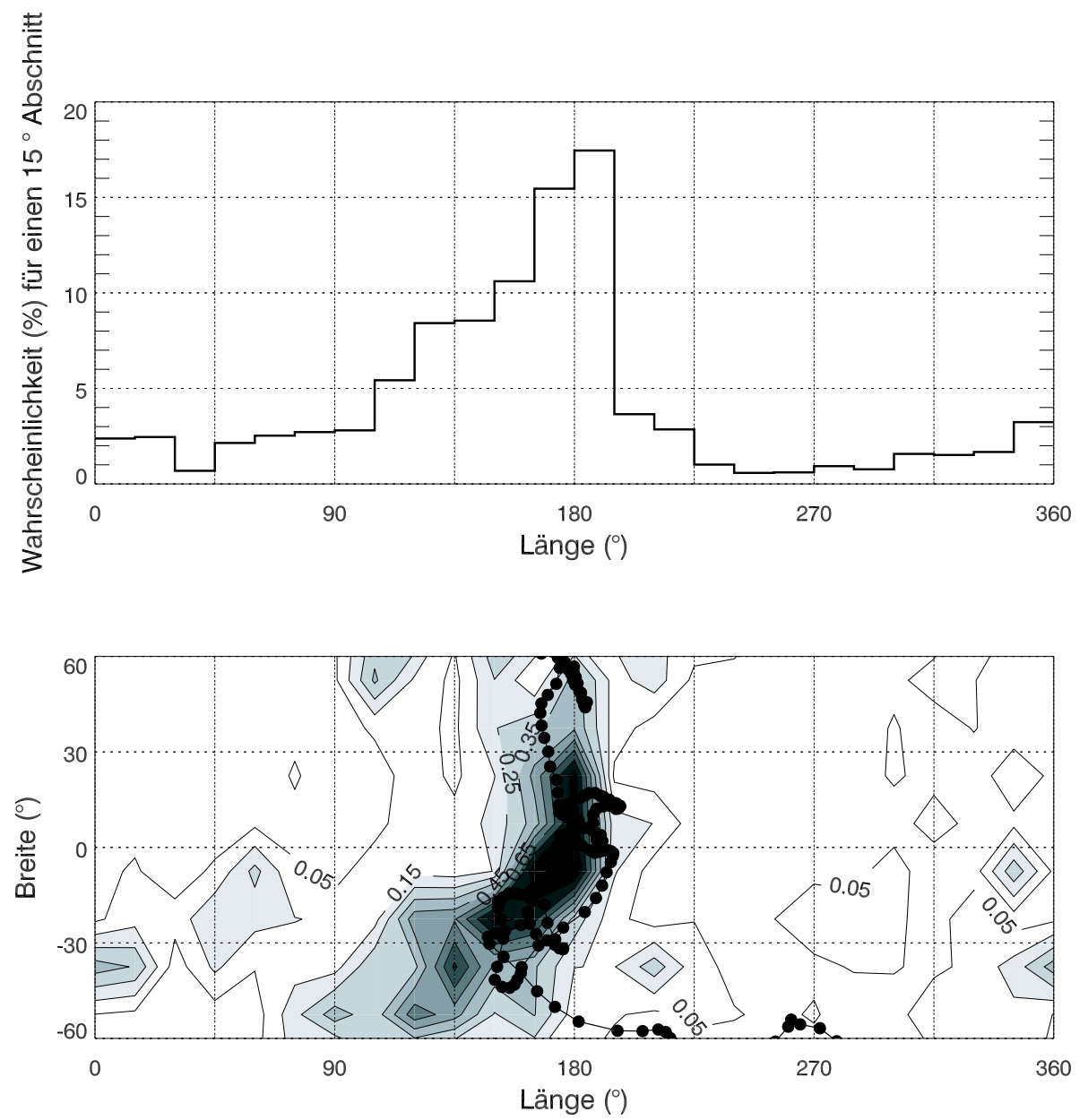

Abb. 4.39: Fünfte Polumkehr von Modell g. Gleiche Darstellung wie in Abb.4.36b), jedoch für 5 zufällige Sites und $15^{\circ}$-Abschnitte. 
nem Maximum des KMG-Wärmeflußmusters, was allerdings auch Zufall sein könnte. In zwei Fällen ist das Maximum der VGP-Verteilung in der Länge recht breit, in einem Fall aber ist es stark lokalisiert. Nur in einer Umkehr, die mit 0.2 Diffusionszeiten (entsprechend 30000 Jahren) wesentlich länger dauert, als die anderen, finden sich zwei Maxima in der Länge, ähnlich wie bei der Matuyama-Brunhes-Polumkehr (Hoffman (2000), Abb.4.3). Abschätzungen der Länge der Matuyama-Brunhes-Umkehr liegen etwa zwischen 2500 Jahren (Love \& Mazaud, 1997) und 12000 Jahren (Singer \& Pringle, 1996). Nur in einem Fall wird von einer sehr langen Dauer einer Umkehr des Erdmagnetfeldes berichtet: Herrero-Bervera et al. (1987) schätzen die Dauer der älteren Olduvai-Polumkehr auf 28000 Jahre ab.

Häufungen der virtuellen Pole sind schon gut erkennbar, wenn man diese für 5 Sites bestimmt.

\subsection{Einfluß des KMG-Wärmeflußmusters auf die Umkehrfrequenz}

Für die Modelle $b$ bis $f$ konnten keine offensichtlichen Unterschiede der VGPBreitenverteilungen entdeckt werden, welche auf die Breitenabhängigkeit des KMGWärmeflusses zurückzuführen wären. Die Breitenverteilung der VGP's ist für jedes Modell zwar leicht unterschiedlich, sie hängt aber hauptsächlich davon ab, wie oft das Modell umkehrt. Ein Beispiel war in Abb.4.15gezeigt. Häufige Umkehrungen oder Exkursionen führen zu einer erhöhten Dichte von VGP's in äquatorialen Regionen. Falls die VGP-Dichte auch über Breiten hohen KMG-Wärmeflusses erhöht sein sollte, dann ist dieser Effekt zu klein, um in den Modellen sichtbar zu werden.

Nachdem gezeigt wurde, daß das Wärmeflußmuster an der Kern-Mantel-Grenze zu Häufungen der virtuellen Pole führt, soll noch sein Einfluß auf die Umkehrfrequenz untersucht werden. Die Auswirkung verschiedener an der KMG vorgegebener Wärmeflußmuster auf dreidimensionale, selbstkonsistente Dynamosimulationen wurde erstmals von Glatzmaier et al. (1999) (im folgenden kurz G99) untersucht. Dort werden allerdings skalenabhängige Werte der Diffusivitäten verwendet, welche zu künstlichen Umkehrungen führen können, wie Grote et al. (2000a) zeigten. Durch die Hyperdiffusivitäten wird Energie auf kleinen Skalen stark gedämpft, so daß G99 nur eine numerische Auflösung bis zum Kugelfunktionsgrad $\ell_{\max }=21$ verwenden. Im Gegensatz dazu werden die hier vorgestellten Modelle bis $\ell_{\max }=85$ entwickelt. Dies ist erforderlich, da gerade das magnetische Spektrum noch viel Energie auf kleinen Skalen enthält. Das Maximum der magnetischen Energiedichte in der Kugelschale ist für die umkehrenden Dynamos bei $\mathrm{E}=3 \times 10^{-4}$ typischerweise recht breit und liegt bei Kugelfunktionsgraden von $\ell_{\max }=5 \ldots 15$ (vgl. dazu Abb. 2.2 auf S. 18).

G99 benutzen einen mittleren Wärmefluß durch die KMG, der 1.44-fach überadiabatisch ist. Während der mittlere überadiabatische Wärmefluß etwa $0.015 \mathrm{~W} / \mathrm{m}^{2}$ beträgt, wird die Amplitude der Wärmeflußvariation $\mathrm{zu} \pm 0.045 \mathrm{~W} / \mathrm{m}^{2}$ gewählt, so daß es auch Bereiche unteradiabatischen Wärmeflusses gibt. Als Ergebnis ihrer Simulationen finden sie eine starke Abhängigkeit des Umkehrverhaltens und des mittleren Dipolmomentes von der Geometrie des aufgeprägten Wärmeflusses. Aufgrund der Tatsache, daß ihr Modell mit homogenem Wärmefluß das ,erdähnlichste“ Verhalten zeigt (in Bezug auf die Umkehrfrequenz und das mittlere Dipolmoment), schließen sie, daß die Wärmeflußvariationen an der KMG nicht sehr 
a
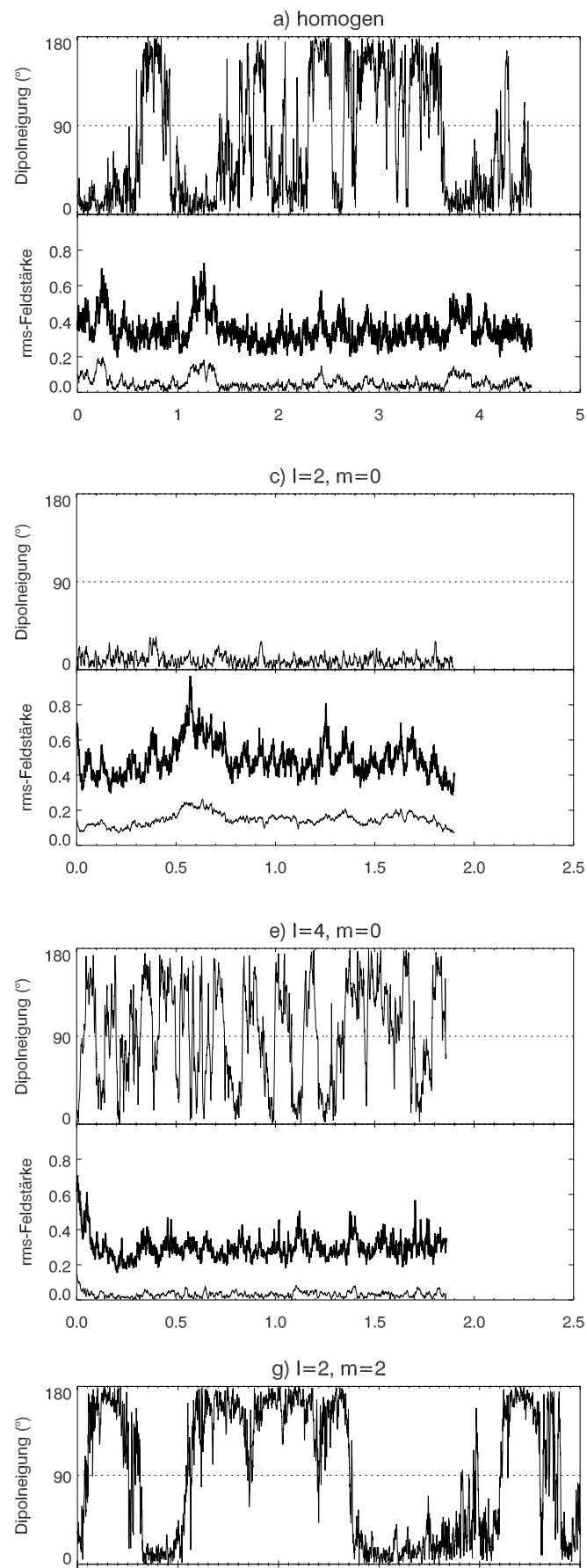

g

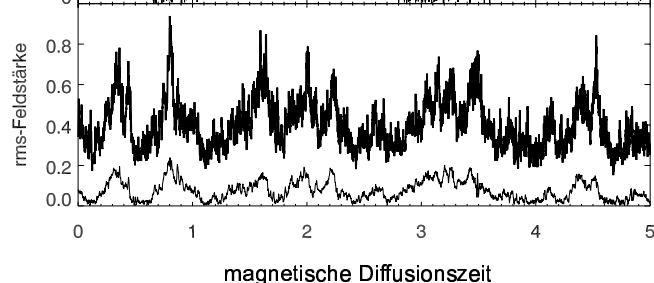

b
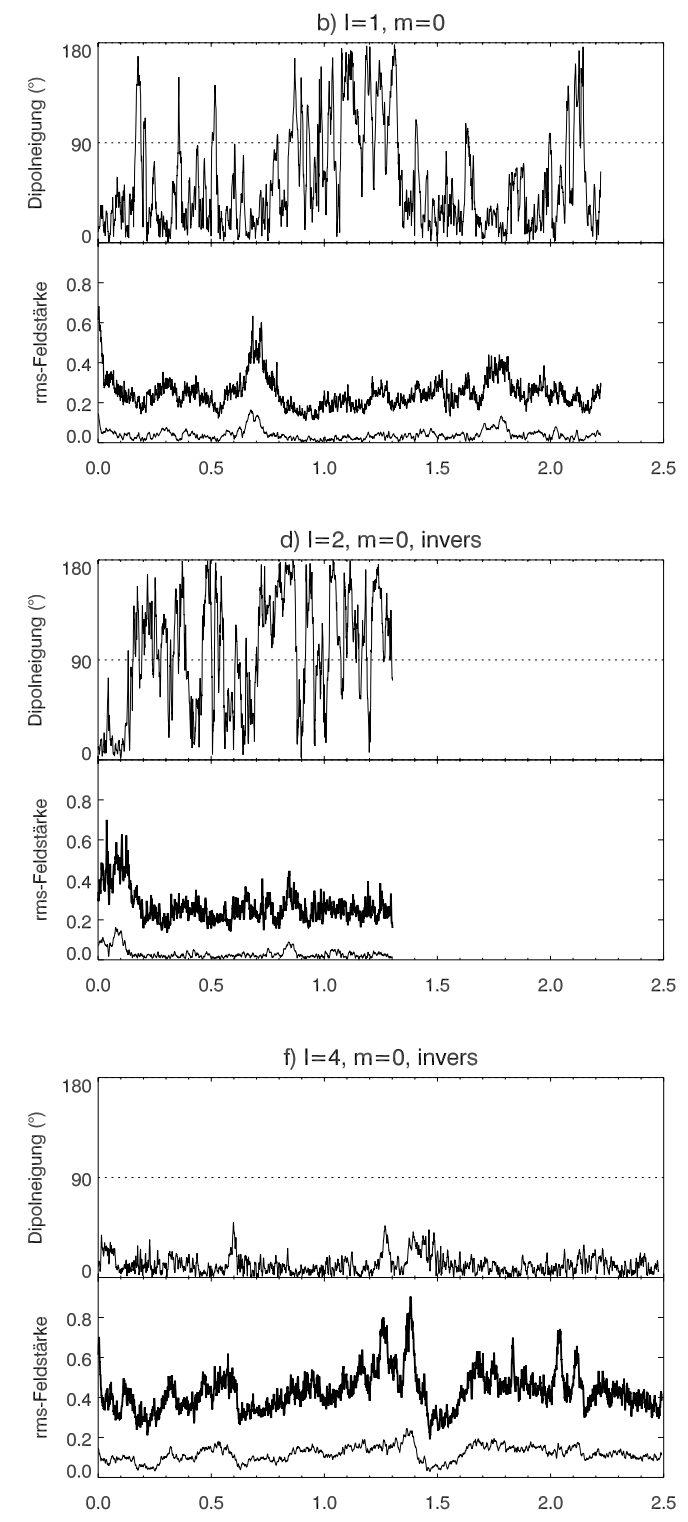

$\mathrm{H}$

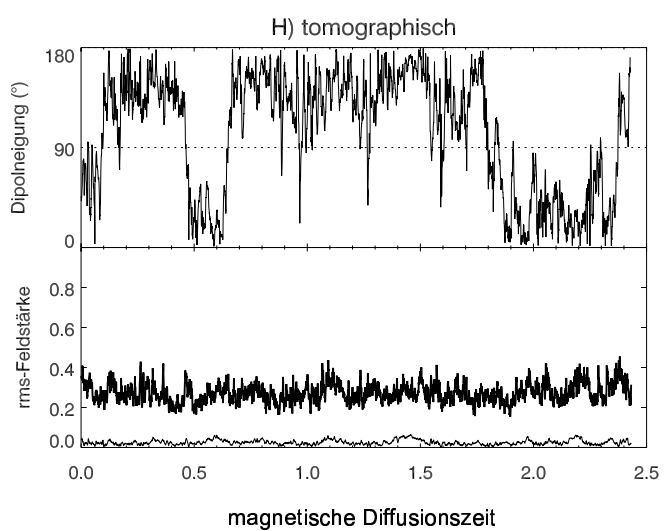

Abb. 4.40: Vergleich 8 verschiedener KMG-Wärmeflußmuster. Jeweils oben: Dipolneigung; unten: rms-Stärke des gesamten KMG-Feldes (dicke Linie) und des Dipolfeldes (dünne Linie). Die Zeitskala in a und $g$ ist doppelt so lang wie in den anderen Fällen. 
groß sein können. Die Variationen der seismischen Geschwindigkeit im unteren Mantel seien deswegen eher auf eine geänderte chemische Zusammensetzung des Mantelgesteins als auf die Temperatur zurückzuführen.

Für acht der im Rahmen dieser Untersuchung simulierten Modelle (Tab. 4.2 auf Seite 63) ist in Abb.4.40 jeweils im oberen Graphen die Neigung der Dipolachse gegen die Zeit aufgetragen. Dabei entspricht eine magnetische Diffusionszeit etwa 150000 Jahren. Die zugehörigen Wärmeflußmuster sind in Abb. 4.4 auf Seite 49 in gleicher Anordnung gezeigt. Die Amplitude der Wärmeflußvariation ist in den Modellen b bis g gleich groß.

Abhängig vom aufgeprägten Wärmeflußmuster gibt es starke Unterschiede in der Umpolhäufigkeit der Dynamos. Während die Modelle a (homogener Wärmefluß), g (Variation in der $(\ell=2, m=2)$-Mode) und $\mathrm{H}$ (tomographischer Wärmefluß) 6-12 Umkehrungen pro Million Jahre hervorbringen, sind es in den Modellen d und e viel mehr, nämlich grob abgeschätzt 50 pro Million Jahre. Die Modelle $c$ und $f$ bleiben dagegen während der ganzen 300000 bzw. 375000 Jahre der Simulation in einer Polarität.

Ein weiterer Unterschied zwischen den Modellen ist die mittlere Schwankung der Dipolneigung in Zeiten stabiler Polarität. Sie soll in diesem Zusammenhang als Säkularvariation bezeichnet werden. In den nicht umkehrenden Modellen $c$ und $f$ ist sie verhältnismäßig klein. Die mittlere Dipolneigung beträgt in beiden Fällen etwa $10^{\circ}$ (s. Tab. 4.2). In den Modellen $\mathrm{b}$ (Variation in der $(\ell=1, m=0)$-Mode) und $\mathrm{H}$ ist sie groß und Neigungswinkel über $20^{\circ}$ sind häufig zu beobachten. Das tomographische Modell $\mathrm{H}$ hat im Vergleich zum Modell $\mathrm{g}$ eine größere Säkularvariation. Diese ist allerdings abhängig von der Amplitude der Wärmeflußvariation, welche in $\mathrm{H}$ größer als in $\mathrm{g}$ ist (s. Tab. 4.2). Im tomographischen Modell h mit geringerer Amplitude der Variation ist auch die Säkularvariation kleiner.

In den jeweils unteren Graphen der Abb. 4.40 ist die durchschnittliche Feldstärke an der KMG gezeigt. Die dicke Linie bezeichnet die rms-Gesamtfeldstärke $B_{\mathrm{KMG}}$, die dünne Linie die rms-Feldstärke $B_{\text {dip }}$ des Dipolfeldes allein. Deren Mittelwerte sind in Tab. 4.2 zu finden. Die nicht umkehrenden Modelle $\mathrm{c}$ und $\mathrm{f}$ haben einen durchschnittlichen Dipolbeitrag zum Feld von $\bar{B}_{\mathrm{dip}} / \bar{B}_{\mathrm{KMG}} \approx 30 \%$. Dieser Wert liegt für die umkehrenden Modelle aus Abb. 4.40 zwischen 9\% und 20\%. Ein stabiles (nicht umkehrendes) Magnetfeld korelliert mit einem hohen mittleren Dipolbeitrag. Umgekehrt treten in den Modellen mit niedrigem mittleren Dipolbeitrag Umkehrungen auf.

Diese Korrelation gilt auch für die Dynamos, die im Rahmen der Parameterstudie in Kap. 3.4 untersucht wurden. Die drei in Abb. 3.10 auf Seite 36 gezeigten Dynamos unterscheiden sich nur in ihrer Rayleighzahl. Abhängig von Ra ist entweder der Dipolbeitrag zum Feld hoch und der Dynamo stabil (oberstes Bild, Ra $/ \mathrm{Ra}$ krit $=16, \bar{B}_{\text {dip }} / \bar{B}_{\mathrm{KMG}}=56 \%$ ) oder der Dipolbeitrag niedrig und der Dynamo kehrt um (unterstes Bild, Ra/Rakrit $=31, \bar{B}_{\mathrm{dip}} / \bar{B}_{\mathrm{KMG}}=24 \%$ ). Eine Änderung des KMG-Wärmeflußmusters hat also einen Effekt auf die Umkehrfrequenz, der auch durch Änderung der Rayleighzahl erreichbar ist. Obwohl die Rayleighzahl für den Vergleich der unterschiedlichen Wärmeflußmuster konstant gehalten wurde, kann doch die Konvektion in den Modellen unterschiedlich stark sein.

Durch die Corioliskraft werden bei kleinen Ekmanzahlen Flüssigkeitsbewegungen in zRichtung (also in Richtung der Rotationsachse) erschwert. Für den Grenzfall einer geostrophischen Strömung, in der nur Coriolis- und Druckgradientkräfte wirken, gilt das ProudmanTaylor-Theorem (siehe z. B. Fearn (1998)), welches besagt, daß dann die Ableitung des Geschwindigkeitsfeldes in $z$-Richtung verschwindet: $\partial \mathbf{u} / \partial z=0$. Als Konsequenz bewegt sich 
die Flüssigkeit in Säulen, welche entlang der Rotationsachse ausgedehnt sind (Busse, 1975). In MHD Dynamos kann das Proudman-Taylor-Theorem aus verschiedenen Gründen nicht erfüllt werden. Wegen der geneigten Grenzflächen muß sich $\mathbf{u}$ in $z$-Richtung ändern, wo eine Konvektionssäule auf die KMG trifft. Zusätzlich gibt es neben den Coriolis- und Druckgradientkräften noch andere Kräfte, wie z. B. Auftriebs- und Lorentzkräfte, welche nicht vernachlässigbar sind. Das Theorem hat aber seine Auswirkungen in dem Sinne, daß Änderungen des Geschwindigkeitsfeldes in $z$-Richtung immer geringer werden, je wichtiger die Corioliskräfte sind.

Da die Konvektionsstruktur näherungsweise aus vertikalen Säulen besteht, sind für den Antrieb der Konvektion hauptsächlich die Dichteunterschiede verantwortlich, die in der Äquatorebene zwischen IKG und KMG herrschen. Im Verhältnis zum homogenen Modell a wird dieser Dichteunterschied in den Modellen $c$ und $f$ verkleinert. Dort ist der KMGWärmefluß in Äquatornähe nämlich unterdurchschnittlich klein. Dadurch ist die Stärke der Konvektion in diesen beiden Modellen geringer als im Referenzmodell a. Das läßt sich an der kinetischen Energie oder der magnetischen Reynoldszahl Rm (s. Tab. 4.2) ablesen, die bei konstantem $\eta$ ein Maß für die durchschnittliche Flüssigkeitsgeschwindigkeit in der Kugelschale ist (Seite 20). Sie beträgt in beiden Modellen etwa $\mathrm{Rm} \approx 475$, während sie in den Modellen d und e mit überdurchschnittlichem KMG-Wärmefluß am Äquator bei 560 bzw. 530 liegt. Außer den beiden nicht umkehrenden Modellen haben alle Modelle magnetische Reynoldszahlen $\mathrm{Rm}>500$. Die Modelle a, g und $\mathrm{H}$ sind sich in ihrer Umpolfrequenz sehr ähnlich und haben auch vergleichbare Rm zwischen 503 und 520. Das Modell b mit der höchsten Rm von 570 hat eine stark schwankende Dipolrichtung.

Die hier gemachten Beobachtungen weisen keine Diskrepanzen zu den Resultaten von G99 auf, bis auf die bemerkenswerte Ausnahme bei den Modellen e und $f$ mit einer $(\ell=4$, $m=0$ )-Wärmeflußvariation. Unser Modell e hat eine der höchsten Umpolfrequenzen, während das vergleichbare Modell bei G99 über 300000 Jahre in der gleichen Polarität verbleibt. Unser Modell $\mathrm{f}$ dagegen ist ein stabiler dipol-dominierter Dynamo, wogegen das Magnetfeld des vergleichbaren Modells bei G99 nach etwa 150000 Jahren zerfällt. Für die Wärmeflußmuster c und e beobachten G99 eine bzw. keine Umkehrung. Die geringe Anzahl an Umkehrungen dieser Modelle führen sie auf den hohen Wärmefluß in polaren Regionen zurück, welcher die polaren Aufströme verstärkt. Diese vergrößern durch den sie umgebenden thermischen Wind (s. Glatzmaier \& Roberts (1997)) die Scherströmungen im Tangentialzylinder, durch welche das achsenparallele Dipolfeld stabilisiert wird.

Die Frequenz von Polumkehrungen hängt also stark von der Wärmeflußverteilung an der Kern-Mantel-Grenze ab, und damit von der thermischen Struktur des untersten Erdmantels. Ob eine bestimmte Konfiguration des Wärmeflusses an der KMG aber zu einer Erhöhung oder Verringerung der Umpolfrequenz führt, ist zusätzlich von den Feld erzeugenden Mechanismen im Dynamo abhängig. In den Modellen von Glatzmaier et al. findet sich starkes toroidales Feld typischerweise nur innerhalb des Tangentialzylinders (Siehe Glatzmaier et al. (1999), dort Fig. 2 links unten). In den hier gerechneten Modellen ist starkes toroidales Feld auch außerhalb des Tangentialzylinders präsent, z. B. in den äußeren Regionen des Kerns nahe der Äquatorebene. Dieser grundlegende Unterschied könnte auf verschiedene Werte der physikalischen Parameter der Modelle zurückzuführen sein, und/oder auf die Tatsache, daß G99 Hyperdiffusivitäten verwenden. Bei G99 führen starke Flußvariationen zu einem im Vergleich mit der Erde zu niedrigen Dipolmoment. Der Schluß, daß die thermischen 
Inhomogenitäten über der KMG deshalb nicht groß sein können, läßt sich aus unseren Modellen nicht ableiten. Eine Veränderung des Dipolmomentes und der Umkehrfrequenz läßt sich auch bei vorgegebenem KMG-Wärmeflußmuster noch durch Variation der Rayleighzahl erreichen.

Für unsere Modelle ist entscheidend, ob der KMG-Wärmefluß innerhalb eines äquatorialen Gürtels hoch oder niedrig ist. Hoher äquatorialer Wärmefluß führt zu einer Erhöhung der Umpolfrequenz, während niedriger äquatorialer Wärmefluß die Umkehrungen ganz unterbinden kann. Eine Veränderung der thermischen Struktur des unteren Mantels in Äquatornähe führt zu einer Veränderung der Umkehrfrequenz. Das Ende des 40 Mio. Jahre andauernden Superchrons der Kreidezeit könnte z. B. durch die Ankunft einer abtauchenden tektonischen Platte an der KMG eingeleitet worden sein. 


\section{Zusammenfassung}

\section{Dynamoregimes und Polumkehrungen}

Mit der Entwicklung leistungsstarker Computer wurden in den 90er Jahren erstmals dreidimensionale numerische Dynamos simuliert, welche die wichtigsten im Erdkern geltenden physikalischen Gleichungen lösen. In einigen Veröffentlichungen wird auch von Polumkehrungen berichtet. Meist wurden Umkehrungen in Modellen beobachtet, die skalenabhängige Diffusivitäten benutzen, welche in Verdacht stehen, künstliche Polaritätswechsel auszulösen. Außerdem war nicht klar, warum in einige Modelle Umkehrungen aufweisen aber andere nicht.

Durch die hier durchgeführte Untersuchung werden erstmals die Regionen im Parameterraum aufgezeigt, in denen Feldumkehrungen auftreten können. Dadurch wurde gezeigt, daß das Auftreten und die Frequenz von Polumkehrungen zuallererst von der Wahl der fundamentalen Kontrollparameter abhängt, insbesondere der Rayleigh- und der Ekmanzahl. Das bedeutet $u$. a., daß Umkehrungen aus dem Dynamoprozeß als solchem entstehen und es auch keines von außen kommenden Auslösers bedarf, um eine Umkehrung einzuleiten.

Die Dynamo-Lösungen in verschiedenen Bereichen des Parameterraums können in zwei Regimes unterteilt werden, die sich durch die Art des erzeugten Magnetfeldes deutlich unterscheiden. Bei relativ kleinen Rayleighzahlen oberhalb des kritischen Wertes für den Einsatz selbsterhaltender Dynamowirkung werden die Magnetfelder von einem axialen Dipol stabiler Polarität dominiert und es gibt keine Umkehrungen des Feldes. Bei hohen Rayleighzahlen, also heftiger Konvektion, trägt der magnetische Dipol nur wenig zum Gesamtfeld bei. Er fluktuiert dabei stark in seiner Richtung, bevorzugt dabei im Mittel aber eine Ausrichtung entlang der Rotationsachse. Die Grenze zwischen diesen beiden Dynamoregimes verschiebt sich mit sinkender Ekmanzahl zu höheren überkritischen Rayleighzahlen.

Desweiteren hängt die Lage der Regimegrenze von der Antriebsart der Konvektion ab. Für den Erdkern sind viele verschiedene Szenarien modelliert worden, in denen die Konvektion auf unterschiedliche Weise angetrieben wird. Die Antriebsart ist im wesentlichen durch die Verteilung der Quellen und Senken des Auftriebs gegeben. Auftrieb entsteht überall dort, wo die Flüssigkeit weniger dicht ist als ihre Umgebung, sei es durch eine erhöhte Temperatur oder durch einen größeren Anteil einer leichten Komponente wie im Fall der chemischen Konvektion. Im einfachsten und oft benutzten Fall eines vorgegebenen Temperaturkontrastes zwischen innerer und äußerer Grenze des Erdkerns liegen die Quellen des Auftriebs an der inneren Grenze, denn dort ist die Flüssigkeit aufgrund der hohen Temperatur leichter. Die Senken des Auftriebs sind an der äußeren Grenze zu finden, wo die Temperatur niedrig ist.

Wird die Konvektion durch einem festen Temperaturkontrast hervorgerufen, ist die Regimegrenze klar zu erkennen, z. B. am mittleren Dipolbeitrag zum Gesamtfeld, welcher im 
umkehrenden Regime deutlich schwächer ist, als im stabilen. In den Fällen mit vorgeschriebenem Wärmefluß an einer oder beiden Rändern sowie im Falle chemisch angetriebener Konvektion sinkt der Dipolanteil eher allmählich mit steigender Rayleighzahl. Für die Modelle, deren Antrieb größtenteils durch interne Wärmequellen erfolgt (Quellen des Auftriebs im Volumen verteilt), wurde nur das Regime von Dynamos mit kleinen Dipolbeiträgen gefunden. Damit wird eine Brücke zu den Ergebnissen von Grote et al. (2000b) geschlagen, deren intern geheizte Dynamos bei Prandtlzahlen und Robertszahlen um 1.0 quadrupolare Feldgeometrien den dipolaren bevorzugen.

Im Übergangsbereich zwischen den beiden Regimes wurden Dynamos gefunden, welche dem Geodynamo in verschiedenen Aspekten ähnlich sind. Hier wechselt der Dynamo zwischen zwei Zuständen hin und her: mal wird das Magnetfeld von einem stabilen, axialen Dipol dominiert, mal ist der Dipol schwach und seine Richtung wechelt. Umkehrungen und Exkursionen treten dann auf, wenn der Dynamo in den Zustand schwachen Dipolfeldes wechselt und dann wieder, eventuell in anderer Polarität, den dipol-dominierten Zustand erreicht.

Ausgewählte Dynamos reproduzieren Eigenschaften des Geodynamos in erstaunlich guter Weise. Das durchschnittliche Dipolmoment ist dem mittleren Dipolmoment aus paläomagnetischen Untersuchungen vergleichbar, d. i. $(4-6) \times 10^{22} \mathrm{~A} / \mathrm{m}^{2}$, die Umkehrfrequenz liegt in der Größenordnung von einigen Umkehrungen pro Million Jahre. Auch der zeitliche Verlauf der Feldstärke während einer Umkehr ist mit den paläomagnetischen Beobachtungen vergleichbar: Vor der Umkehr sinkt die Feldstärke, dann finden die stärksten Richtungsänderungen der Dipolachse statt, worauf sich die Feldstärke wieder erholt. Während der Umkehrungen sinkt der Dipolbeitrag zum Oberflächenfeld ab, meist sogar sehr stark. In einem Fall ist das Obeflächenfeld während einer Umkehr dipol-dominiert geblieben. Wenn man als Dauer einer Polumkehr den Zeitraum bezeichnet, in dem die Dipolachse stark (z. B. > $30^{\circ}$ ) von der Rotationsachse abweicht, erhält man typischerweise Werte zwischen 1500 und 15000 Jahren für verschiedene Umkehrungen, in Übereinstimmung mit Abschätzungen geomagnetischer Umpolungen. Solch gute Übereinstimmung ist nur in einem sehr eingeschränkten Parameterbereich zu finden. Bei etwas zu kleinen Rayleighzahlen ist das durchschnittliche Dipolmoment größer als der paläomagnetische Mittelwert und Umkehrungen zu selten, bei etwas zu großen Rayleighzahlen verhält es sich genau umgekehrt. Alle heutigen numerischen Dynamo-Modelle überschätzen den Einfluß der viskosen Reibung enorm, d. h. sie nehmen zu niedrige Werte der Rayleigh-, und zu hohe Werte der Ekman- und Robertszahl an. Möglicherweise wird der Grenzbereich zwischen den Regimes, also der Bereich, in dem „,erdähnliche“ Polumkehrungen zu beobachten sind, wesentlich breiter, wenn realistischere Werte für die Viskosität angenommen werden können.

\section{Virtuelle Polverteilungen und thermische Kern-Mantel-Kopplung}

In verschiedenen paläomagnetischen Untersuchungen wurde von Häufungen der virtuellen geomagnetischen Pole (VGP's) berichtet. Je nach Selektion und Herkunft der Daten (ob Sedimente oder Laven) wurden diese Häufungen von einigen Autoren beobachtet aber von anderen Autoren nicht. Seit Anfang des letzten Jahrzehnts ist umstritten, ob diese Häufungen 
wirklich sind oder nur ein Artefakt. Da die VGP-Häufungen mit den Bereichen hoher seismischer Geschwindigkeit im unteren Erdmantel zusammenfallen, sind Temperaturunterschiede oberhalb der Kern-Mantel-Grenze als Ursache dieser Häufungen vorgeschlagen worden.

Mit der im ersten Teil dieser Arbeit durchgeführten Studie wurde herausgefunden, bei welchen Parametern Umkehrungen des Magnetfeldes auftreten. Es wurde auch gezeigt, in welchem Bereich diese Umkehrungen ,erdähnliche“ Eigenschaften aufweisen. Damit wurde die Voraussetzung geschaffen, zu testen, ob thermische Inhomogenitäten an der KernMantel-Grenze (KMG) tatsächlich zu beobachtbaren Häufungen der virtuellen Pole führen.

Für verschiedene Geometrien der Wärmeflußverteilung an der KMG wurden deswegen die VGP's berechnet und auf Häufungen analysiert. Desweiteren wurde der Einfluß von insgesamt acht verschiedenen Wärmeflußmustern auf die Umpolfrequenz eines Dynamos untersucht.

Eine longitudinale Variation entsprechend einer $(\ell=2, m=2)$-Kugelfunktionsmode führt dazu, daß sich die virtuellen Pole bei den Längen maximalen Wärmeflusses häufen. Die mittlere Häufung wird sichtbar, wenn die virtuellen Pole für mehrere Sites über einen ausreichend langen Zeitraum ( $\geq 2$ magnetische Diffusionszeiten oder etwa 300000 Jahre) betrachtet werden. Für kürzere Zeitspannen sind die VGP-Häufungen nicht notwendigerweise mit den Regionen hohen KMG-Wärmeflusses korelliert. Bei Exkursionen oder durch hohe Säkularvariation treten auch in Zeiten stabiler Polarität transiente VGP's auf, also solche, die in einem Abstand von mehr als $30^{\circ}$ von den Rotationspolen liegen. Auch die transienten VGP's stabiler Phasen häufen sich bei Längen maximalen KMG-Wärmeflusses.

Für die Modelle, deren KMG-Wärmeflußmuster aus der seismischen Tomographie abgeleitet ist, ergeben sich erhöhte mittlere VGP-Dichten bei geographischen Längen Ostasiens und der amerikanischen Kontinente. Im Modell liegen die Maxima der mittleren VGP-Dichte etwas westlich von den Maxima, die von Clement (1991) (Abb. 4.2) und Laj et al. (1991) (Abb. 4.1) für einzelne Umkehrungen gefunden wurden.

Als Mechanismus, der zu den Häufungen der virtuellen Pole führt, kommt folgender in Betracht: Variationen des Wärmeflusses an der Kern-Mantel-Grenze führen zu einer mittleren Strömung im flüssigen Erdkern unterhalb der KMG. Durch Advektion von Feldlinien entstehen im zeitgemittelten Magnetfeld Flußkonzentrationen in der Nähe der Stellen, an denen sich im mittleren Geschwindigkeitsfeld Abströme befinden (Olson \& Christensen, 2002). Die virtuellen Pole liegen bevorzugt in den Längen, in denen (im Mittel) die magnetischen Flußkonzentrationen zu finden sind (Gubbins \& Sarson, 1994; Gubbins \& Love, 1998).

Für ihr Modell mit einem aus der seismischen Tomographie abgeleiteten Wärmeflußmuster finden Olson \& Christensen (2002), daß diese mittleren magnetischen Flußkonzentrationen für kleinere Ekmanzahlen nach Osten wandern. Die Bereiche erhöhter VGP-Dichte würden somit für kleinere Ekmanzahlen auch etwas weiter nach Osten wandern, wodurch sie noch besser mit den Beobachtungen übereinstimmen würden.

Für das Modell mit der $(\ell=2, m=2)$-Geometrie des Wärmeflußmusters, welches dem tomographischen Modell ähnlich ist, wurden auch die Verteilungen der virtuellen Pole in den kurzen (einige Tausend Jahre langen) Zeitabschnitten von Umkehrungen betrachtet. In den meisten Fällen liegen die VGP's deutlich gehäuft, und zwar über jeweils einer der beiden Längen erhöhten Wärmeflusses. Eine Häufung in zwei Längenintervallen für eine einzelne Umkehr (wie für die Matuyama-Brunhes Umkehr gefunden, Abb. 4.2) konnte nur in einem einzigen Fall beobachtet werden, in dem die Umkehr allerdings überdurchschnittlich lange 
dauert (etwa 30000 Jahre). Die wesentlichen Merkmale der VGP-Verteilung einer einzelnen Umkehr werden schon mit den Daten von fünf einigermaßen gleichmäßig verteilten Sites wiedergegeben.

An der Erdoberfläche ist auch während der Umkehrungen die meiste Zeit ein signifikanter Dipolbeitrag zum Feld vorhanden. Die virtuellen Pole häufen sich in solchen Zeiten um die Position des wirklichen Pols (des TGP).

Neben der Rayleighzahl hat die Geometrie des Wärmeflußmusters an der KMG einen großen Einfluß auf das Umkehrverhalten eines Dynamos. Relativ zum homogenen Referenzmodell wirkt sich ein höherer Wärmefluß in äquatorialen Regionen wie eine Erhöhung der Rayleighzahl aus: Der Dipolbeitrag nimmt dadurch ab und Polumkehrungen werden wahrscheinlicher. Ein unterdurchschnittlicher Wärmefluß in äquatorialen Regionen führt zum umgekehrten Effekt, so daß der Dynamo eventuell gar nicht mehr umkehrt. Zusammen mit der Tatsache, daß die Zeitskala der Mantelkonvektion dieselbe ist wie die Zeitskala, auf der Änderungen der mittleren Umpolfrequenz auftreten, ist es ist sehr wahrscheinlich, daß die Umkehrfrequenz des Geodynamos von den thermischen Gegebenheiten um unteren Mantel kontrolliert wird.

Insgesamt konnte im Rahmen der Untersuchungen ein breites Spektrum an DynamoLösungen gefunden werden. Neben dipol-dominierten, quadrupolaren und hemisphärischen Dynamos wurden umpolende Dynamos gefunden, die in regelmäßiger, nahezu regelmäßiger und chaotischer Weise ihre Polarität wechseln und sich damit für weitere Studien z. B. des Umkehrmechanismus anbieten oder als Modell für den umpolenden Geodynamo dienen können. 


\section{Literaturverzeichnis}

Barton C.E.; McFadden P.L., 1996. Inclination shallowing and preferred transitional VGP paths. Earth Planet. Sci. Lett., 140, 147-157.

Batchelor G.K., 1967. An introduction to fluid dynamics. Cambridge University Press.

Bloxham J., 2000. Sensitivity of the geomagnetic axial dipole to thermal core-mantle interactions. Nature, 405, 63-65.

Bloxham J.; Jackson A., 1992. Time-dependent mapping of the magnetic field at the coremantle boundary. J. Geohys. Res., 97, 19537-19563.

Busse F.H., 1975. A model of the geodynamo. Geophys. J. Roy. Astron. Soc., 42, 437-459.

Christensen U.R.; Aubert J.; Cardin P.; Dormy E.; Gibbons S.; Glatzmaier G.A.; Grote E.; Honkura Y.; Jones C.; Kono M.; Matsushima M.; Sakuraba A.; Takahashi F.; Tilgner A.; Wicht J.; Zhang K., 2001. A numerical dynamo benchmark. Phys. Earth Planet. Int., 128, $25-34$.

Christensen U.R.; Olson P.; Glatzmaier G.A., 1998. A dynamo model interpretation of geomagnetic field structures. Geophys. Res. Lett., 25, 1565-1568.

Christensen U.R.; Olson P.; Glatzmaier G.A., 1999. Numerical modelling of the geodynamo: A systematic parameter study. Geophys. J. Int., 138, 393-409.

Clement B.M., 1991. Geographical distribution of transitional VGPs: Evidence for nonzonal equatorial symmetry during the Matuyama-Brunhes geomagnetic reversal. Earth Planet. Sci. Lett., 104, 48-58.

Clement B.M.; Kent D.V., 1991. A southern hemisphere record of the Matuyama-Brunhes polarity reversal. Geophys. Res. Lett., 18, 81-84.

Coe R.S.; Hongre L.; Glatzmaier G.A., 2000. An examination of simulated geomagnetic reversals from a palaeomagnetic perspective. Phil. Trans. R. Soc. Lond. A, 358, 11411170.

Dormy E.; Valet J.P.; Courtillot V., 2000. Numerical models of the geodynamo and observational constraints. Geochem. Geophys. Geosyst., 1. Artikel Nr. 2000 GC000062.

Dziewonski A.M.; Woodhouse J.H., 1987. Global images of the Earth's interior. Science, 236, 37-48. 
Fearn D.R., 1998. Hydromagnetic flow in planetary cores. Rep. Prog. Phys., 61, 175-235.

Glatzmaier G.; Roberts P.H., 1997. Simulating the geodynamo. Cont. Phys., 38, 269-288.

Glatzmaier G.A., 1984. Numerical simulations of stellar convective dynamos. I. the model and method. J. Comput. Phys., 55, 461-484.

Glatzmaier G.A., 2002. Geodynamo simulations-how realistic are they? Annu. Rev. Earth Planet. Sci., 30, 237-257.

Glatzmaier G.A.; Coe R.S.; Hongre L.; Roberts P.H., 1999. The role of the Earth's mantle in controlling the frequency of geomagnetic reversals. Nature, 401, 885-890.

Glatzmaier G.A.; Roberts P.H., 1995a. A 3D convective dynamo solution with rotating and finitely conducting inner core and mantle. Phys. Earth Planet. Int., 91, 63-75.

Glatzmaier G.A.; Roberts P.H., 1995b. A 3D self-consistent computer simulation of a geomagnetic field reversal. Nature, 377, 203-209.

Goguitchaichvili A.; Urrutia-Fucugauchi J.; Alva-Valdivia L., 2002. Mesozoic dipole low: Myth or reality? EOS, 83(41), 457-461. American Geophysical Union.

Grote E.; Busse F.H.; Tilgner A., 1999. Convection-driven quadrupolar dynamos in rotating spherical shells. Phys. Rev. E, 60, 5025-5028.

Grote E.; Busse F.H.; Tilgner A., 2000a. Effects of hyperdiffusivities on dynamo simulations. Geophys. Res. Lett., 27, 2001-2004.

Grote E.; Busse F.H.; Tilgner A., 2000b. Regular and chaotic spherical dynamos. Phys. Earth Planet. Int., 117, 259-272.

Gubbins D., 1999. The distinction between geomagnetic excursions and reversals. Geophys. J. Int., 137, F1-F3.

Gubbins D.; Love J.J., 1998. Preferred VGP paths during geomagnetic polarity reversals: Symmetry considerations. Geophys. Res. Lett., 25(7), 1079-1082.

Gubbins D.; Roberts P.H., 1987. Magnetohydrodynamics of the Earth's core, in: Geomagnetism Vol. 2, Editor J. A. Jacobs. Academic Press, London.

Gubbins D.; Sarson G., 1994. Geomagnetic field morphologies from a kinematic dynamo model. Nature, 368, 51-55.

Herrero-Bervera E.; Theyer F.; Helsley C.E., 1987. Olduvai onset polarity transition: two detailed palaeomagnetic records from North Central Pacific sediments. Phys. Earth Planet. Int., 49, 325ff.

Hoffman K.A., 2000. Temporal aspects of the last reversal of the Earth's magnetic field. Phil. Trans. R. Soc. Lond. A, 358, 1181-1190. 
Hollerbach R.; Jones C.A., 1993. Influence of the Earth's inner core on geomagnetic fluctuations and reversals. Nature, 365, 541-543.

Jackson A.; Jonkers A.R.T.; Walker M.R., 2000. Four centuries of geomagnetic secular variation from historical records. Phil. Trans. R. Soc. Lond., 358, 957-990.

Jacobs J.A., 1994. Reversals of the Earth's magnetic field. Cambridge University Press, second edition.

Jones C.A.; Longbottom A.W.; Hollerbach R., 1995. A self-consistent convection-driven geodynamo model, using a mean-field approximation. Phys. Earth Planet. Inter., 92, 119141.

Kageyama A.; Ochi M.; Sato T., 1999. Flip-flop transitions of the magnetic intensity and polarity reversals in the MHD dynamo. Phys. Rev. Lett., 82, 5409-5412.

Kageyama A.; Sato T., 1997a. Dipole field generation by a MHD dynamo. Plasma Phys. Control. Fusion, 39, A83-A91.

Kageyama A.; Sato T., 1997b. Generation mechanism of a dipole field by a MHD dynamo. Phys. Rev. E, 55, 4617-4626.

Kageyama A.; Sato T., 1997c. Velocity and magnetic field structures in MHD dynamo. Phys. Plasma, 4(5), 1569-1575.

Katayama J.; Matsushima M.; Honkura Y., 1999. Some characteristics of magnetic field behavior in a model of MHD dynamo thermally driven in a rotating spherical shell. Phys. Earth Planet. Int., 111, 141-159.

Kida S.; Araki K.; Kitauchi H., 1997. Periodic reversals of magnetic field generated by thermal convection in a rotating spherical shell. J. Phys. Soc. Jap., 66, 2194-2201.

Kida S.; Kitauchi H., 1998. Thermally driven MHD dynamo in a rotating spherical shell. Prog. Theoret. Phys. Suppl., 130, 121-136.

Kitauchi H.; Kida S., 1998. Intensification of magnetic field by concentrate-and-stretch of magnetic flux lines. Phys. Fluids, 10(2), 457-468.

Kono M.; Roberts P.H., 2002. Recent geodynamo simulations and observations of the geomagnetic field. Rev. Geoph., AGU. Artikel Nr. 10.1029/2000RG000102.

Kuang W.; Bloxham J., 1997. An Earth-like numerical dynamo model. Nature, 389, 371374.

Kuang W.; Bloxham J., 1998. Numerical dynamo modeling: Comparison with the Earth's magnetic field. In: The Core-Mantle Boundary Region, Geodyn. Ser., 28, 197-208. Herausgeber: M. Gurnis, M.E. Wysession, E. Knittle, B.A. Buffett, AGU, Washington DC.

Kutzner C., 1999. Numerische Simulationen des Geodynamos: Einfluß verschiedener Antriebsarten auf Strömung und Magnetfeld. Diplomarbeit, Institut für Geophysik der Universität Göttingen. 
Kutzner C.; Christensen U.R., 2000. Effects of driving mechanisms in geodynamo models. Geophys. Res. Lett., 27(1), 29-32.

Laj C.; Mazaud A.; Weeks R.; Fuller M.; Herrero-Bervera E., 1991. Geomagnetic reversal paths. Nature, 351, 447.

Langel R.A.; Estes R.H., 1982. A geomagnetic field spectrum. Geophys. Res. Lett., 9, 250-253.

Langereis C.G.; Hoof A.A.M.V.; Rochette P., 1992. Longitudinal confinement of geomagnetic reversal paths as a possible sedimentary artefact. Nature, 358, 226-230.

Lister J.R.; Buffett B.A., 1995. The strength and efficiency of thermal and compositional convection in the geodynamo. Phys. Earth. Planet. Int., 91, 17-30.

Loper D.E.; Roberts P.H., 1981. A study of conditions at the inner core boundary of the Earth. Phys. Earth Planet. Inter., 24, 302-307.

Love J.J., 1998. Paleomagnetic volcanic data and geometric regularity of reversals and excursions. J. Geophys. Res., 103, 12435-12452.

Love J.J.; Mazaud A., 1997. A database for the Matuyama-Brunhes magnetic reversal. Phys. Earth Planet. Int., 103, 207-245.

Masters G.; Johnson S.; Laske G.; Bolton H., 1996. A shear-velocity model of the mantle. Phil. Trans. R. Soc. Lond. A, 354, 1385-1411.

Merrill R.T.; McFadden P.L., 1999. Geomagnetic polarity transitions. Rev. Geophys., 37, 201-226.

Olejniczak R., 2002. Numerische Simulationen des Geodynamos: Einfluß von Trägheitseffekten auf Strömung und Magnetfeld. Diplomarbeit, Institut für Geophysik der Universität Göttingen.

Olson P., 2002. The disappearing dipole. Nature, 416, 591-594.

Olson P.; Christensen U.R., 2002. The time-averaged magnetic field in numerical dynamos with non-uniform boundary heat flow. Geophys. J. Int., 151, 809-823.

Olson P.; Christensen U.R.; Glatzmaier G.A., 1999. Numerical modeling of the geodynamo: Mechanisms of field generation and equilibration. J. Geophys. Res., 104, 10383-10404.

Olson P.; Glatzmaier G.A., 1995. Magnetoconvection in a rotating spherical shell: structure of flow in the outer core. Phys. Earth Planet. Int., 92, 109-118.

Poirer J.P., 1994. Light elements in the Earth's outer core: A critical review. Phys. Earth Planet. Inter., 85, 319-337.

Prévot M.; Camps P., 1993. Absence of preferred longitude sectors for poles from volcanic records of geomagnetic reversals. Nature, 366, 53-57. 
Sakuraba A.; Kono M., 1999. Effect of the inner core on the numerical simulation of the MHD dynamo. Phys. Earth Planet. Int., 111, 105-121.

Sarson G.; Jones C., 1999. A convection driven geodynamo reversal model. Phys. Earth Planet. Int., 111, 3-20.

Singer B.S.; Pringle M.S., 1996. The age and duration of the Matuyama-Brunhes geomagnetic polarity reversal from ${ }^{40} \mathrm{Ar} /{ }^{39} \mathrm{Ar}$ incremental heating analyses of lavas. Earth Planet. Sci. Lett., 139, 47-61.

Su W.J.; Woodward R.L.; Dziewonski A.N., 1994. Degree-12 model of shear velocity heterogeneity in the mantle. J. Geophys. Res., 99, 6945-6980.

Tauxe L., 1998. Paleomagnetic principles and practice, in: Modern approaches in geophysics, Vol. 17. Kluwer Academic Publishers, Dordrecht, Niederlande.

Tric E.; Laj C.; Jéhanno C.; Valet J.P.; Kissel C.; Mazaud A.; Iaccarino S., 1991. Highresolution record of the Upper Olduvai transition from Po Valley (Italy) sediments: support for dipolar transition geometry? Phys. Earth Planet. Int., 65, 319-336.

Valet J.P.; Tucholka P.; Courtillot V.; Meynadier L., 1992. Paleomagnetic constraints on the geometry of the geomagnetic field during reversals. Nature, 356, 400-407.

van der Hilst R.D.; Widiyantoro S.; Creager K.C.; McSweeney T.J., 1998. Deep subduction and aspherical variations in P-wavespeed at the base of the Earth's mantle. In: The coremantle boundary region, Geodynamics series, 28, 5-20.

Weinstein S.; Olson P., 1990. Planforms in thermal convection with internal heat sources at large Rayleigh and Prandtl numbers. Geophys. Res. Lett., 17, 239-242.

Wicht J., 2002. Inner-core conductivity in numerical dynamo simulations. Phys. Earth Planet. Int., 132, 281-302.

Wysession M.E.; Lay T.; Revenaugh J.; Williams Q.; Garnero E.J.; Jeanloz R.; Kellogg L.H., 1998. The D" discontinuity and its implications. In: The core-mantle boundary region, Geodynamics series, 28, 273-297.

Zhang K.; Jones C., 1997. The effect of hyperviscosity on geodynamo models. Geophys. Res. Lett., 24, 2869-2872. 


\section{Danksagung}

An erster Stelle möchte ich Prof. Ulrich R. Christensen danken, der mich zu diesem interessanten und sehr ergiebigen Thema motiviert hat. Ich weiß es sehr zu schätzen, daß er es mir ermöglicht hat, an zahlreichen nationalen und internationalen Tagungen teilzunehmen und dort meine Ergebnisse zu präsentieren.

Ich danke Dr. Dieter Schmitt vom Max-Planck-Institut für Aeronomie für die bereitwillige Übernahme des Korreferats.

Mein besonderer Dank gilt meinen Eltern Ursula und Frank Kutzner, die mich während meiner gesamten Studienlaufbahn finanziell, moralisch und organisatorisch unterstützt haben. Danke!! Ohne Euch wäre das alles nicht machbar gewesen!

Vielen, vielen Dank an Johannes Wicht, der seine Zeit opferte, um die Rohfassung dieser Arbeit zu lesen. Er hat durch konstruktive Kritik sehr zum Gelingen beigetragen.

Ein großes Dankeschön geht an die Deutsche Forschungsgemeinschaft, die dieses Projekt durch finanzielle Unterstützung ermöglichte.

Ich möchte mich bei Ulrich Einecke für die unverzichtbare Betreuung des Computernetzes bedanken. Rainer Hennings danke ich für diverse Paper, welche er mir aus den Untiefen verschiedener Bibliotheken herzauberte.

Alexander - danke für ungezählte Tips zum Thema $\mathrm{LTT}_{\mathrm{E}} \mathrm{X}$ und dazu, was bei einer Disputation alles organisiert werden will!

Ich möchte herzlichst dem „Team Altbau II. Stock“ danken: Julien, Monika, und Denise, die immer hart arbeiten, aber zwischendurch auch Zeit für eine Tasse Kaffee finden. Last but not least bedanke ich mich bei allen Leuten am Institut für Geophysik in Göttingen. Es war eine schöne Zeit, die ich sicher sehr vermissen werde!!! 


\section{Lebenslauf}

Carsten Kutzner, geboren am 28. Juni 1972 in Göttingen, ledig, 1 Kind, Staatsangehörigkeit: deutsch

\section{Schulbildung}

1978 - 1982 Grundschule Bovenden

1982 - 1984 Orientierungsstufe Bovenden

1984 - 1991 Theodor-Heuss-Gymnasium Göttingen, Abschluß: Abitur

1990 - 1997 Leistung des Ersatzdienstes im Technischen Hilfswerk, Ortsverband Göttingen

\section{Studium}

ab 10 / 1991 Grundstudium der Physik an der Georg-August Universität, Göttingen

10 / 1993 Vordiplomprüfung Physik

ab 10 / 1993 Hauptstudium der Physik an der Georg-August Universität, Schwerpunkt Geophysik

11 / 1997 - 12 / 1997 Mitarbei im Eifel-Plume-Projekt als Studentische Hilfskraft am Institut für Geophysik, Göttingen

07 / 1998 - 09 / 1999 Studentische Hilfskraft am Institut für Geophysik, Mitarbeit bei der Betreuung des Unix-Clusters

11 / 1997 - 10 / 1999 Anfertigung der Diplomarbeit in der Arbeitsgruppe von Prof. U. R. Christensen am Institut für Geophysik. Thema der Arbeit: „Numerische Simulationen des Geodynamos: Einfluß verschiedener Antriebsarten auf Strömung und Magnetfeld"

10 / 1999 Diplomprüfung Physik

11 / 1999 - 03 / 2000 Wissenschaftlicher Mitarbeiter am Institut für Geophysik

03 / 2000 - 03 / 2003 Wissenschaftlicher Mitarbeiter am Institut für Geophysik, gefördert im Rahmen des Schwerpunktprogramms Erdmagnetische Variationen der Deutschen Forschungsgemeinschaft. Promotion in der Arbeitsgruppe von Prof. U. R. Christensen. Thema der Arbeit: „Untersuchung von Feldumkehrungen an einem numerischen Modell des Geodynamos"

$03 / 2002$ Vortragsauszeichnung durch die Deutsche Geophysikalische Gesellschaft anläßlich ihrer 62. Jahrestagung in Hannover. Titel des Vortrags „Polumkehrungen in einem erdähnlichen Dynamo-Modell“" 